\title{
NASA-CR 166,084
}

NASA Contractor Report 166084

NASA-CR-166084

19830022136

\section{Longitudinal Flying Qualities Criteria for Single-Pilot Instrument Flight Operations}

\author{
Aharon Bar-Gill \\ Flight Research Laboratory \\ Princeton University \\ Princeton, N.J. 08544 \\ Contract NAS1-15764 \\ July 1983
}

\section{N/Sก}

National Aeronautics and Space Administration

Langley Research Center Hampton, Virginia 23665 
NASA Contractor Report 166084

\section{Longitudinal Flying Qualities Criteria for Single-Pilot Instrument Flight Operations}

Aharon Bar-Gill

Flighe Research Laboratory

Princeton University

Princeton, N.J. 08544

Contract NAS1-15764

July 1983

\section{N/SA}

National Aeronautics and

Space Administration

Langley Research Center

Hampton, Virginia 23665 


\section{ABSTRACT}

This research used modern estimation and control theory, flight testing, and statistical analysis to deduce flying qualities criteria for General Aviation (GA). Single-Pilot Instrument Flight Rule (SPIFR) operations. The principal concern is that unsatisfactory aircraft dynamic response combined with high navigation/communication workload can produce problems of safety and efficiency. To alleviate these problems, one has to determine first the relative importance of these factors. This objective was achieved by flying SPIFR tasks with different aircraft dynamic configurations and assessing the effects of such variations under these conditions.

The experimental results yielded quantitative indicators of pilot's performance and workload, and for each of them, multivariate regression was applied to evaluate several candidate flying qualities criteria. Aircraft configurations that are representative of the low-frequency dynamic and trim-related airframe response, and whose ranges of variation cover both the existing light aircraft fleet and recent trends in GA design, were tested. The implicit model following algorithm was used to simulate these configurations 
using Princeton's Avionics Research Aircraft (ARA) and an onboard microprocessor-based digital data acquisition system.

The following important results were derived from this research:

* A phugoid mode that is too lightly damped, produces unsatisfactory flying qualities, and it may have an adverse effect on safety in the SPIFR flight regime. It is suggested that the phugoid damping ratio should not be lower than 0.04 , which is consistent with the criterion of the military flying qualities specifications.

* The current FAA reference to stick force sensitivity, $\Delta f_{s}^{*} / \Delta v_{\text {comm' is qualitative. Any quantification of this }}$ criterion is important, because it is equivalent to a perception by the pilot of having direct control of velocity, which simplifies the piloting task. Such a quantitative upper bound is indicated by the results of this research: $\Delta \mathrm{f}_{\mathrm{s}}^{*} / \Delta \mathrm{v}_{\mathrm{comm}} \leqslant 1.4 \mathrm{lb} / \mathrm{kt}$.

* The pitch attitude sensitivity to airspeed change from one trimmed flight condition to another, $\Delta \theta^{*} / \Delta \mathrm{v}_{\text {comm }}$ is a new criterion, suggested for judgement of the SPIFR . flying qualities. Its value should not be lower than $-0.7 \mathrm{deg} / \mathrm{kt}$. 
Objective performance metrics, such as tracking error RMS values, were found to agree with a single subject's opinion in SPIFR task evaluation. Also, the current research suggests that the Cooper-Harper pilot opinion scale is a good indicator of perceived pilot workload. Its results indicate that the SPIFR navigation/communication workload is important, compared to workload due to low-frequency aircraft dynamics.

In the course of this research a numerically robust algorithm for optimal flight path reconstruction was developed to assure accurate computation of the performance indicators. This algorithm uses aerodynamic and inertial measurements and the output of a microprocessor-based multiple Distance Measuring Equipment (DME) scanner as inputs, and it provides results in True North-East navigational coordinates. The position accuracy achieved via this integrated flight testing and trajectory estimation methodology, using low-cost instrumentation, was demonstrated in flight and may be readily applied to flight technical error evaluation of other navigational systems.

The main contributions of this research are the identification of longitudinal flying qualities criteria for the GA SPIFR flight regime, and the development of an integrated methodology for investigation of a complete flight regime, including accurate flight path reconstruction. 
This Page Intentionally Left Blank 
CONTENTS

ABSTRACT . . . . . . . . . . . . . . .

LIST OF TABLES . . . . . . . . . . . . . . . vi

LIST OF FIGURES . . . . . . . . . . . . . . v viii

NOMENCLATURE . . . . . . . . . . . . . . xi

Chapter

I. INTRODUCTION . . . . . . . . . . . . . . I-1

1.1 BACKGROUND . . . . . . . . . . . . . . . 1-1

1.2 GOALS AND SIGNIFICANT RESULTS ...... . $1-5$

1.3 ORGANIZATION OF THE REPORT . . . . . . . . 1-8

2. DERIVATION OF CANDIDATE FLYING QUALITIES CRITERIA AND

SPIFR EXPERIMENTAL DESIGN . . . . . . . . 2-1

2.1 AIRCRAFT DYNAMIC MODEL . . . . . . . . . 2-1

2.2 IDENTIFICATION OF SPIFR AERODYNAMIC CONFIGURATIONS AND CANDIDATE FLYING QUALITIES CRITERIA . . . . . . . . . . . 2-12

2.3 IMPLEMENTATION OF SPIFR CONFIGURATIONS VIA IMPLICIT-MODEL-FOLLOWING ALGORITHM . . . . 2-24

2.4 EFFECTS OF NAVIGATIONAL ACCURACY AND OF THE "LEARNING CURVE" ON MISSION PLANNING . . 2-30

2.5 IN-FLIGHT VERIFICATION OF THE SPIFR RESEARCH CONCEPT . . . . . . . . . . . . 2-35

3. FLIGHT PATH RECONSTRUCTION - THEORY AND APPLICATION 3-1

3.1 DYNAMIC MODEL GENERALIZATION . . . . . . . 3-1

3.2 THE OPTIMAL FILTERING AND SMOOTHING ALGORITHMS . . . . . . . . . . . . 3-10

3.3 FILTER TUNING AND IMPLEMENTATION OF THE OPTIMAL FLIGHT PATH RECONSTRUCTION ALGORITHM . . . . . . . . . . . 3-26

4. STATISTICAL ANALYSIS AND DERIVATION OF FLYING QUALITIES CRITERIA FOR SPIFR OPERATIONS . . . 4-1

4.1 THE MULTIPLE REGRESSION ALGORITHM . . . . . 4-3 4.2 THE FLIGHT-TEST-BASED PERFORMANCE INDICATORS 4-9 
4. 3 DERIVATION OF SPIFR FLYING QUALITIES

CRITERIA . . . . . . . . . . . . 4-30

5. SUMMARY, CONCLUSIONS AND RECOMMENDATIONS . . . . . 5-1

5. 1 SUMMARY OF THE RESEARCH METHODOLOGY . . . . 5-1

5.2 CONCLUSIONS AND RECOMMENDATIONS . . . . . 5-3

Appendix

page

A. AIRCRAFT AND DATA ACQUISITION SYSTEM PREPARATION • A-1

A.1 AIRCRAFT SYSTEM MODIFICATIONS . . . . . . . A-1

A. 2 INSTRUMENTATION AND DATA RECORDING SYSTEM • A-7

A. 3 SOFTWARE DEVELOPMENT . . . . . . . . . A-11

B. DERIVATION OF SPIFR CANDIDATE CRITERIA • • • • • • B-1

C. LINEARIZATION OF THE SPIFR MATHEMATICAL MODEL • • • C-1

C. 1 LINEARIZATION OF MODEL A . . . . . . . . $C-1$

C. 2 LINEARIZATION OF MODEL B . . . . . . . . . . C-2

C. 3 IMPROVEMENT OF THE ACCURACY OF DME/DME-BASED POSITION ESTIMATION VIA A DME/DME/DME

SCHEME . . . . . . . . . . . . . . C-9

D. POST-FLIGHT PROCESSING - DETAILS . . . . . . . . . D-1

D. 1 PREPROCESSING . . . . . . . . . . . . . . . D-1

D. 2 OPTIMAL FLIGHT PATH RECONSTRUCTION - INPUTS D-3

D. 3 OPTIMAL FLIGHT PATH RECONSTRUCTION - PROGRAM

FLOW CHARTS . . . . . . . . . . . . D D

E. PILOT COMMENTS AND DATA SUMMARY . . . . . . . . . . E-I

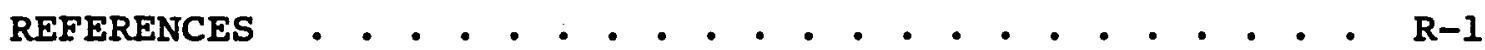




\section{LIST OF TABLES}

Table

page

2.1 Reference Number Assignment to the SPIFR Configurations . . . . . . . . . . . 2-21

2. 2 SPIFR Candidate Criteria . . . . . . . . . 2-22

2.3 Gain Settings for In-Flight simulation of SPIFR Configurations . . . . . . . . . . . 2-29

2.4 Experimental Verification of the Theoretical Dynamic Model . . . . . . . . . . . 2-39

3.1 Filter Tuning Results for Model A. . . . . . 3-34

3.2 Filter Tuning Results for Model B . . . . . . 3-40

4.1 PORs, Reactions-to-Workload-Lights and Adjustments of Throttle-Plus-Elevator Trim as Candidate Performance Indicators . . . . . . . . 4-16

4. 2 Longitudinal Stick Deflections RMS Values as Candidate Performance Indicators . . . . . 4-19

4.3 MLS and VOR Tracking RMS Values and Out-of-Band Time Percentages as candidate Performance Indicators . . . . . . . . . . . . 4-24

4.4 Altitude and Altitude Gradient Tracking RMS Values as Candidate Performance Indicators . . . . 4-28

4.5 Indicated AirSpeed Tracking RMS Values as Candidate Performance Indicators . . . . . . . . . 4-29

4.6 Computation of Flight Path Stability Parameter for SPIFR Configurations . . . . . . . . . 4-37

A.1 Input Assignments for SPIFR Digital Data Recording System • • • • • • • • • • • • • • • A-9

A.2 Output Assignments for SPIFR Digital Data Recording System . . . . . . . . . . . . . . A-10 
B.I Natural Response Characteristics of the SPIFR Configurations... . . . . . . . . B-2

B.2 Output Command Algorithm Application to SPIFR Configurations . . . . . . . . . . . . B-4

B.3 Airspeed and Flight Path Angle Rise Time Values - B-8

B.4 Airspeed and Flight Path Angle Overshoot Values . B-9

D. I Hexidecimal Code Recorded on the Channel that Provides Stations Switching Timings . . . . D-3

D. 2 Navigational Stations and Forrestal Airport Reference Point Coordinates . . . . . . . D-5 


\section{LIST OF FIGURES}

Figure

page

2.1 Total Damping Variations for SPIFR Configurations . . . . . . . . . . 2-23

2. 2 Block Diagram for Implicit Model-Following on the ARA . . . . . . . . . . . . . . . $2-25$

2.3 Implementation of a SPIFR Configuration on the InFlight Simulator . . . . . . . . . . 2-26

2.4 SPIFR Flight Path, Variant 1... . . . . . 2-32

2.5 SPIFR Flight Path, Variant 2. . . . . . . 2-32

2.6 SPIFR Flight Path, Variant 3... . . . . . 2-32

2.7 SPIFR Flight Path, Variant 4....... . . 2-32

2.8 Ground Stations Engagement in the VOR/VOR or the DME/DME Modes. . . . . . . . . . . 2-33

3.1. Earth Axes . . . . . . . . . . . . 3-5

3.2 Longitudinal Flight Path Segment . . . . . . 3-30

3.3 Coordinated Climbing Turn . . . . . . . . 3-30

3.4 Filter Tuning for Wind Estimation . . . . . . 3-42

3.5 Application of the Optimal Flight Path

Reconstruction Algorithm to the Climbing Turn

Generic Data ... . . . . . . . . 3-45

3.6 Application of the Optimal Flight Path Reconstruction Algorithm to Actual Flight-Test

Data . . . . . . . . . . . . 3-52

3.7 Starting Point Dispersion for the First Eleven SPIFR Tasks . . . . . . . . . . . . 3-59

3.8 Aircraft-Navigational Stations Geometry for the Starting Point for the First Eleven SPIFR Missions 
3.9 Reconstruction of a Navigational Trajectory Second

Variant . . . . . . . . . . . . 3-62

3.10 Reconstruction of a Navigational Trajectory Third

Variant... . . . . . . . . . 3-63

3.11 Reconstruction of a Navigational Trajectory Fourth

Variant . . . . . . . . . . . 3-64

3.12. Reconstruction of Airspeed and Altitude Profiles

for a Complete SPIFR Task . . . . . . . 3-65

4.1 SPIFR Research Flow Chart . . . . . . . . . 4-2

4.2 Knee-pad Versions of the Performance and Workload PORs and of the Evaluation Sheet . . . . . 4-11

4.3 Time Histories of the Longitudinal Stick, the Throttle and the Longitudinal Trim Wheel . 4-17

4.4 Localizer and Glide Slope Deviations . . . . . 4-21

4.5 VOR Deviations . . . . . . . . . . . . 4-22

4.6 Localizer Tracking RMS Error as a Function of

Pitch Attitude Sensitivity to Changes in

Airspeed . . . . . . . . . . . . 4 4-34

4.7 Rate-of-Descent Holding RMS Error Versus the

Phugoid Total damping . . . . . . . . 4-35

4.8 Flight Path Angle Versus Airspeed on Descent . 4-38.

A.l Avionics Research Aircraft, Navion N5ll3K . . A-3

A.2 Overview of the ARA In-Flight simulator system . A-4

A.3 Cockpit Displays of the Avionics Research

Aircraft. Modular SPIFR Evaluation Pilot Panel

at Left . . . . . . . . . . . . . A-6

A.4 SPIFR Digital Data Recording System . . . . . . A-8

B.1 Total Damping Histogram . . . . . . . . . . B-2

B.2 Change in Pitch attitude for lo-kt Airspeed
Retrimming . . . . . . . . . . B-5

B.3 Elevator Adjustment for 10-kt Airspeed Retrimming B-6

B.4 Elevator Adjustment for 4-deg Flight Path Angle

Retrimming . . . . . . . . . . . B-7 
B.5 Airspeed Rise Time for 1-deg Elevator Step Input B-10

B.6 Flight Path Angle Rise Time for 1-deg Elevator Step Input . . . . . . . . . . . . . B-10

B.7 Airspeed Rise Time for 108 Throttle Step Input . B-II

B.8 Flight Path Angle Rise Time for $10 \%$ Throttle Step Input . . . . . . . . . . . . . . . B-11

B.9 Airspeed Overshoot for 1-deg Elevator Step Input B-12

B.10 Flight Path Angle Overshoot for 1-deg Elevator Step Input . . . . . . . . . . . . B-12

B.Il Airspeed Overshoot for 108 Throttle Step Input . B-13

B.12 Flight Path Angle Overshoot for 108 Throttle Step Input . . . . . . . . . . . . . . B-13

C.1 Case Study of DME/DME/DME Position Estimation Accuracy . . . . . . . . . . . . c-10

D.1 Data Reduction Procedure . . . . . . . . . D-2

D.2 Forward Filtering Program . . . . . . . . . D-6

D. 3 Backward Smoothing Program . . . . . . . . . D-7 
This Page Intentionally Left Blank 


\section{NOMENCLATURE}

Variables

A

$A_{B}, A_{F}$

$A_{k}$

AR

a

$\underline{a}_{B}$

$a_{x} \cdot a_{y} \cdot a_{z}$

$\underline{\mathrm{b}}$

$\mathrm{b}_{\psi}$

$\mathrm{b}_{\mathrm{si}}$

C

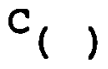

$c_{i j}$ overshoot absolute value

engineering correction matrices for the implicit model following scheme

smoother gain matrix

wing aspect ratio

semi-major-axis of the equator ellipse, ft

acceleration vector in body axes, "g"

cartesian components of $\underline{a}_{B}$ " " $g$ "

vector of expected values of

the regression coefficients

bias-type state in model A, deg

bias-type states in model $B$, ft

implicit model following gain matrix

nondimensional aerodynamic derivative

correction terms in the $F_{B}$ matrix

auxiliary definitions for

model linearization

$-$

c reference length, ft

D drag force along the flight

path, Ib

$\mathbf{E}$ statistical expectation operator 


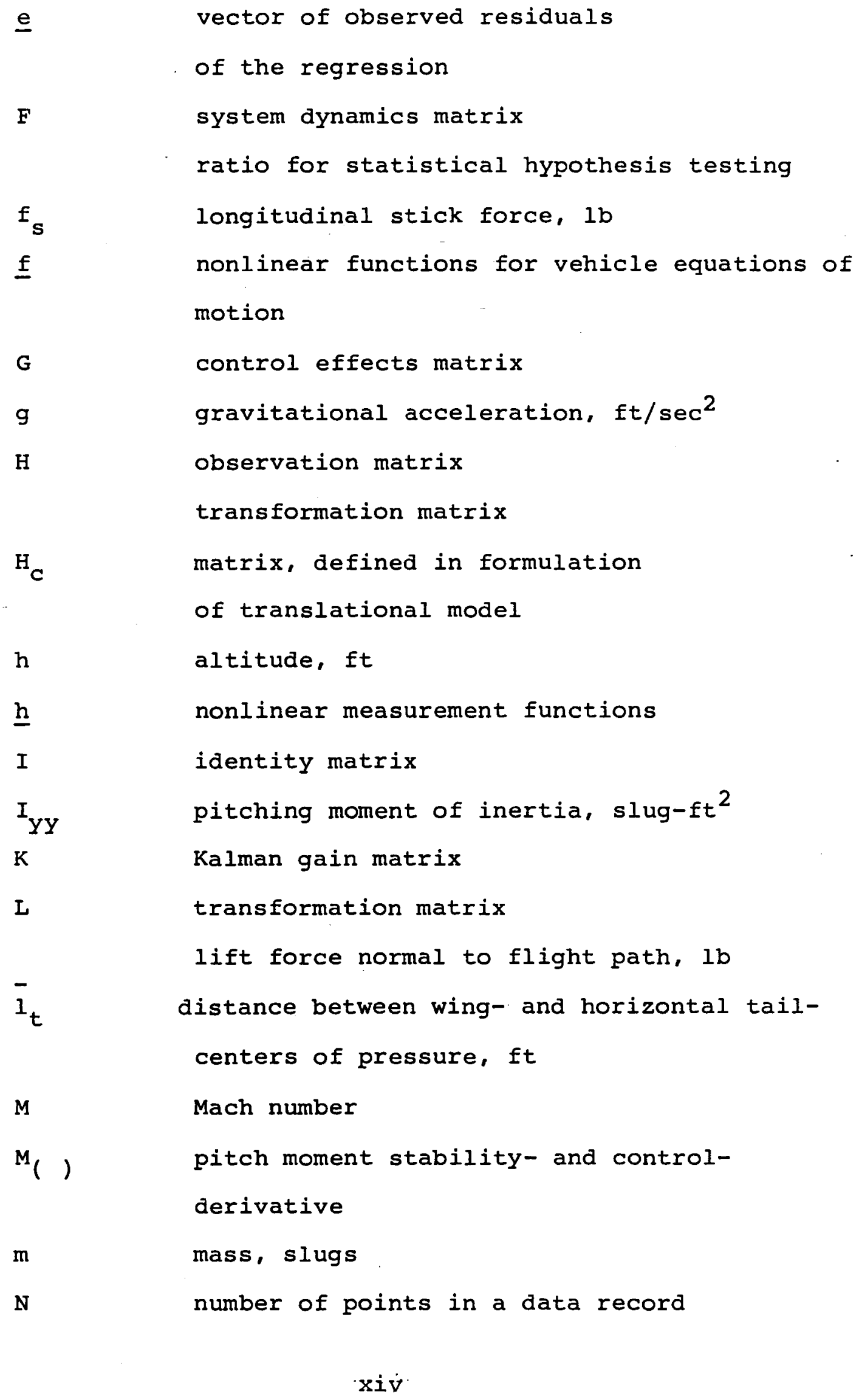


$n$

$n()$

P

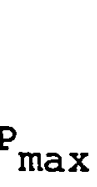

PI

$p$

$Q_{\mathbf{k}}$

$q$

$-$

$q$

R

$R_{\mathrm{k}}$

$\mathrm{R}^{2}$

$r$

$r_{\text {si }}$ length of vectors for regression random noise associated with the ( )variable

state covariance matrix

period of sinusoidal oscillation, sec maximum power available, lb

performance index

roll rate, deg/sec

number of candidate criteria vectors

process noise covariance matrix

pitch rate, deg/sec

dynamic pressure, $1 \mathrm{~b} / \mathrm{ft}^{2}$

aircraft distance from origin of Earthcentered coordinate frame, ft

measurement noise covariance matrix

multiple correlation coefficient

yaw rate, deg/sec

distance from the aircraft to the si-th

ground station, ft

$\mathrm{rx} 1, \mathrm{r} \times 2, \mathrm{rx} 3$ defined in eq. (C-61) to $(\mathrm{C}-63)$

$S$ intermediate command output matrix wing (reference) area, $\mathrm{ft}^{2}$

SSE(FM) sum of squares due to error associated with regression of the full model

(hypothesis testing) 


\begin{tabular}{|c|c|}
\hline $\operatorname{SSE}(\mathrm{RM})$ & $\begin{array}{l}\text { sum of squares due to error associated } \\
\text { with regression of the reduced model } \\
\text { (hypothesis testing) }\end{array}$ \\
\hline $\mathbf{s}$ & Laplace transform variable \\
\hline $\mathbf{T}$ & $\begin{array}{l}\text { out-of-band dwelling time, } 8 \\
\text { thrust, lb } \\
\text { duration of flight segment to be reconstruct- } \\
\text { ed, sec }\end{array}$ \\
\hline $\mathrm{TD}()$ & $\begin{array}{l}\text { (thrust+drag)-force-along-flight-path } \\
\text { derivative }\end{array}$ \\
\hline$T L()$ & $\begin{array}{l}\text { (thrust+lift)-force-normal-to-flight-path } \\
\text { derivative }\end{array}$ \\
\hline $\begin{array}{l}\mathrm{T}_{\mathrm{C}}^{\prime} \\
\mathrm{T}_{\max }\end{array}$ & $\begin{array}{l}\text { thrust coefficient or thrust force/qs } \\
\text { maximum thrust available for a given power } \\
\text { setting, lb }\end{array}$ \\
\hline$t$ & time, sec \\
\hline$t_{1 / 2}$ & $\begin{array}{l}\text { time to half amplitude of damped } \\
\text { sinusoidal oscillation, sec }\end{array}$ \\
\hline$\Delta t$ & time interval, sec \\
\hline $\mathrm{u}$ & $x$-axis velocity, $f t / s e c$ \\
\hline$\underline{\mathbf{u}}$ & $\begin{array}{l}\text { control vector } \\
\text { regression error vector }\end{array}$ \\
\hline$\underline{v}_{\text {air }}$ & airspeed vector, $\mathrm{ft} / \mathrm{sec}$ \\
\hline Var & $\begin{array}{l}\underline{\mathrm{V}}_{\text {air }} \mathrm{l} \text {, absolute value of } \mathrm{v}_{\mathrm{air}} \text {, ft/sec } \\
\text { statistical variance operator }\end{array}$ \\
\hline $\mathrm{v}$ & $\begin{array}{l}\mathrm{y} \text {-axis velocity, } \mathrm{ft} / \mathrm{sec} \\
\text { measurement noise }\end{array}$ \\
\hline
\end{tabular}




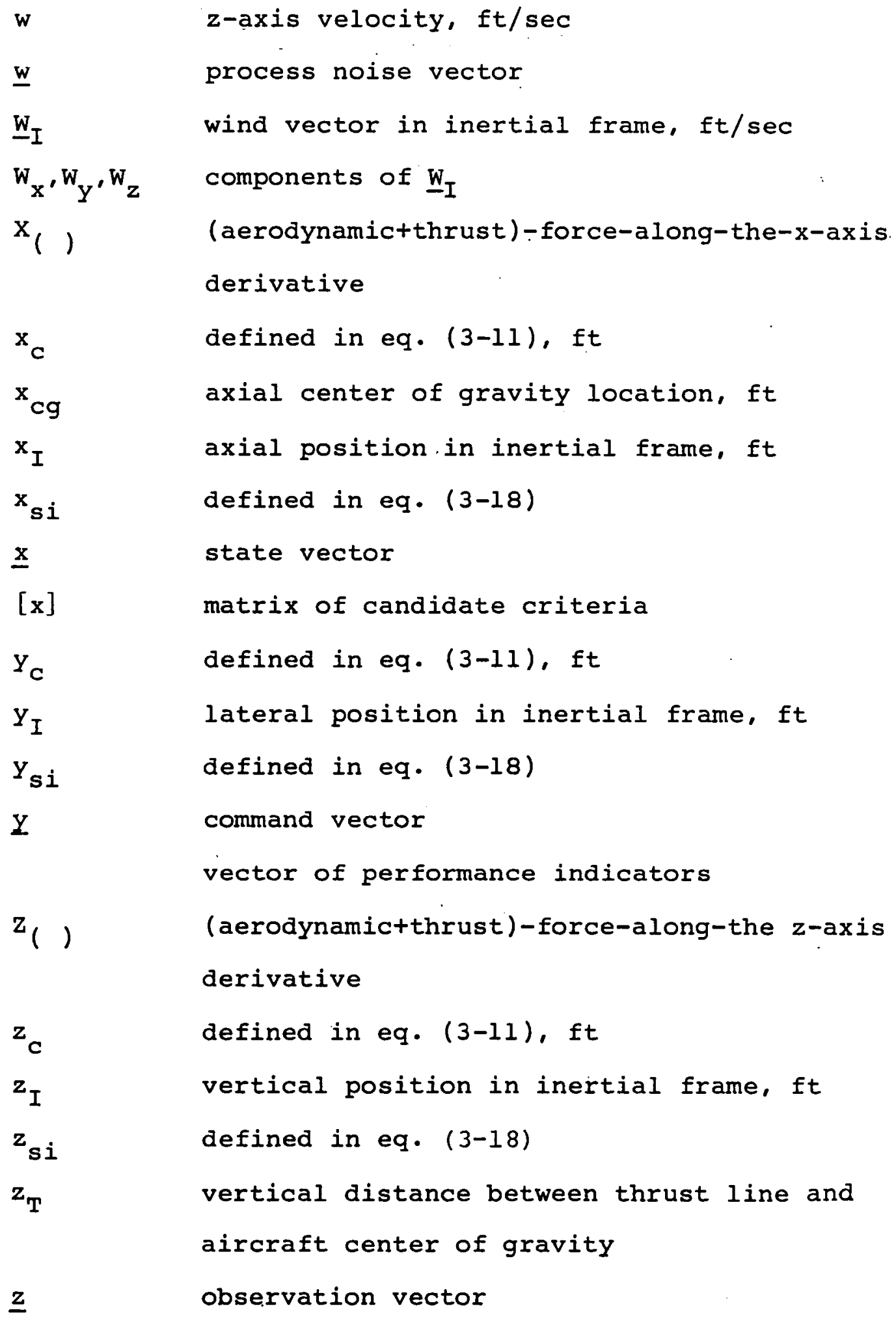


Variables (Greek)

a

$\boldsymbol{\beta}$

$\underline{\beta}$

$\boldsymbol{\gamma}$

$\delta \mathrm{E}$

$\delta$ e

$\delta \mathbf{F}$

$\delta T$

$\delta t$

$\epsilon$

$n_{p}$

$\theta$

$\Lambda$

$\lambda$

$\mu$

$\zeta_{\mathrm{p}}$

$\left(\zeta \omega_{n}\right)_{p}$

$\rho$

$\rho_{\mathrm{G}}$

$\Sigma$

$\sigma$ angle of attack, deg

statistical significance parameter

angle of sideslip, deg

vector of partial regression coefficients

vertical flight path angle, deg

definition in eq. (3-16)

elevator deflection, deg

longitudinal stick deflection, deg

flap deflection, deg

throttle setting, percent

throttle control, percent

Earth eccentricity (of the equator ellipse)

error in following a desired

value of a variable

propeller efficiency

pitch attitude angle, deg

wing sweep angle, deg

latitude, deg

longitude, deg

phugoid damping

phugoid total damping, rad/sec

air density, slugs/ft ${ }^{3}$

gaussian radius of curvature, ft

summation

measurement noise standard deviation

variance of the regression error 


$\begin{array}{ll}\tau & \text { rise time, sec } \\ \phi & \text { roll attitude angle, deg } \\ \Phi & \text { state transition matrix } \\ \psi \cdot & \text { yaw attitude angle, deg } \\ \Omega & \text { Earth rotation rate, rad/sec } \\ \omega & \text { frequency, rad/sec } \\ \tilde{\omega} & \text { body angular rate skew matrix } \\ \tilde{\omega}_{\mathrm{E}} & \text { definition in eq. (3-8) } \\ \omega_{\mathrm{np}} & \text { phugoid natural frequency, rad/sec }\end{array}$

\section{Superscripts}

B

I

$$
\begin{aligned}
& \text { transformation to body axes } \\
& \text { transformation to inertial axes }
\end{aligned}
$$

\section{Subscripts}

A

ARA

B

comm

EC

$\mathbf{F}$

f kinematic model A

Avionics Research Aircraft

body axis frame

from body axes (with transformation matrix)

kinematic model $B$

feedback

backward filter

commanded (desired) value

Earth-centered coordinate frame

feed forward

filtered 


\begin{tabular}{|c|c|}
\hline gs & glide slope \\
\hline k & sampling instant index \\
\hline I & inertial frame \\
\hline & $\begin{array}{l}\text { from inertial axes (with transformation } \\
\text { matrix) }\end{array}$ \\
\hline i & running index in statistical analysis \\
\hline Lo & for zero angle-of-attack \\
\hline M & model \\
\hline$\circ$ & $\begin{array}{l}\text { nominal value } \\
\text { initial value }\end{array}$ \\
\hline q & sensitivity to pitch rate \\
\hline $\mathbf{s}$ & smoothed \\
\hline si & navigation station sequencing index \\
\hline tr & trim wheel \\
\hline $\mathrm{u}$ & sensitivity to $x$-axis velocity \\
\hline w & sensitivity to z-axis velocity \\
\hline $6 \mathrm{E}$ & sensitivity to elevator deflection \\
\hline$\delta \mathbf{F}$ & sensitivity to flap \\
\hline$\delta \mathbf{T}$ & sensitivity to throttle deflection \\
\hline
\end{tabular}

Punctuation

$\begin{array}{ll}(\text { ) } & \text { derivative of quantity with respect to time } \\ (\text { ) } & \text { average value } \\ \text { (-) } & \text { vector quantity }\end{array}$




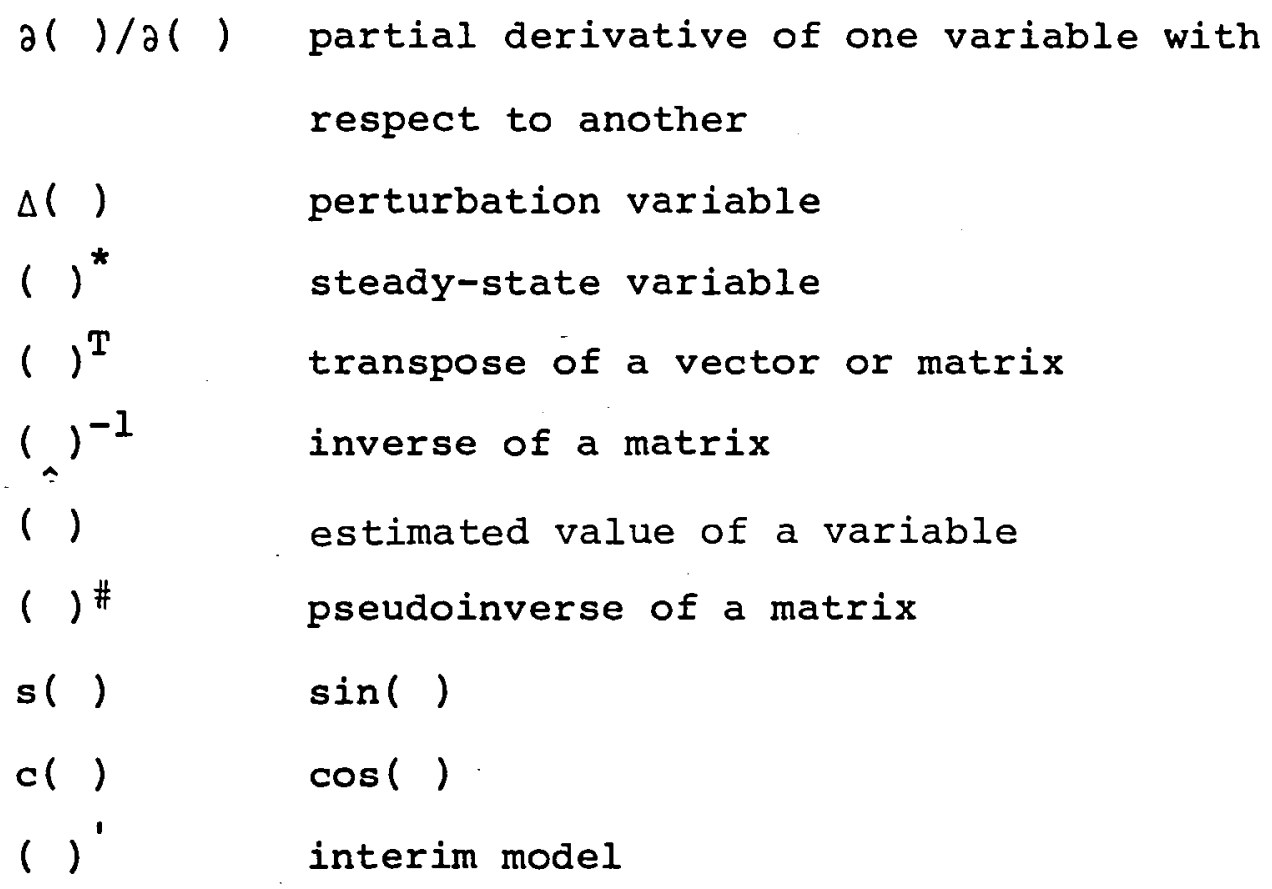

\section{Acronyms}

$\begin{array}{ll}\text { A/D } & \text { Analog-to-Digital } \\ \text { ADF } & \text { Automatic Direction Finder } \\ \text { ARA } & \text { Avionics Research Aircraft } \\ \text { BE } & \text { backward Elimination regression variant } \\ \text { CDU } & \text { Control Display Unit } \\ \text { CHR } & \text { Cooper-Harper Rating } \\ \text { COL } & \text { Colts Neck VoR/DME station } \\ \text { D/A } & \text { Digital-to-Analog } \\ \text { DARE } & \text { Digital Avionics Research system } \\ \text { DME } & \text { Distance Measuring Equipment } \\ \text { FAA } & \text { Federal Aviation Administration } \\ \text { FAR } & \text { Federal Aviation Regulations } \\ \text { FBW } & \text { Fly-By-Wire } \\ \text { FRL } & \text { Flight Research Laboratory } \\ & \end{array}$




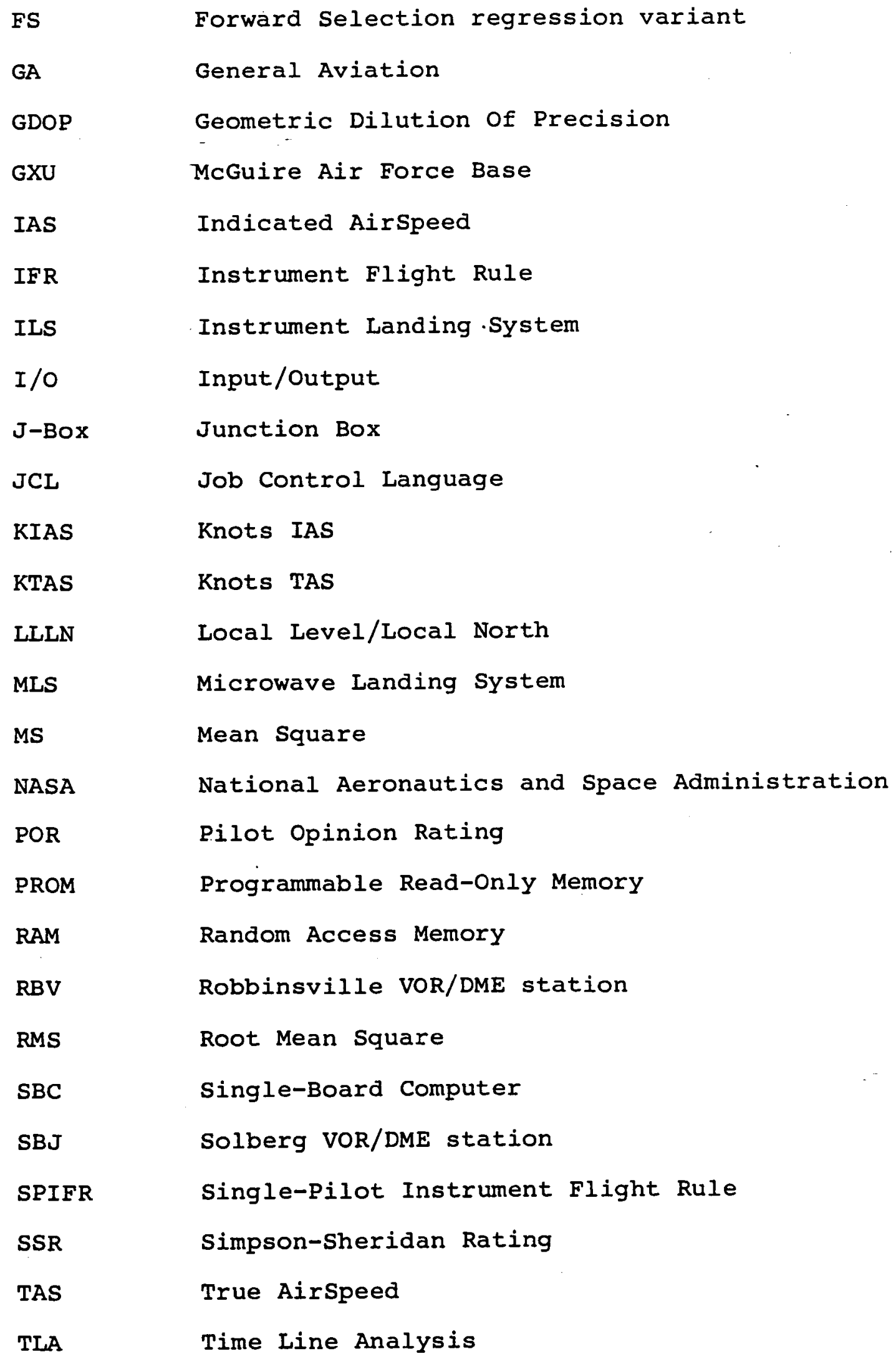


TCV

TVC

VFR

VOR

Terminal Configured Vehicle

Thrust Vector Control

Visual Flight Rule

Very-high-frequency Omni-Range 
Chapter 1

INTRODUCTION

\subsection{BACKGROUND}

A large portion of air transportation activity is carried out by General Aviation (GA) aircraft, which are light aircraft often flown by a single pilot. Currently, there are about 200,000 active GA aircraft which fly over 40 million hours per year, and the projected number of active GA aircraft in 1990 is about 300,000 (Ref. 1). Although the potential exists for GA operations to be of even greater value in satisfying air transportation needs, this potential may not be realized without substantial improvements in the safety, efficiency, and relative cost of GA aircraft. The maturing microprocessor technology may help achieve these goals, as in the computation of fuel-efficient cruise (Ref. 2).

This research addressed the safety problem of single-Pilot Instrument Flight Rule (SPIFR) operations. A statistical analysis of fatal GA accidents (Ref. 3) reveals that most of them occur under Instrument Flight Rule (IFR) conditions. 
The major factors which contribute to an increased hazard in SPIFR operations are associated with the serious jump in the pilot's workload when going from Visual Flight Rule (VFR) to IFR conditions. As pointed out in Ref. 4, these factors belong to two distinct categories: (I) deficient aircraft dynamic response, and (2) inadequate navigation/communication, control and display systems. The work reported in this reference results in a recommendation to introduce a flight-director display combined with an attitude-command control system for the glide-slope segment of the SPIFR mission. It constitutes an example of the effort, pursued recently also by other research teams, to apply modern technology to alleviation of the problems of the second category. These research workers assume that the latter is the dominant factor in pilot workload under SPIFR conditions. Very little has been done to validate this assumption and to challenge, if necessary, the deficiencies of the aircraft's dynamic response.

Deficiencies that are particularly relevant to SPIFR workload, are trim-related or low-frequency response characteristics. This is a response region for which it is difficult to obtain quantitative flying qualities criteria. Indeed, Federal Aviation Administration (FAA) airworthiness standards for light airplane design (Ref. 5) provide only qualitative guidelines and they address only the short-peri- 
od oscillations when referring to longitudinal dynamic stability! Until recently, there has been. little incentive to change this situation, as GA pilots flying VFR could cope with mild divergences. On the other hand, the military light airplanes had to perform additional tasks such as observation and consequent communications, which increased substantially the pilot's workload and required a more "well-behaved" aircraft dynamic response. References 6, 7, and 8 represent a research effort which led to the inclusion of explicit phugoid-related (long-period) flying qualities criteria into the military specifications (Ref. 9). For example, the phugoid damping ratio has been restricted to $\zeta_{p} \geqslant 0.04$ for normal (Level I) flight (it can be unstable in Level 3 ).

With the expansion of both VFR and IFR activity in recent years, more attention has been focused on flying qualities criteria for light airplanes. References 10,11 and 12 represent extensive research in this area conducted in the Flight Research Laboratory of Princeton University. While Ref. 10 and 11 emphasize the short-period response, Ref. 12 elaborates on all aspects of longitudinal dynamics. For example, it analyzes the effects of downsprings which are often installed to achieve stable force gradients, and concludes that such a modification in aircraft control design may destabilize the long-period oscillation. Finally the 
authors comment: "Although this is allowed by the regulations, it appears as a tendency for the airplane to wander in speed, pitch attitude and altitude, and the need for constant stabilization adds to the pilot workload, particularly when flying on instruments." This is a qualitative statement, but it is indicative of the growing awareness of the need to analyze the GA SPIFR flight regime.

This recognition has led several researchers to employ digital computers and ground-based simulators in investigation of this flight regime. Hinton and Shaughnessy of the Langley Research Center of the National Aeronautics and Space Administration (NASA) have looked into the adaptation of the Time Line Analysis (TLA) to SPIFR workload assessment (Ref. 13). Bergeron, also of Langley, studied several levels of autopilot improvement for. General Aviation, and discovered that an excessively automated mode may be more susceptible to human operator exror (Ref. 14). Reference I5 addressed the problem of optimal instrument location and of visual scanning workload, using oculometer measurements. The results in Ref. 16 indicate that performance in pathfollowing tasks improves with increase in display sensitivity. All these references simulate complete SPIFR missions with the assumption that the cockpit systems workload is dominant. 
This key assumption must be evaluated, and if there are significant flying qualities criteria, which represent appreciable pilot workload due to dynamic deficiencies, they should be identified. For ultimate credibility, these answers must be backed by statistically significant results obtained via extensive flight testing under SPIFR conditions. Flight testing is the only way to achieve completely realistic motion cues, which are rarely matched in ground-based simulation; also emotional stress and environmental factors are most accurately portrayed in flight. Such a research effort should not rely solely on subjective Pilot Opinion Rating (POR) scales in evaluating flying qualities criteria. Its conclusions may be more sound if crosschecked with computed performance indicators based on objective measurements of pilot performance while performing typical SPIFR tasks, e.g., glide slope tracking or airspeed holding.

\subsection{GOALS AND SIGNIFICANT RESULTS}

The primary objective of this research was to determine the relative importance of the aircraft longitudinal dynamic response versus the navigation/communication workload and to identify quantitative and statistically significant flying qualities criteria for GA SPIFR operations. A flight test program involving in-flight simulation of realistic SPIFR 
tasks and of à range of GA dynamic configurations was conducted to achieve this objective. As a result, several guidelines are being proposed for GA flying qualities criteria:

* An explicit lower bound is suggested for the phugoid damping ratio. Its value, 0.04 , is consistent with the criterion of the military flying qualities specifications.

* This research identified an upper bound for the stick force sensitivity to airspeed change, which is addressed by the FAA regulations only qualitatively; $\Delta \mathrm{f}_{\mathrm{s}}^{*} / \Delta \mathrm{V}_{\mathrm{comm}} \leqslant 1.4 \mathrm{lb} / \mathrm{kt}$. Any quantification of this criterion is important, as it is equivalent to a perception by the pilot of having direct control of velocity.

* A new criterion, the pitch attitude sensitivity to airspeed change from one trimmed flight condition to another, $\Delta \theta^{*} / \Delta V_{\text {comm }}$ is suggested. Its value should not be lower than $-0.7 \mathrm{deg} / \mathrm{kt}$.

These results were obtained using modern control and estimation theory and statistical algorithms, combined with realistic in-flight simulation of aerodynamic configurations and SPIFR missions, and accurate data analysis. Those aerodynamic configurations which affect most directly the lowfrequency dynamic and trim-related airframe response were 
identified analytically. The range of variation for each of these aerodynamic coefficients was chosen to cover both the existing light aircraft fleet and recent trends in GA design. For each of the chosen configurations, frequency- and time-domain-related candidate flying qualities criteria were derived.

To provide experimental measures of performance under SPIFR conditions, the chosen aerodynamic configurations were implemented on Princeton's Avionics Research Aircraft (ARA), which is capable of simulating in flight a wide range of aircraft dynamic responses. A microprocessor-based digital data acquisition system was developed and installed, along with a new avionics package. ${ }^{1}$ planning of the navigational trajectories and the experiment design were based on theoretical and statistical considerations.

To assure accurate computation of performance indicators, a numerically robust algorithm for optimal flight path reconstruction was developed. This algorithm uses as inputs the outputs of a microprocessor-based multiple DME (Distance Measurement Equipment) scanner along with aerodynamic and inertial measurements. This integrated flight testing and trajectory estimation methodology, which uses low-cost in-

1 Details of the ARA and the development of the integrated avionics and data recording system are given in Appendix A. 
strumentation, was demonstrated in flight and may be readily applied " to flight technical error evaluation of other navigational systems.

\subsection{ORGANIZATION OF THE REPORT}

Chapter 2 presents the derivation of candidate flying qualities criteria for SPIFR operations and the theoretical aspects of the methodology of SPIFR flight-test design. Chapter 3 discusses the application of optimal estimation theory to flight-path reconstruction and its implications on the methodology of the flight-test design. Chapter 4 elaborates on the processing of the experimental results, on the deduction of performance indicators, and on the derivation of SPIFR flying qualities criteria. Conclusions and recommendations are presented in Chapter 5. The experimental system, the ARA in-flight simulator, and the onboard microprocessor-based data acquisition complex, are described in Appendix A. A detailed derivation of SPIFR candidate criteria is elaborated in Appendix B. Appendix C contains the derivation of the linearized version of the mathematical model, required for the optimal flight path estimation. Appendix $D$ covers aspects of the flight-test procedure and summarizes the complete post-flight processing, whose efficiency becomes an important factor when applied to a 40-hour flight-test series. Experimental pilot opinion rating and 
commentary data (raw data) are compiled in Appendix E. The relevant FORTRAN and Job Control Language (JCL) code listings were collected in Ref. 17. 


\section{Chapter 2 \\ DERIVATION OF CANDIDATE FLYING QUALITIES \\ CRITERIA AND SPIFR EXPERIMENTAL DESIGN}

This chapter starts with the formulation of the aircraft dynamic model and of its linearization, which are required for the derivations in the subsequent sections. Section 2.2 discusses the identification of SPIFR aerodynamic configurations and of candidate flying qualities criteria. The simulation of the chosen configurations on the ARA using the implicit model following algorithm is presented in section 2.3. SPIFR mission planning (Section 2.4) is based on mathematical-statistical modeling of the en-route navigational errors. Finally, preliminary flights to verify the system are described in Section 2.5 .

\subsection{AIRCRAFT DYNAMIC MODEL}

The general formulation of a nonlinear dynamic model of a system is,

$$
\underline{\dot{x}}=\underline{f}(\underline{x}, \underline{a})+\underline{w}
$$

where $\underline{x}$ is the state vector and $\underline{a}$ is the control vector. The effect of the vector of disturbances, $\underline{w}$ will be discussed in Chapter 3. The state vector $\underline{x}$ used here contains 
three components each of translational rate $(u, v, w)$, translational position $\left(x_{I}, y_{I}, z_{I}\right)$, angular rate $(p, q, r)$ and angular attitude $(\phi, \theta, \psi)$. Both body and inertial axis frames are right-handed with $z$ pointing downward. The $x_{B}$ body axis is aligned with the airplane center line and is positive forward of the center of gravity, which is the origin of the body frame. The inertial Cartesian frame suitable to the derivations of this chapter is the Local Level/ Local North (LLLN) axis system. The Forrestal airfield reference point has been chosen as its origin, the $x_{I}$ axis points toward North and $z_{I}$ points toward the Earth center. For accurate flight path reconstruction, the LLLN frame has to be defined with respect to a new Earth-centered Cartesian axis system. This issue is elaborated in Chapter 3.

The translational rate equation of the aircraft mathematical model is,

$$
\dot{\underline{v}}_{\mathrm{air}}=\underline{\mathrm{a}}_{\mathrm{B}}+\tilde{\omega}_{\mathrm{V}} \underline{\mathrm{air}}+\mathrm{H}_{\mathrm{I}}^{\mathrm{B}} \underline{g}_{\mathrm{I}}
$$

The airspeed, expressed in body axes, is,

$$
\underline{v}_{\text {air }}=\left[\begin{array}{lll}
u & \\
\end{array}\right]^{T}
$$

Acceleration, expressed in body axes, is,

$$
\underline{a}_{B}=\left[a_{x} a_{y} a_{z}\right]^{T}
$$


The angular rate cross-product-equivalent matrix $\tilde{\omega}$ is defined as,

$$
\tilde{\omega} \triangleq\left[\begin{array}{rrr}
0 & r & -q \\
-r & 0 & p \\
q & -p & 0
\end{array}\right]
$$

The gravity vector in the LLLN inertial axis system is,

$$
\underline{\mathrm{g}}_{\mathrm{I}}=\left[\begin{array}{lll}
0 & 0 & \mathrm{~g}
\end{array}\right]^{\mathrm{T}}
$$

The transformation matrix $H_{I}^{B}$ from inertial (I) to body (B) axes, with $(\phi, \theta, \psi)$ Euler rotations in the specified order, is,

$$
H_{I}^{B} \triangleq\left[\begin{array}{lcc}
c \psi c \theta & s \psi c \theta & -s \theta \\
c \psi s \theta s \phi-s \psi c \phi & s \psi s \phi s \theta+c \psi c \phi & c \theta s \phi \\
c \psi s \theta c \phi+s \psi s \phi & s \psi s \theta c \phi-c \psi s \phi & c \theta c \phi
\end{array}\right]
$$

where

$$
\begin{aligned}
& s() \triangleq \sin () \\
& c() \triangleq \cos ()
\end{aligned}
$$

The second equation of the aircraft motion 6-DOF mathematical model describes the transformation of body-axis rates to Euler angle rates, and it is, 


$$
\left[\begin{array}{l}
\dot{\phi} \\
\dot{\theta} \\
\dot{\psi}
\end{array}\right]=\mathrm{I}_{\mathrm{B}}^{\mathrm{I}} \cdot\left[\begin{array}{l}
\mathrm{p} \\
\mathrm{q} \\
\mathrm{r}
\end{array}\right]
$$

where

$$
I_{B}^{I} \triangleq \frac{I}{c \theta}\left[\begin{array}{ccc}
c \theta & s \theta s \phi & s \theta c \phi \\
0 & c \theta c \phi & -c \theta s \phi \\
0 & s \phi & c \phi
\end{array}\right]
$$

The third dynamic equation combines the effects of airspeed $\underline{V}_{\text {air }}$ and of the wind vector $\underline{W}_{I}$ (expressed in inertial axes) to compute. translational rate,

$$
\dot{\mathrm{x}}_{\mathrm{I}}=\mathrm{H}_{\mathrm{B}}^{\mathrm{I}} \underline{\mathrm{air}}_{\mathrm{ar}}+\underline{\mathrm{W}}_{\mathrm{I}}
$$

where $\underline{x}_{I}$ is the position vector expressed in inertial axes,

$$
\underline{x}_{I}=\left[x_{I} y_{I} z_{I}\right]^{T}
$$

Based on the orthonormality of $\mathrm{H}_{I}^{B}$ in eq. (2-7),

$$
\mathrm{H}_{\mathrm{B}}^{\mathrm{I}}=\left(\mathrm{H}_{\mathrm{I}}^{\mathrm{B}}\right)^{-1}=\left(\mathrm{H}_{\mathrm{I}}^{\mathrm{B}}\right)^{\mathrm{T}}
$$

The following relationships constitute the algebraic part of the model, yielding the output or the measurement models. The airspeed absolute value is, 


$$
v=\left|\underline{v}_{a i r}\right|=\left(u^{2}+v^{2}+w^{2}\right)^{1 / 2}
$$

The angle of attack is given by,

$$
\alpha=\tan ^{-1}(w / u)
$$

The sideslip angle definition used here is,

$$
\beta=\tan ^{-1}(v / u)
$$

The angle-of-attack definition is entirely conventional, but the sideslip angle definition differs from the conventional definition, which is,

$$
\beta=\sin ^{-1}(v / v)
$$

The purpose for using eq. (2-16) rather than eq. (2-17) is to assure compatibility with the actual mechanization in the $A R A ;$ the zero position of the $\beta$-vane is parallel to the $x_{B}$ body axis, and its rotation axis is parallel to the $z_{B}$ body axis.

Assuming that the origin of the inertial frame is at sea level, the altitude $\mathrm{h}$ is,

$$
\mathrm{h}=-\mathrm{z}_{\mathrm{I}}
$$

The acceleration vector $\underline{a}_{B}$ of eq. (2-2) and (2-4) reflects the effect of aerodynamic and thrust forces acting on the airframe. 
To examine the effects of the low-frequency longitudinal dynamics and trim-related response on SPIFR performance, the ARA was used as an in-flight simulator. The capabilities of this experimental vehicle and their enhancements, introduced for the purposes of this research, are described in detail in Appendix A. Its nonlinear dynamic model (Ref. 18) constitutes a submodel of the general formulation in eq. (2-1) to $(2-18)$ :

$$
\begin{aligned}
& \dot{u}=a_{x}-q w-g s \theta=(\overline{q S} / m) c_{x}\left(\alpha, T_{C}^{\prime}, \delta F\right)-q w-g s \theta(2-19) \\
& \dot{w}=a_{z}+q u+g c \theta=\overline{(q S / m) C}\left(\alpha, T_{c}^{\prime}, \delta F, \delta E, q\right)+ \\
& q u+g c \theta \\
& \dot{q}=\left(\overline{q S c} / I_{Y Y}\right) C_{m}\left(\alpha, T_{c}^{\prime}, \delta E, q, \dot{w}\right) \\
& \dot{\theta}=\mathrm{q} \\
& \dot{\mathrm{x}}_{\mathrm{I}}=\mathrm{uc} \theta+\mathrm{ws} \theta \\
& \dot{\mathrm{z}}_{\mathrm{I}}=-\mathrm{us} \theta+\mathrm{wc} \theta
\end{aligned}
$$

Accompanying relationships are,

$$
\begin{aligned}
& \alpha=\tan ^{-1}(w / u) \\
& \bar{q}=0.5 v^{2} \\
& v=\left(u^{2}+w^{2}\right)^{1 / 2}
\end{aligned}
$$




$$
\begin{aligned}
& \mathrm{T}_{\mathrm{c}}^{\prime}=\mathrm{T}_{\max } / \overline{\mathrm{qS}}=\mathrm{k}_{\mathrm{T}} \mathrm{T} / \mathrm{V}^{3} ; \mathrm{k}_{\mathrm{T}}=2 \eta_{\mathrm{p} \mathrm{P}_{\max } / \rho \mathrm{S}} \\
& c_{x}=C_{x}(\alpha)+C_{x \delta F}(\alpha) \delta F+T_{c}^{\prime} \\
& C_{z}=C_{z O}+C_{z_{a}}^{\alpha}+C_{z \delta F} \delta F+C_{z \delta E} \delta E ;(\alpha<14.3 \operatorname{deg})(2-30) \\
& c_{m}=c_{m o}+c_{m_{a}}^{\alpha}+c_{m q}(0.5 \bar{c} / v) q+c_{m \delta E}\left(1+x_{c g} / \bar{I}_{t}\right) \delta E \\
& +\left(x_{c g} / \bar{c}\right) c_{z}+\left(z_{T} / \bar{c}\right) T_{c}^{\prime}
\end{aligned}
$$

In these equations $m$ is the aircraft mass (slugs), $I_{Y Y}$ is the pitching moment of inertia (slugs-ft ${ }^{2}$ ), $s$ is the wing (reference) area $\left(f t^{2}\right), \eta_{p}$ is the propeller efficiency, and $\mathrm{P}_{\max }$ is the maximum power available. $\mathrm{T}_{\max }$ is the maximum thrust (for throttle travel $\delta \mathrm{T}$ of $100 \%$ ) available with $\mathrm{P}_{\max }$ (lb), $\bar{c}$ is the (reference) mean aerodynamic chord length (ft), $x_{c g}$ is the axial center of gravity location with respect to the reference point $(f t), z_{T}$ is the vertical (in body axes) distance between the thrust line and the aircraft center of gravity; and $\bar{l}_{t}$ is the distance between wing and horizontal tail centers of pressure (ft). The aerodynamic coefficient $c_{m q}$ is augmented to account for the $\dot{\alpha}$-effect, $\delta E$ is the elevator deflection and $\delta F$ is the flap deflection.

For analysis purposes, the above model can be linearized about a nominal trimmed flight equilibrium. Given airspeed and altitude, these flight trim values were found by minimizing a quadratic function of $\dot{u}, \dot{w}$ and $\dot{q}(\operatorname{Ref} .19)$. At this function's minimum: 


$$
\begin{aligned}
& \dot{\mathrm{u}}=\dot{\mathrm{w}}=\dot{\mathrm{q}}=\mathrm{q}=0 \\
& \theta=\alpha(\gamma=0, \text { straight and level flight })
\end{aligned}
$$

As reported in Ref. 20, the full-scale Navion aircraft was tested in a wind tunnel with an electrical motor actually driving the propeller at several power settings. These data were crosschecked with results of numerous flight tests. This provides a sound basis for the theoretical analysis.

With the SPIFR-related onboard systems, the ARA longitudinal inertial data are,

$$
\begin{aligned}
& m=93.17 \text { slugs }=3000 \mathrm{lb} \\
& I_{Y Y}=2829.5 \text { slug- } \mathrm{ft}^{2}
\end{aligned}
$$

All configurations were flown within an airspeed range of 75 knots to 105 knots and at altitudes up to $3000 \mathrm{ft}$. Thus, for configuration matching and candidate SPIFR criterion deduction, a typical flight equilibrium condition of 75 knots and $2000 \mathrm{ft}$ was chosen:

$$
\begin{aligned}
\mathrm{h} & =2000 \mathrm{ft} ; \rho_{2000} / \rho_{\mathrm{sl}}=0.985, \mathrm{~V}_{\mathrm{sl}} / \mathrm{V}_{2000}=0.97(2-36) \\
\mathrm{V} & =75 \mathrm{KIAS}=130.6 \mathrm{fps}(\mathrm{TAS}) \\
\mathrm{T}_{\max } & ={ }_{\mathrm{p}} \mathrm{P}_{\operatorname{maxSL}} \rho_{2000} / \rho_{\mathrm{SL}} / \mathrm{V}=0.8 \times 156750 \times 0.985 / 130.6 \\
& =945 \mathrm{Ib}
\end{aligned}
$$




$$
T_{C}^{\prime}\left(\delta T^{\prime}=1008\right)=T_{\max } /(\overline{q S})=945 / 3570=0.265
$$

Finally, the level flight trim values are,

$$
\begin{aligned}
& \alpha=\theta=8.77 \mathrm{deg} \\
& \delta E=4.5 \mathrm{deg} \\
& \delta T=49.48
\end{aligned}
$$

Linearization of the equations of motion provides a useful means for assessing the stability of the aircraft and its response to small perturbations. Expanding the system mathematical model in a Taylor series with respect to the above set of nominal conditions and disregarding the nonlinear terms (Ref. 21),

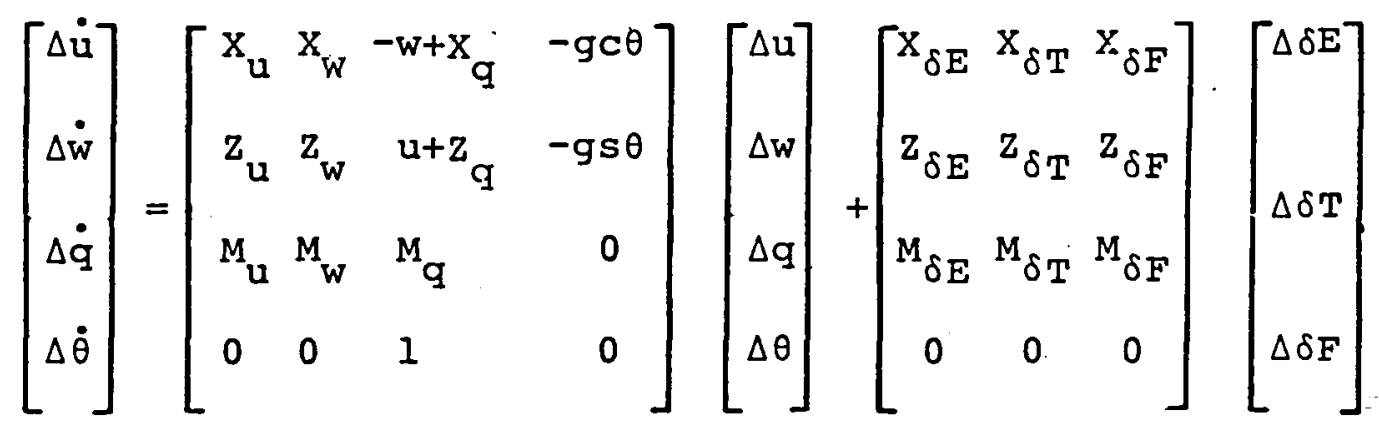

The translational position variables were omitted from this formulation. The range, $x_{I}$ does not affect the aircraft dynamics, so its eigenvalue is identically zero. The 
height mode (Ref. 21) which arises from the altitude dependency on air density, also is of no consequence under SPIFR conditions. The ARA's height mode has a stable time constant of about $1000 \mathrm{sec}$, which is substantially longer than the time scale of the phugoid mode. Equation $(2-43)$ is of the form of a state equation,

$$
\underline{\Delta x}=F \underline{\Delta x}+G \underline{a}
$$

where $F$ is the state matrix, $G$ is the control matrix, $\Delta x$ is the state vector and $\underline{\Delta u}$ is the control vector. $F$ and $G$ are the Iinearization Jacobians,

$$
\begin{aligned}
& F=\underline{\partial f} /\left.\underline{\partial x}\right|_{\underline{x}=\underline{x} 0, \underline{u}=\underline{u} 0} 0 \\
& G=\underline{\partial f} /\left.\underline{\partial u}\right|_{\underline{x}=\underline{x} 0, \underline{a}=\underline{u} 0}
\end{aligned}
$$

As obvious from eq. $(2-43)$, they consist of the conventional stability-and-control derivatives. The matrix $F$ is useful, as its eigenvalues predict the local stability of the flight path motion (Ref. 22). For example, eigenvalue $\lambda_{i}$, of a dynamic response mode $i$, provides both the natural frequency and the damping ratio of this mode,

$$
\begin{aligned}
& \omega_{n i}=\left|\operatorname{Re}_{\lambda}^{2}+\operatorname{Im}_{\lambda}^{2}\right|_{i}^{1 / 2} \\
& \left(\zeta \omega_{n}\right)_{i}=\left|\operatorname{Re} \lambda_{\lambda i}\right|
\end{aligned}
$$




$$
\zeta_{i}=\left(\zeta \omega_{n}\right)_{i} / \omega_{n i}
$$

The numerical values of the $F$ and $G$ elements (eq. (2-43)), based on the chosen nominal flight equilibrium are,

$$
\begin{aligned}
& x_{u}=-0.083 \mathrm{sec}^{-1} \\
& x_{w}=+0.094 \mathrm{sec}^{-1} \\
& -w+X_{q}=-v s \alpha=-19.95 \text { fps } \\
& x_{\theta}=-g c \theta=-31.7 \mathrm{ft} / \mathrm{sec}^{2} \\
& z_{u}=-0.549 \mathrm{sec}^{-1} \\
& z_{w}=-1.30 \mathrm{sec}^{-1} \\
& \mathrm{u}+\mathrm{z}_{\mathrm{q}}=\mathrm{Vc} \alpha=129.1 \mathrm{fps} \\
& z_{\theta}=-g s \theta=-5.25 \mathrm{ft} / \mathrm{sec}^{2} \\
& M_{u}=+0.0037 \mathrm{ft}^{-1}-\sec ^{-1} \\
& M_{w}=-0.065 \mathrm{ft}^{-1}-\mathrm{sec}^{-1} \\
& M_{q}=-2.124 \mathrm{sec}^{-1} \\
& \mathrm{x}_{\delta \mathrm{E}}=0 \\
& z_{\delta E}=-0.089 \mathrm{sec}^{-1} \\
& M_{\delta E}=-9.82 \sec ^{-2} \\
& x_{\delta T}=+0.17\left(\mathrm{ft} / \mathrm{sec}^{2}\right) / \mathrm{mm}
\end{aligned}
$$




$$
\begin{aligned}
& \mathrm{Z}_{\delta_{T^{\prime}}}{ }^{\mathrm{M}_{\delta_{T^{\prime}}}} \mathrm{M}_{\delta_{\mathrm{F}}}=0 \\
& x_{\delta_{F}}=-0.242 \mathrm{ft} / \mathrm{sec}^{2} \\
& z_{\delta_{F}}=-34.7 \mathrm{ft} / \mathrm{sec}^{2}
\end{aligned}
$$

\subsection{IDENTIFICATION OF SPIFR AERODYNAMIC CONFIGURATIONS AND CANDIDATE FLYING QUALITIES CRITERIA}

Identification of the aerodynamic parameters that are most likely to affect airplane performance and pilot workload under SPIFR conditions, constitutes an important aspect of this research. The analytical tools, used both to identify the SPIFR aerodynamic configurations ${ }^{l}$ and to derive the candidate flying qualities criteria, include eigenvalue or frequency/damping computations and examination of transient and steady-state airframe characteristics. Consequently, both time- and frequency-domain metrics were considered. The eigenvalues were computed based on F (eq. (2-43)) and the computation of the transient characteristics (e.g., overshoots and rise times following a control step input) involved linear interpolation of tabulated data and numerical integration (fourth-order Runge-Kutta algorithm; Ref. 23)

1 As used here, a configuration is a set of aerodynamic coefficients that characterizes the dynamic response of an aircraft. 
of the set of nonlinear differential equations presented in the previous section. For the SPIFR flight regime, the long-period motion component of these results may be expected to be of most interest; the short-period, which is the higher frequency mode, is well separated from the phugoid for the GA-type airplanes.

With regard to the steady-state characteristics, an important feature of the SPIFR flight regime is that the pilot often is required by Air Traffic Control (ATC) to change altitude or airspeed. Each such flight path variable change is produced by a combination of thrust and horizontal control surface adjustments. A configuration which requires large control deflections to retrim from one flight equilibrium to another may seriously increase the pilot's workload under already-strained SPIFR conditions. The output command algorithm (Ref. 24) constitutes the most suitable analytical tool for the identification of such critical configurations. Its mathematical formulation is based on adding an output equation to the state equation, eq. (2-44) of the previous section,

$$
\begin{aligned}
& \underline{\Delta x}=F \Delta x+G \Delta \underline{\Delta u} \\
& \Delta y=H_{x} \underline{\Delta x}+H_{u} \underline{\Delta u}
\end{aligned}
$$

$\Delta y$ represents the desired flight-path variations. $\mathrm{H}_{\mathrm{x}}$ and $\mathrm{H}_{\mathrm{u}}$ are output matrices. Solving these two equations simultane- 
ously, both the required state and control perturbations are found,

$$
\left[\begin{array}{c}
\underline{\Delta x}^{*} \\
\underline{\Delta u}^{*}
\end{array}\right]=\left[\begin{array}{cc}
F & G \\
& \\
H_{x} & H_{u}
\end{array}\right]^{-1}\left[\begin{array}{c}
0 \\
\Delta y_{c o m m}
\end{array}\right]
$$

or,

$$
\left.\begin{array}{l}
0=\underline{F x}^{*}+\underline{G u}^{*} \\
\underline{\Delta y_{\mathrm{COmm}}}=\mathrm{H}_{\mathrm{x}} \underline{\Delta \mathrm{x}^{*}}+\mathrm{H}_{\mathrm{u}} \underline{\underline{\Delta u}} *
\end{array}\right\}
$$

()$^{*}$ symbolizes the steady-state variations in state and control that correspond to $\Delta_{\mathrm{comm}}$. The solution to eq. $(2-70)$ is,

$$
\begin{aligned}
& \underline{\Delta x}^{*}=-F^{-1} G S \Delta \underline{y}_{c o m m} \\
& \underline{\Delta u}^{*}=S \Delta y_{C o m m}
\end{aligned}
$$

where

$$
S=\left(-H_{x} F^{-1} G+H_{u}\right)^{-1}
$$

Conversely, the aircraft response to the pilot's commands after the decay of all transients is obtained from eq. (2-44) by setting $\underline{\dot{x}}=0$, 


$$
\underline{\Delta x^{*}}=-F^{-1} \underline{G \Delta}_{c o m m}
$$

As a result-of preliminary analysis the following aerodynamic parameters received priority for SPIFR flight testing:

$$
\begin{aligned}
& \text { (a) } x_{u^{\prime}}, z_{u^{\prime}} z_{w^{\prime}} M_{u} \\
& \text { (b) } z_{\delta E^{\prime}} z_{\delta T}, M_{\delta T}
\end{aligned}
$$

These stability and control derivatives fall into two categories: those that affect only trim and those that affect both trim and stability. Control derivatives listed in (b) fall into the first category because, as demonstrated by eq. (2-43), they appear in the control matrix G, thus affecting $\underline{\Delta x^{*}}$ and $\underline{\Delta a^{*}}$. Stability derivatives listed in (a) fall into the second category because they appear in the $F$ matrix, thus affecting both trim and stability.

Now let us elaborate on the physical significance of changing each of the chosen aerodynamic coefficients and on deduction of candidate flying qualities criteria for GA SPIFR operations. The $z_{u}$ derivative is the equivalent of $-T L_{v}$, which is defined with respect to the flight path (hence not exactly identical to $z_{u}$ ). As follows from classical airplane stability theory (Ref. 25), the square of the phugoid natural frequency, ' $\omega_{n p}$ ' may be approximated as a linear function of $\mathrm{TL}_{\mathrm{V}}$ ' 


$$
\omega_{n p}^{2}=g\left(T L_{V} / V\right)=2(g / V)^{2}
$$

Accounting for compressibility but not for the generally negligible thrust effect on $\mathrm{TL}_{\mathrm{V}}$ '

$$
\begin{aligned}
T L_{V} \cong & (g / V)\left(2+C_{L_{V}} / C_{L}\right)= \\
& (g / V)\left[2+\left(1-C_{L_{O}} / C_{L}\right) M^{2} /\left(1-M^{2}\right)\right]
\end{aligned}
$$

As may be observed from eq. (2-75) and (2-76), for low-speed GA aircraft $\omega_{n p}$ is proportional to the inverse of $v$. Thus flying configurations with a range of $\mathrm{z}_{\mathrm{u}}$-values provides data for both slow aircraft and for small jets, which often are involved in SPIFR operations. The latter achieve higher airspeeds and may feature nonzero zero-lift coefficients $\mathrm{C}_{\mathrm{L}_{\mathrm{O}^{\prime}}}$ which tends to offset the compressibility effect (eq. $(2-76))$.

The $\mathrm{x}_{\mathrm{u}}$ derivative is the equivalent of $-\mathrm{TD}_{\mathrm{V}}$; as shown in Ref. 25, the phugoid damping $\zeta_{p}$ is a linear function of this coefficient,

$$
\zeta_{p}=-0.5 T D_{V} / \omega_{n p}
$$

Substituting from eq. (2-75),

$$
\zeta_{\mathrm{p}}=-0.35 \mathrm{TD} \mathrm{v} /(\mathrm{g} / \mathrm{v})
$$

As a design parameter, $-\mathrm{TD}_{\mathrm{V}}$ may be approximated by, 


$$
\begin{gathered}
-T D_{V} \cong 2(g / V)\left(C_{D} / C_{L}\right)=2(g / V)(L / D)^{-1}= \\
=2(g / V)(1 / \pi e)\left(C_{L} / A R\right)
\end{gathered}
$$

Thus configurations with low values of $\mathrm{x}_{\mathrm{u}}$ may represent $\mathrm{ul-}$ tra-light aircraft designs or airplanes with high aspect ratios. As suggested in Chapter 1, excessively low phugoid damping values may be problematic under SPIFR conditions. Due to the contribution of the compressibility effect, the $\mathrm{x}_{\mathrm{u}}$ derivative increases for higher-airspeed aircraft. This may be important in small jet designs; for propeller-driven airplanes the phugoid damping increases due to the power effect.

Special circumstances or control design considerations may also affect $\zeta_{p}$ As pointed out in Ref. 26, the wind shear effect tends to destabilize the phugoid and, as noted in Chapter 1, this is true also with regard to the installation of downsprings. Thus, by flying SPIFR missions with a wider range of $x_{u}$ or $\zeta_{p}$ configurations, we gain insight into these effect as well.

The $\mathrm{z}_{w}$ derivative represents the "lift curve slope" effect. Reference 25 provides an empirical fit for the lift coefficient for the entire range of wing aspect ratios,

$$
c_{L_{a}} \cong \pi \mathbb{R} /\left(1+\left[1+(.5 \mathbb{R} / c \Lambda)^{2}\left(1-M^{2} c^{2} \Lambda\right)\right]^{1 / 2}\right)
$$


As obvious from eq. (2-80), increasing the values of such design parameters as AR or cruise airspeed, improves the aerodynamic efficiency of a configuration; wing sweep tends to reduce $\mathrm{C}_{\mathrm{L}_{\mathrm{a}}}$.

As emphasized in Ref. 25, $M_{u}$ is an important design parameter which may destabilize the long-period longitudinal motion (shown there via root locus sensitivity analysis). Its numerical value is a function of the airplane configuration and of the specific flight condition. For example, a configuration design could feature a high wing and wingmounted engines and propellers. The resulting variations in aerodynamic forces acting through a larger moment arm may affect $M_{u}$ substantially. Another aerodynamic coefficient which may be affected by such a design is ${ }_{8 \mathrm{~T}}$.

With regard to the $\mathrm{Z}_{\mathbf{8 T}}$ derivative, negative values may correspond to augmented upper-surface-blowing (Thrust Vector Control or TVC), and positive values simulate an adverse situation in which the engine is tilted forward without an appropriate thrust compensation, resulting in airplane descent along with horizontal acceleration. If the design goal is a configuration with a shorter body, the vertical force sensitivity of the elevator $z_{\delta E}$ may be affected. To preserve its moment effectiveness $M_{\delta E^{\prime}}$ the designer may increase the elevator area and create more negative $\mathrm{Z}_{\delta \mathrm{E}}$. On 
the other hand, introduction of a canard control surface, as on Burt Ruttan's "Vari - Eze" or on the old Man's Aircraft Co.'s "OMAC l" aircraft, may result in $\mathrm{Z}_{\delta \mathrm{E}}>0$

It is important to evaluate the effect of variation of these aerodynamic coefficients under realistic SPIFR conditions as guidance for future designs. The ranges of variation in the chosen stability and control derivatives were defined with respect to the nominal Navion, which possesses "well-behaved" mid-spectrum dynamic characteristics (eq. $(2-50)$ to $(2-67))$.

Along with reflecting trends in GA aircraft design, these ranges of variation have to account for practical implementation issues. For example, aerodynamic coefficients such as $M_{u}, Z_{\delta T^{\prime}}$ and $M_{\delta T}$ have very small numerical values for the basic ARA configuration. For such parameters, instead of defining the range of variation in terms of a percentage of the nominal value, a different approach was taken. The variation envelopes were opened in preliminary flight-testing by increasing the absolute value of the coefficient to a level, which has a distinct effect on the aircraft's dynamic response. $\quad M_{\delta} \mathrm{T}$ is an example of a parameter for which it was important to obtain a significantly wide absolute range of variation, because of its sensitivity to vari- 
ations in trim conditions and, in particular, to the horizontal stabilator incidence setting. Note also that in flying the $\mathrm{Z}_{\delta \mathrm{T}}$ and the $\mathrm{M}_{\delta_{\mathrm{T}}}$ configurations, $\mathrm{X}_{\delta_{\mathrm{T}}}$ was kept constant. As this coefficient reflects the actual power setting, the physically more important variations in the ratios $\mathrm{Z}_{\delta \mathrm{T}} / \mathrm{X}_{\delta \mathrm{T}}$ and $\mathrm{M}_{\delta \mathrm{T}} / \mathrm{X}_{\delta \mathrm{T}}$ were simulated. The numerical values for the final SPIFR configurations, with respect to the nominal one (eq. (2-50) to $(2-67)$ ), are:

$$
\begin{aligned}
& x_{u}=0 ;-0.166 \mathrm{sec}^{-1}(2008 \text { of Nominal) } \\
& \Delta z_{u}= \pm 308 \text { of Nominal } \\
& \Delta z_{w}= \pm 508 \text { of Nominal } \\
& \Delta M_{u}= \pm 0.01 \mathrm{ft}^{-1}-\mathrm{sec}^{-1} \\
& z_{\delta_{E}}= \pm(2008 \text { of Nominal) } \\
& \Delta z_{\delta_{T}}= \pm 0.13\left(\mathrm{ft} / \mathrm{sec}^{2}\right) / \mathrm{mm} \\
& \Delta M_{\delta_{T}}= \pm 0.014\left(\mathrm{rad} / \mathrm{sec}^{2}\right) / \mathrm{mm}
\end{aligned}
$$

For briefness in the following discussion, a reference number was assigned to each configuration (Table 2.1).

The details of the derivation of the candidate criteria for these configurations are given in Appendix B. The re- 
TABLE 2.1

Reference Number Assignment to the SPIFR Configurations

\begin{tabular}{|c|c|c|c|c|c|c|c|c|c|c|c|c|c|c|c|c|}
\hline \multirow{2}{*}{$\begin{array}{l}\text { CON- } \\
\text { FIGU- } \\
\text { RATION }\end{array}$} & \multicolumn{2}{|c|}{$\Delta \mathrm{M}_{\delta \mathrm{T}}$} & \multicolumn{2}{|c|}{$\Delta \mathrm{z}_{\delta \mathrm{T}}$} & \multicolumn{2}{|c|}{$\mathrm{z}_{\delta \mathrm{E}}$} & \multicolumn{2}{|c|}{$\Delta \mathrm{M}_{\mathrm{u}}$} & \multicolumn{2}{|c|}{$\Delta \mathrm{z}_{\mathrm{u}}$} & \multicolumn{2}{|c|}{$\mathrm{x}_{\mathrm{u}}$} & \multicolumn{2}{|c|}{$\Delta \mathrm{z}_{\mathrm{w}}$} \\
\cline { 2 - 15 } & $>0$ & $<0$ & $>0$ & $<0$ & $2 \mathrm{~N}$ & $-2 \mathrm{~N}$ & $>0$ & $<0$ & $-0.3 \mathrm{~N}$ & $0.3 \mathrm{~N}$ & 0 & $2 \mathrm{~N}$ & $-0.5 \mathrm{~N}$ & $0.5 \mathrm{~N}$ \\
\hline CODE & 1 & 2 & 3 & 4 & 5 & 6 & 7 & 9 & 10 & 11 & 12 & 13 & 14 & 15 & 16 \\
\hline
\end{tabular}

sults of this derivation are presented in Table 2.2 in a format, compatible with the statistical analysis of Chapter 4. This analysis is to decide which candidate criteria materialize as actual flying qualities criteria, following realistic SPIFR mission flight testing. Figure 2.1 is a visualization of the range of variation of the $\left(\zeta \omega_{n}\right)_{p}$ candidate flying qualities criterion. 
TABLE 2.2

SPIFR Candidate Criteria

\begin{tabular}{|c|c|c|c|c|c|c|c|c|c|c|}
\hline \multirow[b]{2}{*}{$\begin{array}{l}\text { CONFIG- } \\
\text { URATION }\end{array}$} & \multirow[b]{2}{*}{$\begin{array}{l}\vdots_{\mathrm{RAD} / \mathrm{SEC}}^{\left(\zeta \omega_{n}\right)_{p}} \\
\operatorname{Ron}\end{array}$} & \multicolumn{3}{|c|}{$\begin{array}{l}\Delta \gamma_{\text {comm }}=4 \mathrm{deg} \\
\Delta v_{\text {comm }}=10 \mathrm{kt}\end{array}$} & \multirow[b]{2}{*}{$\begin{array}{r}\tau_{v}(\delta E) \\
\text { SEC }\end{array}$} & \multirow[b]{2}{*}{$\begin{array}{l}\tau_{v}(\delta T) \\
\operatorname{SEC}\end{array}$} & \multirow[b]{2}{*}{$\begin{array}{l}\tau_{\gamma}(\delta T) \\
\operatorname{SEC}\end{array}$} & \multirow[b]{2}{*}{$\begin{array}{l}A_{v}(\delta E) \\
\text { FPS }\end{array}$} & \multirow[b]{2}{*}{${ }_{\mathrm{DEG}}^{\mathrm{A}_{\gamma}(\delta E)}$} & \multirow[b]{2}{*}{$\begin{array}{l}A_{v}(\delta T) \\
\text { FPS }\end{array}$} \\
\hline & & $\begin{array}{l}\frac{\Delta \theta^{\star}}{\Delta \bar{V}_{\text {comm }}} \\
{[\mathrm{DEG} / \mathrm{KT}]}\end{array}$ & \begin{tabular}{|l|}
$\Delta \mathbf{f}_{\mathbf{s}^{*}}$ \\
$\Delta V$ comm \\
{$[\mathrm{LB} / \mathrm{DEG}]$} \\
\end{tabular} & $\begin{array}{l}\Delta \bar{f}_{\mathbf{g}}{ }^{\star} \\
\Delta \gamma_{\text {comm }} \\
{[\mathrm{LB} / \mathrm{DEG}]}\end{array}$ & & & & & & \\
\hline 1 & 0.054 & -4.7 & 4.7 & .17 & 4.5 & 0.50 & 6.0 & 3.3 & 2.5 & 3.3 \\
\hline 2 & 0.054 & -4.7 & 4.4 & 1.66 & 4.5 & 8.0 & 3.5 & 3.3 & 2.5 & 3.7 \\
\hline 3 & 0.054 & -4.7 & 5.0 & -1.38 & 4.5 & 2.0 & 8.0 & 3.3 & 2.5 & 5.0 \\
\hline 4 & 0.054 & -4.9 & 4.8 & -0.69 & 4.5 & 1.0 & 7.0 & 3.3 & 2.5 & 4.1 \\
\hline 5 & 0.054 & -4.6 & 4.5 & 0.83 & 4.5 & 9.0 & 4.5 & 3.3 & 2.5 & 3.0 \\
\hline 6 & 0.054 & -5.1 & 5.0 & .18 & 4.5 & 0.50 & 6.0 & 3.1 & 2.3 & 3.3 \\
\hline 7 & 0.054 & -4.1 & 3.9 & .14 & 4.5 & 0.50 & 6.0 & 3.9 & 2.9 & 3.3 \\
\hline 9 & 0.067 & -4.7 & 6.2 & .17 & 4.0 & 0.25 & 5.0 & 2.4 & 2.1 & 2.9 \\
\hline 10 & 0.040 & -4.6 & 3.2 & .17 & 5.5 & 0.50 & 7.0 & 5.0 & 3.1 & 3.9 \\
\hline 11 & 0.047 & -3.7 & 3.4 & .17 & 5.5 & 0.50 & 7.0 & 4.3 & 2.8 & 3.6 \\
\hline 12 & 0.060 & -5.7 & 5.9 & .17 & 4.0 & 0.25 & 5.0 & 2.6 & 2.2 & 3.0 \\
\hline 13 & 0.011 & -4.7 & 4.7 & .17 & 4.0 & 0.50 & 5.0 & 4.8 & 3.1 & 4.3 \\
\hline 14 & 0.096 & -4.7 & 4.7 & .17 & 5.0 & 0.50 & 7.0 & 2.2 & 1.9 & 2.8 \\
\hline 15 & 0.056 & -9.1 & 9.8 & .36 & 3.75 & 0.50 & 6.0 & 1.7 & 1.3 & 3.1 \\
\hline 16 & 0.052 & -3.4 & 3.2 & .11 & 5.0 & 0.50 & 6.0 & 4.7 & 3.4 & 3.5 \\
\hline
\end{tabular}


(m) - NUMBER OF COINCIDING POINTS

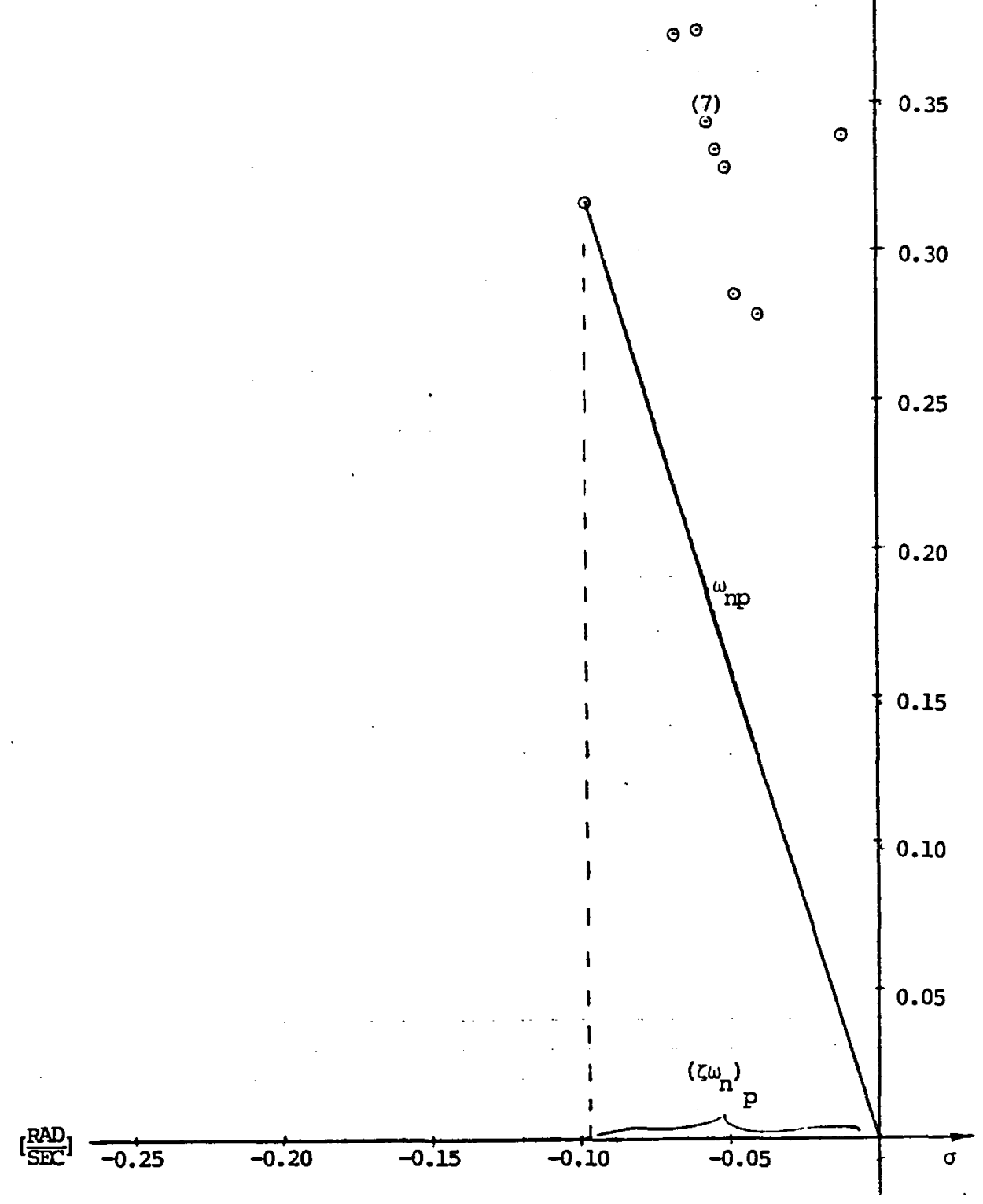

Figure 2.1: Total Damping Variations for SPIFR Configurations 


\subsection{IMPLEMENTATION OF SPIFR CONFIGURATIONS VIA IMPLICIT- MODEL-FOLLOWING ALGORITHM}

The chosen SPIFR configurations were simulated on the ARA using the Implicit-Model-Following algorithm to generate control laws (Ref. 27). State equations of the type of eq. (2-43) may be written for the nominal ARA configuration (subscript ARA) and for the configurations to be simulated ( subscript $M$ ),

$$
\begin{aligned}
& \underline{\dot{\mathrm{x}}}_{\mathrm{ARA}}=\mathrm{F}_{\mathrm{ARA}} \underline{\mathrm{x}}_{\mathrm{ARA}}+\mathrm{G}_{\mathrm{ARA}} \triangle_{\mathrm{ARA}} \\
& \dot{\Delta x}_{M}=F_{M} \underline{x}_{M}+G_{M} \underline{u}_{M}
\end{aligned}
$$

The objective was to obtain the control vector $\underline{u}_{A R A}$ ' which will make the ARA respond as the required configuration. The perfect model following objective is,

$$
\dot{\Delta x}_{A R A}=\dot{x}_{M}
$$

given $\quad \underline{x}_{A R A}(0)=\underline{x}_{M}(0)$

Substituting eq. $(2-88)$ and $(2-89)$ into eq. $(2-90)$ and rearranging, 


$$
\begin{aligned}
\underline{\Delta u}_{A R A} & =G_{A R A}^{\#}\left[\left(F_{M}-F_{A R A}\right) \underline{\Delta x}_{A R A}+G_{M} \underline{u}_{M}\right]= \\
& =c_{B} \underline{\Delta x}_{A R A}+c_{F} \underline{u}_{M}
\end{aligned}
$$

where

$$
G_{A R A}^{\#} \quad=\quad\left(G_{A R A}^{T} G_{A R A}\right)^{-1} G_{A R A}^{T}
$$

Eq. $(2-90)$ renders,

$$
\triangle \underline{x}_{\mathrm{ARA}} \quad=\quad \underline{\Delta x}_{\mathrm{M}}
$$

Thus, $\triangle \underline{x}_{A R A}$ is the solution of eq. (2-89). Erzberger has shown that this yields perfect model following if there are no additional constraints on control usage or parameter insensitivity. A block diagram of the derived algorithm is presented in Figure 2.2.

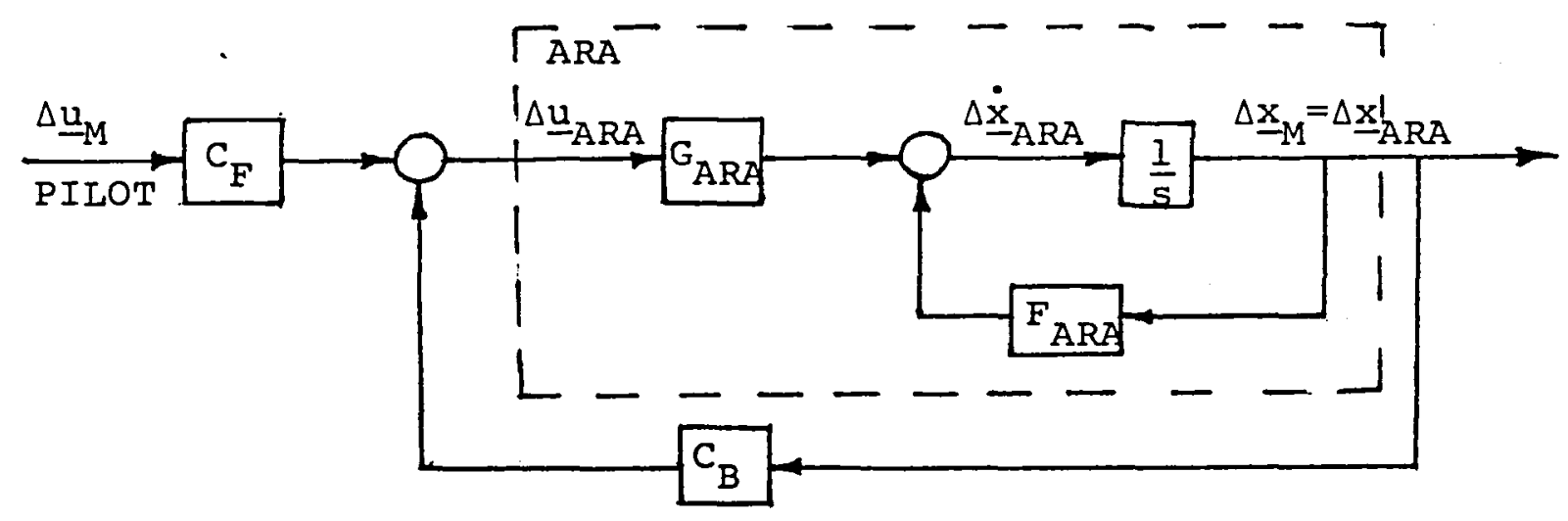

Figure 2.2: Block Diagram for Implicit Model-Following on the ARA 
The elements of $C_{B}$ and of $C_{F}$ constitute the theoretical set of gains required to simulate a given configuration in flight, using the ARA. However, to implement this set of gains, we must account for calibration constants, control gearing ratios, and, in some instances, for physical relationships between the sensor output and the associated state variables, which are fed back via $C_{B}$. These effects are now incorporated into the scheme of Fig. 2.2 (Fig. 2.3).

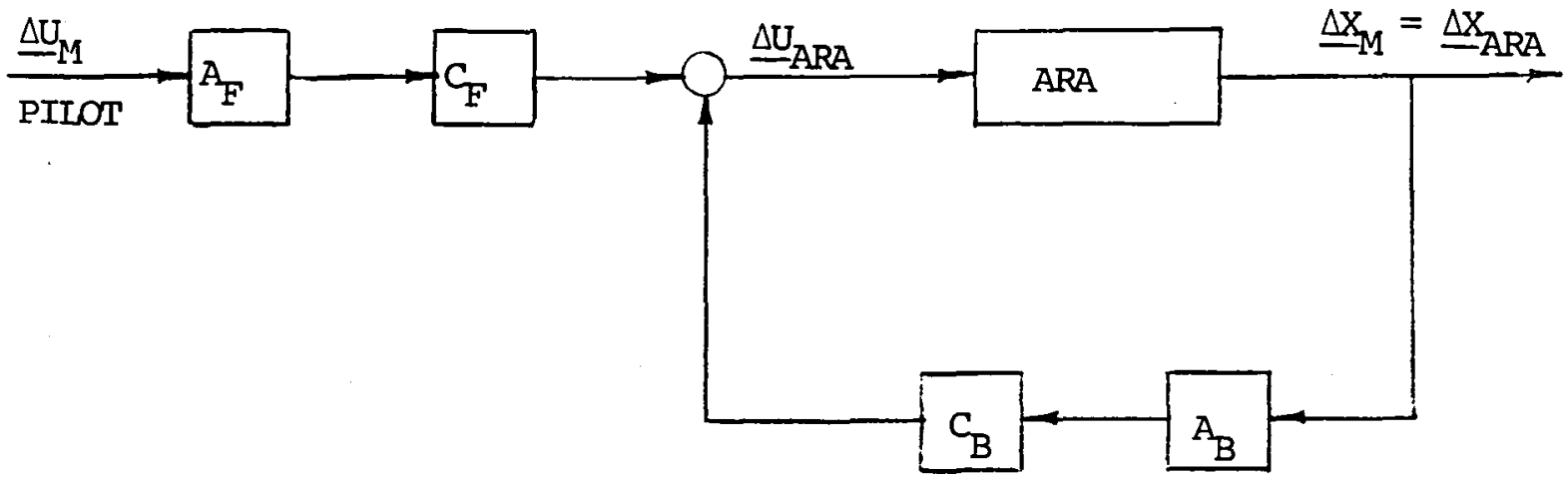

Figure 2.3: Implementation of a SPIFR Configuration on the In-Flight Simulator

The added matrices $A_{F}$ and $A_{B}$ provide the required engineering corrections to the theoretical gain matrices $C_{F}$ and 
$\mathrm{C}_{\mathrm{B}^{\prime}}$ respectively. They include the calibration constants (slopes and biases), which translate these gains into actual potentiometer settings. This information has been compiled in the "VRA and ARA Calibration Data Manual" (Ref. 28). AF also accounts for the gearing ratios between the cockpit controls and the control effectors. ${ }^{2}$

As already mentioned, in some cases the required inputs to the feedback loop in the scheme of Fig. 2.3 are more complex functions of the corresponding sensor output than a trivial one-to-one equivalence. For example, the angle of attack sensor, although positioned as far as was practically possible from the wing tip, is affected by "upwash" in the flow. The relationships between the measured values and the actual physical variables were incorporated into matrix $A_{B}$ ' making it, in a sense, a static estimator.

Finally, the actual potentiometer setting matrices for the feedforward and the feedback loops are $\left[C_{F} A_{F}\right]$ and $\left[C_{B} A_{B}\right]$,

$$
\underline{\Delta a}_{A R}=\left[C_{F} A_{F} \underline{\Delta u}_{M}+\left[C_{B} A_{B}\right] \underline{\Delta x}_{A R A}\right.
$$

2 The control effectors are the actual mechanical devices (elevator, throttle, etc.), which apply the aerodynamic forces and torques which move the airplane. 
The elements of the potentiometer setting matrices constitute a direct output of the implicit model following computer program. The program was run for all SPIFR configurations, and the results are summarized in Table 2.3. Only the primary gain setting for each configuration is presented, as the secondary cross effects turned out to be negligible. 
TABLE 2.3

Gain Settings for In-Flight Simulation of SPIFR
Configurations

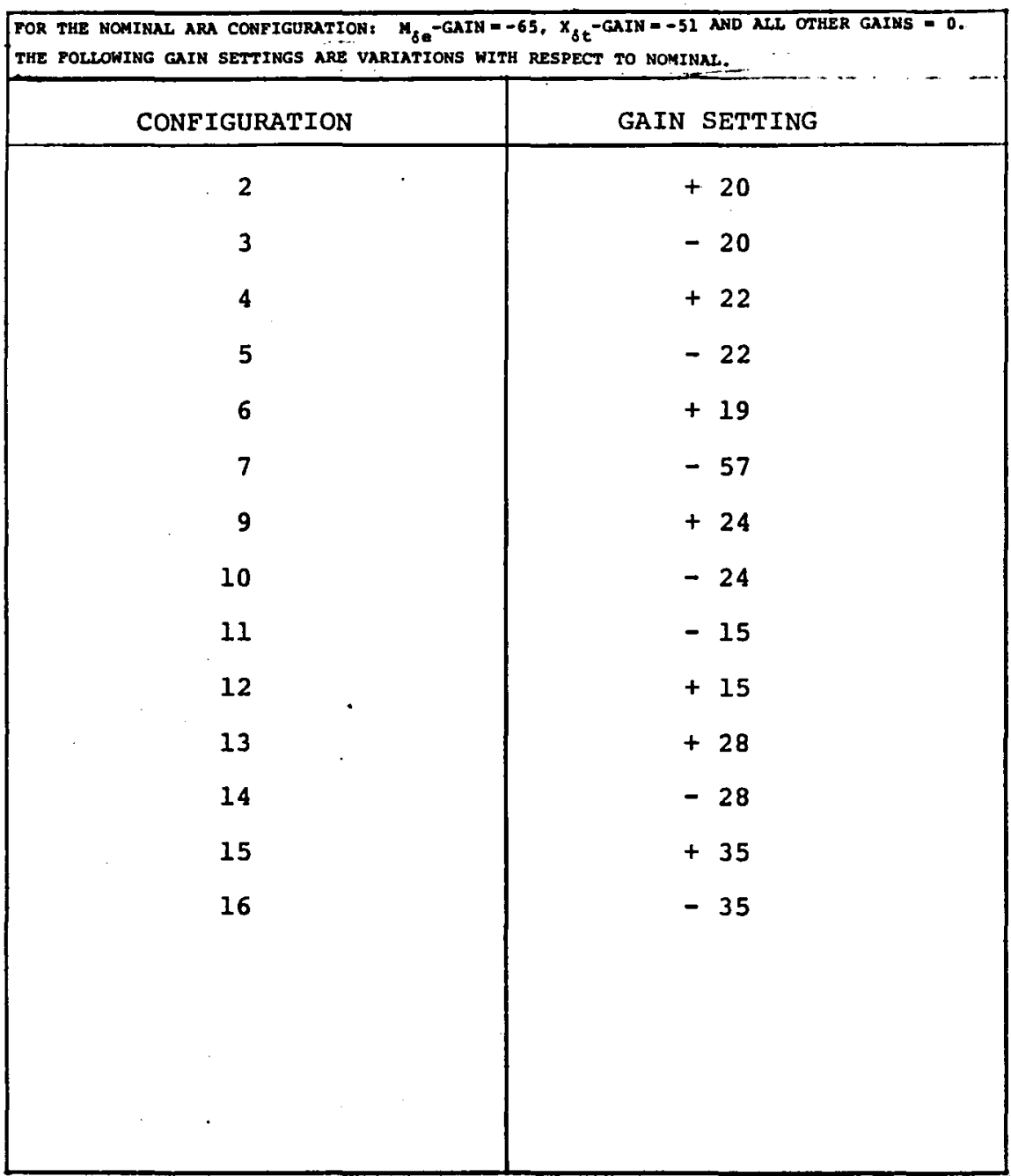


2.4 EFFECTS OF NAVIGATIONAL ACCURACY AND OF THE "LEARNING CURVE" ON MISSION PLANNI

For realistic SPIFR simulation, the mission has to contain several typical flight-path segments, including

* Climb, acceleration, and cruise with airspeed retrimming

* Holding pattern

* Deceleration and descent

* Interception of the landing system beam

* Approach and missed-approach go-around

Also, a realistic $\operatorname{VOR}^{3}$ navigation simulation should consist of engaging navigational stations in the "TO" as well as the "FROM" mode. The above considerations roughly size the SPIFR mission simulation to a flight duration of about thirty minutes, with the geometry shown in Fig. 2.4.

One problem associated with selecting the flight path geometry is the "learning curve" effect. This is the ability of a human being to improve his performance by repeating the same task. Flying all missions along the same trajectory allows the pilot to memorize control patterns, reducing the navigation workload to a level that is unrealistic for a real mission. To cope with this issue, additional flight path variants were devised (Fig. 2.5, 2.6, 2.7). All vari-

3 VOR is a navigational system and the acronym stands for Very-high-frequency Omni Range. 
ants have different altitude and airspeed profiles but, are of comparable structure and flight duration. Also, the order in which the various configurations and tracks were flown was randomized.

The other problem associated with the selection of flight path geometry is navigational accuracy. Appropriate trajectory planning may keep the flight technical error to minimum and prevent divergence between navigational tracks. It may also improve the accuracy of post-flight flight path reconstruction, as will be shown in Chapter 3. The standard navigational modes for GA flight are VOR/VOR, and VOR/DME. At least two VOR-only stations are required to achieve a horizontal "fix" of the aircraft's position (a single VOR/DME station is sufficient). With proper geometry these modes can provide an accurate position estimate.

Geometric Dilution of Precision (GDOP) affects navigational accuracy. GDOP is an inaccuracy due to the nonperpendicularity of the lines connecting the aircraft with the engaged stations. Applying analytical geometry to the typical situation depicted in Fig. 2.8 and assuming that the two navigation stations' errors are statistically uncorrelated,

$$
\sigma=(1 / s \theta)\left[\sigma_{1}^{2}+\sigma_{2}^{2}\right] 1 / 2
$$




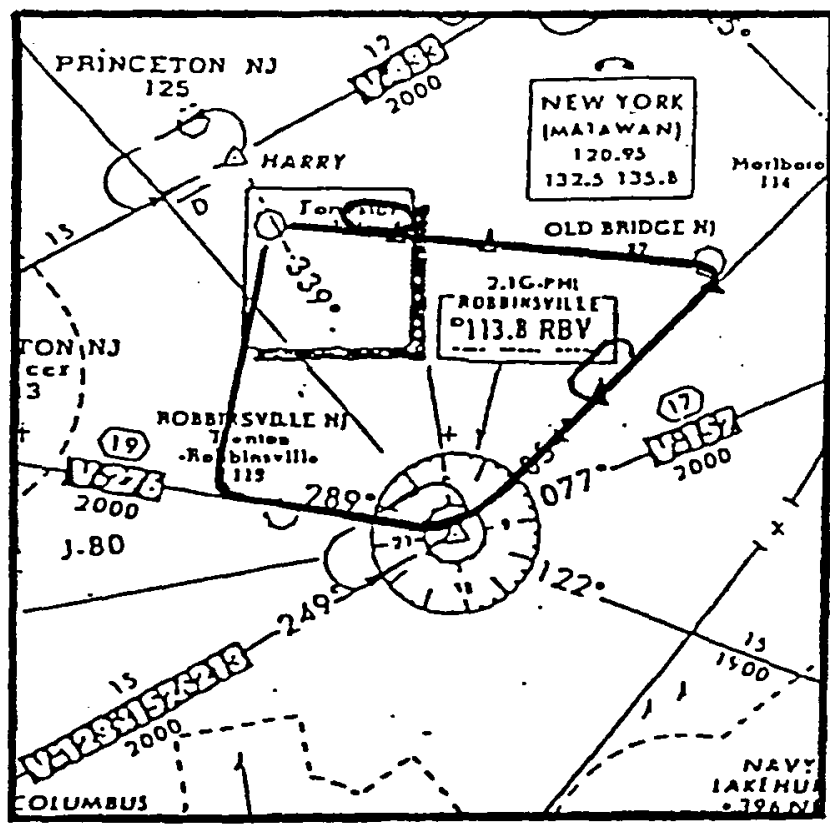

Figure 2.4: Variant I.

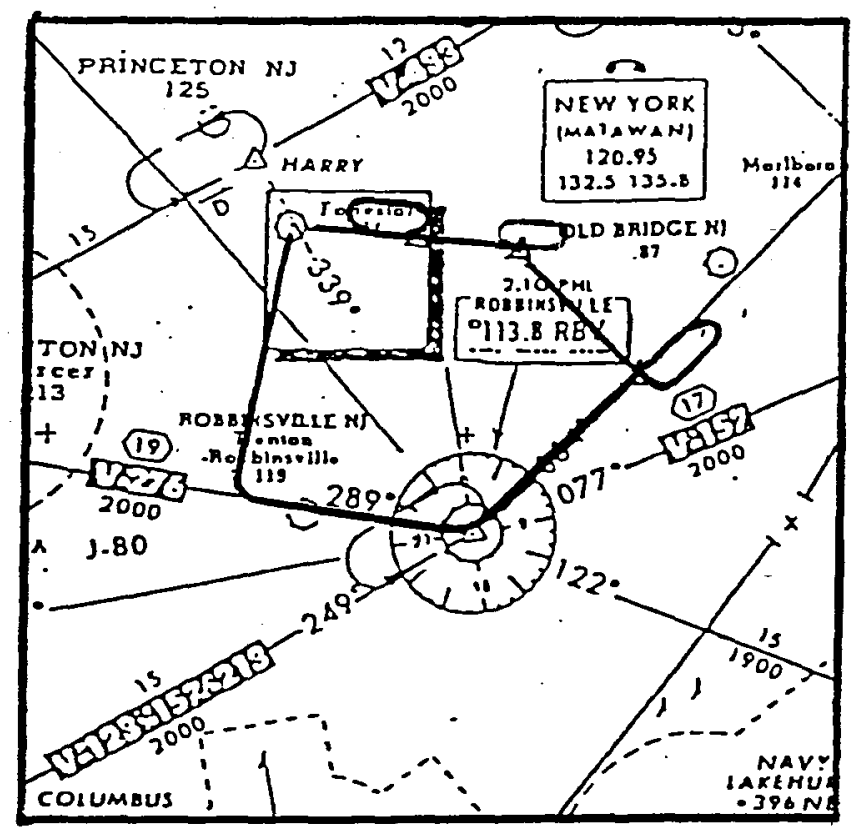

Figure 2.5: Variant II.

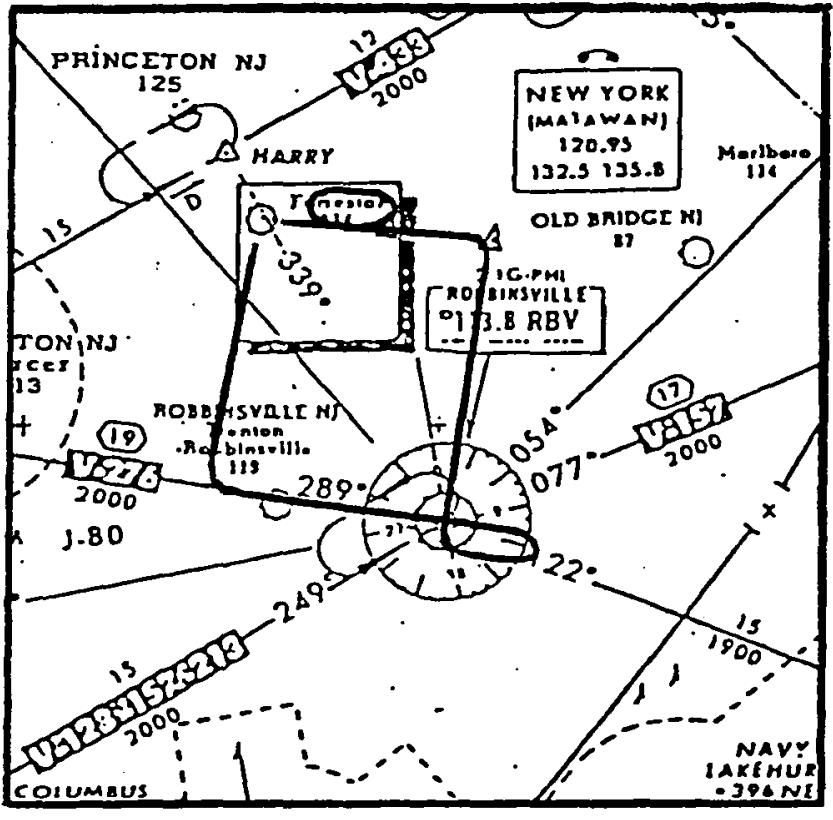

Figure 2.6: Variant III.

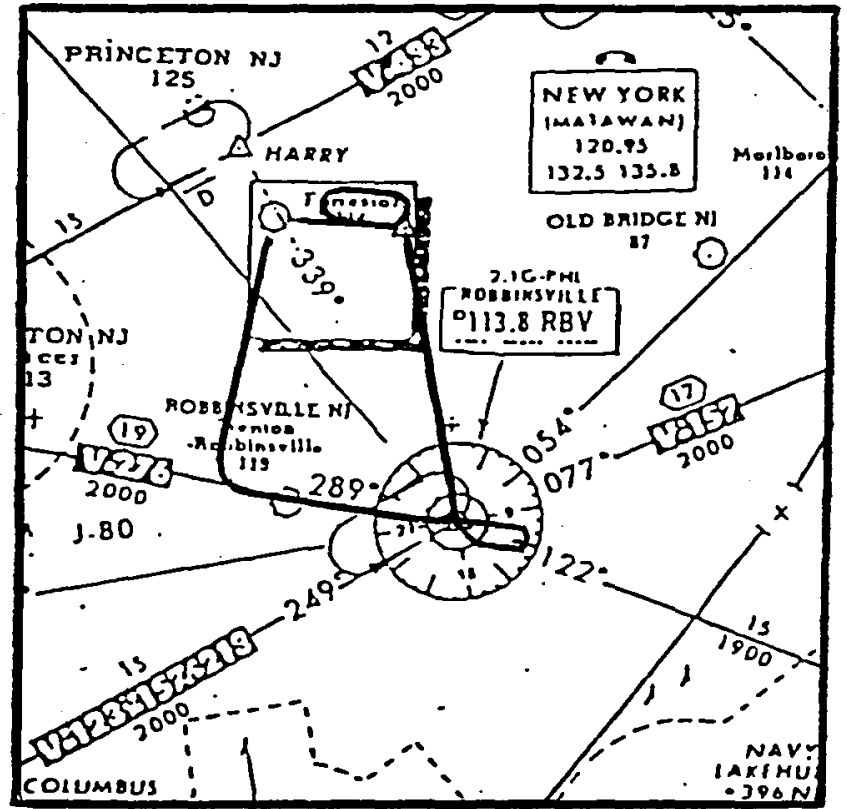

Figure 2.7: Variant IV.

Figures 2.4 to 2.7 SPIFR Flight Path Variants. 


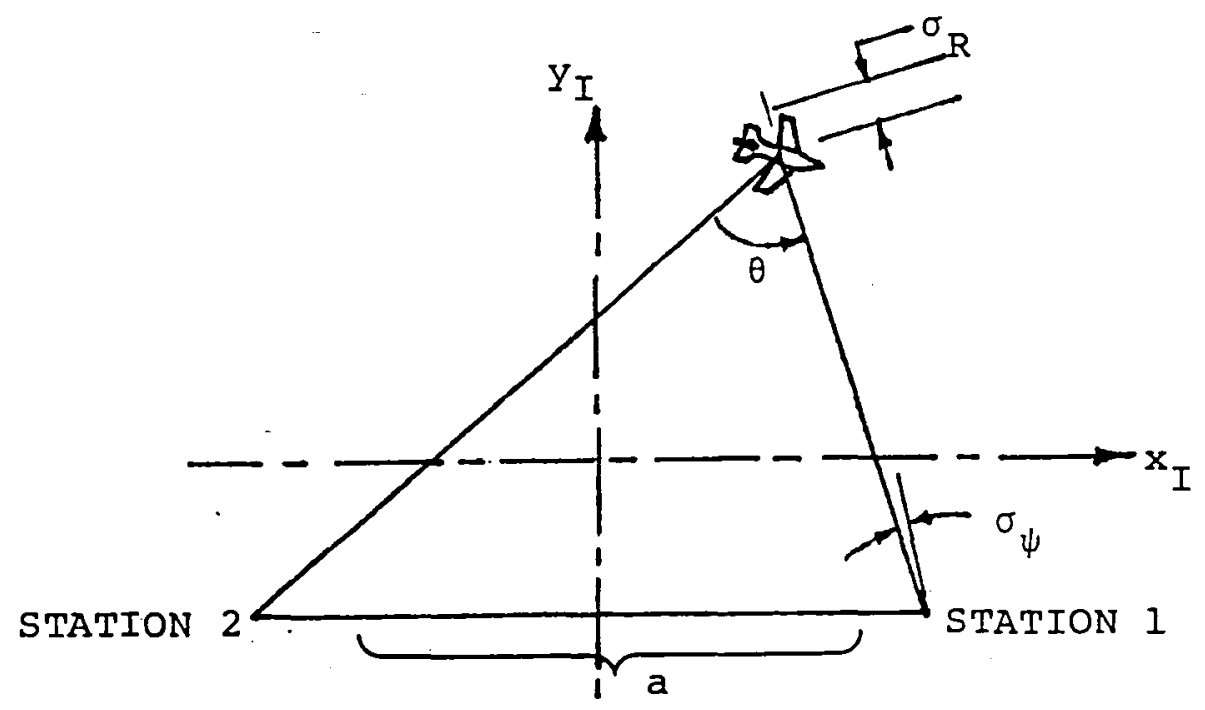

Figure 2.8: Ground Stations Engagement in the VoR/Vor or the DME/DME Modes.

The $1 / \mathrm{s} \theta$ term reflects the GDOP effect. For angles between radials in the vicinity of $\theta=0$ or $\theta=180 \mathrm{deg}$, the position error becomes very large, becoming infinite in the limit. To improve position accuracy using two similar ground stations while flying a given leg, it is desirable that the stations be as nearly perpendicular as possible. For VOR/VOR, eq. (2-95) can be rewritten as,

$$
\mathrm{VOR} / \mathrm{VOR}=\frac{a \sigma_{\psi}}{\sqrt{2} \mathrm{~s}^{2} \theta}\left(1+\mathrm{s}^{2} \theta\right)^{1 / 2}
$$

For DME/DME, eq. (2-95) becomes,

$$
\mathrm{DME} / \mathrm{DME}=\sqrt{2} \sigma_{\mathrm{R}} / \mathrm{s} \theta
$$


The accuracy requirements imposed by the FAA are (Ref. 29) :

$$
\left.\begin{array}{rl}
\sigma_{\psi} \equiv \sigma_{\text {VOR }}=1.9^{\circ} \\
\sigma_{\mathrm{R}}=\sigma_{\mathrm{DME}}= & 0.15 \% \text { range or } 0.1 \text { mile: } \\
& \text { whichever is larger }
\end{array}\right\}
$$

Numerical values of eq. $(2-98)$ and dependence of $\sigma_{\text {VoR/VoR }}$ on $\sin ^{-2} \theta$ suggest that this navigation mode is much less accurate than the DME/DME mode. For example, at a range of 50 miles from both stations and for $\theta=30 \mathrm{deg}$ the VOR/VOR error is 2.71 miles, while the DME/DME error is 0.28 miles; the results favor the DME/DME pairing at greater ranges. Based on this observation and on the feasibility of microprocessor-controlled sequential engagements of several DME stations, it was decided that a DME switching technique should be used to improve the flight path reconstruction accuracy. In particular, this accuracy improvement may be achieved by making use of redundant measurements, while applying the optimal Kalman filtering/smoothing algorithm. 


\subsection{IN=FLIGHT VERIFICATION OF THE SPIFR RESEARCH CONCEPT}

To test the complete SPIFR-mission-simulation concept, a series of preliminary flights were carried out. Its main objectives were to verify the realism of simulation of the SPIFR regime environment and the in-flight configuration matching capability. After extensive hangar and in-flight checks of the aircraft system modifications, of the new navigation/comunication package and of the onboard experimental setup, the proposed instrument tracks (Fig. 2.4 to 2.7 ) were flown, totalling about ten flight hours.

These preliminary flights have shown that the tasks appear to simulate IFR missions, which are realistic in both geometry (Section 2.4) and workload. With regard to workload, the McGuire Air Force Base approach control frequency was tuned in for realistic background chatter. The safety pilot, who played the role of the air traffic controller, delivered real-time clearances to the evaluation pilot. Clearances were delivered and read back, after being copied, using the voice-operated intercom. To avoid interference, the McGuire approach control audio was deselected during these communications. Tuning in VOR/DME stations, VOR tracking and navigation to waypoints, executing holding pattern turns, leveling off from climb, airspeed retrimming, changing altitude, localizer interception, and other ele- 
ments of a typical SPIFR scenario were excersized during these flights. The first of the four navigational tracks of Fig. 2.4 to 2.7 was found to take about 208 more time than the other three. To keep the missions comparable in workload, it was not flown during the actual flight test series.

To evaluate successfully the relative importance of the low-frequency airframe dynamic response and the SPIFR navigation/communications tasking, all other workloading effects had to be minimized. Thus, all the test flights of the program were carried out under zero-to-light natural turbulence conditions. Turbulence, which is a higher-frequency phenomenon, could have obscured the long-period configuration trends.

The preliminary flight tests also were used to verify various in-flight procedures. Normally three SPIFR missions were flown per flight. Before a mission was initiated, the safety pilot had to "dial in" the aerodynamic configuration gains (Table 2.3 ). Then he turned the "new" aircraft over to the evaluation pilot for a brief familiarization period. During this period the safety pilot switched digital recording cartridges, brought the data acquisition system into the "stand by" mode, and tuned in the McGuire Air Force Base approach control radio frequency. 
To confirm the matching capability, each of the chosen aerodynamic configurations was simulated in flight during these preliminary experiments. As shown in Section 2.2, the interactive approach which combined the theoretical considerations with real-life flight testing, played an important role in the program's success.

To verify the ARA dynamic model, through which the SPIFR candidate flying qualities criteria were derived, numerical values of several of them were obtained experimentally and compared to the preliminary calculations. The phugoid natural frequency and damping characteristics were measured by first stabilizing the airplane and trimming the elevator control force to zero at 2000-ft altitude and 75-kt airspeed. The airspeed then was decreased by about 10 to 20 knots using the elevator control. At this point the yoke control was smoothly returned to its zero position. The resulting phugoid oscillation was permitted to persist for about 3 to 4 cycles. This procedure was carried out for configurations No. 1 and 9 to 14 which, as may be observed from Table 2.2, were quite different from the nominal Navion characteristics (configurations No. 2 to 7 consist of control effect changes, which do not affect the phugoid eigenvalue). After the damping of the short-period motion, the phugoid is characterized as follows, 


$$
\begin{aligned}
& \alpha \cong \text { const. } \\
& \gamma \cong \theta
\end{aligned}
$$

From the recorded $\theta(t)$ time histories the time to half the amplitude, $t_{1 / 2}$, and the period of the oscillation, $P$ were obtained. Then, using the technique of Ref. 30 ,

$$
\begin{aligned}
& \operatorname{Im}_{\lambda}=2 \pi / \mathrm{P}=\omega_{\mathrm{np}}\left(1-\zeta_{\mathrm{p}}^{2}\right)^{1 / 2} \\
& \operatorname{Re}_{\lambda}=1 \mathrm{n} 2 / t_{1 / 2}=\omega_{\mathrm{np}} \zeta_{\mathrm{p}}
\end{aligned}
$$

The resulting values of $\omega_{n p}$ and $\zeta_{p}$ were computed using eq. $(2-47)$ to $(2-49)$, and crosschecked with the theoretical values (Table B.l). For another confirmation of the theoretical results, numerical values of $\Delta \theta^{*}, \Delta \delta_{E}^{*}$ and of $\Delta \delta_{T}^{*}$ for $\Delta \mathrm{v}_{\text {comm }}=10 \mathrm{kt}$ were obtained through flight testing and were compared to the corresponding values in Table 2.2. For each of the fifteen configurations, the airplane was trimmed at 1500-ft altitude and 75-kt airspeed, then retrimmed at the same altitude and $85 \mathrm{kt}$. Examples of the comparison between theoretical prediction, and experiment are given in Table 2.4. The phugoid natural frequency, $\omega_{n p}$ results are somewhat biased down with respect to the predicted values, but the trends are correct. For the pitch angle change, $\Delta \theta^{*}$, the effects of variation of the aerodynamic coeffi- 
cients were also obtained to a good approximation. This comparison renders confidence in the theoretically-derived candidate flying qualities criteria.

TABLE 2.4

Experimental Verification of the Theoretical Dynamic Model

\begin{tabular}{|c|c|c|c|c|}
\hline \multirow{2}{*}{ CONFIGURATION } & \multicolumn{2}{|c|}{$\omega_{n p}[R A D / S E C]$} & \multicolumn{2}{|c|}{$\Delta \theta *[D E G]$ FOR $\Delta V_{C O M M}=10 \mathrm{Km}$} \\
\hline & PREDICTED & EXPERIMENTAL & PREDICTED & EXPERIMENTAL \\
\hline 1 & 0.34 & 0.32 & -4.7 & -4.4 \\
\hline 2 & & & -4.7 & -4.3 \\
\hline 3 & & & -4.7 & -4.2 \\
\hline 4 & & & -4.9 & -4.7 \\
\hline 5 & & & -4.6 & -4.2 \\
\hline 6 & & & -5.1 & -5.0 \\
\hline 7 & & & -4.1 & -3.9 \\
\hline 9 & 0.38 & 0.35 & -4.7 & -4.3 \\
\hline 10 & 0.28 & 0.26 & -4.6 & -4.7 \\
\hline 11 & 0.29 & 0.27 & -3.7 & -3.4 \\
\hline 12 & 0.38 & 0.36 & -5.7 & -5.5 \\
\hline 13 & 0.34 & 0.33 & -4.7 & -4.4 \\
\hline 14 & 0.33 & 0.30 & -4.7 & -4.5 \\
\hline 15 & 0.35 & 0.34 & -9.1 & -8.2 \\
\hline 16 & 0.33 & 0.34 & -3.4 & -3.6 \\
\hline
\end{tabular}




\section{Chapter 3 \\ FLIGHT PATH RECONSTRUCTION - THEORY AND APPLICATION}

To decide which of the candidate criteria (Table 2.2) are suitable metrics of GA SPIFR flying qualities, a statistical comparison with experimentally derived system performance measures must be made. This required accurate estimates of flight path variables, which were measured directly or had to be reconstructed from other noisy measurements. First, Section 3.1 elaborates on the extensions to the system's mathematical model, that are required for flight path reconstruction. Section 3.2 discusses the post-flight optimal filtering and smoothing algorithms' formulations. Development of the filter tuning methodology and results of applying the algorithm to SPIFR experimental data are presented in Section 3.3 .

\subsection{DYNAMIC MODEL GENERALIZATION}

As pointed out in section 2.1, the system model should be generalized to allow accurate post-flight trajectory estimation. This generalization includes an introduction of a new Earth-centered Cartesian axis system, and it accounts for 
the effects of Earth rotation and geometry. This was done by defining the motion of the LLLN Cartesian coordinate frame with respect to this new axis system. The mathematical model of Section 2.1 will be revised, and the required modifications will be introduced wherever they are due. A closer look at this model reveals that it consists of two distinct submodels, the angular submodel (eq. (2-9) and (2-10)) and the translational submodel (eq. (2-2) and (2-11) plus auxiliaries). Such a division into lower-order submodels increases the numerical robustness of optimal smoothing. First, it is shown that it is compatible with the SPIFR instrumentation system (described in detail in Appen$\operatorname{dix} A)$

Rewriting the equations (2-9) and (2-10),

$$
\left[\begin{array}{l}
\dot{\phi} \\
\dot{\theta} \\
\dot{\psi}
\end{array}\right]=\mathrm{L}_{\mathrm{B}}^{\mathrm{I}}\left[\begin{array}{l}
\mathrm{p} \\
\mathrm{q} \\
\mathrm{I}
\end{array}\right]
$$

where

$$
I_{B}^{I} \triangleq \frac{1}{c \theta}\left[\begin{array}{ccc}
c \theta & s \theta s \phi & s \theta c \phi \\
0 & c \theta c \phi & -c \theta s \phi \\
0 & s \phi & c \phi
\end{array}\right]
$$


The SPIFR experimental setup provides measurements of both the angular rates $(p, q, r)$ and the Euler angles $(\phi, \theta$, 4). As will be shown, this information plus the model in eq. (3-1) and (3-2) can be used to obtain optimally smoothed estimates of the angular states. These accurate estimates may then serve as inputs into the translational submodel.

The "flat Earth" formulation of the translational submodel, which is given with respect to the LLLN coordinate frame in section 2.1 , is widely used in aircraft dynamics simulations and analyses. For navigation problems, a three-dimensional state equation eq. (2-11), describing inertial velocity usually is employed:

$$
\dot{x}_{I}=\underline{v}_{\text {air I }}+\underline{w}_{I}
$$

The inertial platform mechanization is supposed to take care of the dynamic effects associated with Earth rotation and to provide continuously LLLN-referenced inertial acceleration measurements $\underline{a}_{I} \cdot$ Ideally,

$$
\dot{\mathrm{v}}_{\mathrm{air} I}=\underline{\mathrm{a}}_{\mathrm{I}}+\underline{g}_{I}
$$

Corrections to position estimation $\underline{x}_{I}$ due to the kinematics of Earth rotation and geometry are then introduced algebraically, following the optimal filtering, which operates on differential error equations. Other navigational applications reconstruct position based on eq. (3-3), using "dead 
reckoning" information and on distance measurements (Ref. $31)$

The outputs of the SPIFR experimental system include three-axes angular attitudes and rates, strapdown body accelerations, aerodynamic angles, airspeed, altitude and distances to navigational stations; this constitutes a selfcontained body of information. As shown later in this chapter, appropriate processing of this information renders accurate estimates of flight path variables, yielding insight into both dynamic and navigational effects.

In order to achieve these objectives, the translational submodel must be generalized to include the effects of Earth rotation and geometry. Following Ref. 21, a set of spherical coordinates is defined (Fig. 3.1) with respect to the Cartesian set of axes, whose origin is the center of the sphere; $x_{E C^{-}}$and $z_{E C^{-}}$axes are in the $0 / 180$ deg-meridianplane with $z_{E C}$ pointing upwards and $x_{E C}$ pointing towards the zero meridian. The Cartesian set is rotating about $\mathbf{z}_{\mathrm{EC}}$ with $\boldsymbol{\Omega}=0.728 * 10^{-4} \mathrm{rad} / \mathrm{sec}$. The spherical coordinates are the latitude $\lambda$, the longitude $\mu$, and the aircraft distance from the center of the sphere, $R$.

Based on the above definitions, a relationship between the LLLN Cartesian and the spherical coordinates may be deduced, 


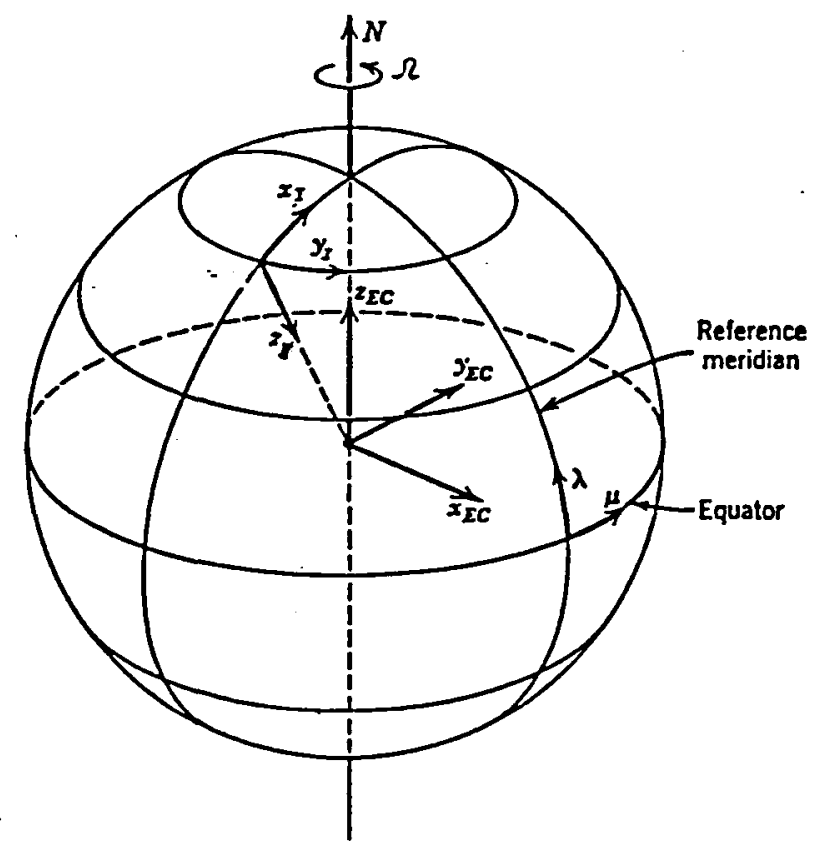

Figure 3.1: Earth Axes

$$
\left[\begin{array}{c}
\dot{x}_{I} \\
\dot{y}_{I} \\
\dot{z}_{I}
\end{array}\right]=\left[\begin{array}{c}
\dot{\lambda R} \\
\dot{\mu R c} \lambda \\
-\dot{R}
\end{array}\right]
$$

Substituting eq. $(3-5)$ into eq. (2-11),

$$
\left[\begin{array}{c}
\dot{\lambda} \mathrm{R} \\
\dot{\mu R c \lambda} \\
\dot{-R}
\end{array}\right]=\mathrm{H}_{\mathrm{B}} \underline{\underline{V}}_{\mathrm{air}}+\underline{\mathrm{W}}_{\mathrm{I}}
$$


with the right-hand-side of eq. (3-6) being a vector, whose physical interpretation remains in the LLLN Cartesian coordinate frame. The starting point for the derivation of the relationship equivalent to eq. (2-2) is (Ref. 2l),

$$
\begin{aligned}
& \underline{a}_{c B}=d / d t\left(\underline{v}_{a i r}+H_{I}^{B} \underline{W}_{I}\right)+\left(-\tilde{\omega}-\tilde{\omega}_{E}\right)\left(\underline{v}_{a i r}+H_{I}^{B_{W}} \underline{W}_{I}\right) \\
& +\operatorname{RH}_{I}^{\mathrm{B}} \Omega^{2}\left[\begin{array}{c}
s \lambda c \lambda \\
0 \\
c^{2} \lambda
\end{array}\right] \quad-\mathrm{H}_{\mathrm{I}}^{\mathrm{B}} \mathrm{g}_{I}
\end{aligned}
$$

where

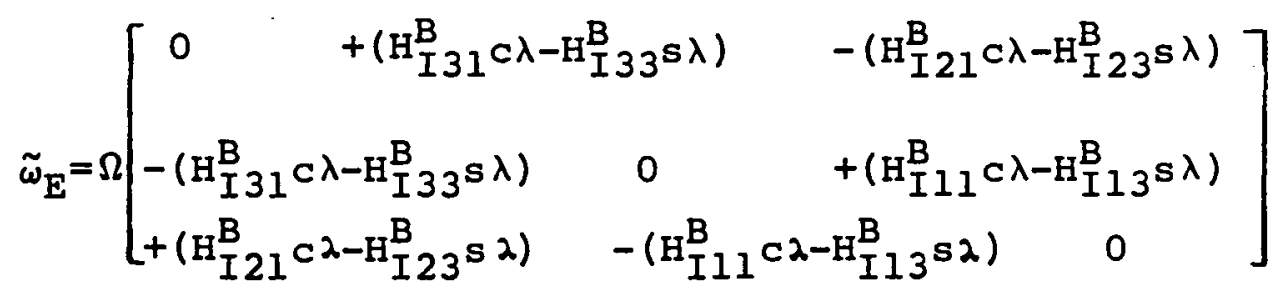

and all other components as defined in Section 2.1. Implementing the kinematic relationship,

$$
\dot{\mathrm{H}}_{\mathrm{I}}^{\mathrm{B}}=-\widetilde{\omega}_{\mathrm{I}}^{\mathrm{B}}
$$

eq. (3-7) may be cast into the format of eq. (2-2) plus a set of "correction" terms,

$$
\begin{aligned}
& \dot{\mathrm{v}}_{\mathrm{air}}=\underline{\mathrm{a}}_{\mathrm{B}}+\tilde{\tilde{\omega}} \underline{\mathrm{aij}}_{\mathrm{a}}+\mathrm{H}_{\mathrm{I}}^{\mathrm{B}} \underline{\mathrm{I}}_{\mathrm{I}}+\dot{?} \\
& +\tilde{\omega}_{E} \underline{V}_{\text {air }}-\Omega^{2} \mathrm{RH}_{I}^{\mathrm{B}}\left[\begin{array}{c}
s \lambda c \lambda \\
0 \\
c^{2} \lambda
\end{array}\right]+\left(2 \tilde{\omega}+\tilde{\omega}_{\mathrm{E}}\right) \mathrm{H}_{\mathrm{I}}^{\mathrm{B}} \underline{\mathrm{W}}_{\mathrm{I}}(3-10)
\end{aligned}
$$


Thus the generalized formulation of the translational submodel consists of eq. $(3-6)$ and $(3-10)$. Note that the strapdown accelerometers measure directly $\left(\underline{a}_{B}+H_{I}^{B} g_{I}\right)$.

To avoid numerical difficulties and retain physical insight it is instructive to cast eq. $(3-6)$ into the format of eq. (2-11), following the example of eq. (3-10). Define navigational-type state variables,

$$
\left.\begin{array}{l}
x_{c} \triangleq a \lambda \\
y_{c} \triangleq a \mu \cos \left(\lambda_{\circ}+\lambda\right) \\
z_{c} \triangleq z_{I}
\end{array}\right\}
$$

where $a=2.094 \times 10^{7} \mathrm{ft}$ is the semi-major-axis of the equator ellipse, $\lambda_{0}$ is the latitude at which the test-flight begins, implying zero initial conditions at this point for $x_{c}$ and $y_{c}$. The quantities $x_{c}$ and $y_{c} / \cos \left(\lambda_{o}+x_{c} / a\right)$ are the navigational coordinates $\lambda$ and $\mu$ with respect to the starting point $\left(\lambda_{0}, \mu_{0}\right)$, scaled by the constant a. The definition in eq. (3-11) has direct bearing on the flight-test methodology, as $\lambda_{0}$ and $\mu_{0}$ are inputs to the model and have to be known apriori. This constitutes no limitation, as the mission may be initiated over a navigational "fix" such as an airport reference point or a VOR/DME ground station. In order to substitute eq. $(3-11)$ into eq. (3-6), an expression relating $z_{I}$ and $R$ is required. Implementing the concept of the best-fitting sphere to the Earth surface at any point (Ref. 32), 


$$
R=-z_{I}+\rho_{G}
$$

where $P_{G}$ is the Gaussian radius of curvature,

$\rho_{G}=a\left[1-0.5 \epsilon^{2} \cos 2\left(\lambda_{0}+\lambda\right)\right]$

with $\epsilon^{2}=0.0067$ being the eccentricity of the equator ellipse.

Substitution of equations $(3-11)$ to $(3-13)$ into eq. $(3-6)$ renders,

$$
\begin{aligned}
\underline{\underline{x}}_{c}= & H_{B}^{I_{\text {Vir }}}+\underline{W}_{I}+\vdots \\
& +H_{C}\left(H_{B}^{I} \underline{V}_{a i r}+\underline{W}_{I}\right)
\end{aligned}
$$

where

$$
\mathrm{H}_{C}=\left[\begin{array}{ccc}
\gamma /{ }_{I-\gamma} & 0 & 0 \\
-y_{C} \tan \left(\lambda_{0}+\lambda\right) / a(1-\gamma) & \gamma /{ }_{I-\gamma} & 0 \\
\epsilon^{2} \sin 2\left(\lambda_{0}+\lambda\right) / I-\gamma & 0 & 0
\end{array}\right]
$$

with

$$
\gamma=1-\left(-z_{c}+p_{G}\right) / a
$$

The associated distance measurement equation is,

$$
r_{s i}=\left(x_{s i}^{2}+y_{s i}^{2}+z_{s i}^{2}\right)^{1 / 2}
$$

where 


$$
\left.\begin{array}{lr}
x_{s i}=a(1-\gamma) \cos \left(\lambda_{o}+\lambda\right) \cos \left(\mu_{\circ}+\mu\right)-\left(-z_{c}+\rho_{G}\right)_{s i} c \lambda_{s i} c \mu_{s i} \\
y_{s i}=a(1-\gamma) \cos \left(\lambda_{o}+\lambda\right) \sin \left(\mu_{0}+\mu\right)-\left(-z_{c}+\rho_{G}\right)_{s i} c \lambda_{s i} s \mu_{s i} \\
z_{s i}=a(1-\gamma) \sin \left(\lambda_{o}+\lambda\right) & -\left(-z_{c}+\rho_{G}\right)_{s i} s \lambda_{s i}
\end{array}\right\}
$$

with subscript si referring to the engaged DME stations.

An important observation may be made comparing the generalized translational submodel with the "flat Earth" approximation. Rewriting eq. $(3-10)$ and $(3-14)$,

$$
\begin{aligned}
& \underline{\dot{x}}_{\mathrm{C}}=\mathrm{H}_{\mathrm{B}}^{\mathrm{I}} \underline{\mathrm{V}}_{\mathrm{air}}+\underline{\mathrm{W}}_{\mathrm{I}}+ \\
& +\mathrm{H}_{\mathrm{C}}\left(\mathrm{H}_{\mathrm{B}}^{\mathrm{I}} \underline{\mathrm{V}}_{\mathrm{air}}+\underline{\mathrm{W}}_{\mathrm{I}}\right) \\
& \dot{\underline{v}}_{\mathrm{air}}=\underline{\mathrm{a}}_{\mathrm{B}}+\tilde{\tilde{\omega}} \underline{\underline{v}}_{\mathrm{air}}+\mathrm{H}_{\mathrm{I}}^{\mathrm{B}} \underline{\mathrm{g}}_{I}+ \\
& \tilde{\omega}_{E} \underline{V a i r}-\Omega^{2} \mathrm{RH}_{I}^{\mathrm{B}}\left[\begin{array}{c}
\mathrm{s} \lambda c \lambda \\
0 \\
c^{2} \lambda
\end{array}\right]+\left(2 \tilde{\omega}+\tilde{\omega}_{E}\right) \mathrm{H}_{I}^{B_{I}} \underline{W}_{I}(3-10)
\end{aligned}
$$

The "correction" terms which follow the first lines of equations $(3-14)$ and $(3-10)$, introduce state coupling between them. As will be elaborated, we can reconstruct first the vector $\underline{\mathrm{V}}_{\mathrm{air}}$ and then, under the "flat Earth" assumption, the vector $\underline{x}_{c^{\prime}}$ implying lower-order more robust optimal filtering. This is no longer possible, in particular, as position estimation accuracy requires to add the wind vector $\underline{W}_{I}$ to the state vector. Still, the insight gained by this observation contributed to efficient filter tuning, as shown in Section 3.3. 
To summarize, the angular submodel (from now on referred to as "Model $A$ ") is given by equations $(3-1)$ and $(3-2)$; equations relevant to the translational submodel ("Model B") are $(3-10)$ to $(3-18),(2-3)$ to $(2-8)$ and $(2-12)$ to $(2-18)$. Note that equations such as eq. (2-19) to (2-21) need not be used in the post-flight optimal smoothing and flight path reconstruction because the accelerations $\underline{a}_{B}$ were measured directly.

\subsection{THE OPTIMAL FILTERING AND SMOOTHING ALGORITHMS}

One way to extract measurement noise from experimental data is to pass each record separately through a filter which has been designed to meet an arbitrarily chosen criterion. The Butterworth filter is an example of such a design (Ref. 33). Its analytical structure (low-pass) was hypothesized apriori and the numerical values of its coefficients were obtained via parameter optimization. The chosen performance index, PI for this optimization was minimization of the least square error in following a step input,

$$
P I=\min \text { of }(1 / T) \int_{0}^{\infty} \epsilon^{2} d t
$$

The resulting filter chops off the high-frequency content of the recorded information for each data channel separately 
and without accounting for the particular system's dynamic characteristics.

The Wiener filter is a example of a filter design which incorporates the dynamic model of the physical system (Ref. 34). Its formulation involves solving an integral equation (the Wiener-Hopf equation) through spectral factorization, which gives rise to practical implementation difficulties. These difficulties are alleviated by the Kalman-Bucy filter design (Ref. 35), which converts the problem of extracting experimental data noise into a solution of a differential equation. Its time-domain state-space structure is capable of "one shot" processing of measured time histories of several variables. Also, making use of the "built in" mathematical model of the system, it estimates variables which have not been measured directly. This includes measurement biases thus improving the overall estimation accuracy. For post-flight analysis, even higher accuracy may be achieved by accounting for the "future" information via optimal smoothing (Ref. 36 and 37 ).

As the system mathematical model constitutes a key element in application of these algorithms, it is first restated and then, its stochastic components are discussed. The general state equation, eq. $(2-1)$ is,

$$
\underline{x}=\underline{f}(\underline{x}, \underline{u})+\underline{w}
$$


The measurement equation is,

$$
\underline{\mathrm{z}}=\underline{\mathrm{h}}(\underline{\mathrm{x}}, \underline{\mathrm{u}})+\underline{\mathrm{v}}
$$

The disturbances vector $\underline{w}$ in eq. $(3-20)$ is referred to in the literature as "process noise". The vector $\underline{v}$ in eq. (3-21) is the "measurement noise". Both $\underline{w}$ and $\underline{v}$ were assumed to be "white" and to have a Gaussian (normal) probability density distribution.

As pointed out in section 3.1, the SPIFR mathematical model may be split into lower-order models $A$ and $B$. Proceeding first with model $A$, equation (3-1) may be interpreted as its state model with $\left[\begin{array}{lll}\phi & \theta & \psi\end{array}\right]^{\mathrm{T}}$ constituting the state vector and $\left[\begin{array}{lll}p & q & r\end{array}\right]^{T}$ being the input vector. This formulation prevents noise extraction from the angular rate information, which is used as an input to model B. In order not to create such an inaccuracy effect, the state vector $\underline{x}_{A}^{\prime}$ must be augmented to include $p, q$ and $r$, 
STATE MODEL A

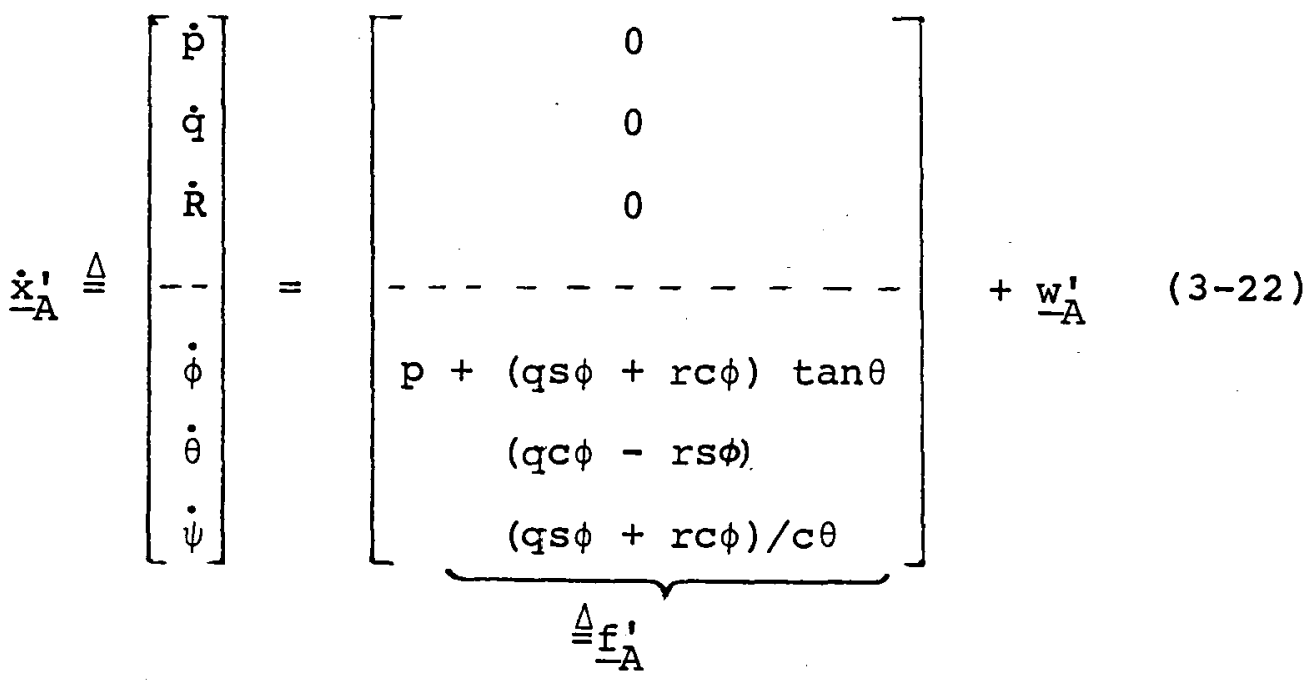

MEASUREMENT MODEL A

$$
\underline{z}_{\mathrm{A}}=\mathrm{H}_{\mathrm{A}}^{\prime} \underline{x}_{\mathrm{A}}^{\prime}+\underline{\mathrm{v}}_{\mathrm{A}}
$$

The process noise vector $\underline{w}_{A}^{\prime}$ reflects random and otherwise unmodeled effects, such as turbulence or engine-induced vibrations,

$$
\underline{w}_{A}^{\prime}=\left[\begin{array}{llllll}
n_{\dot{p}} & n_{\dot{q}} & n_{\dot{r}} n_{\dot{\phi}} n_{\dot{\theta}} & n_{\dot{\psi}}
\end{array}\right]^{T}
$$

The determination of the covariances associated with $\underline{w}_{A}^{\prime}$ is discussed in Section 3.3. The actual measurement noise vector $\underline{v}_{A}$ is,

$$
\underline{v}_{A}=\left[\begin{array}{llllll}
n_{p} & n_{q} & n_{r} & n_{\phi} & n_{\theta} & n_{\psi}
\end{array}\right]^{T}
$$


The measurement vector $\underline{z}_{A}$ in eq. (3-23) contains the measured values of $\underline{x}_{A}^{\prime}$. Thus the measurement model $A$ is linear and the observation matrix $\mathrm{H}_{\mathrm{A}}^{\prime}$ is a $6 \times 6$ identity matrix.

In spite of the desire not to increase the order of the model, bias states (different errors and slowly varying gyro drifts) may need to be estimated. The flight test procedure was designed to minimize this effect. Careful calibration can provide practically error-free scale-factors. Allowing for sufficient warm-up time for the inertial sensors, their outputs were recorded while the airplane was stationary on the runway prior to takeoff and also in between missions, while the aircraft touched down and ran along the runway for about 25 sec, before getting again airborne. Mean values and standard deviations based on these data records were used to reduce the remnant measurement biases. For example, the angular rates have to be zero while stationary on runway, and the $x_{B}-y_{B}$ plane may be accurately levelled to render zero values for pitch and roll angles. Unlike these angles which are measured with respect to the instantaneous LLLN tangent plane (continuous alignment with the local vertical), local North reference and heading drift errors may not be estimated apriori to a sufficient degree of accuracy. Thus, one more state must be added to Model $A$ and eq. (3-22) and (3-23) have to be modified, 


$$
\begin{aligned}
& \underline{\underline{x}}_{\mathrm{A}} \triangleq\left[\begin{array}{c}
= \\
7 \times 1 \\
-- \\
\dot{\underline{x}}_{\mathrm{A}}^{\prime}
\end{array}\right]=\left[\begin{array}{c}
\underline{f}_{\mathrm{A}}^{\prime} \\
-- \\
0
\end{array}\right]+\underbrace{\left[\begin{array}{c}
\mathrm{w}_{-\mathrm{A}}^{\prime} \\
-- \\
\mathrm{n}_{\dot{\mathrm{b}}}
\end{array}\right]}_{\triangleq_{\underline{w}_{\mathrm{A}}}}
\end{aligned}
$$

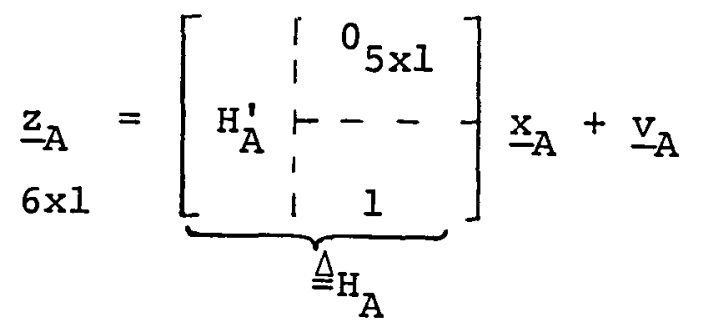

The bias, $b_{\psi}$ was modeled as a random walk process for the estimator. As pointed out in Ref. 38, there is a practical equivalence between the statistics of an exponentially-correlated random process and a random walk process. The random walk model tends to result in slightly pessimistic filter error statistics, which provides a safety margin. The random walk modeling approach is advantageous, because a single state variable can be used to model the sum of the bias plus long-correlation-time errors. This argument was also applied to wind and distance measurement errors in the context of Model B.

Before presenting the equations of the filtering and smoothing algorithms, the appropriate formulation for the translational model, Model $B$, is now derived. It is assumed that having processed the angular information, the time-histories, 


$$
\hat{p}(t), \hat{q}(t), \hat{r}(t) ; \hat{\phi}(t), \hat{\theta}(t), \hat{\psi}(t)
$$

and the associated matrices,

$$
\hat{H_{B}^{I}}(t), \hat{H_{I}^{B}}(t), \hat{\tilde{\omega}}(t)
$$

are given. Based on equations $(3-10)$ to $(3-18),(2-3)$ to $(2-8)$ and $(2-12)$ to $(2-18)$, model $B$ may be recast as follows :

STATE MODEL B

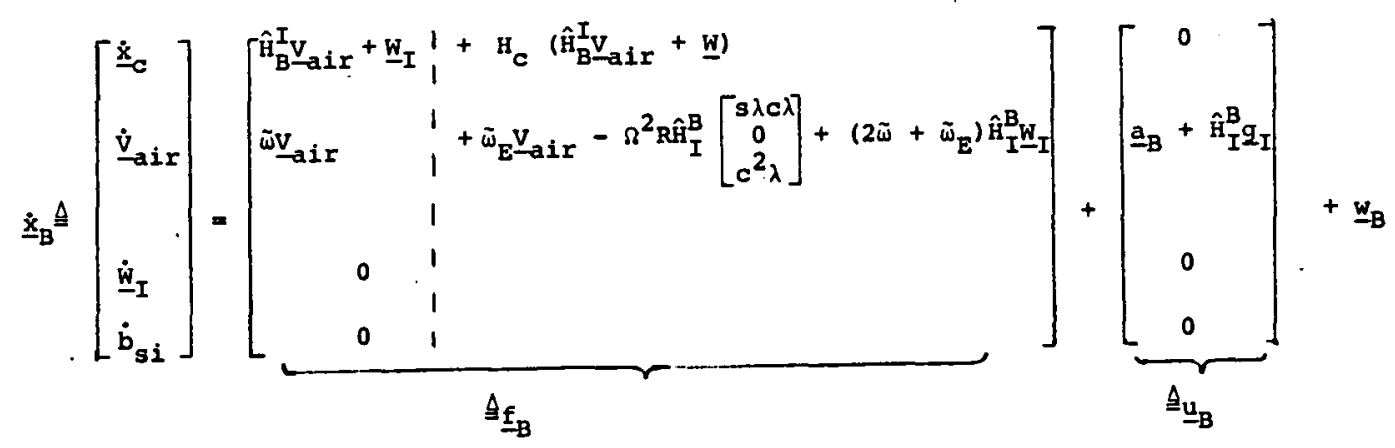

MEASUREMENT MODEL B

$$
\underline{z}_{B} \triangleq\left[\begin{array}{c}
v \\
\alpha \\
\beta \\
h \\
r_{s i}
\end{array}\right]=\left[\begin{array}{l}
\left(u^{2}+v^{2}+w^{2}\right)^{1 / 2} \\
\tan ^{-1}(w / u) \\
\tan ^{-1}(v / u) \\
-z_{I} \\
f\left(\underline{x}_{C} ; \text { eq } \cdot(3-17) \text { and }(3-18)\right)+b_{s i}
\end{array}\right]+\underline{v}_{B}(3-29)
$$


The components of the wind vector $\underline{W}_{I}$ were appended to the state vector. The modeling. of $\mathrm{W}_{\mathrm{I}}$ was as a random walk process (Ref. 38). Following the pre-flight procedure outlined earlier in this section, the biases of the altitude, airspeed and aerodynamic angles were extracted apriori. The $\alpha-$ and $\beta$-vane deflections are defined in body axes and may be calibrated accurately. A previous research effort determined the correlation curves between the geometric vane deflections and the actual aerodynamic angles (Ref. 39). The airspeed sensor was located so as to minimize the local flow effect and the calibration constants corrected for the remnant errors. The biases associated with the range measurements are of a more complicated nature. They depend both on inaccuracies associated with the individual DME stations and with the airborne interrogator. There are also weak timedependent effects associated with the aircraft/station relative geometry (Ref. 40). To account for the above, biastype states $b_{s i}$ must be added to $x_{B}$, and they were modeled as random walk processes (Ref. 38 ).

Vector $\underline{u}_{B}$ is an input to state model $B$. $\underline{a}_{B}$ consists of the acceleration measurements. The associated random measurement error vector $\underline{n}_{a}$ was included in the process noise vector $\underline{w}_{B}$, along with the otherwise unmodeled turbulence effects. Thus, all the components of $\underline{w}_{B}$ may have nonzero values. The determination of the covariances associated with 
$\underline{w}_{B}$ is discussed in Section 3.3. Note that both $\underline{w}_{A}$ and $\underline{w}_{B}$ possess physically significant nonzero values. As pointed out in Ref. 41 and 42 , presence of process-noise-free states in the system's model may cause the Kalman filter algorithm to diverge. The actual measurement noise vector $\mathrm{v}_{B}$ is,

$$
\underline{v}_{B}=\left[\begin{array}{lllll}
n_{V} & n_{a} & n_{\beta} & n_{h} & n_{s i}
\end{array}\right]^{T}
$$

Unlike Model $A$, for which the $\underline{x}_{A}$ components were smoothed optimally, applied to Model B the Kalman filter reconstructed components of $\underline{x}_{B}$ that were not measured directly. Examination of equations $(3-22)$ to $(3-30)$ shows that both models $A$ and $B$ are nonlinear. Thus the Extended Kalman Filter (EKF) algorithm has to be applied (Ref. 37). Then, the optimal smoother algorithm was implemented as a combination of forward- and backward-running Kalman filters. First the filtering algorithm is discussed and then the smoother equations are presented, in a computationally-more-efficient formulation than the literally-forward-backward filter.

The EKF algorithm constitutes an adaptation of the linear Kalman filter theory to nonlinear situations. It propagates the nonlinear dynamic model between measurements and utilizes a locally-linearized model for the measurement updates. The discrete formulation of the EKF, which is a re- 
cursive algebraic algorithm, was implemented in this research. It was applied to the dynamic model of a system, which consists of the state model (eq. (3-20)) and of the measurement model (eq. $(3-21)$ ). The propagation of the estimated states $x$ and of the state covariance matrix $P$ between measurements uses,

$$
\hat{\dot{x}}(t)=\underline{f}[\underline{x}(t), \underline{u}(t)]
$$

and

$$
\mathrm{P}_{\mathrm{k}}(-)=\Phi_{\mathrm{k}-1} \mathrm{P}_{\mathrm{k}-1}(+) \Phi_{\mathrm{k}-1}^{\mathrm{T}}+\mathrm{Q}_{\mathrm{k}-1}
$$

To minimize accumulation of inaccuracies, eq. (3-31) was propagated from $t_{k-1}$ to $t_{k}$ with a fourth-order Runge-Kutta numerical integration scheme (as in Ref. 23) and an integration step of one twentieth of the time interval between measurements, $\Delta t$. The state covariance matrix $P$ is propagated from its value $\mathrm{P}_{\mathrm{k}-1}(+)$, which is a post -measurement (+) update at time step $k-1$, to the pre -measurement (-) value at time step $k$. Matrix $Q_{k-1}$ is the process noise covariance matrix. Assuming the process noise is white, $Q_{k-1}$ is obtained from the diagonal matrix $Q(t)$, which consists of the power spectral densities associated with the process noise vector ( $\underline{w}_{A}$ in eq. $(3-26)$ and $\underline{w}_{B}$ in eq. $\left.(3-28)\right)$. Using trapezoidal integration (Ref. 38), 


$$
Q_{k-1}=0.5 \Delta t\left[\Phi_{k-1} Q_{k-2} \Phi_{k-1}^{T}+Q(t)\right]
$$

Substituting eq. (3-33) into eq. (3-32),

$$
\mathrm{P}_{\mathrm{k}}(-)=\Phi_{\mathrm{k}-1}\left[\mathrm{P}_{\mathrm{k}-1}(+)+0.5 \Delta t \mathrm{Q}_{\mathrm{k}-2}\right] \Phi_{\mathrm{k}-1}^{\mathrm{T}}+0.5 \Delta t Q(t)(3-34)
$$

Matrix $\Phi$ is the transition matrix obtained after local linearization of eq. (3-20) into,

$$
\underline{\dot{x}}=\mathrm{F} \underline{\mathrm{x}}+\mathrm{G} \underline{\underline{u}}+\mathrm{L} \underline{\mathrm{w}}
$$

Although rigorously, $\Phi=e^{F \Delta t}=\Sigma(F \Delta t)^{i} / i l$, Ref. 43 suggests that $i_{\text {upper }}=36$ is sufficient for all practical purposes. This was verified via the generic simulation described in section 3.3. In order not to generate inaccuracies due to numerical differentiation, analytical derivation of the Jacobian matrices F, G, and $L$ was carried out for both models $A$ and $B$; this derivation is documented in Appendix C. Note also that in both models $A$ and $B$ the process noise $\underline{w}$ is additive, making the matrices $G$ and $L$ (in eq. (3-35)) identity matrices. This was used in eq. (3-32).

The Kalman gain matrix $\mathrm{k}_{\mathrm{k}}$ is,

$$
K_{k}=P_{k}(-) H_{k}^{T}\left[H_{k} P_{k}(-) H_{k}^{T}+R_{k}\right]^{-1}
$$


Matrix $R_{k}$ is the measurement noise covariance matrix associated with measurement noise vector $\left(\underline{v}_{A}\right.$ in eq. $(3-27)$ and $\underline{v}_{B}$ in eq. (3-29)). Under the assumption that the measurements are uncorrelated white sequences, matrix $R_{k}$ is diagonal. The observation matrix $\mathrm{H}_{\mathrm{k}}$ is obtained by local Iinearization of eq. $(3-21)$ as,

$$
\underline{\mathrm{z}}_{\mathrm{k}}=\mathrm{H}_{\mathrm{k}} \underline{\mathrm{x}}_{\mathrm{k}}+\underline{\mathrm{v}}_{\mathrm{k}}
$$

As the state equation Jacobians of eq. (3-35), $\mathrm{H}_{\mathrm{k}}$ was derived analytically for both models $A$ and $B$ (Appendix $C$ ).

The state and covariance propagation results are updated accounting for measurements,

$$
\begin{aligned}
& \underline{x}_{k}(+)=\underline{x}+K_{k}\left[\underline{z}_{k}-\underline{h}_{k}(\underline{x})\right] \\
& P_{k}(+)=\left[I-K_{k} H_{k}\right] p_{k}(-)
\end{aligned}
$$

Equations (3-31) to (3-39) represent the classical formulation of the Kalman filter algorithm. However, as pointed out in Ref. 42, this formulation may diverge numerically due to computer roundoff. The effects of numerical errors are generally manifested in the appearance of computed covariance matrices that fail to stay nonnegative-definite. There 
are two distinct approaches to the solution of this problem; one is to improve on the classical filter formulation, and the other is to manipulate the weighted square-roots of the covariance matrices. The improvement of the performance of the classical formulation involves:

* Periodic symmetrization of the off-diagonal terms of the covariance matrix (as in Ref. 38) by averaging;

* Replacement of eq. (3-39) by the more general expression, referred to as the "stabilized Kalman" algorithm (Ref. 44),

$$
\mathrm{P}_{\mathrm{k}}(+)=\left[I-\mathrm{K}_{\mathrm{k}} \mathrm{H}_{\mathrm{k}}\right] \mathrm{P}_{\mathrm{k}}(-)\left[I-\mathrm{K}_{\mathrm{k}} \mathrm{H}_{\mathrm{k}}\right]^{\mathrm{T}}+\mathrm{K}_{\mathrm{k}} \mathrm{R}_{\mathrm{k}} \mathrm{K}_{\mathrm{k}}^{\mathrm{T}}
$$

An excellent comparison study between the two approaches is presented by Thornton and Bierman (Ref. 45). Their results indicate that for single-precision applications (in particular, real-time filtering), the weighted-square-root or the U-D factorization update algorithm is superior in accuracy. However, when double-precision was employed, both approaches gave results that were practically identical. The difference in computer execution time also was insignificant. As the SPIFR post-flight analysis was conducted on an IBM 4341 computer that uses a double-precision FORTRAN compiler only, the symmetrized "stabilized Kalman" algorithm was implemented. 
To summarize, the filter processing of the raw data provides the state and covariance estimates before and after the measurement update, $\underline{x}_{k}(-)$ and $\underline{x}_{k}(+)$, plus. $P_{k}(-)$ and $\mathrm{P}_{\mathrm{k}}(+)$. The smoothing algorithm derived below, requires storage of these filtering results. It uses this information as input; running backwards in time, it produces improved estimates of the states, $\underline{x}_{k} / n^{\prime}$ and of the covariance matrix, $\mathrm{P}_{\mathrm{k} / \mathrm{n}}$

At least three types of smoothing algorithms can be considered. Fixed-point smoothing is concerned with achieving smoothed estimates of a signal $\underline{x}_{k}$ for some fixed point $k$; fixed-lag smoothing introduces a fixed delay between signal reception and the availability of its estimate; fixed-interval smoothing is mainly concerned with off-line smoothing of experimental data. The latter is the most appropriate formulation for accurate post-flight flight path reconstruction. Following the classical work by Rauch, Tung and Striebel (Ref. 46), who developed the sequential algorithms for discrete-time optimal smoothing, several other researchers looked into the fixed-interval smoothing problem (e.g., References 47, 48 and 49). Bach (Ref. 49) based his approach on variational minimization of error; Fraser's (Ref. 47) important contribution is the derivation of the smoothability condition, which reduces computational cost without compromising the smoothing accuracy. The formulation by 
Rauch, Tung, and Striebel was implemented in the present research. It does not require the use of the original data records plus the measurement equation (whose parameters have to be continuously recomputed in the nonlinear case)-also through the smoothing pass. Fraser's smoothability condition also was applied.

The first step is the computation of the state matrix $\mathrm{F}_{\mathrm{k}^{\prime}}$

$$
F_{k}=f\left[\underline{x}_{k}(+)\right]
$$

The state matrix $F_{k}$ is used to calculate the state transition matrix $\Phi_{k}$ (see comment following eq. $(3-35)$ ). The state transition matrix and the input covariance matrices render matrix $A_{k^{\prime}}$

$$
A_{k}=P_{k}(+) \Phi_{k}^{T} P_{k+1}^{-1}(-)
$$

Using the input state estimates $\hat{x}_{k}(-)$ and $\hat{x}_{k}(+)$ and the associated covariance matrices $\mathrm{P}_{\mathrm{k}}(-)$ and $\mathrm{P}_{\mathrm{k}}(+)$ along with $A_{k}$, the smoothed and reconstructed states $\underline{x}_{k / n}$ are obtained,

$$
\hat{x}_{\mathrm{k} / \mathrm{n}}=\hat{\hat{x}}_{\mathrm{k}}(+)+\hat{A}_{\mathrm{k}}\left[\hat{\underline{x}}_{\mathrm{k}+1 / \mathrm{n}}-\hat{\hat{x}}_{\mathrm{k}+1}(-)\right]
$$

with 


$$
\underline{x}_{n / n}=\hat{x}_{n}(t)
$$

The accompanying covariance matrix is given by,

$$
P_{k / n}=P_{k}(+)+A_{k}\left[P_{k+1 / n}-P_{k+1}(-)\right] A_{k}^{T}
$$

with

$$
P_{n / n}=P_{n}(t)
$$

This algorithm, which performs post-flight data smoothing and flight path reconstruction, was coded in FORTRAN (Ref. 17). The optimal filter was tuned using a trajectory simulation, and the efficiency of the smoother was improved, applying the smoothability condition (Ref. 47). These activities along with implementation of the optimal estimation algorithm to actual SPIFR flight-test data, are reported in the next section. 
3.3 FILTER TUNING AND IMPLEMENTATION OF THE OPTIMAL FLIGHT PATH RECONSTRUCTION ALGORITHM

The required inputs for the optimal flight path reconstruction algorithm are the measured time series and the driving noise statistics $Q$ and $R$. In most cases, estimates of the measurement noise-covariances, $R_{k}$ can be derived from the sensor manufacturers' specifications. The more difficult problem is the determination of the elements of the matrix $Q(t)$; because they have to reflect the system's modeling uncertainties along with the actual process noise; this problem is referred to as filter tuning.

As pointed out in Ref. 50, the steady-state dynamic response properties of the Kalman filter may be deduced from the eigenvalues of the estimation error differential equation; an example of this is presented by Bryson and Widnall for a baro-inertial altitude estimator (Ref. 51 and 52 ). They show that these eigenvalues of the steady-state optimal estimator are functions of the ratios of elements in the spectral density matrix of the state-driving noise, $Q(t)$ and in the spectral density matrix of the measurement noise, $R(t)$. Thus the $Q$ matrix elements may be perceived as filter design parameters, and the $R$ matrix is given apriori, as mentioned at the beginning of this section. 
Before discussing the filter tuning methodology and the criteria for an appropriate choise of the elements of $Q$, it should be kept in mind that the above argument rigorously applies only to the steady-state optimal estimator case. In this case, where system and measurement dynamics are linear and time-invariant and the driving noise statistics are stationary ( $Q, R$ are not functions of time), the filtering process may reach a "steady state" wherein the state covariance matrix $\mathrm{P}$ is constant. For this to happen, the complete observability and controllability conditions must be fulfilled (Ref. 37). Then, introducing $\dot{P}=0$ into the continuoustime Riccati formulation, important physical insight may be gained,

$$
\mathrm{FP}+\mathrm{PF}^{\mathrm{T}}+\mathrm{Q}-\mathrm{PH}^{\mathrm{T}} \mathrm{R}^{-1} \mathrm{HP}=0
$$

In this steady state, the rate in which uncertainty builds up, Q, is just balanced by the rate at which new information enters the system, $\mathrm{PH}^{\mathrm{T}} \mathrm{R}^{-1} \mathrm{HP}$ and the system dissipation due to damping (expressed in F). Although the SPIFR dynamic model is nonlinear and time-varying, the numerical values of the elements of the linearization Jacobians are bounded and vary slowly with time. Thus, appropriate tuning may render a practically steady-state filter performance. 
The filter tuning process consisted of two distinct steps: (a)initial choise of numerical values for the Q-elements, and (b)iterative procedure of convergence to their final values, which satisfy certain performance criteria. This iterative procedure involved repeated implementation of the EKF algorithm to simulated data records, which were created by a flight path generation computer program (Appendix D). The input options to this generic flight path simulator included the effects of low-frequency wind gusts and of biases, plus random measurement errors and disturbances. The pseudo-random-number generator, which creates the Gaussian probability distribution (Ref. 53), produced a point every 50 milliseconds for each variable. As in flight testing, actually used were the points corresponding to the A/D sampling instances (Appendix A). This computer code rendered both the nominal noise-free trajectory and the corrupted data records. The acceptability of a set of values for the elements of the matrix $Q$ was judged by comparing these simulated data records to the time histories obtained by filtering and then smoothing the corrupted data. Quantitatively, measurement residuals were compared with the estimated states' covariances, as will be shown later in this section.

The filter was tuned with a $60-\mathrm{sec}$ straight flight path segment (Fig. 3.2); then its performance was verified with a coordinated climbing turn maneuver (Fig. 3.3). The lat- 
ter may be interpreted as a holding pattern maneuver, which is characteristic of SPIFR flight and which contains larger parameter variations. The asteriks (*) in Figures 3.2 and 3.3 denote the locations of the navigational ground stations, which provide range and azimuth information. The numerical values in both are the inputs to the generic simulation. They were obtained via a steady-state (equilibrium flight conditions) analysis. Both figures are schematic horizontal projections of the flight trajectories, referring to the nominal (bias- and noise- free) cases.

The following measurement noise standard deviation values characterise the sensors employed in the research. They are based on manufacturers' data and were verified after integration into the experimental setup, as described in section 3.2.

$$
\begin{aligned}
& \sigma_{p}=\sigma_{q}=\sigma_{r}=0.15 \mathrm{deg} / \mathrm{sec} \\
& \sigma_{\phi}=\sigma_{\theta}=\sigma_{\Psi}=0.15 \mathrm{deg}
\end{aligned}
$$




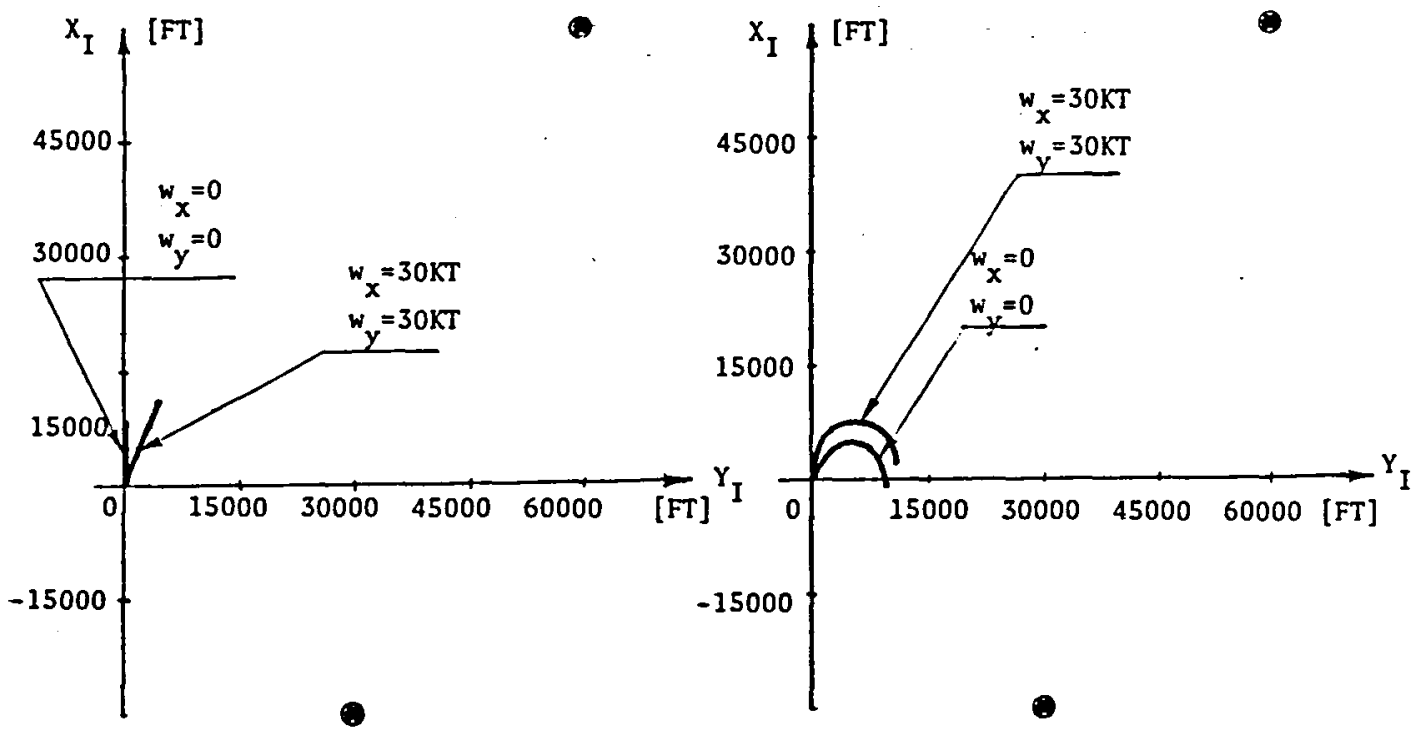

$h_{0}=500 \mathrm{FT}, v_{0}=325.6 \mathrm{FPS}, \gamma_{0}=0, \theta_{0}=5.5^{\circ}$,

$h_{0}=116 \mathrm{FT}, v_{0}=125.6 \mathrm{FPS}, \gamma_{0}=4.5^{\circ}, \theta_{0}=10^{\circ}$,

$\phi=\psi=p=q=I=a_{y}=0, a_{x}=0.096 " g^{\prime \prime}, a_{2}=-0.994 " \mathrm{~g} "$

$\phi_{0}=12.7^{\circ}, \psi_{0}=a_{y}=0, p=-0.5^{\circ} / \mathrm{SEC}, q=0.6^{\circ} / \mathrm{SEC}$,

$I=2.86^{\circ} / \mathrm{SEC}, a_{x}=0.179^{\prime \prime} \mathrm{g}^{\prime \prime}, a_{z}=1.003^{\prime \prime} \mathrm{g}^{\prime \prime}$

Figure 3.2: Longitudinal Flight Path Segment

Figure 3.3: Coordinated Climbing Turn

$$
\begin{aligned}
& \sigma_{\mathrm{V}}=1.0 \mathrm{fps} \\
& \sigma_{a}=\sigma_{\beta}=0.30 \mathrm{deg} \\
& \sigma_{\mathrm{h}}=4.0 \mathrm{ft} \\
& \sigma_{\mathrm{DME}}=600 \mathrm{ft} \\
& \sigma_{\mathrm{ax}}=\sigma_{\mathrm{ay}}=\sigma_{\mathrm{az}}=0.02 \mathrm{~g} "
\end{aligned}
$$

These values were used with the random-number generator in the generic simulation and with the measurement covariance 
matrix in the optimal filtering algorithm. As pointed out in Section 3.2, $\sigma_{a x} \sigma_{a y}$ and $\sigma_{a z}$ were interpreted as part of the process noise covariance matrix.

The value of $\sigma_{D M E}$ in eq. $(3-49)$ is based on eq. $(2-98)$ in conjunction with the anticipated range of DME distance measurement values from points on SPIFR trajectories (Fig. 2.4 to 2.7$)$ to the ground stations engaged in the flight tests (the detailed list of the stations' coordinates is given in Appendix D). The DME stations' bias was assumed to be $500 \mathrm{ft}$ (Ref. 31), and the value of $1.5 \mathrm{deg}$ was taken for the directional gyro error, $b_{\psi}$.

Starting with the actual tuning for model $A$, the initial choice of the numerical values of the Q-elements has to be made. One may observe from eq. (3-22) that the state vector $\underline{x}_{A}$ consists of body angular rates and of Euler angles. With regard to variations in the Euler angle states, higher time constants are involved and the low-frequency range is of interest. Thus $\sigma_{\phi}^{2}, \sigma_{\psi}^{2}$ and $\sigma_{\theta}^{2}$ of eq. (3-48) must be higher than the respective $Q(t)$-elements. On the other hand, the latter should not be too low, as this would mean that the angle measurements are of relatively poor quality and should be ignored, instead of being used in an optimal fashion. As an initial choice, values of measurement covariances, $\sigma_{\mathrm{p}}^{2} \sigma_{\mathrm{q}}^{2}$ and $\sigma_{\mathrm{r}}^{2}$ were taken. 
In contrast with the Euler angle states, a higher-frequency range is of interest with regard to the angular rate states. Covariances $\sigma_{p^{\prime}}^{2} \sigma_{q^{\prime}}^{2}$ and $\sigma_{r}^{2}$ may not be higher than the respective $Q(t)$-elements. This also is due to the frequency $\omega^{2}$-weighting in power spectral densities of these Q-elements (Ref. 54). On the other hand, they should not be too high, as this may prevent filtering out the high-frequency measurement noise. The SPIFR research, in particular, focused on the low-frequency dynamic response and even the "short-period" frequency range (about 2 cps : Ref. 21) is irrelevant. This frequency-domain line of reasoning renders trends of variation for the filter tuning iterations.

The results of the tuning of the optimal filter for Model A are summarized in Table 3.1. This table represents the translation of the theoretical $Q-v e r s u s-R$ tuning reasoning into a practical implementation procedure. Given the $R_{k} n u-$ merical values (column 2), values for $Q$ (column 6) were chosen and the optimal filtering and smoothing algorithms were applied. Along with the filtered and smoothed state variables' time histories, the respective covariance matrices $P_{f}$ and $P_{S}$ are tabulated (columns 4 and 5 ). Comparison of their diagonal terms shows the improvement over filtering gained by smoothing. To complete the analysis of the effects of the particular Q-elements tuning choice, the sta- 
tistics of actual measurement residuals was used to obtain a quantitative filter performance criterion. The vector of measurement residuals, $\Delta z$ is given by,

$$
\Delta z \quad=\underline{z}-h(\underline{x})
$$

The mean square values of $\Delta \mathrm{z}, \widehat{\sigma}^{2} \mathrm{zi}$ (column 3 in Table 3.1 ) are,

$$
\hat{\sigma}_{z i}^{2}=\tilde{\Sigma}\left(\Delta z \Delta z^{T}\right)_{i i} /(N-1)
$$

Following the argument at the beginning of this section, we want to avoid both,

$$
\hat{\sigma}^{2} \ll R_{k i i} \quad ; \quad \hat{P}_{i i}=R_{k i i}
$$

and

$$
\hat{\sigma}_{z i}=R_{k i i} \quad ; \quad \hat{P}_{i i} \ll R_{k i i}
$$

should be avoided. In the first case the algorithm performs measurement-following and in the second, it imposes heavy filtering, minimizing the measurement input. The objective is to filter out the noise without "filtering out" the actual information along with it. Sets of results as the one of Table 3.1 were complemented with visual inspection of nominal-versus-corrupted-versus-filtered-versus-smoothed time histories (examples are given at the end of this section) to decide on the final numerical values for the Q-elements. 
TABLE 3.1

Filter Tuning Results for Model A

\begin{tabular}{|c|c|c|c|c|c|}
\hline $\begin{array}{l}\text { STATE } \\
\text { VARIABLES }\end{array}$ & $\begin{array}{c}\text { MEASUREMENT } \\
\text { COVARANCES } \\
\text { (DIAGONALS OF } \mathrm{R}_{\mathrm{k}} \text { ) }\end{array}$ & $\begin{array}{l}\text { MEASUREMUENT } \\
\text { RES DUUAL } \\
\text { COVARIANCES } \\
\partial_{\Delta Z 1}^{2}\end{array}$ & $\begin{array}{l}\text { FILTERED STATE } \\
\text { COVARINANES } \\
\text { (DIAGONALS OF } \hat{\mathrm{P}}_{\mathrm{f}} \text { ) }\end{array}$ & $\begin{array}{l}\text { SMOOTHED STATE } \\
\text { COVARIANCES } \\
\text { (DIAGORALS OF } P_{\mathbf{a}} \text { ) }\end{array}$ & $\begin{array}{l}\text { PROCESS NOISE } \\
\text { POWER SPECTRAI } \\
\text { DENSITIES } \\
\text { (DIAGONALS OF Q) }\end{array}$ \\
\hline $\mathbf{p}$ & $7.0 \times 10^{-6}$ & $3.8 \times 10^{-6}$ & $4.5 \times 10^{-6}$ & $3.8 \times 10^{-6}$ & $9.0 \times 10^{-6}$ \\
\hline$q$ & $7.0 \times 10^{-6}$ & $2.0 \times 10^{-6}$ & $4.5 \times 10^{-6}$ & $3.8 \times 10^{-6}$ & $4.0 \times 10^{-6}$ \\
\hline $\mathbf{r}$ & $7.0 \times 10^{-6}$ & $3.8 \times 10^{-6}$ & $4.5 \times 10^{-6}$ & $3.8 \times 10^{-6}$ & $9.0 \times 10^{-6}$ \\
\hline$-\cdots-$ & --- & --- & - & $-\quad-$ & --- \\
\hline$\phi$ & $7.0 \times 10^{-6}$ & $4.5 \times 10^{-6}$ & $4.5 \times 10^{-6}$ & $3.8 \times 10^{-6}$ & $0.5 \times 10^{-6}$ \\
\hline$\theta$ & $7.0 \times 10^{-6}$ & $3.1 \times 10^{-6}$ & $4.5 \times 10^{-6}$ & $3.8 \times 10^{-6}$ & $0.5 \times 10^{-6}$ \\
\hline$\psi$ & $7.0 \times 10^{-6}$ & $5.2 \times 10^{-6}$ & $4.5 \times 10^{-6}$ & $3.8 \times 10^{-6}$ & $0.5 \times 10^{-6}$ \\
\hline
\end{tabular}

The last state variable, with respect to which Model A remains to be tuned, is $b_{\psi}$ (eq. $\left.(3-26)\right)$. As there was no direct measurement associated with the estimation of $b_{\psi}$, the iterative procedure was employed. As a result,

$$
Q(t)_{b_{\psi}}=Q_{77}=2.5 \times 10^{-7}
$$

The penalty for the need to estimate $b_{\psi}$ was some reduction in the accuracy of the estimation of $\psi$ itself. However, as 
obvious from eq. (3-22), this did not affect practically the estimation of the other state variables of model $A$, as the yaw angle is an "open-loop" navigational variable.

Finally, the smoother algorithm of equations (3-41) to (3-46) was applied to the original $6 \times 6$ bias-free mathematical model of eq.' $(3-22)$ and (3-23). Although strictly speaking, the smoothability condition (Ref. 47) applies only to process-noise-free constants, it was verified that one may increase robustness by order reduction with practically no accuracy penalty.

To proceed with the dynamic tuning for model $B$, the "correction" terms in eq. (3-28), which constitute a weak coupling between the flight dynamics and the navigational submodel, are omitted. Thus, equations (3-28) and (3-29) may be partitioned as follows,

$$
\underline{\dot{x}}_{B 1} \triangleq\left[\begin{array}{c}
u \\
\dot{v} \\
\dot{w}
\end{array}\right]=\hat{\tilde{\omega}}\left[\begin{array}{l}
u \\
v \\
x
\end{array}\right]+\left[\begin{array}{l}
a_{x}-s \hat{\theta} g \\
a_{y}+c \hat{\theta} s \hat{\phi} g \\
a_{z}+c \hat{\theta} c \hat{\phi} g
\end{array}\right]+\underline{w}_{B 1}
$$

SUBMODEL

BI

$$
\underline{z}_{B I} \triangleq\left[\begin{array}{l}
v \\
\alpha \\
\beta
\end{array}\right]=\left[\begin{array}{l}
\left(u^{2}+v^{2}+w^{2}\right)^{1 / 2} \\
\tan ^{-1}(w / u) \\
\tan ^{-1}(v / u)
\end{array}\right]+\underline{v}_{B 1}
$$


and,

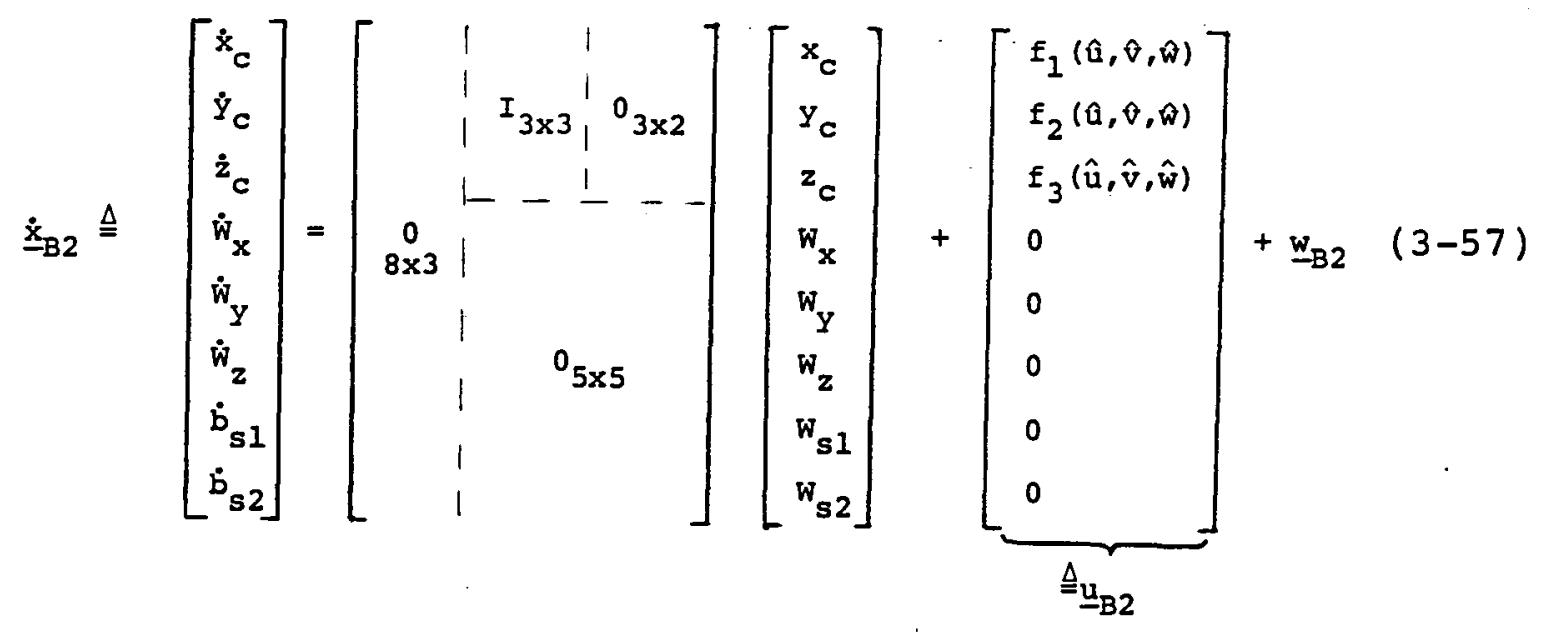

SUBMODEL

B 2

$$
\underline{z}_{B 2} \triangleq\left[\begin{array}{l}
h \\
r_{s 1} \\
r_{s 2}
\end{array}\right]=\left[\begin{array}{l}
-z_{c} \\
f\left(\underline{x}_{c}, \underline{x}_{s 1}+b_{s 1}\right. \\
f\left(\underline{x}_{c}, x_{s 2}\right)+b_{s 2}
\end{array}\right]+\underline{v}_{B 2}
$$

Submodel $\mathrm{Bl}$ is self-contained. To gain more insight, one may equivalence, in an approximate sense, the measurement equations to direct measurements of states (as for Model A). Assuming the angles of attack and of sideslip to be small angles, which holds for the SPIFR flight regime,

$$
\left.\begin{array}{l}
\mathrm{v} \cong \mathrm{u} \\
\alpha \mathrm{u}=\mathrm{w} \\
\dot{\beta \mathrm{u}}=\mathrm{v}
\end{array}\right\}
$$


Variations in $\mathrm{u}$ would affect the $\alpha$ and $\beta$ measurements less than variations in $w$ and $v$, respectively. For an airspeed of 125 fps, e.g., and values of $\sigma_{a}$ and $\sigma_{\beta}$ from eq. (3-49), the equivalent $\sigma_{\mathrm{w}}$ and $\sigma_{\mathrm{v}}$ are,

$$
\sigma_{\mathrm{w}}=\sigma_{\mathrm{v}}=125 \times 0.30 \times 0.01745=0.65 \mathrm{fps}
$$

and,

$$
\begin{aligned}
& \sigma_{\mathrm{u}}=\sigma_{\mathrm{v}}=1.0 \mathrm{fps} \\
& \sigma_{\mathrm{ax}}=\sigma_{\mathrm{ay}}=\sigma_{\mathrm{az}}=0.02 " \mathrm{~g}^{\prime \prime}=0.64 \mathrm{ft} / \mathrm{sec}^{2}
\end{aligned}
$$

Variations in $u$ correspond to a lower frequency range than variations in $w$ or in $v$. This gives a lead for the frequency-domain line of reasoning. Recall that the measurement noise associated with the accelerometers' outputs is interpreted as a component in the process noise vector $\underline{w}_{B I}$. Using the values in eq. $(3-62)$ as a starting point, it may be expected that numerical values of $\sigma_{a y}$ and $\sigma_{a z}$ would need to be increased more (with respect to $\sigma_{w}$ and $\sigma_{v}$ ) than that of $\sigma_{\text {ax }}$ (with respect to $\sigma_{u}$ ). To complete the tuning for submodel Bl, the iterative procedure must now be employed. Before citing the results, a practical implementation issue, which may affect the final tuning results, is now referred to. 
As described in Appendix A, an averaging scheme for onboard compaction of fast variables (angular rates, linear accelerations and aerodynamic angles) was chosen. It is well known (e.g., Ref. 37) that such an approach may result in performance degradation of the Kalman filter due to introduction of nonwhiteness and correlation into the measurement noise sequence. However, the time interval in the averaged sequences was about an order of magnitude lower than the time constant of interest. Thus, the whiteness approximation is justified. With regard to the time correlation introduced into the measurements, its effect is mainly to increase the noise level relative to the reduced measurement noise, $\sigma_{\underline{v}}^{2} / \mathrm{n}$ ( $\mathrm{n}$ is the number of averaged measurements). This additional noise depends on the statistical properties of the state variables associated with those measurements. The solution implemented by Schmidt etal(Ref.55) was to introduce first-order shaping filters with white noise inputs. As already pointed out, an alternative solution may be to tune for an equivalent noise level without changing the model structure. As a guideline, the equivalent noise level may be expected to be within the following bounds:

$$
\sigma_{\underline{v}}^{2} /(n-1) \leq \sigma_{\text {vequivalent }}^{2} \leq \sigma_{\underline{v}}^{2}
$$

Applying the powerful tuning methodology, which regulates the $Q / R$ ratio, the filter performance degradation may be 
minimized. This was verified via numerous Monte-Carlo-type runs (Ref. 56), although this was not an extensive MonteCarlo analysis. Final tuning of submodel Bl rendered,

$$
\begin{aligned}
& \sigma_{w}=\sigma_{v} \cong 0.49 \mathrm{fps} \text { or } \sigma_{a}=\sigma_{\beta}=0.23 \mathrm{deg} \\
& Q(t)_{i i}=0.81
\end{aligned}
$$

With regard to model $A$, the averaging scheme problem was solved by small readjustments of the Q-matrix elements. The numerical values in Table 3.1 account for this effect.

The state vector $\left[\begin{array}{lll}u & v & w\end{array}\right]^{T}$, which was reconstructed via submodel $\mathrm{BI}$, is used now to prepare the deterministic forcing vector $\underline{a}(t)$ - for the tuning of submodel B2 (eq. (3-57) and (3-58)). One may observe from eq. (3-57) that assuming concurrent distance measurements (for tuning purposes) and under zero-wind conditions, the submodel B2 simplifies to static estimation of $\left[\begin{array}{lllllll}x_{c} & y_{C} & z_{c} & b_{s 1} & b_{s 2}\end{array}\right]^{T}$ as $F_{B 2}=0 \quad\left(\Phi_{B 2}=I\right)$. Thus, wind modeling is responsible for the filter dynamics. Applying the $\mathscr{L}^{-1}\left([s I-F]^{-1}\right)$ algorithm and using matrix partitioning and block diagonalization (Ref. 22), the state transition matrix is obtained ( $\Delta t=1 . s e c)$,

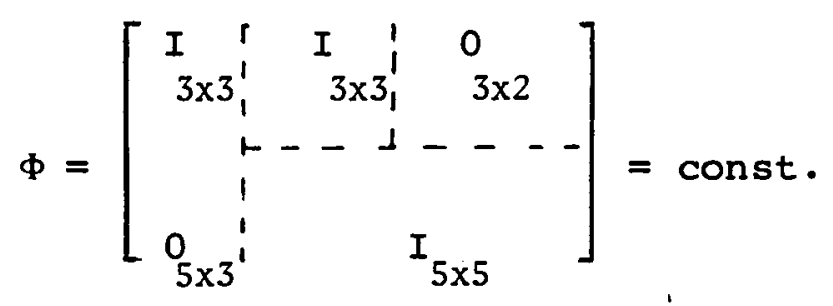


The only state which is directly measured in this submodel is $z_{c}$; thus its tuning is practically uncoupled from the other two states. The latter, $x_{C}$ and $y_{C^{\prime}}$ are related to the range measurements and consistently with eq. (3-49) the fine-tuning renders,

$$
\begin{aligned}
& Q(t)_{x_{c} x_{c}}=Q(t){ }_{y_{c} y_{c}}=100^{2} \\
& Q(t)_{z_{c} z_{c}}=6^{2}
\end{aligned}
$$

The tuning results for model $B$ up to this point are summarized in Table 3.2 .

TABLE 3.2

\begin{tabular}{|c|c|c|c|c|c|}
\hline $\begin{array}{c}\text { STATE } \\
\text { VARIABLES }\end{array}$ & $\begin{array}{c}\text { MEASUREMENT } \\
\text { COVARIANCES } \\
\text { (DIAGONALS OF 'Rk' }\end{array}$ & $\begin{array}{l}\text { MEASUREMENT } \\
\text { RESIDUAL } \\
\text { COVARIANCES } \\
\hat{\sigma}_{\Delta \mathbf{2 1}}^{2}\end{array}$ & $\begin{array}{l}\text { FILTERED STATE } \\
\text { COVARIANCES } \\
\text { (DIAGONALS OF } \hat{\beta}_{f} \text { ) }\end{array}$ & $\begin{array}{l}\text { SMOOTHED STATE } \\
\text { COVARIANCES } \\
\text { (DIAGONALS OF } \hat{P}_{s} \text { ) }\end{array}$ & $\begin{array}{l}\text { PROCESS NOISE } \\
\text { POWER SPECTRAL } \\
\text { DENSITIES } \\
\text { (DIAGONALS OF Q) }\end{array}$ \\
\hline $\mathrm{V}$ & 1.00 & 0.36 & 0.16 & 0.09 & 0.81 \\
\hline$\alpha$ & 0.000016 & 0.000012 & 0.000009 & 0.000008 & 0.81 \\
\hline$B$ & 0.000016 & 0.000012 & 0.000009 & 0.000008 & 0.81 \\
\hline $\mathbf{h}$ & 16.0 & 8.0 & 6.3 & 5.3 & 36.0 \\
\hline$r_{s 1, s 2}^{*}$ & 360000 & 300000 & 62500 & 57600 & 10000 \\
\hline
\end{tabular}

Filter Tuning Results for Model B 
With respect to the remaining wind and DME error states, which were modeled as random walk processes, the tuning was done via the iterative procedure. As mentioned earlier in the section, the range biases may be about $500 \mathrm{ft}$ and the winds-up to 40 knots. The values of the $Q$ elements corresponding to the distance measurement errors were determined to be $10^{2}$. With regard to the wind estimation, the $\mathrm{w}_{z}$ component was assumed to be negligible, within the low-frequency range of interest. The coordinate $z_{c}$ was measured directly and, as verified using the generic simulation, the effect of $\mathrm{w}_{\mathrm{z}}$ on position accuracy is insignificant. Unlike the $\left(z_{c}, w_{z}\right)$-pair, $x_{c}$ and $y_{c}$ may be expected to be sensitive to $W_{x}$ and $W_{y}$ accuracies, in particular as the wind amplitude range is large. A way to cope with the problem is to use the iterative process to obtain the functional relationship between the wind gust level and the respective Q-elements (Fig. 3.4). Then, using this relationship and the meteorological data, namely wind magnitudes $W_{x c}$ and $W_{y c}$ at flight-test altitudes, the $Q$ elements for a specific postflight trajectory reconstruction may be determined.

The position uncertainty may be represented by an ellipsoid, whose half-axes are the square roots of the diagonal elements of the state covariance matrix $\mathrm{P}_{k} \cdot$ This ellipsoid is flattened in the $z_{c}$ direction, as the $z_{c}$ state is meas- 


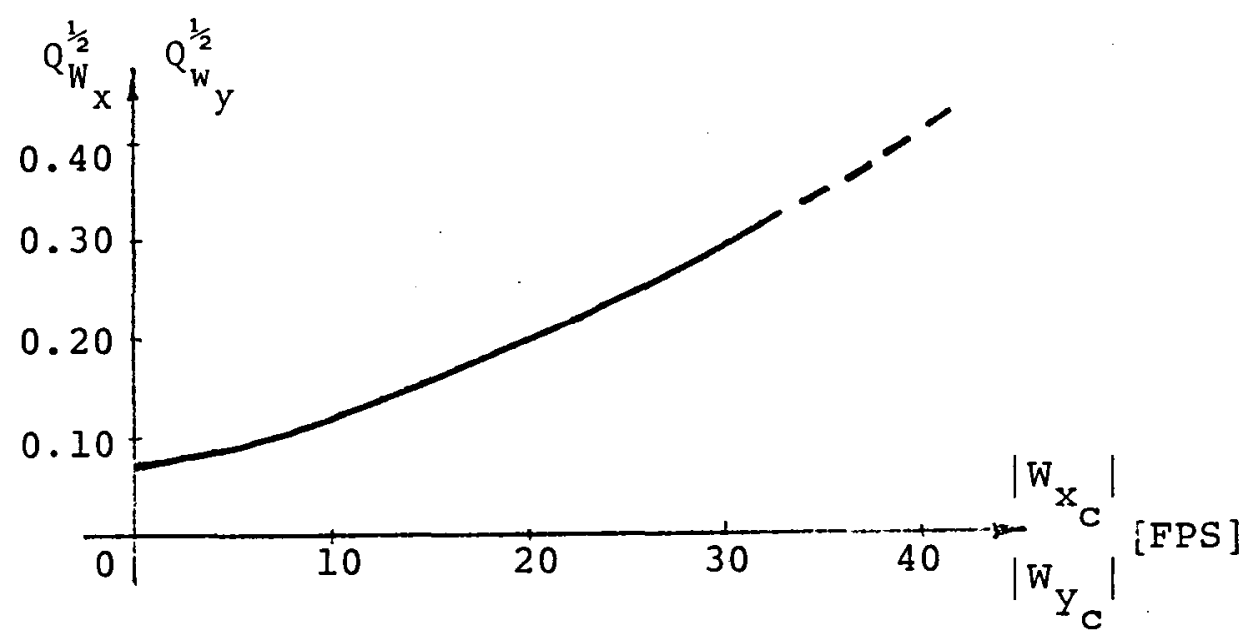

Figure 3.4: Filter Tuning for Wind Estimation

ured directly and with a high degree of accuracy. As mentioned following eq. (3-49), $\sigma_{x c}$ and $\sigma_{y c}$ depend on the geometry of the ground stations with respect to the aircraft (e.g., on distance and on the GDOP effect) and may not be equal to each other. Moreover, the filter will render different $\sigma_{\mathrm{xC}}$ and ${ }_{\mathrm{yc}}$ as a function of the wind intensity level, when it is tuned for a range of distances (Fig. 3.4). In a different context, it has been shown that a wind of 40 knots may degrade the accuracy of position estimation by about 108 (Ref. 57). Thus, for best results, the postflight flight path reconstruction procedure should be interactive and should account for gross variations in distances and in wind intensity. 
The tuning procedure involved several practical implementation issues, such as the switching to a new pair of DME stations and the rare interference of the Morse code sent out by the tuned-in ground stations every thirtieth of a second. Both occurences caused an abrupt saturation of the DME data channel. This is an outlier (or "wild point") problem, i.e., data not due to the physical system's response. As pointed out in Ref. 58, automatic wild point editing schemes may distort the good data. However, due to the high gradients associated with the abrupt channel saturation, the problem could be readily detected, and the last good measurement was extrapolated to obtain estimates of the flight path variables. When the switching to a new pair of stations was completed, a hexidecimal code which identified the new station combination was entered into a specially dedicated data channel (Appendix D). Based on this information, time vectors of station engagements were prepared for input to the filtering program. When the computation switched to a new pair of navigational stations, the off-diagonal elements of the covariance matrix $P_{k}$ were reset to zero (Ref. 31). An example of a case where a large discontinuity in a data channel is physically significant is the change of the yaw angle at the $0 / 360$ deg point; the value of the state variable was shifted accordingly with no covariance resetting. 
Examples of the optimal flight path reconstruction algorithm's application to the generic flight-test data records are given in Fig. 3.5 for the coordinated climbing turn of Fig. 3.3. Plots (a) to (f) of the figure present reconstructed measurements, demonstrating both state variable reconstruction and improvement with respect to data corrupted by noise. The symbol convention used in these plots is: $(+)$ for nominal, ( $(\square)$ for corrupted, $(\nabla)$ for filtered and $(\Delta)$ for smoothed time histories. Line segments are used to link results but they do not imply a functional relationship. ${ }^{1}$

Plots (a) and (b) represent the optimal smoothing of the angular states. As may have been expected, the "derivative" states (e.g.. (b)) are noisier than the "integral" states (e.g., (a)). This distinction may also be applied to the airspeed versus aerodynamic angle measurements, which reflect the atmospheric turbulence effect. As follows from the translational submodel formulation, to reconstruct these measurements (e.g., plots (c) and (d)), the states $u, v$ and w were first estimated. The typical lag introduced by filtering is more apparent in some of the figures; it is then reduced by the smoother. The trajectory reconstruction is represented in plots $(e),(f)$ and $(g)$. Note that optimal smoothing improves the filtered state estimates and also shrinks the position uncertainty ellipsoid.

1 In this program run both range measurements were assumed to be obtained (and corrupted) every second. 

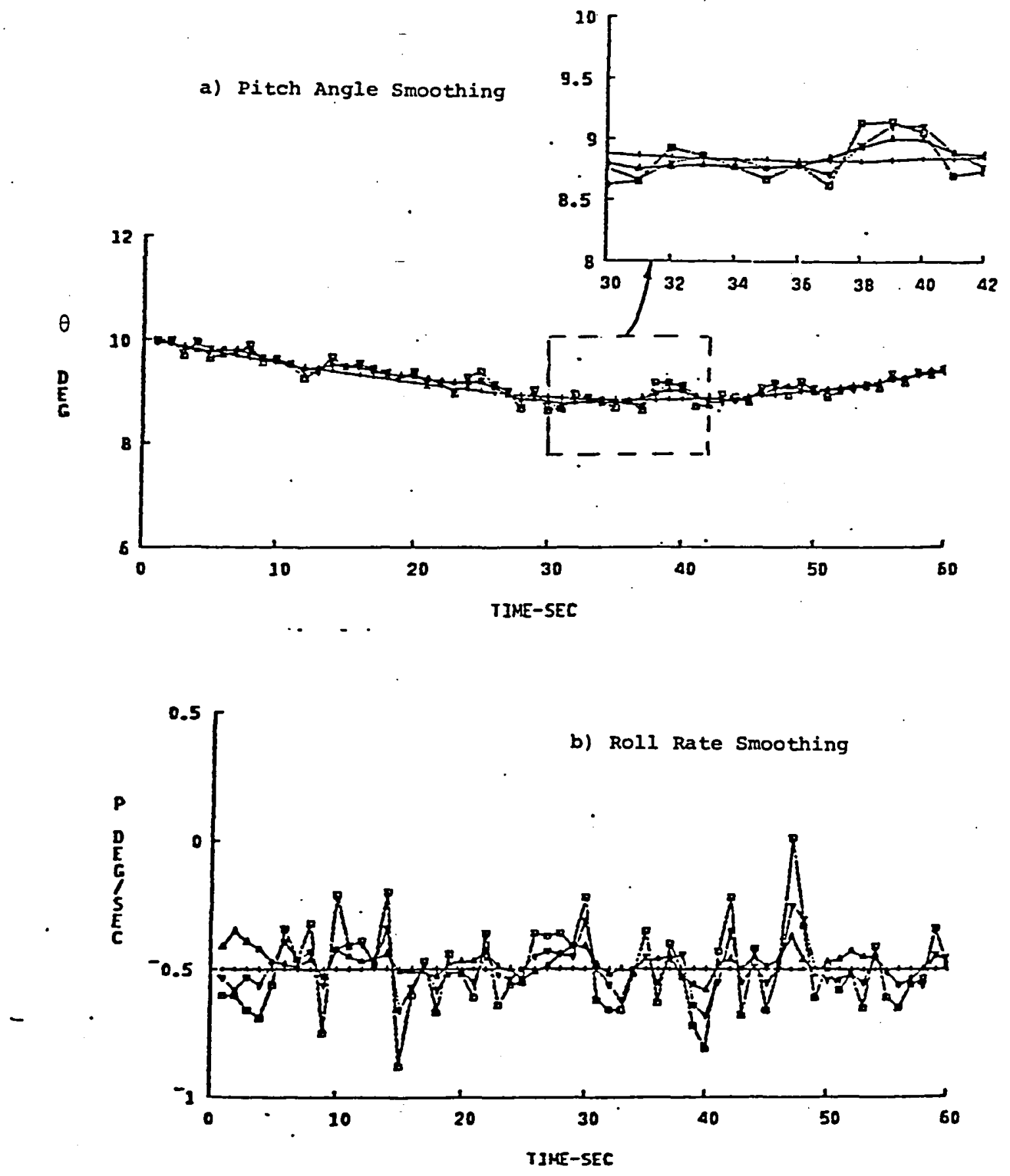

Figure 3.5: Application of the Optimal Flight Path Reconstruction Algorithm to the Climbing Turn Generic Data 

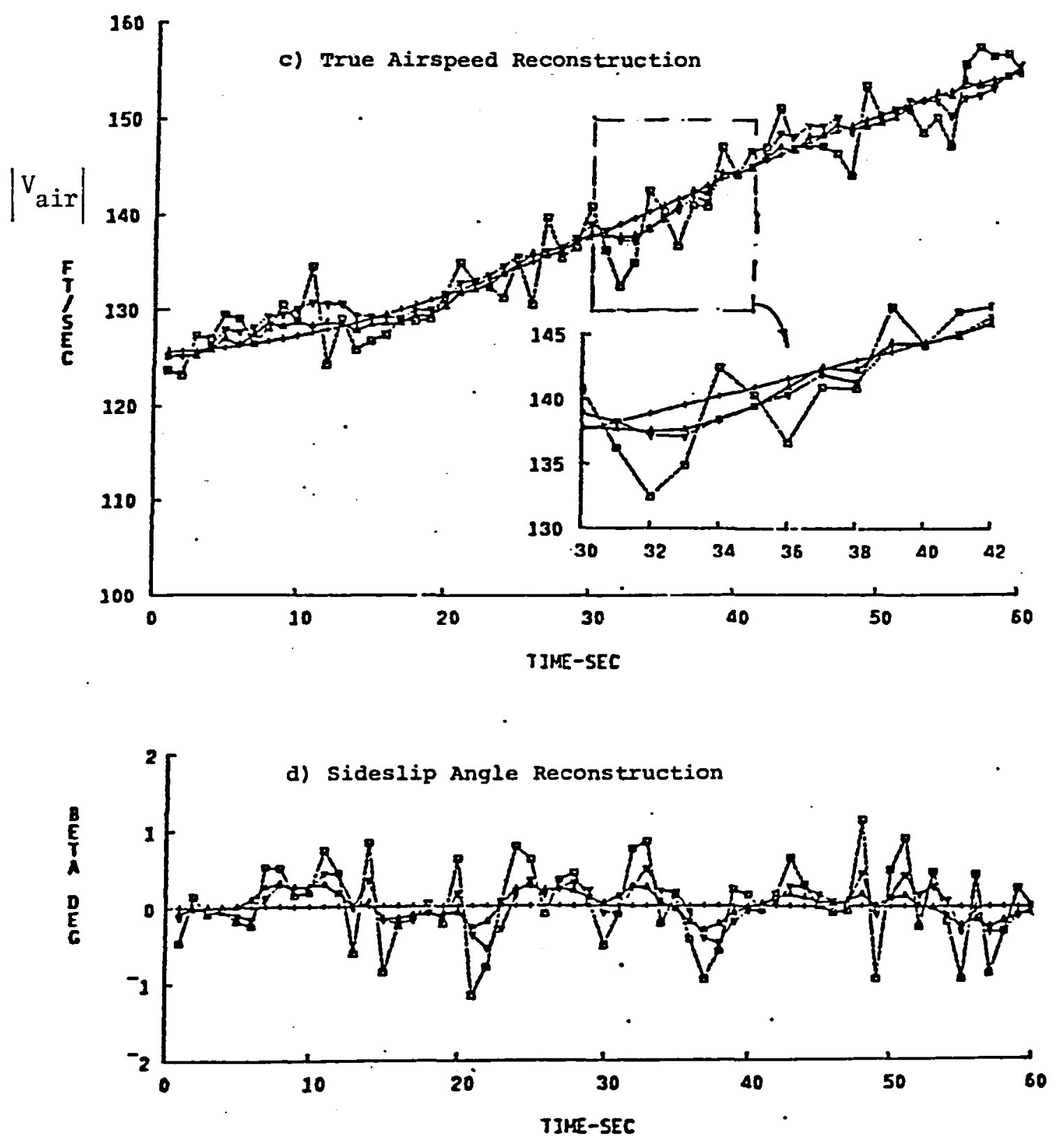

Figure 3.5: Ppplication of the Cptimal Flight Path Reconstruction Algorithm to the Climbing Turn Generic Data (cont'd) 


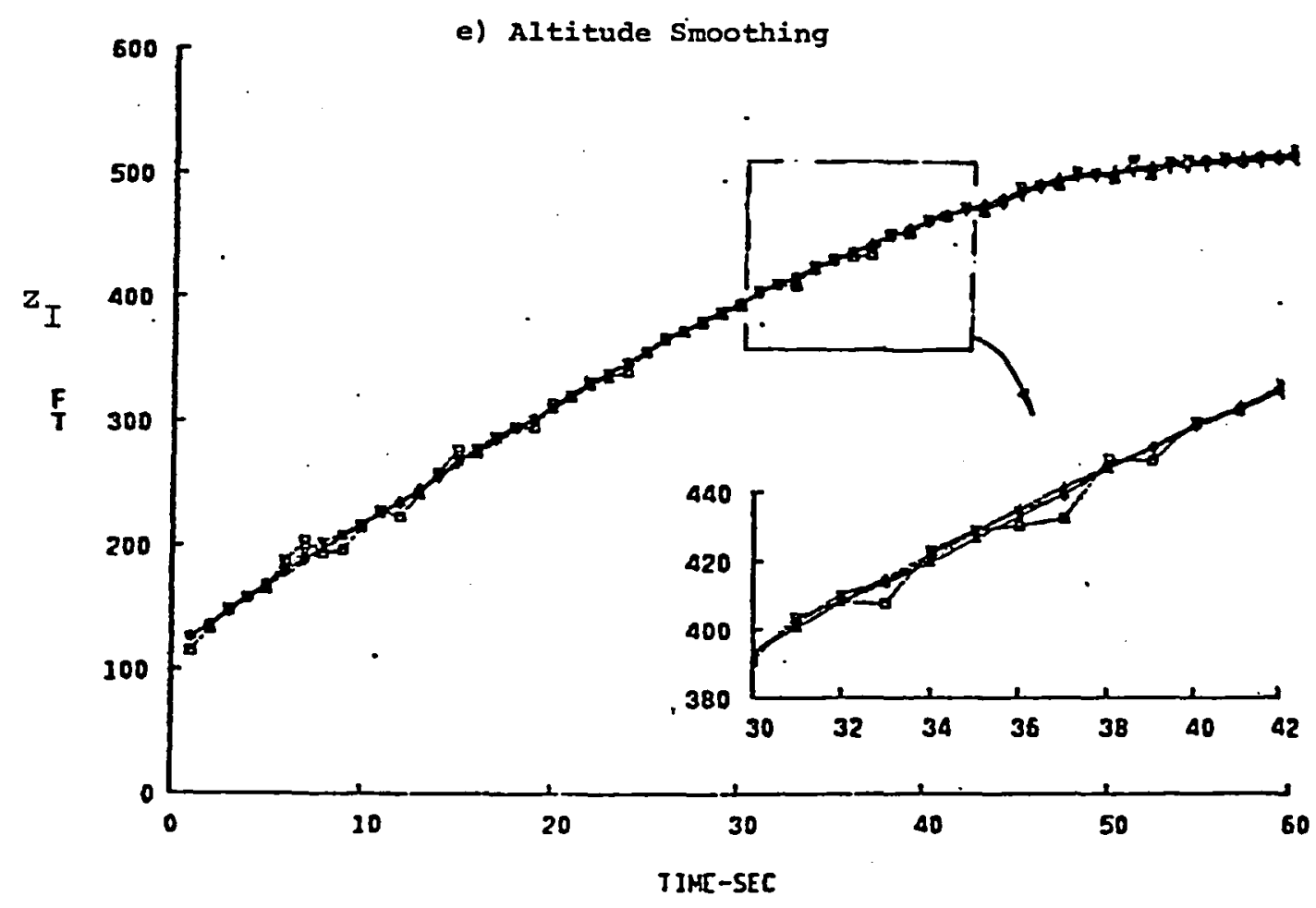

Figure 3.5: Application of the Cptimal Flight Path Feconstruction Algorithm to the climbing Turn Generic Data (cont'd) 

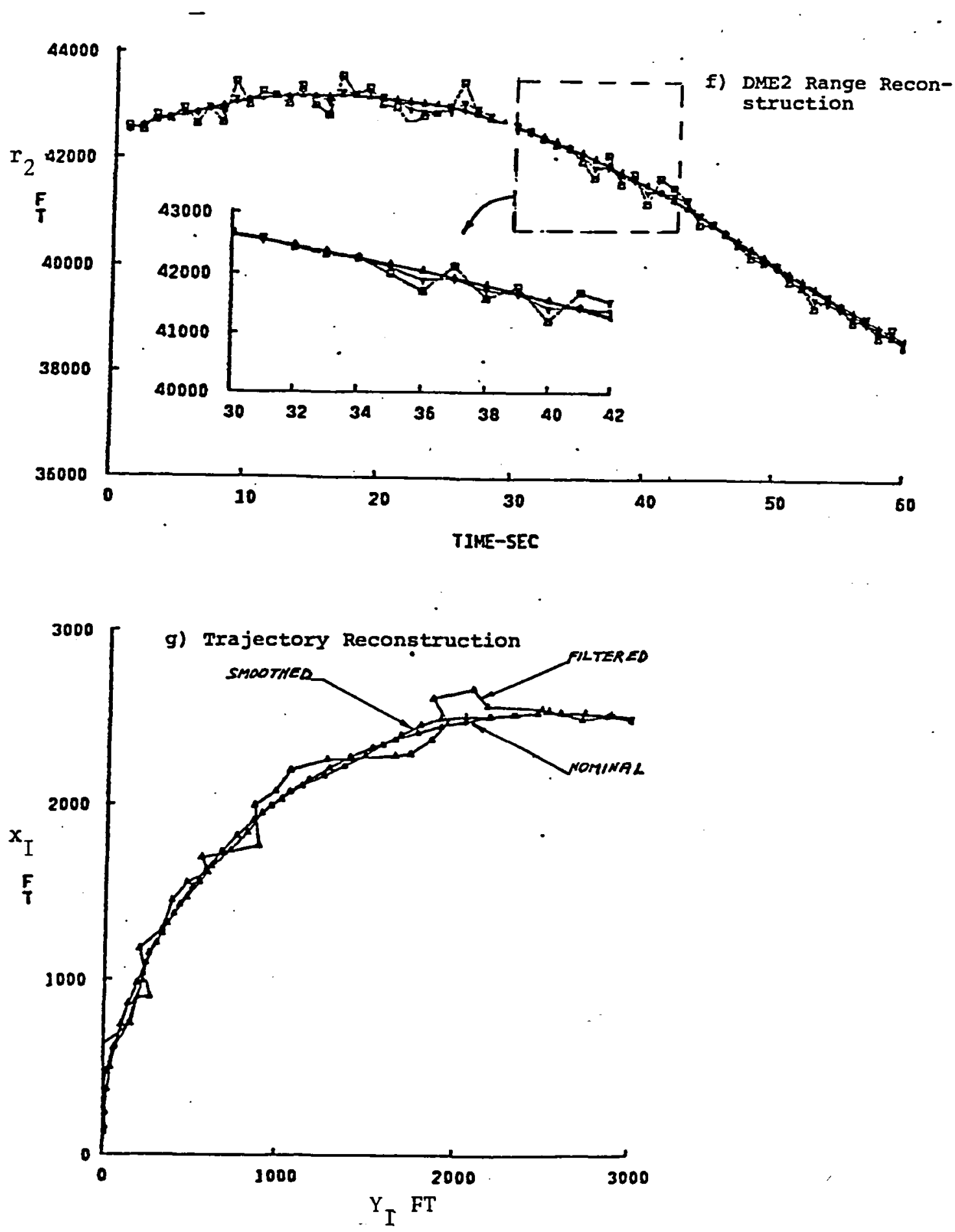

Figure 3.5: Application of the Cptimal Flight Path

Feconstruction f.lgorithm to the Climbing Turn Generic Data (cont'd) 
Examples of the optimal flight path reconstruction algorithm's application to a segment of the flight of September 17 th, 1981 are presented in Fig. 3.6. As these are actual data, the nominal (+) flight path time histories known for the generic simulation, do not appear in the plots. The translational results are presented for the complete model $B$; thus the $x(y)$-trajectory tracings are scaled geographic coordinates $(\lambda, \mu)$ variations with respect to the flight starting point $\left(\lambda_{0}, \mu_{0}\right)$. The meteorological wind information ( 6 knots/50 degrees azimuth) was implemented appropriately. Approximate values for the initial conditions of the state variables were deduced from the preprocessed results. The engaged DME stations for the shown flight segment were Robbinsville and Colts Neck (see Table D.2). As expected following the pilots' report, the flight path variables' time histories reflect strong turbulence conditions. This is particularly evident as entering a holding pattern the aircraft has been turning in this flight segment. Results of the processing of the mission data of September 17 th, 1981 verify the SPIFR flight path reconstruction concept.

With regard to the reconstruction of the trajectory horizontal projection, note that the performance of the DME/DME scheme is inherently limited by the GDOP effect. As elaborated in Chapter 2, the SPIFR mission planning minimized 
this adverse effect intentionally. Thus, the accuracy of position estimation, achieved in our experiments is more than adequate for allocation of the flight segments, for performance indicators computations. For more accurate position estimation the multiple DME scheme (with redundant measurements) should be employed. This will reduce significantly the sensitivity both to the GDOP, to DME biases and to the wind effects. To demonstrate the potential position accuracy attainable, when going to multiple (redundant) DME realization, a DME/DME/DME case study is explored analytically in Appendix C.3. It is shown that improvement up to 308 in position accuracy with respect to the results in Table 3.2 may be achieved. 
a) PITCH ANGLE SMOOTHING (SEPT, 17, 1981)

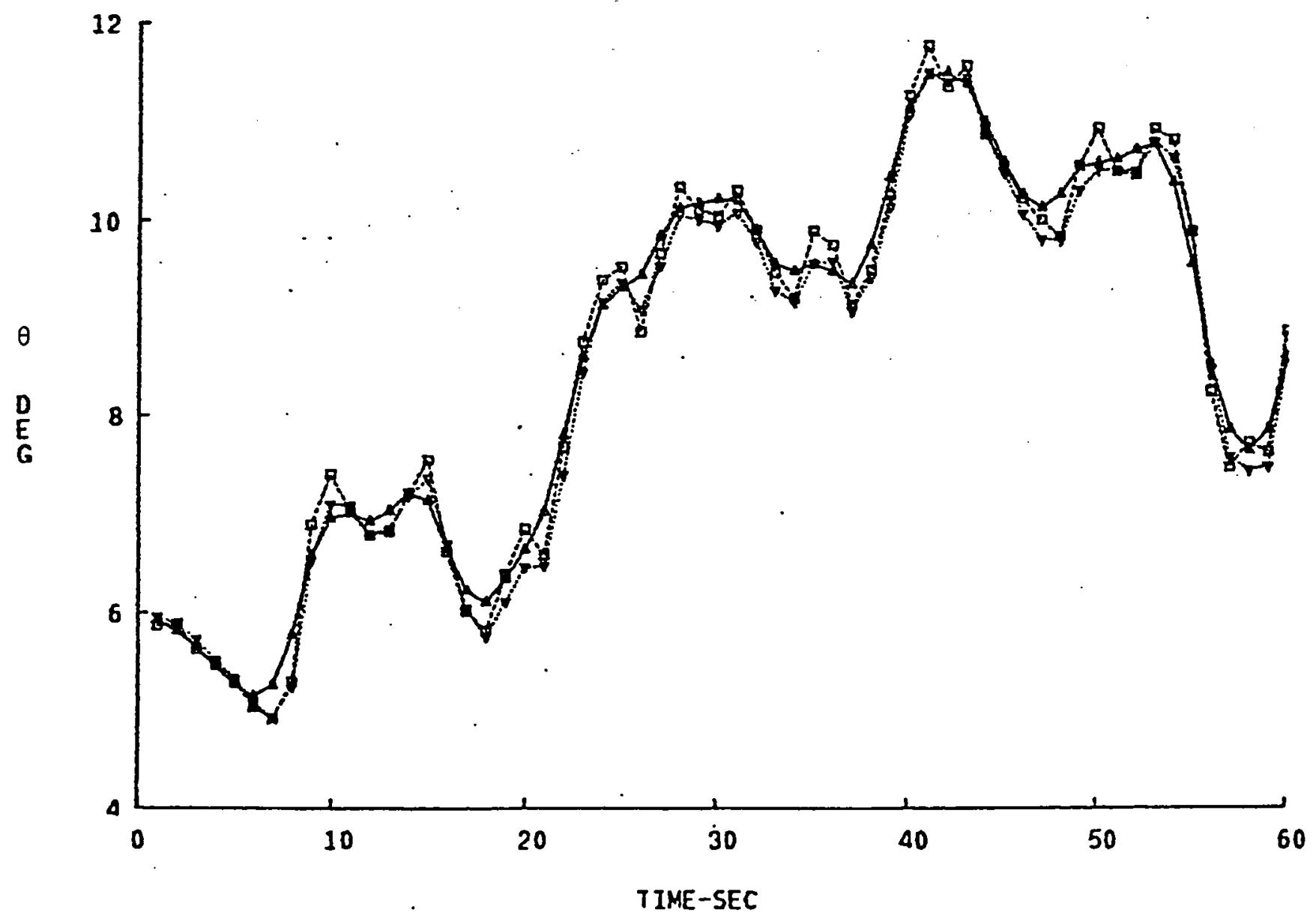

Figure 3.6: Application of the Optimal Flight Path

Reconstruction Algorithm to Actual Flight-Test Data 
b) YAW RATE SMOOTHING (SEPT. 17, 1981)

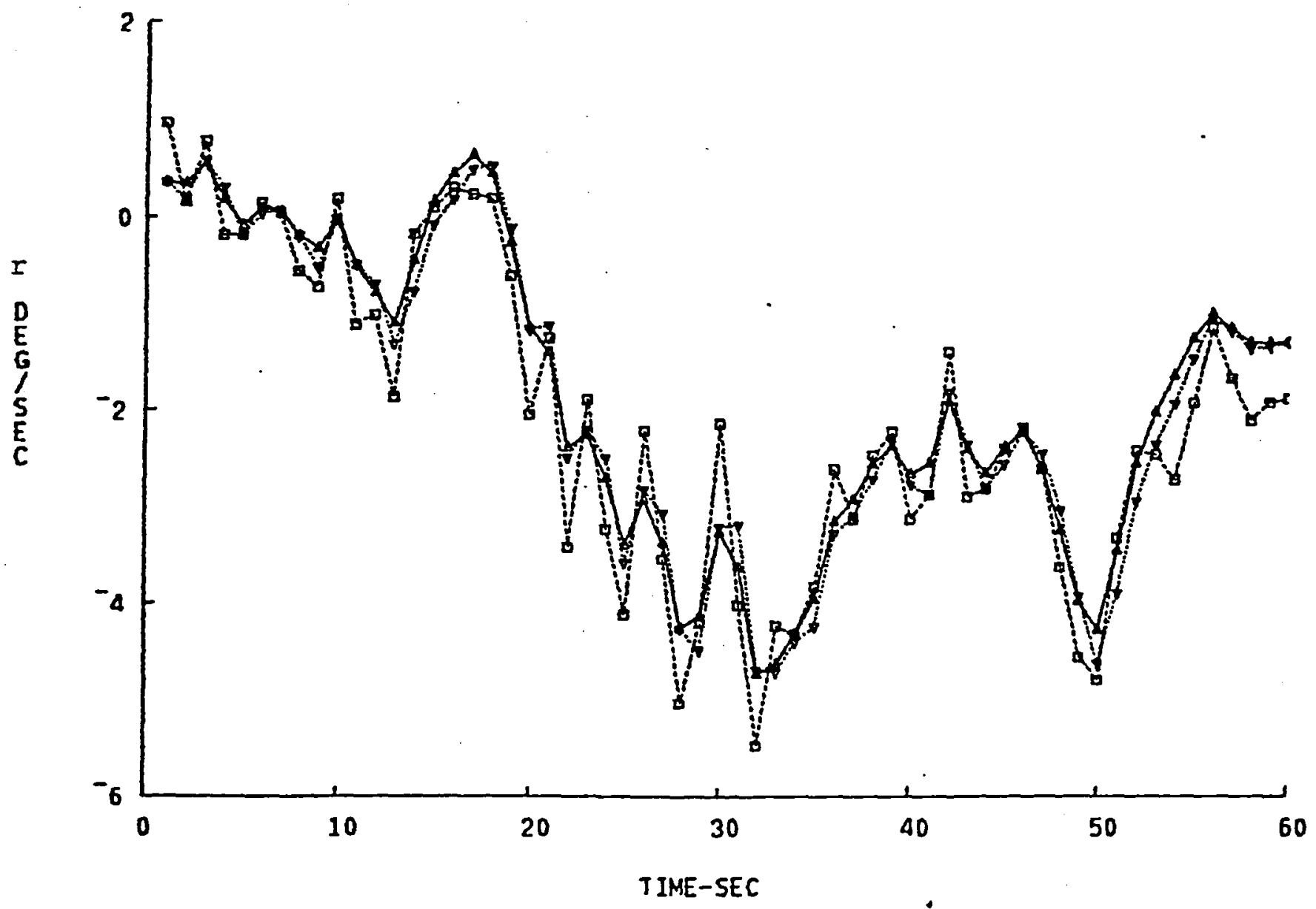

Figure 3.6: Application of the Cptimal Flight Path Reconstruction Algorithm to fictual Flight-Test Datà (cont'd) 
c) TRUE AIRSPEED RECONSTRUCTION (SEPT. 17, 1981)

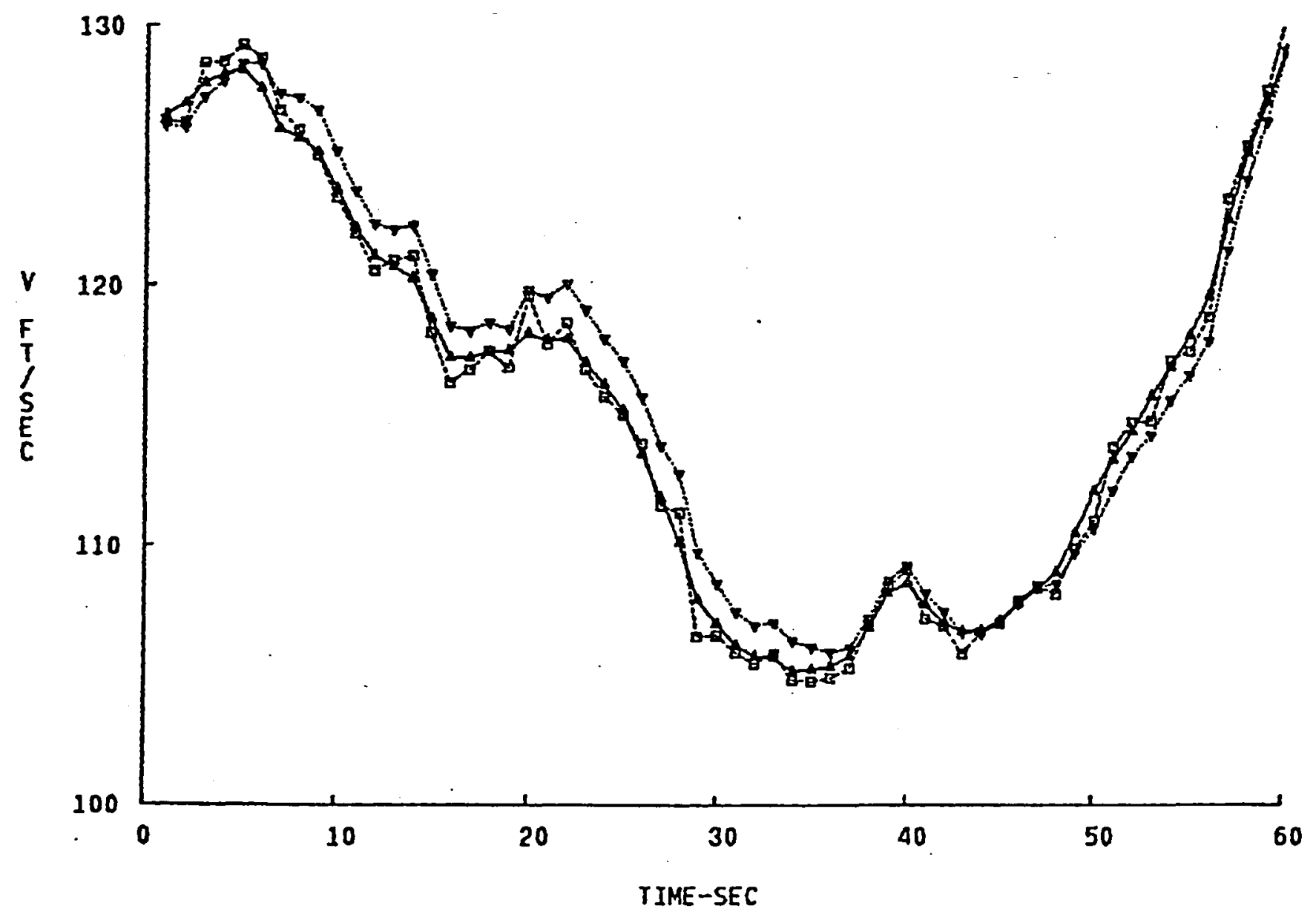

Figure 3.6: Application of the Cptimal Flight Path Reconstruction Algorithm to factual Flight-Test Datá (cont'd) 
d) ANGLE-OF-ATTACK RECONSTRUCTION (SEPT. 17, 198I)

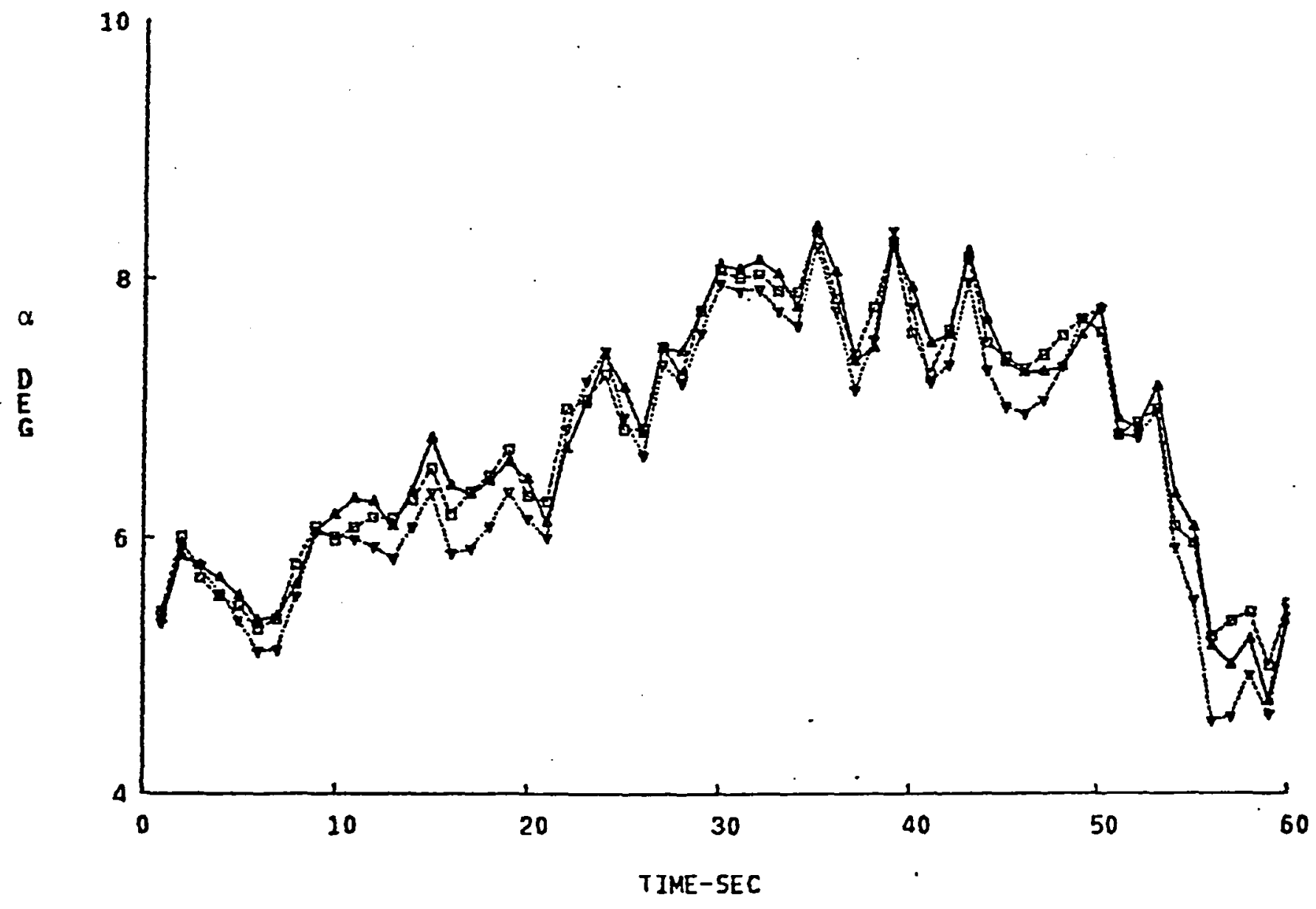

Figure 3.6: Application of the Cptimal Flight Path Reconstruction Algorithm to fictual Flight-Test Datá (cont'd) 
e) ALTITUDE RECONSTRUCTION (SEPT. 17, 1981)

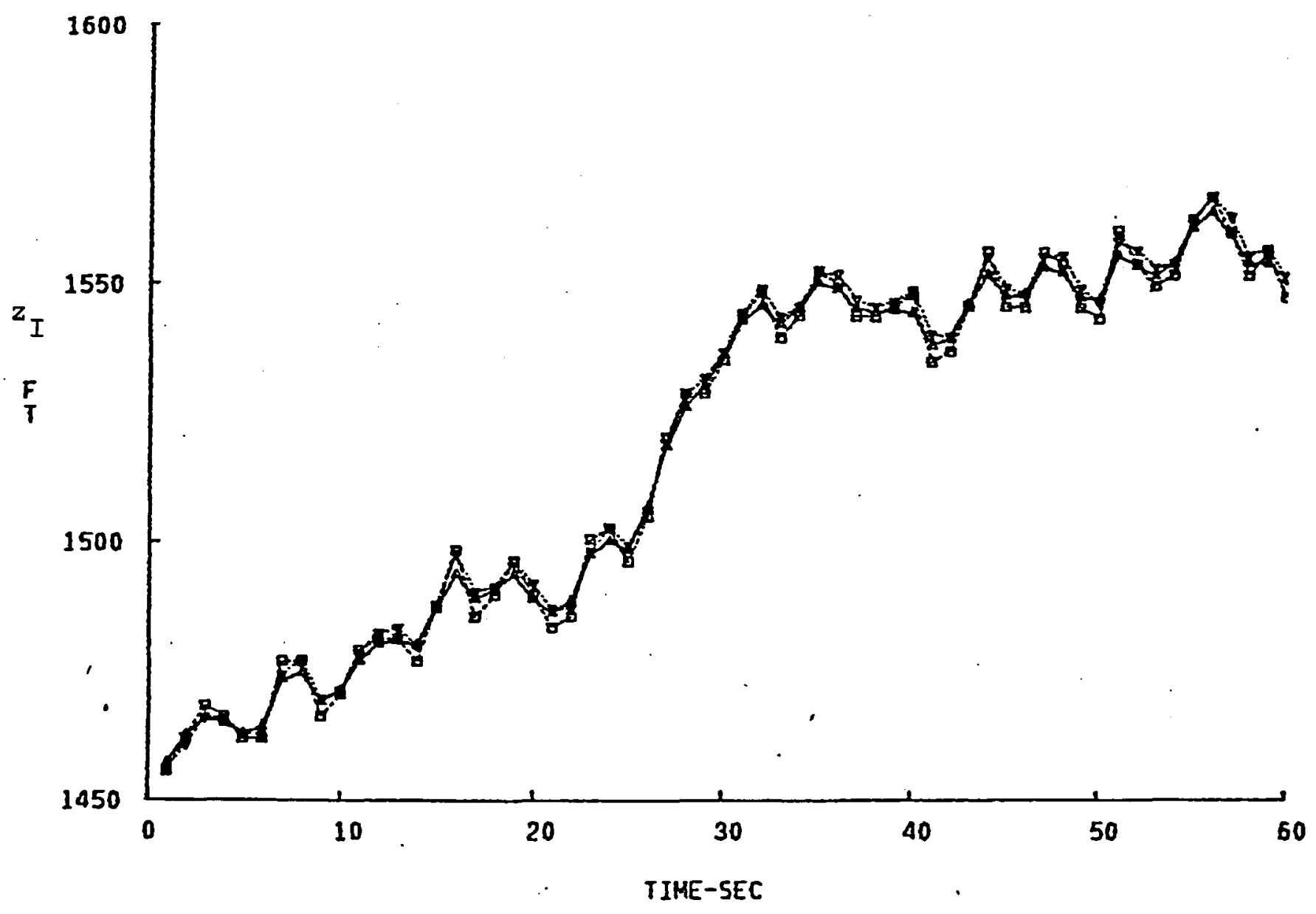

Figure 3.6: Application of the Cptimal Flight Path

Reconstruction Algorithm to fictual Flight-Test Datá (cont'd) 
f) TRAJECTORY RECONSTRUCTION (SEPT. 17, 1981)

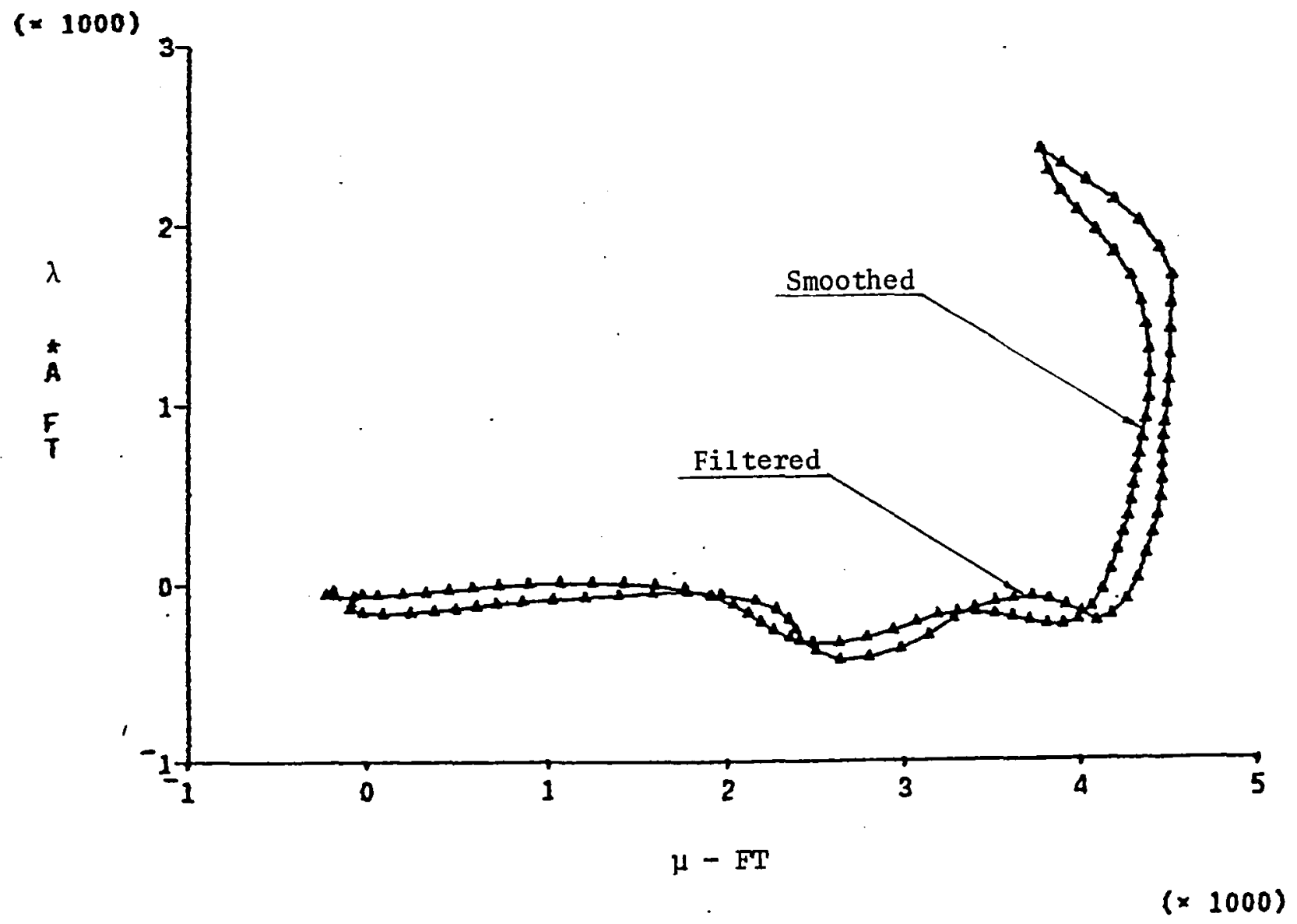

Figure 3.6: Application of the Cptimal Flight Path Reconstruction Algorithm to fictual Flight-iest Data (cont'd) 
As an additional concept verification, a closer look was taken at the mission starting point $\left(\lambda_{0}, \mu_{0}\right)$ for the first eleven SPIFR flights, which was defined by an intersection of two VOR radials under ideal GDOY conditions. In all these missions the same IFR-experienced test pilot performed the navigation to this starting point.

The coordinates of the starting point were obtained for each flight using the flight path reconstruction algorithm with DME range measurements to the same navigational stations. The dispersion of the points $\left(\lambda_{\circ i}, \mu_{o i}\right)$ is shown in Fig. 3.7. The standard deviations are,

$$
\left.\begin{array}{rl}
\sigma_{a \lambda}=1700 \mathrm{ft} & \\
\sigma_{\text {aucos }}=2850 \mathrm{ft} & \text { (along-track position estimation } \\
& \text { is more affected by delays in } \\
& \text { data acquisition system triggering) }
\end{array}\right\}
$$

Note that with regard to the flight path reconstruction algorithm, the computation of the points $\left(\lambda_{\circ i}, \mu_{\circ i}\right)$ constitutes a static estimation $(t=0)$, whose accuracy based on eq. (2-97) is,

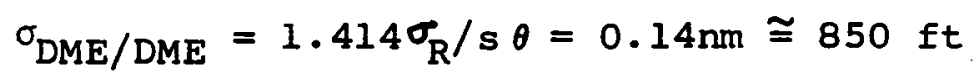

as determined from Fig. 3.8 for $\theta=90 \mathrm{deg}$. From eq. (2-96) and Fig. 3.8, the VOR/VOR error is, 


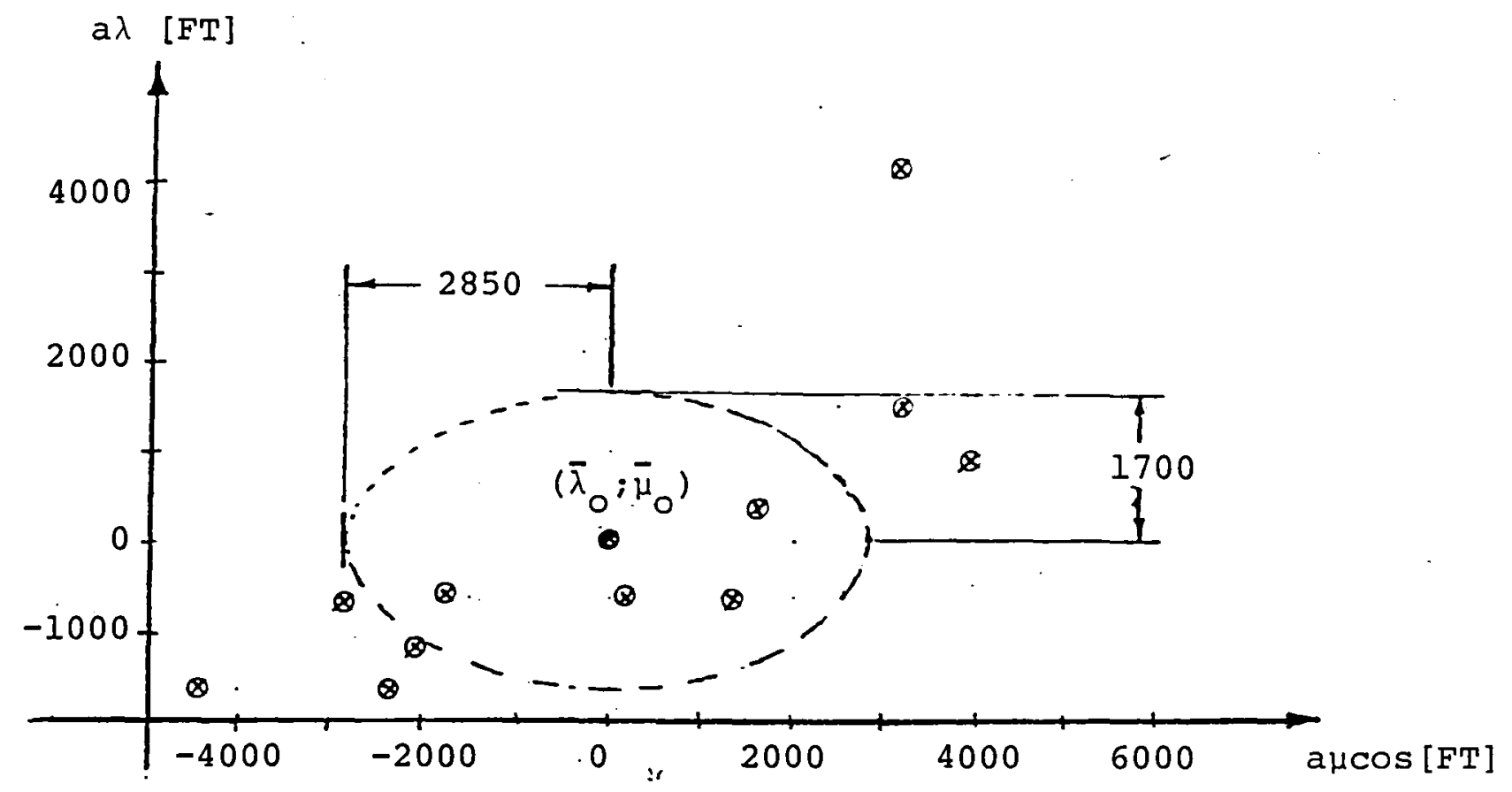

Figure 3.7: Starting Point Dispersion for the First Eleven SPIFR Tasks

$$
{ }^{\mathrm{VOR} / \mathrm{VOR}}=1.414 \times 19 \times 1.9 \times 0.01745 / 1.414 \cong 0.63 \mathrm{~nm} \cong 3830 \mathrm{ft}(3-70)
$$

These results indicate that the VOR/VOR system onboard the ARA was functioning within specifications (eq. (2-98)).

To conclude this chapter, several samples of optimal trajectory reconstruction results (horizontal projections) for complete SPIFR missions are presented in Figures 3.9 to 3.11. The plus symbols in the figures provide the time di- 


$$
1^{N}
$$

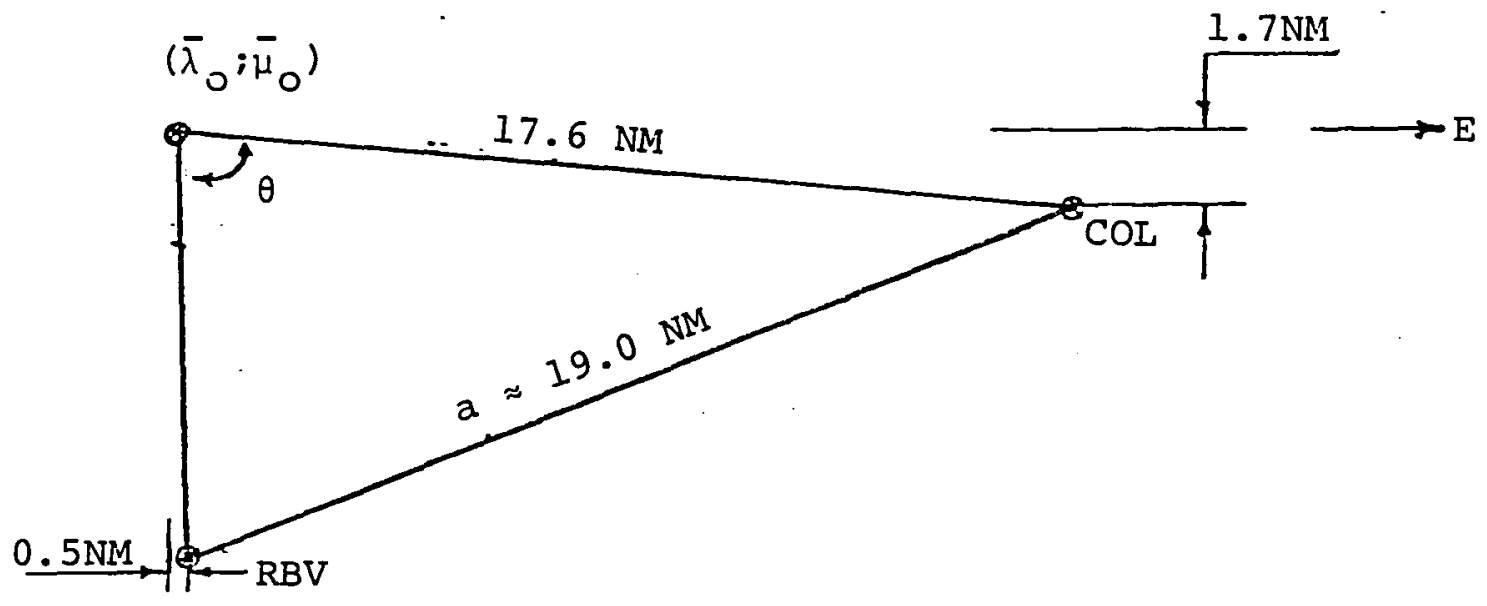

Figure 3.8: Aircraft-Navigational Stations Geometry for the Starting Point for the First Eleven SPIFR Missions

mension; they are $50 \mathrm{sec}$ apart. Comparison of these figures to Figures 2.5 to 2.7 shows that due to the minimum-GDOP design, the actual navigational tracks, although flown with the less accurate VOR/VOR mode, did not diverge significantly from the respective planned variants. The accuracy of flight path reconstruction is demonstrated in these plots by the alignment of the trajectory starting point with the 
glide slope or the runway direction. Recall that the data acquisition system was being initiated over Forrestal airfield reference point; also, the $x-y$ axes set is the scaled North-East (latitude-longitude) navigational grid. As pointed out in Chapter 2, different altitude and airspeed profiles have been planned for each track variant. A sample of such profiles is presented in Fig. 3.12 (again: the "+" and " $x$ " are $50 \mathrm{sec}$ apart). The time histories in this figure are results of optimal smoothing processing and the impression of high-frequencies content in the airspeed variation is due to the large time span (over $30 \mathrm{~min}$ ), which is required to simulate a realistic SPIFR mission. Time histories of this type are used in the next chapter to find out how well could the pilot hold altitude or airspeed with a given configuration. 


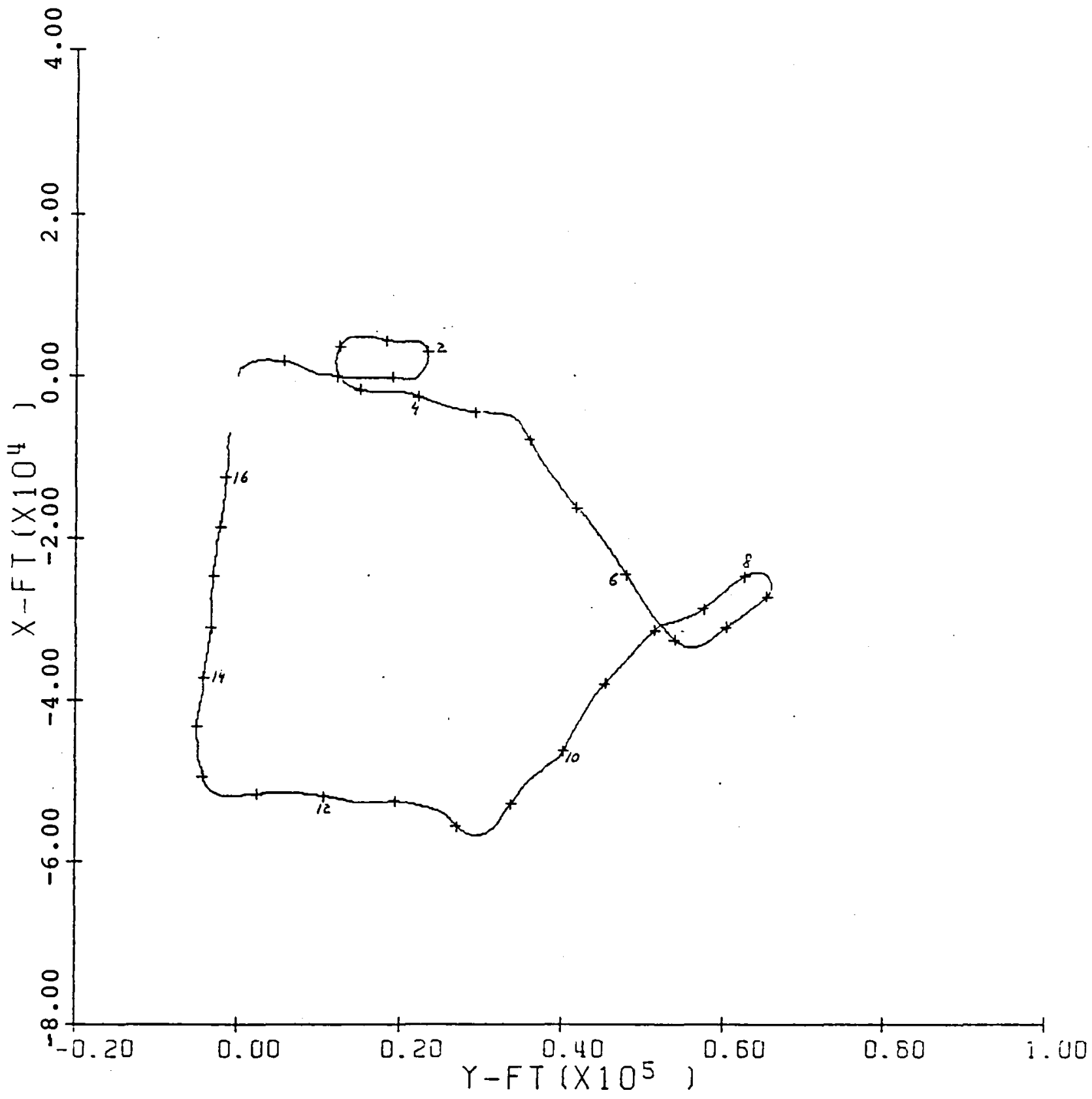

$\begin{array}{llllll}\text { FLIGHT } & 5 & \text { OAY } & 4 & \text { MONTH } & 5 \text { YEAR } \\ \text { CONFIGURATION } & 4 & \text { PILOT } & 1982\end{array}$

Figure 3.9: Reconstruction of a Navigational Trajectory Second Variant 


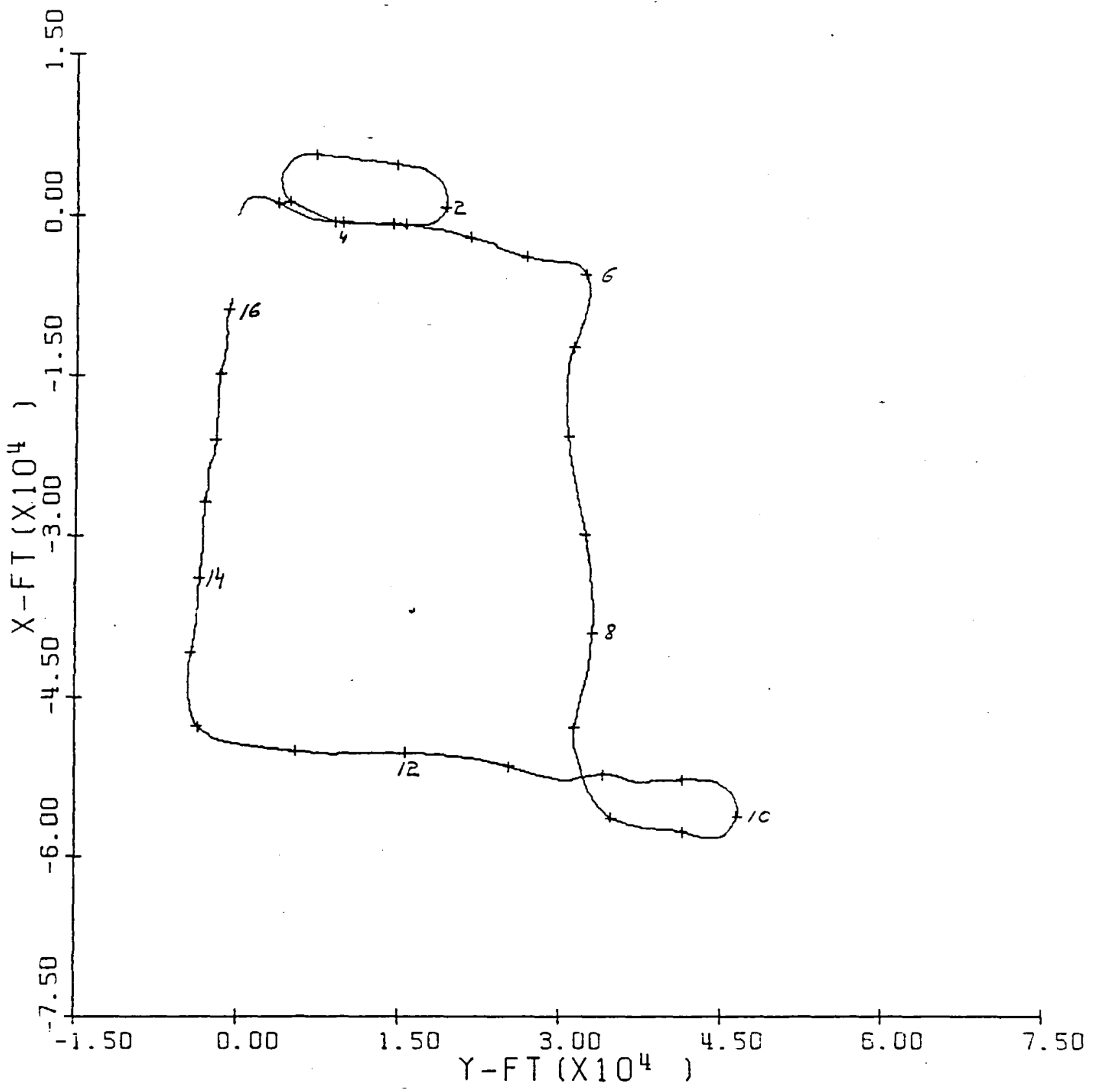

$\begin{array}{lcllll}\text { FLIGHT } & 5 & \text { DAY } & 7 & \text { MONTH } & 5 \text { YEAR } \\ \text { CONFIGURATION } & 9 & \text { PILOT } & 1982 \\ \text { TRACK } & 3\end{array}$

Figure 3.10: Reconstruction of a Navigational Trajectory Third Variant 


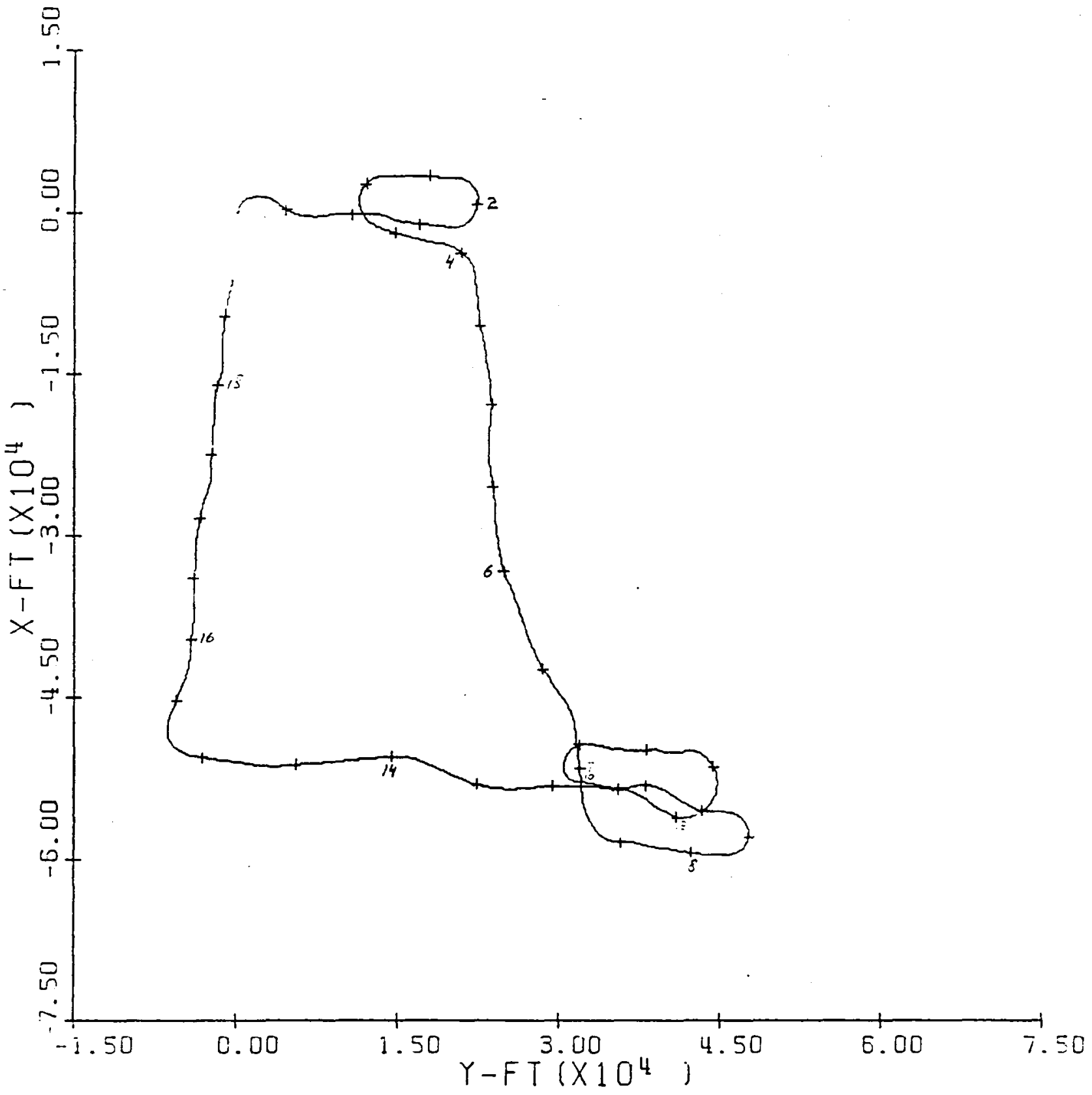

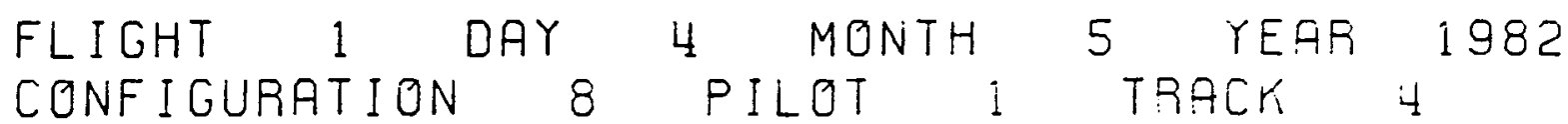

Figure 3.11: Reconstruction of a Navigational Trajectory Fourth Variant 


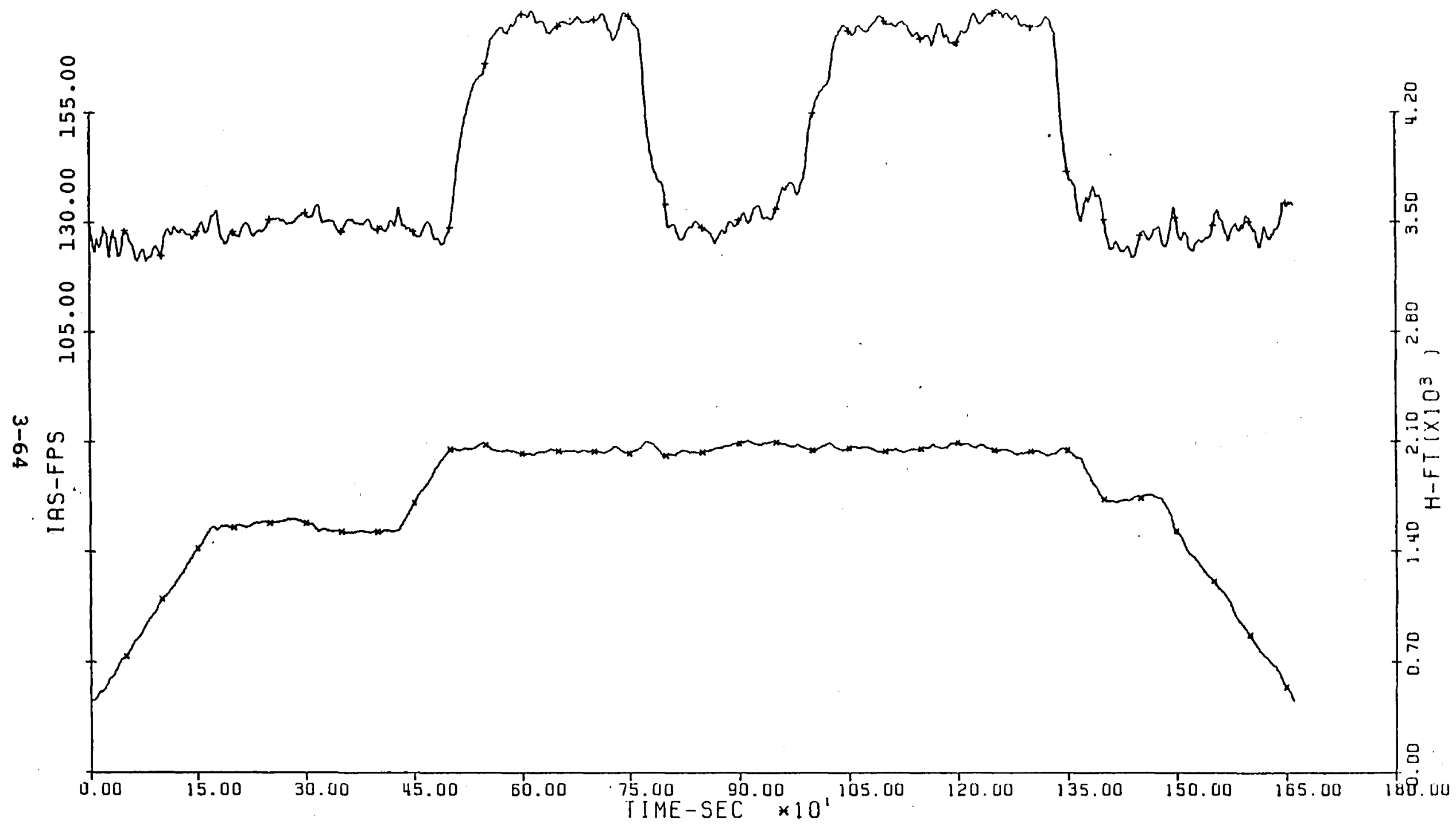

$\begin{array}{llllll}\text { FLIGHT } & 4 & \text { DAY } & 7 \text { MONTH } & 5 \text { YEAR } & 1982 \\ \text { CONFIGURATION } & 10 & \text { PILOT } 1 & \text { TRACK } & 2\end{array}$

Figure 3.12: Reconstruction of Airspeed and Altitude Profiles for a Complete SPIFR Task 


\section{Chapter 4}

\section{STATISTICAL ANALYSIS AND DERIVATION OF FLYING QUALITIES CRITERIA FOR SPIFR OPERATIONS}

The message in Fig. 4.1 is that Chapter 4 constitutes a focal point of the SPIFR program. It uses as "inputs" the "outputs" of Chapters 2 and 3, and applies statistical processing to these "inputs". As a result, the first objective of this research, which is deduction of flying qualities criteria for SPIFR operations, is achieved.

Section 4.1 elaborates on the principles of the multiple regression analysis. A detailed discussion of the analytically-derived candidate criteria was given in Chapter 2 . They may be reflected in "subjective" Pilot Opinion Ratings (PORs) and "objective" performance indicators, which were computed in Section 4.2 (using the algorithm of Chapter 3 ). Results of actual implementation of the stepwise multivariate regression algorithm to the SPIFR data-base are presented in Section 4.3. 


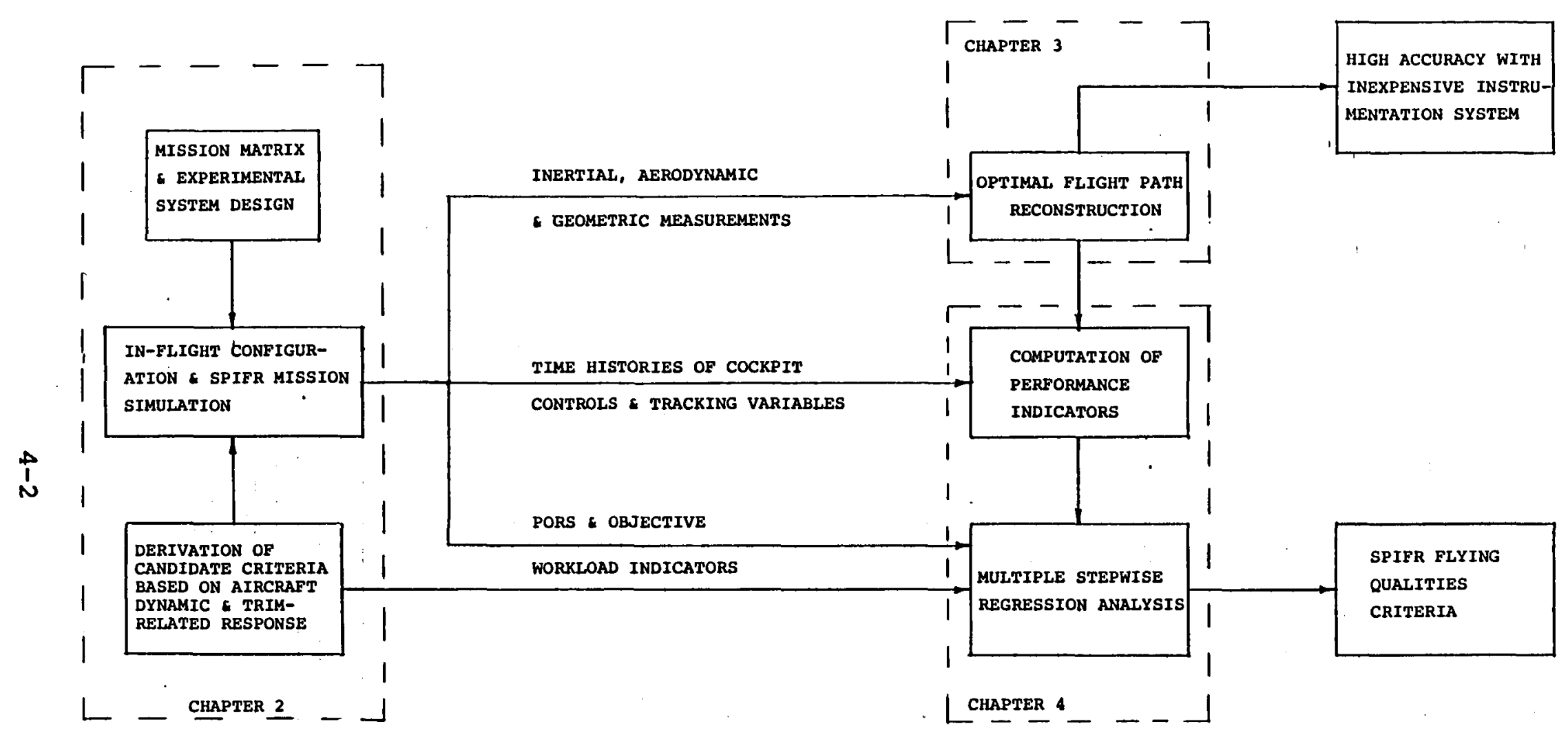

Figure 4.1: SPIFR Research Flow Chart 


\subsection{THE MULTIPLE REGRESSION ALGORITHM}

In this section the multiple regression analysis, which is used to deduce the SPIFR flying qualities criteria, is discussed. The discussion is based on the excellent statistical text of Ref. 59 .

Several flight tests were performed with each of the $n$ chosen aerodynamic configurations. As shown in Chapter 2, each configuration may be characterized by various candidate criteria. Following an extensive theoretical study $p$ such candidate criteria were chosen. The purpose of the regression analysis was to single out statistically significant SPIFR flying qualities criteria from the candidate criteria "pool". This may be done by evaluating the latter versus performance indicators, which constitute the experimental results. Such statistical evaluation of each performance indicator vector against the "pool" of the candidate criteria is referred to as multiple regression.

The target relationship between a performance indicator vector, $Y$, and the candidate criteria matrix, $[x]$, is:

$$
\underline{\underline{Y}}=[\mathrm{x}] \underline{\underline{\beta}}+\underline{\mathrm{a}}
$$

where the experiment-based vector $Y$ is, 


$$
\underline{\underline{y}}^{\mathrm{T}}=\left[\mathrm{y}_{1} \mathrm{y}_{2} \ldots \cdots \mathrm{y}_{\mathrm{n}}\right]^{\mathrm{T}}
$$

The vector of partial regression coefficients, $\underline{\beta}$, is:

$$
\underline{\beta}^{T}=\left[\begin{array}{lllll}
\beta_{0} & \beta_{1} & \beta_{2} & \ldots & \ldots \\
\beta_{n}
\end{array}\right]^{T}
$$

The regression error vector, 뜨, is:

$$
\underline{\underline{u}}^{T}=\left[u_{1} u_{2} \ldots \ldots u_{n}\right]^{T}
$$

In the following definition

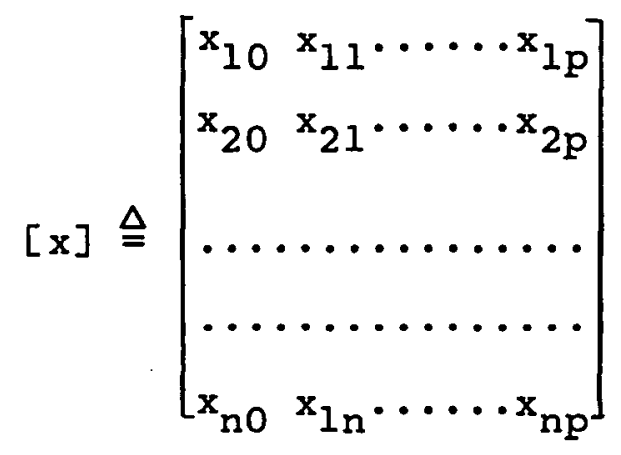

the columns of matrix [x] (excluding the first column), or the candidate criteria vectors $\underline{x}_{1}$ to $\underline{x}_{p}$, consist of the numerical values for each configuration, with $x_{i 0}=1$ for all i. The length of these vectors, $n$, is equal to the length of the vector of indicators $\underline{\underline{y}}$. If one data point $y_{i}$ is available for each configuration, then $\mathrm{n}$ is also the number of configurations considered. Due to configuration replications or to several segments of same nature in one flight, 
there may be different numbers of data points for different configurations. In such a case $\mathrm{n}$ can be larger than the number of in-flight-simulated configurations. Note also that there may be another reason for repeated values in a column of $[x]$, due to the fact that a configuration change does not affect a given criterion. For example, a change in $M_{\delta_{t}}$ does not affect the total damping coefficient and it will remain identical to the nominal value. Assumption that the components of a are random quantities, independently distributed with zero mean and constant variance $\sigma^{2}$ (Gaussian probability distribution), renders:

$$
E(\underline{a})=0
$$

$$
\operatorname{Var}(\underline{\mathrm{u}})=\mathrm{E}\left(\underline{\underline{\mathrm{uu}}}^{\mathrm{T}}\right)=\sigma^{2}\left[\mathrm{I}_{\mathrm{n}}\right]
$$

Equations (4-1), (4-6) and (4-7) yield the vector of the expected values of the observations or of a given performance indicator,

$$
E(\underline{y})=[x] \underline{\beta}
$$

By minimizing the sum of squared deviations of the observations from their expected value, the least squares estimator of $\underline{\beta}, \underline{b}$ is obtained,

$$
\underline{b}=\left([x]^{T}[x]\right)^{-1}[x]^{T} y
$$


$b_{0}$ is usually referred to as the intercept. A vector of fitted (or predicted) values $y$ may be now defined as,

$$
\underline{y}=[x] \underline{b}
$$

The vector of the observed residuals $e$, is then given by,

$$
\underline{e}=\underline{y}-\hat{q}=\underline{y}-[x] \underline{b}
$$

The percentage of variation explained by the model is given by the square of the multiple correlation coefficient $\mathrm{R}^{2}$,

$$
R^{2}=\left[1-\bar{\Sigma}\left(y_{i}-y_{i}\right)^{2} / \bar{\Sigma}\left(y_{i}-\bar{y}\right)^{2}\right] 100 z
$$

where

$$
\bar{y}=\Sigma y_{i} / n
$$

Note that this discussion is not restricted to linear relationships in the simplistic sense. Following a first look at the data in a scatter plot, one may decide, for example, that a performance indicator is a linear function of the square root of the fourth candidate criterion. This approach is referred to as "transformation of variables" and can be practiced, exercising care with the underlying analysis assumptions.

A way to verify the regression analysis results is by hypothesis testing. The most commonly investigated hypothesis starts out assuming that all regression coefficients (except 
for the intercept $\beta_{0}$ ) are zero. This implies that there is no linear relationship between the dependent variable and the set of independent variables. The test involves a comparison of the goodness of fit that is obtained when using the full model with the data, to the goodness of fit that results using the $\beta_{i}=0$ (i>1) assumption specified by the null hypothesis. If this assumption gives as good a fit as the full model, then the null hypothesis is not rejected. As the first step of the procedure, the sum of squares due to error associated with the full model by SSE(FM), is written as,

$$
\operatorname{SSE}(F M)=\bar{\Sigma}\left(y_{i}-\hat{y}_{i}\right)^{2} .
$$

SSE(FM) represents the lack of fit in the data associated with the model. The lack of fit in the data which is associated with the null hypothesis assumption is denoted by SSE (RM) ,

$$
\operatorname{SSE}(\mathrm{RM})=\bar{\Sigma}\left(\mathrm{y}_{i}-\bar{y}\right)^{2}
$$

The ratio $\mathrm{F}$,

$$
F=[\operatorname{SSE}(R M)-\operatorname{SSE}(F M)](n-p-1) / \operatorname{SSE}(F M) / p
$$

has the $F$ statisical distribution with $p$ and $(n-p-1)$ degrees of freedom. If the observed $F$ value is large, in comparison to the tabulated value of $F$ with $p$ and $(n-p-1)$ degrees of freedom at the $100 \alpha$ percent level, the result is 
significant at level $\alpha$; that is, the null hypothesis is rejected.

To rank the SPIFR flying qualities criteria among the candidate criteria, a stepwise procedure is employed. It has the feature that the variables are introduced or deleted from the equation one at a time, and it involves examining only a subset of all possible equations. One of the categories into which the stepwise procedure can be classified is the forward selection (FS) method. It starts out assuming that the model constitutes only of the intercept term. The first variable included in the equation is the one which has the highest simple correlation with the dependent variable $y$ - If the regression coefficient of this variable is significantly different from zero, it is retained in the equation, and a search for a second variable is made. The variable that enters the equation as the second variable is the one which has the highest correlation with $y$, after $y$ has been adjusted for the effect of the first variable. The significance of the regression coefficient of the second variable is then tested. If the regression coefficient is significant, a search for a third variable is made in the same way. The procedure is terminated when the last variable entering the equation has an insignificant regression coefficient or all the variables are included in the equation. An improvement to the FS algorithm which considers 
the possibility of deleting a variable at each stage can be employed: a variable that entered in the earlier stages of selection may be eliminated at later stages. This improvement is referred to (Ref. 60) as backward elimination (BE).

Efficient FORTRAN coding of the stepwise multiple regression algorithm is available in Ref. 53. It provides the capability of separately defining the significance levels $\alpha$ for entering and deleting variables. For the SPIFR analysis $\alpha=0.05$ was chosen for both tests.

\subsection{THE FLIGHT-TEST-BASED PERFORMANCE INDICATORS}

"Performance indicators" are the various metrics that may reflect the effects of SPIFR configuration changes. Using the nomenclature of section 4.1 , they constitute the possibilities for the vector of dependent variables for the regression analysis, $Y$. The performance indicators may be classified into two distinct categories: (1) the subjective Pilot Opinion Ratings (PORs), and (2) the objective system evaluations.

As the human operator is an integral part of the control and guidance loop, the subjective PORs constitute important experimental results. The pilot flying a realistic SPIFR 
mission may pass direct judgement with regard to the effect of a given configuration change. One scale that relates pilot's opinions about the ease or difficulty with which airplanes can be controlled in a given flight situation to a numerical rating, is the Cooper-Harper Rating (CHR) 10-point scale (Ref. 61). It has been widely accepted as an aircraft performance metric. Several investigators advocate a separate metric for the assessment of pilot workload. The Simpson-Sheridan 10-point workload Rating (SSR) is an example of this approach (Ref. 62). Other researchers (as in Ref. 63) think that the CHR scale reflects workload as well as performance levels. To study this problem with regard to the SPIFR flight regime, both the CHR and the SSR scales were used in this program to provide experimental data for comparison. As up to three tasks could were flown consecutively, knee-pad versions of both scales and of the grading sheet were prepared for in-flight pilot debriefing (Fig. 4.2). As obvious from Fig. 4.2c, the pilot was required to evaluate the airplane performance and workload levels along entire SPIFR missions as well as along several segments characteristic to this flight regime. 


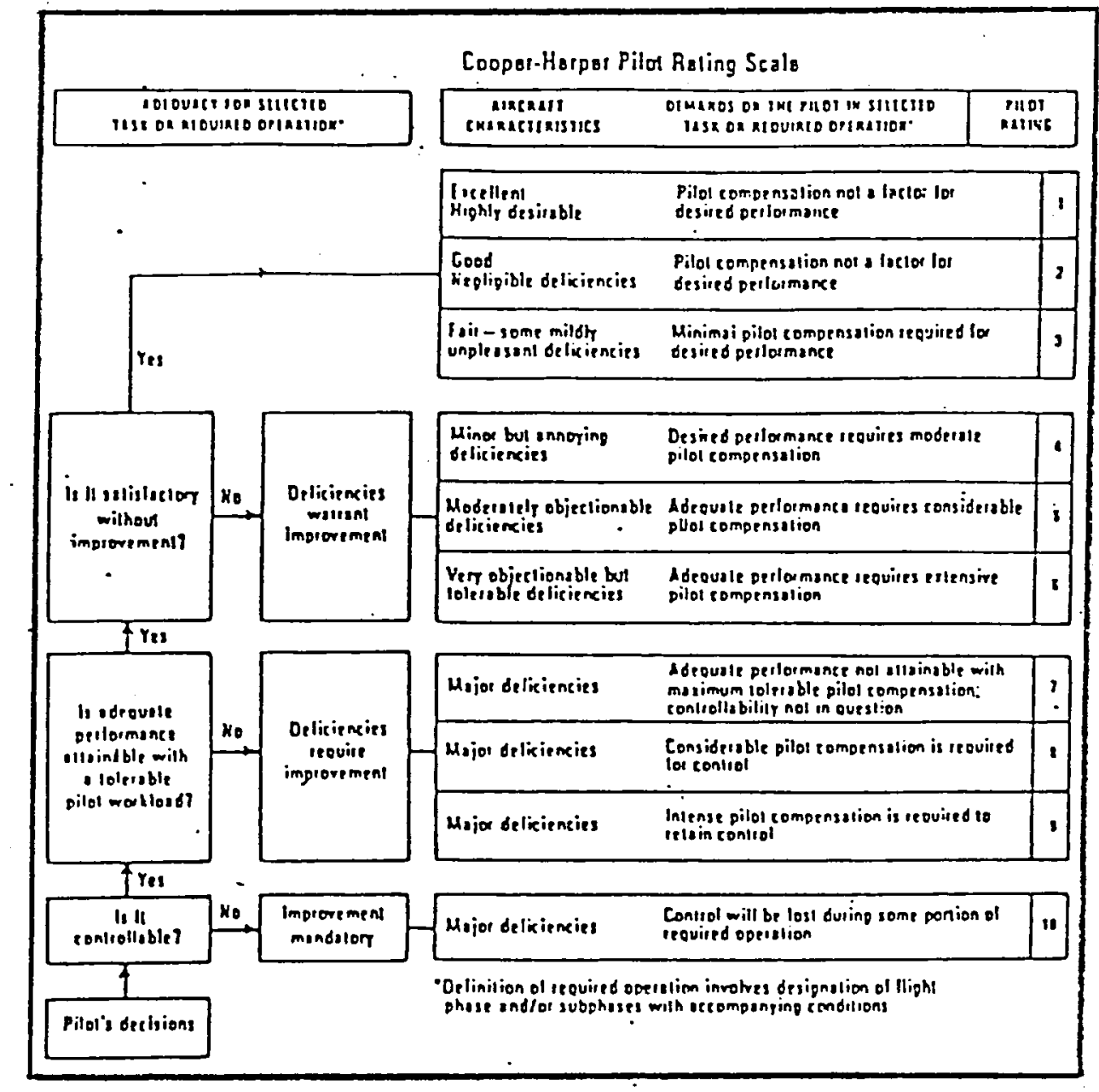

a) Performance CHR Scale

\section{Figure 4.2: Knee-pad Versions of the Performance and Workload PORs and of the Evaluation sheet}




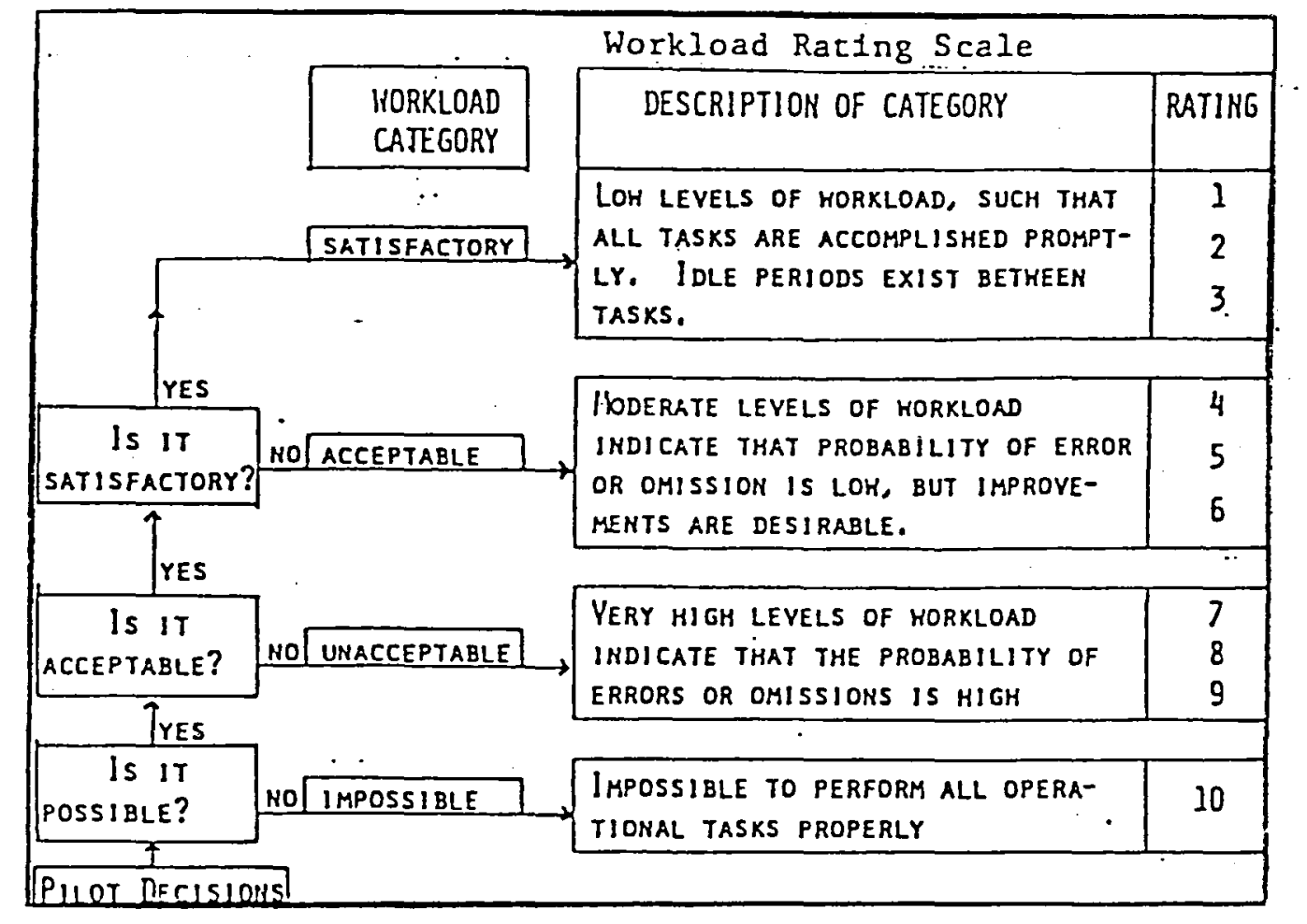

b) Workload ssR Scale

Figure 4.2: Knee-pad Versions of the Performance ana
hiorkload PORs and of the Evaluation sheet (cont'd) 


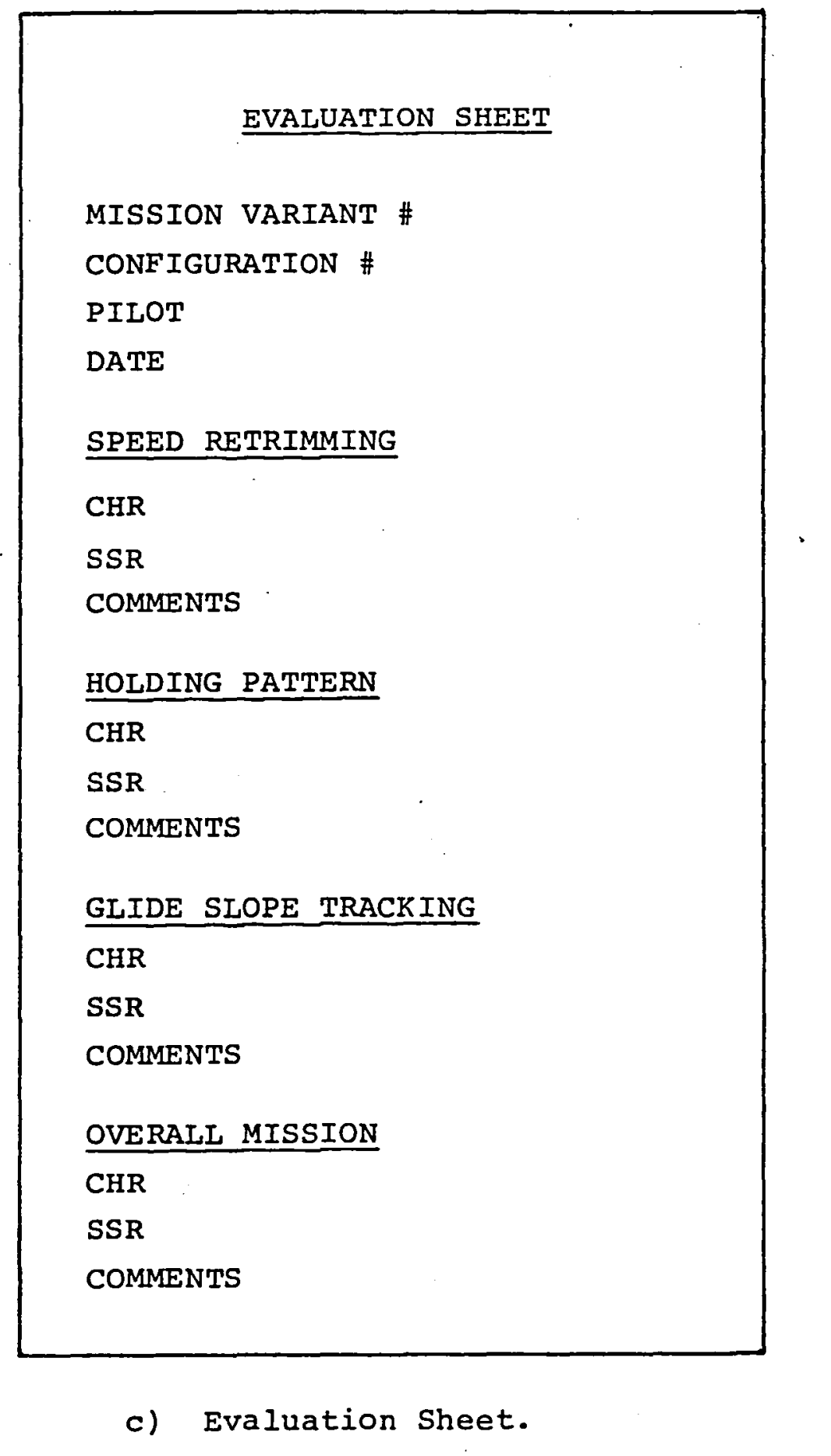

Figure 4.2: Knee-pad Versions of the Performance and Viorkload PORs and of the Evaluation sheet (cont'd)

$$
4-13
$$


To complete this discussion with regard to the subjective performance indicators, there are researchers who not only advocate a separate workload metric, but who attempt to distinguish between audio-visual, mental and motoric components of workload (Ref. 64, 65, 66 and 67). Such a differentiating approach may be advantageous when the objective is, for example, optimal arrangement of cockpit displays.' This author, along with others (as in Ref. 68), prefers the assessment of the overall workload effect, which is most suited to evaluation of a self-contained flight regime.

The flight testing was carried out by the Princeton University Chief Test Pilot only. In the research reported in Ref. 69 it was found that pilots differ by constant shifts, rendering similar gradients and trends of variation. At the time the SPIFR experiments were conducted, the Princeton Flight Reasearch Laboratory Pilot had accumulated over 600 logged IFR hours and total time of about 5000 hours. He flew each of the fifteen configurations twice. As pointed out in Ref. 68, master pilots exhibit excellent repeatibility in their performance, which was confirmed by the results of this program.

Each component of the column vectors of the performance indicators presented in this section, is a result of averaging individual indicator values, which were obtained for 
several segments of several SPIFR tasks flown with a given configuration. The subjective Pilot Opinion Ratings (PORs) constitute the major part of the data in Table $4.1 .^{1}$ The two last columns of the table represent an attempt to quantify the pilot's workload under SPIFR conditions objectively. As detailed in Appendix A, the evaluation pilot was asked to extinguish lights turned on (pseudo-randomly) by the microprocessor program. The prerogative for his reaction was that it should take place only if his primary task of flying the SPIFR mission is not affected; pilot's priorities being: (1)control, (2)navigation, (3)communications, and (4)else. Normalizing (per $1000 \mathrm{sec)}$ the number of pilot's reactions to the workload lights, a candidate performance indicator, which may reflect the workload level as a function of aircraft configuration variations under SPIFR conditions were obtained. The last column in the table consists of similarly-normalized numbers of discrete throttle-plus-elevator trim adjustments (extracted from time-histories plots such as in Fig. $4 \cdot 3$ ).

Figure 4.3 contains also the continuous time-history of the longitudinal stick deflections $8 \mathrm{e}$. The RMS values of the yoke activity throughout a complete mission or along a particular flight segment may constitute performance indica-

1 Compilation of the detailed POR data, based on which these values were computed, and the pilot commentaries, is presented in Appendix E. 


\section{TABLE 4.1}

PORs, Reactions-to-Workload-Lights and Adjustments of Throttle-Plus-Elevator Trim as Candidate Performance Indicators

\begin{tabular}{|c|c|c|c|c|c|c|c|c|c|c|}
\hline \multirow{2}{*}{$\begin{array}{l}\text { CONFIG- } \\
\text { URATION }\end{array}$} & \multicolumn{2}{|c|}{$\begin{array}{l}\text { COMPLETE } \\
\text { TASK }\end{array}$} & \multicolumn{2}{|c|}{$\begin{array}{l}\text { HOLDING } \\
\text { PATTERN }\end{array}$} & \multicolumn{2}{|c|}{$\begin{array}{l}\text { GLIDE } \\
\text { SLOPE }\end{array}$} & \multicolumn{2}{|c|}{$\begin{array}{c}\text { AIRSPEED } \\
\text { RETRIMMING }\end{array}$} & \multirow{2}{*}{$\begin{array}{l}\text { REACTIONS } \\
\text { TO } \\
\text { WORKLOAD } \\
\text { LIGHTS }\end{array}$} & \multirow{2}{*}{$\begin{array}{l}\text { ADJUSTMENTS } \\
\text { OF THROTTLE } \\
\text { PLUS ELEVA- } \\
\text { TOR TRIM }\end{array}$} \\
\hline & CHR & SSR & CHR & SSR & CHR & SSR & CHR & SSR & & \\
\hline 1 & 3.0 & 3.25 & 3.0 & 3.25 & 3.0 & 3.25 & 3.25 & 3.25 & 28.2 & 49.5 \\
\hline 2 & 3.0 & 3.25 & 3.0 & 3.25 & 3.0 & 3.0 & 3.0 & 3.0 & 25.0 & 48.4 \\
\hline 3 & 3.75 & 3.75 & 3.5 & 3.5 & 3.75 & 3.5 & 4.0 & 4.0 & 26.9 & 54.9 \\
\hline 4 & 3.25 & 3.25 & 3.0 & 3.0 & 3.25 & 3.25 & 3.25 & 3.25 & 28.6 & 45.5 \\
\hline 5 & 3.0 & 3.0 & 3.0 & 3.0 & 3.0 & 3.0 & 3.5 & 3.5 & 25.0 & 46.0 \\
\hline 6 & 3.0 & 3.0 & 3.25 & 3.25 & 2.75 & 3.0 & 3.25 & 3.0 & 28.3 & 45.7 \\
\hline 7 & 3.0 & 3.0 & 3.0 & 3.0 & 3.0 & 3.0 & 3.25 & 3.25 & 28.5 & 42.8 \\
\hline 9 & 3.25 & 3.25 & 3.0 & 3.0 & 3.0 & 3.0 & 3.25 & 3.5 & 28.9 & 41.5 \\
\hline 10 & 3.5 & 4.25 & 3.0 & 3.0 & 3.25 & 4.5 & 4.5 & 4.5 & 29.2 & 57.4 \\
\hline 11 & 3.0 & 3.5 & 3.0 & 3.0 & 3.0 & 3.5 & 3.25 & 3.5 & 28.4 & 56.8 \\
\hline 12 & 3.25 & 3.5 & 3.0 & 3.0 & 3.0 & 3.25 & 4.25 & 4.5 & 29.2 & 41.3 \\
\hline 13 & 3.25 & 3.5 & 3.0 & 3.0 & 2.75 & 3.5 & 4.0 & 4.5 & 28.0 & 48.5 \\
\hline 14 & $3: 0$ & 3.5 & 3.0 & 3.25 & 3.0 & 3.0 & 3.5 & 4.0 & 28.6 & 51.6 \\
\hline 15 & 4.0 & 4.5 & 3.5 & 3.75 & 3.75 & 4.5 & 4.5 & 4.5 & 27.7 & 52.2 \\
\hline 16 & 3.0 & 3.0 & 3.0 & 3.5 & 2.75 & 3.0 & 2.75 & 2.5 & 28.8 & 39.0 \\
\hline
\end{tabular}




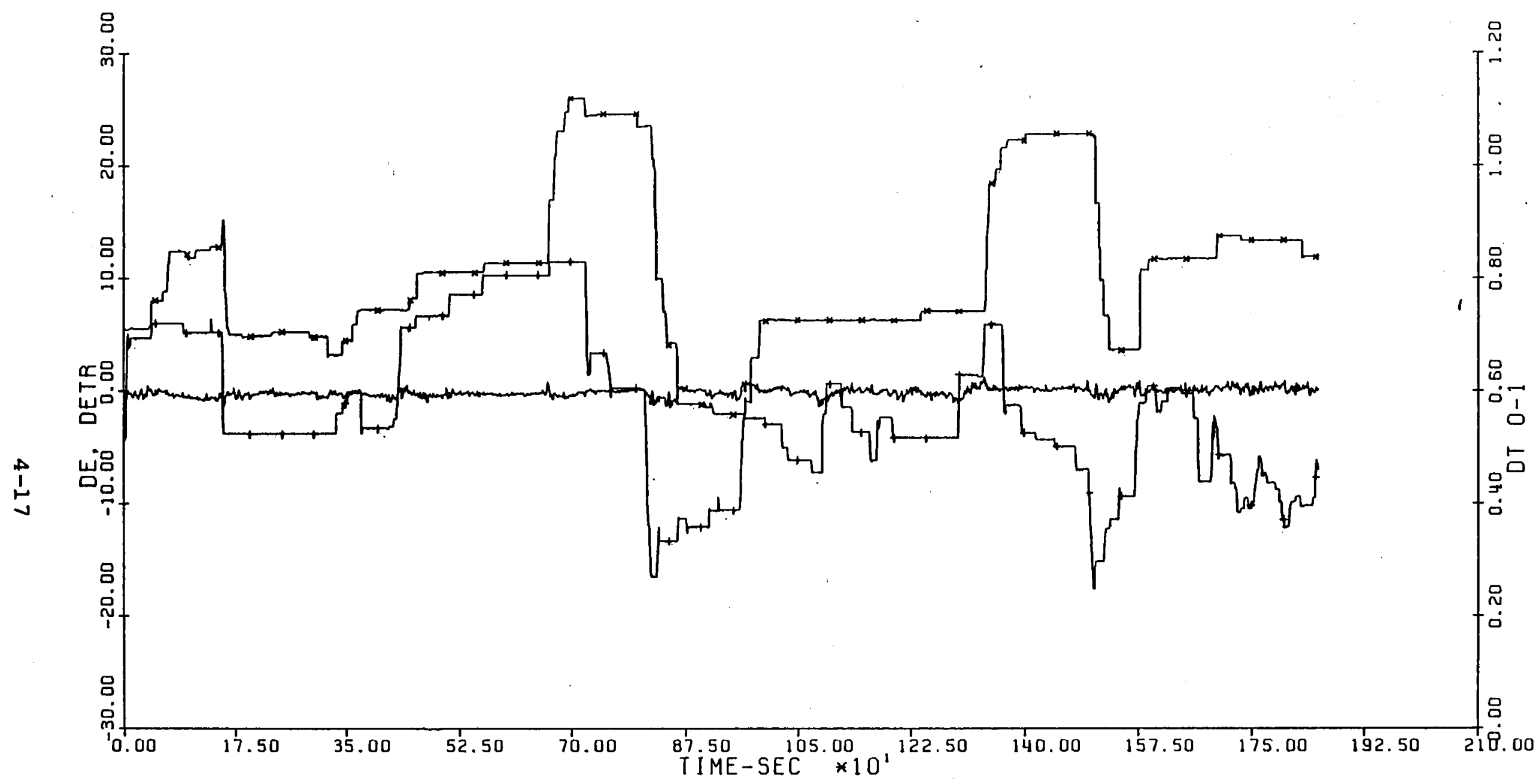

FLIGHT 1 DAY 7 MONTH 5 YEAR 1982

SYMBOLS ARE OI - , OEIA $X$

Figure 4.3: Time Histories of the Longitudinal Stick, the Throttle and the Longitudinal Trim Wheel 
tors. Note that cockpit controls manipulations (and tracking performances as elaborated later in this section), and not actual aerodynamic surfaces deflections were examined. The latter were a superposition of:the pilot's attempt to control the given configuration and of the implicit model following implementation commands, which simulated the particular configuration in flight. The RMS values of $\delta e(t)$ are,

$$
\mathrm{RMS}_{\delta_{e}}=\left[\left(\Sigma \delta e_{i}^{2}\right) /(n-1)\right]^{1 / 2}
$$

They are summarized in Table 4.2. In this section $\mathrm{n}$ is the number of points in the examined time series.

The determination of the start and the end points for the computation of the objective performance indicators required accurate identification of the different flight segments. This was done by concurrent examination of optimally smoothed flight path variables and controls plus VOR and glide slope tracking time histories, which were extracted from the data file magnetic tapes. Figures 3.9 to 3.12 and 4.3 to 4.5 constitute samples of this type of representation. In all figures, the symbols are $50 \mathrm{sec}$ apart. As obvious from Fig. 4.4, the actual ILs or MLS $^{2}$ tracking starts

2 The acronyms ILS and MLS stand for Instrument Landing system and Microwave Landing System, respectively. The MLS makes use of more advanced technology. For both systems the vertical needle tracking is referred to as 'localizer tracking', and horizontal needle tracking is referred to as 'glide slope tracking'. 
TABLE 4.2

Longitudinal Stick Deflections RMS Values as Candidate Performance Indicators

\begin{tabular}{|c|c|c|c|c|c|}
\hline $\begin{array}{l}\text { CONFIG- } \\
\text { URATION }\end{array}$ & $\begin{array}{c}\text { COMPIETE } \\
\text { MISSION } \\
\text { [DEG] }\end{array}$ & $\begin{array}{c}\text { CONSTANT } \\
\text { ALTITUDE } \\
\text { [DEG] }\end{array}$ & $\begin{array}{c}\text { CONSTANT } \\
\text { AIRSPEED } \\
\text { [DEG] }\end{array}$ & $\begin{array}{c}\text { CLIMB } \\
\text { [DEG] }\end{array}$ & $\begin{array}{c}\text { DESCENT } \\
\text { [DEG] }\end{array}$ \\
\hline - 1 & 0.5 & 0.4 & 0.4 & 0.5 & 0.5 \\
\hline 2 & 0.5 & 0.4 & 0.3 & 0.6 & 0.6 \\
\hline 3 & 0.5 & 0.5 & 0.3 & 0.4 & 0.5 \\
\hline 4 & 0.4 & 0.3 & 0.3 & 0.4 & 0.4 \\
\hline 5 & 0.5 & 0.4 & 0.4 & 0.5 & 0.5 \\
\hline 6 & 0.4 & 0.4 & 0.3 & 0.4 & 0.4 \\
\hline 7 & 0.4 & 0.3 & 0.3 & 0.5 & 0.4 \\
\hline 9 & 0.4 & 0.3 & 0.3 & 0.4 & 0.4 \\
\hline 10 & 0.3 & 0.3 & 0.3 & 0.4 & 0.4 \\
\hline 11 & 0.3 & 0.3 & 0.2 & 0.3 & 0.3 \\
\hline 12 & 0.3 & 0.3 & 0.3 & 0.4 & 0.4 \\
\hline 13 & 0.4 & 0.4 & 0.4 & 0.4 & 0.4 \\
\hline 14 & 0.4 & 0.4 & 0.2 & 0.4 & 0.3 \\
\hline 15 & 0.9 & 0.5 & 0.4 & 0.6 & 1.0 \\
\hline 16 & 0.3 & 0.3 & 0.3 & 0.4 & 0.3 \\
\hline
\end{tabular}


at about $t=1400$ sec. Figure 4.5 demonstrates the two VOR course deviation time histories; either or both may reach full scale deflection as the pilot selects cross-bearing radials, enters holding patterns, or crosses a VOR station. As for MLS tracking, correlation of the various time histories and the horizontal trajectory projections renders the intervals in which the pilot was supposed to track one of the two voR radials.

The tracking performance was quantified using two different methods One is the computation of the percentage of out-of-band dwelling time with respect to the complete MLS or VoR tracking time on a given segment. The permissible bands were set, in accordance with the pilot's strategy, to plus/minus one coarse division on the tracking instrument. This amounts to $\pm 2 \mathrm{deg}$ for VOR, $\pm 0.5 \mathrm{deg}$ for the localizer, and +0.2 deg on the glide slope. In Fig. 4.4 the band is shown for the localizer ("+" symbols for $50 \mathrm{sec}$ intervals). Note that it is shown with regard to the electrical zero reference, which has a -0.25 deg bias, as may be observed from from the instrument idling throughout the other segments of flight. The \pm 2 deg band for VoR tracking was superimposed on the plot of Fig. 4.5. The numerical values of the outof-band percentages are summarized in Table 4.3 .

The other method for quantifying the tracking performance was to compute the RMS values for deviation errors. For localizer tracking, 


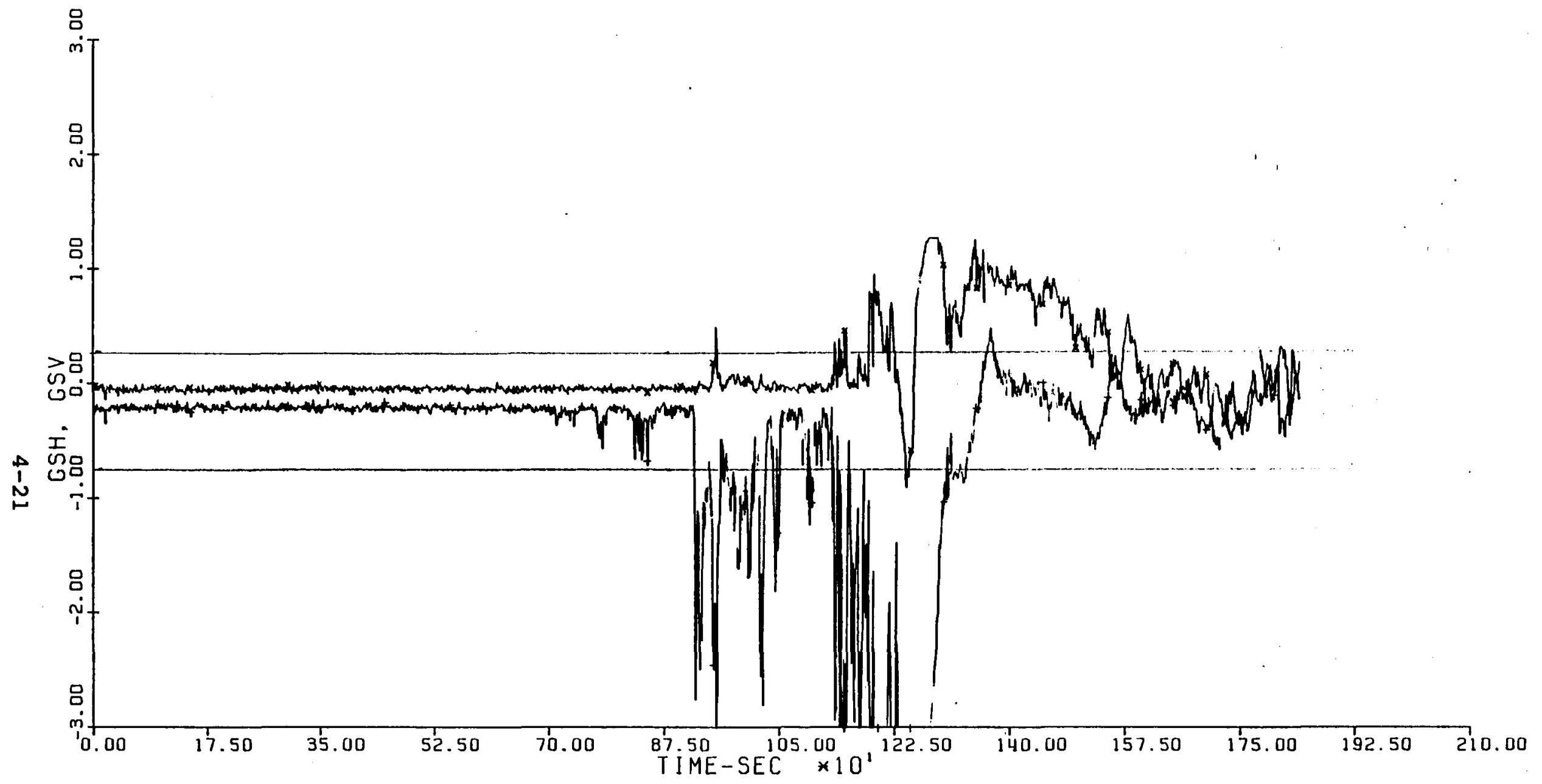

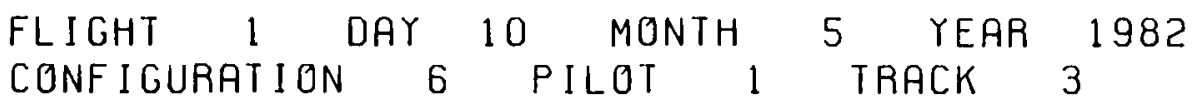

SYMBOLS ARE VORI/GSH *. VOR2/GSV $X$

Figure 4.4: Localizer and Glide Slope Deviations 


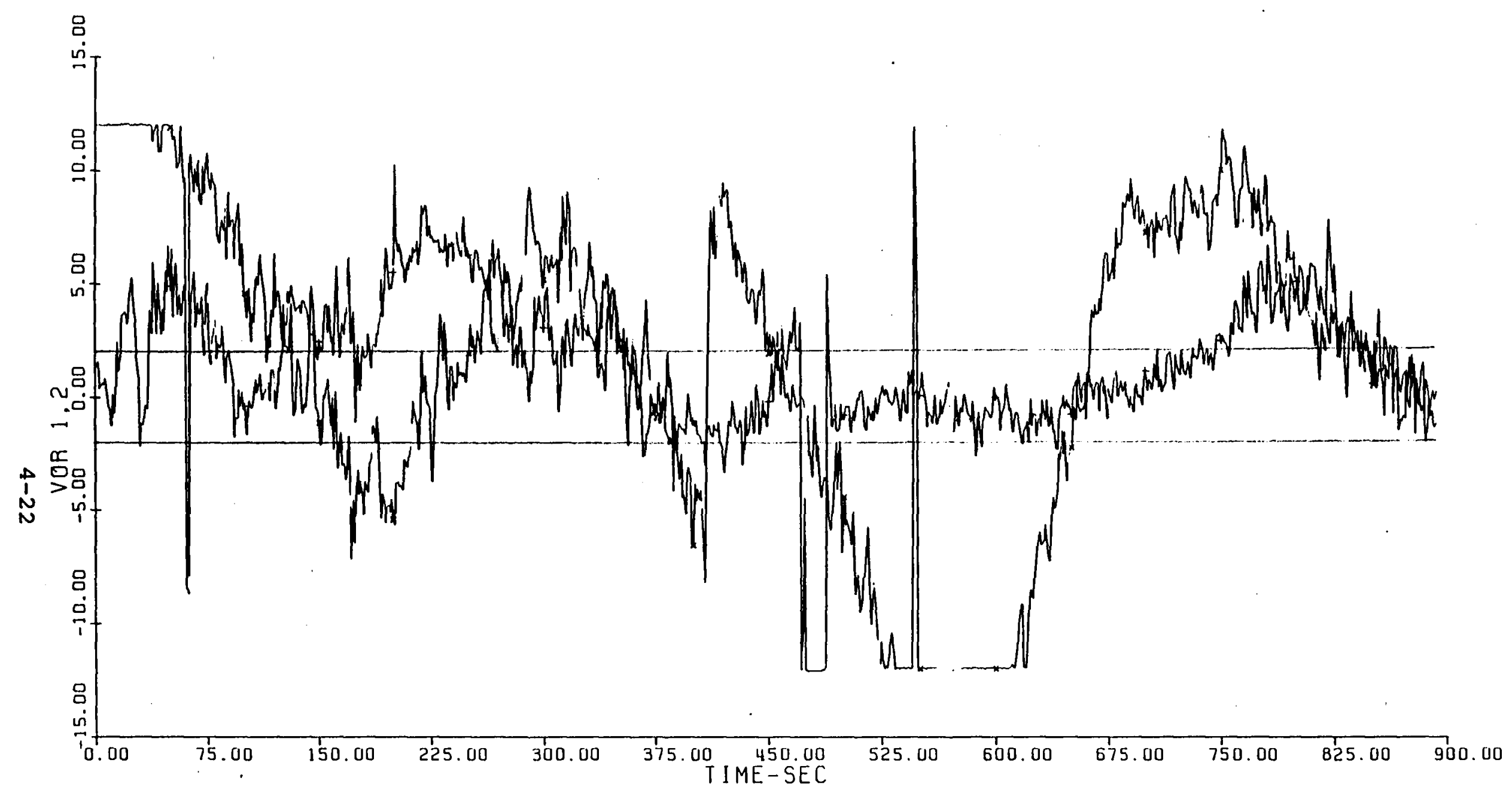

$\begin{array}{llllll}\text { FLIGHT } & 3 \text { MAY } & \text { MONTH } & 5 \text { YEAR } & 1982 \\ \text { CONFIGURATION } & 3 & \text { PILOT } & \text { TRACK } & 2\end{array}$

SYMEOLS aRE VORI/GSH *. VORZ/GSV $x$

Figure 4.5: VOR Deviations 


$$
\operatorname{RMS}_{1 O C}=\left[\left(\sum \epsilon_{L O C}^{2}\right) /(n-1)\right]^{1 / 2}
$$

for glide-slope tracking, -

$$
\mathrm{RMS}_{\mathrm{gs}}=\left[\left(\Sigma \epsilon_{\mathrm{gs}}^{2}\right) /(\mathrm{n}-1)\right]^{1 / 2}
$$

and for VoR-radial tracking,

$$
\mathrm{RMS}_{\mathrm{VOR}}=\left[\left(\Sigma \epsilon_{\mathrm{VOR}}^{2}\right) /(\mathrm{n}-1)\right]^{1 / 2}
$$

Obviously, the length of a segment, $n$, varies from segment to segment. All these evaluations of tracking performance are summarized in Table 4.3. It is important to realize that although they are not in the vertical plane, in which the configurations are alternated, the localizer and VOR "wandering" errors may reflect changes in the pilot's workload due to changes in the aerodynamic coefficients. Note that the RMS values and not the standard deviations of these "wandering" errors were computed to capture the integral effect of the SPIFR flight regime. Also, SPIFR tasks involve injection of events and discrete parameter variations (such as $\delta_{\text {etr }}$ or $\delta_{t}$ ), i.e., nonstationary conditions. Thus, the RMS values for tracking or holding errors may be the most appropriate performance indicators. 
TABLE 4.3

MLS and VOR Tracking RMS Values and Out-of-Band Time Percentages as candidate Performance Indicators

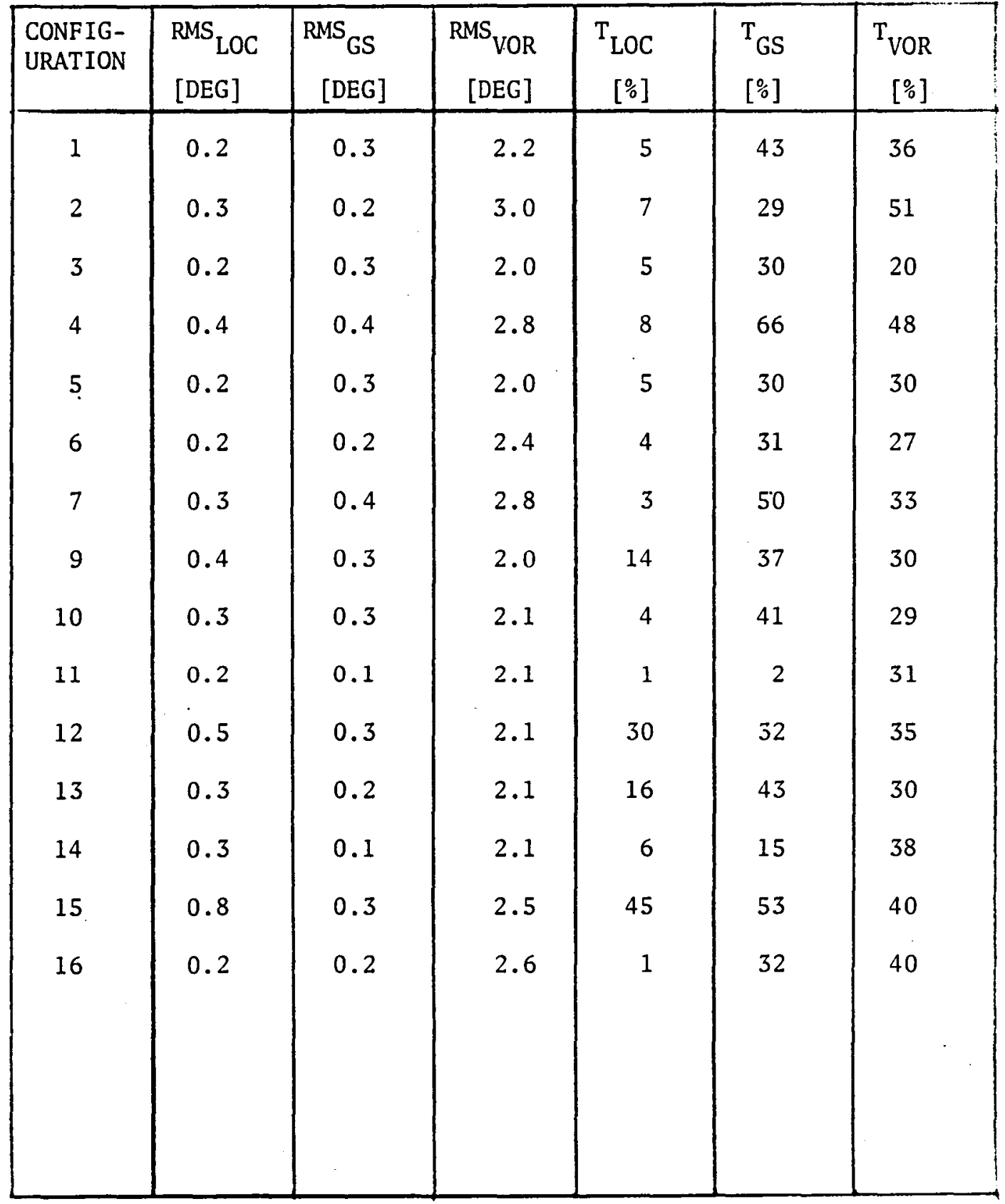


Based on information of the type contained in Fig. 3.12, additional performance indicators may be constructed. The evaluation pilot was instructed to fly controlled SPIFR missions, in which he had to track certain flight path variables, maintaining them at prescribed values. The values chosen represent typical practices for GA aircraft in a terminal air traffic control environment were:

* airspeed of 75 KIAS along holding patterns, climb and descents; and of 105 KIAS along straight and level segments .

* altitudes of $1500 \mathrm{ft}, 2000 \mathrm{ft}$ or $3000 \mathrm{ft}$ (depending on the particular segment of a specific flight track) for altitude holding tasks.

* rates of climb and descent of $500 \mathrm{ft} / \mathrm{min}$.

Instructing the pilot to track these values in appropriate flight segments was a secondary but realistic tracking workload, with prospects to bring out the effects of the aerodynamic configurations under SPIFR conditions. Note that Fig. 3.12 shows the indicated airspeed, which is the one usually tracked in the terminal area.

First, as for evaluation of the VOR and glide slope performance, the appropriate flight segments were identified; then the RMS values of deviations from holding altitude, airspeed and rate of climb or descent were computed. The 
out-of-band time approach was not pursued with regard to these variables because preliminary examination of the experimental results indicated that it may provide no decisive differentiation between the configurations flown.

Having identified the constant altitude segments, the following quantities were computed:

$$
\begin{aligned}
& \bar{h}=\left(\Sigma h_{i}\right) / n \\
& \overline{\Delta h}=\bar{h}-h_{c o m m} ; \quad\left(h_{c o m m}=1500,2000 \text { or } 3000 \mathrm{ft}\right) \\
& \mathrm{MS}_{\mathrm{h}}=\left(\Sigma \mathrm{h}_{i}^{2}\right) /(\mathrm{n}-1) \\
& \mathrm{RMS}_{\Delta \mathrm{h}}=\left[\Sigma\left(\mathrm{h}_{\mathrm{i}}-\overline{\mathrm{h}}\right)^{2} /(\mathrm{n}-1)\right]^{1 / 2}
\end{aligned}
$$

For constant airspeed segments,

$$
\begin{aligned}
& \overline{\mathrm{V}}=\left(\Sigma \mathrm{v}_{i}\right) / \mathrm{n} \\
& \overline{\Delta \bar{V}}=\overline{\mathrm{v}}-\mathrm{v}_{\mathrm{comm}} ; \quad\left(\mathrm{v}_{\mathrm{comm}}=75 \text { or } 105 \mathrm{KIAS}\right) \\
& \mathrm{MS}_{\mathrm{V}}=\left(\Sigma \mathrm{v}_{i}^{2}\right) /(\mathrm{n}-\mathrm{I}) \\
& \mathrm{RMS}_{\Delta \mathrm{V}}=\left[\Sigma\left(\mathrm{v}_{i}-\overline{\mathrm{v}}\right)^{2} /(\mathrm{n}-\mathrm{l})\right]^{1 / 2}
\end{aligned}
$$

For climb and descent segments, in addition to equations (4-25) to (4-28) with $\mathrm{V}_{\text {comm }}=75$ kIAS, the $\dot{\mathrm{h}}_{i}(t)$ time histories were computed and:

$$
\dot{\mathrm{h}}=\left(\Sigma \dot{\mathrm{h}}_{\mathrm{i}}\right) / \mathrm{n}
$$




$$
\operatorname{RMS}_{\Delta h}=\left[\left(\Sigma\left(\dot{h}_{i}-\dot{h}\right)^{2}\right) /(n-1)\right]^{1 / 2}
$$

The FORTRAN codes which took the information from the optimally-processed data file tapes and performed these computations, are given in Ref. 17. The results of their application are summarized in Tables 4.4 and 4.5 .

In the next section, the multiple regression algorithm is applied to the performance indicators prepared in this section versus the candidate SPIFR flying qualities criteria of Chapter 2. Before doing so, a closer examination of Tables 4.1 to 4.5 is called for. It may be noted that performance evaluations along holding pattern segments provide no distinct differentiation between the aerodynamic configurations flown, and they were not processed any further. A closer look at Table 4.2 reveals that all the numerical values of the stick deflection RMSs are lower than 1 deg, out of a stick range of over $30 \mathrm{deg} !$ This means that the yoke practically did not move from its zero position. It turned out, following pilot debriefing and reexamination of time histories of the type shown in Fig. 4.3, that an experienced pilot may work directly the longitudinal trim wheel, instead of first using the stick and then neutralizing the stick force. Thus, there was no sense in further processing of the data of Table 4.2. As a result of these observations, the number of performance indicators considered for the regression analysis was reduced to 22 . 
TABLE 4.4

Altitude and Altitude Gradient Tracking RMS Values as Candidate Performance Indicators

\begin{tabular}{|c|c|c|c|}
\hline \multirow{2}{*}{$\begin{array}{l}\text { CONFIGUR- } \\
\text { ATION }\end{array}$} & \multirow{2}{*}{$\begin{array}{l}\text { CONSTANT } \\
\text { ALTITUDE } \\
\text { TRACKING } \\
\text { RMS }_{h}[\mathrm{FT}]\end{array}$} & \multicolumn{2}{|c|}{$\begin{array}{l}\text { ALTITUDE GRADIENT } \\
\text { TRACKING, RMS }{ }_{h} \text { [FPS] }\end{array}$} \\
\hline & & CLIMB & DESCENT \\
\hline 1 & 25 & 0.2 & 1.0 \\
\hline 2 & 32 & 0.1 & 1.5 \\
\hline 3 & 29 & 1.3 & 1.0 \\
\hline 4 & 46 & 1.1 & 1.2 \\
\hline 5 & 45 & 1.0 & 1.0 \\
\hline 6 & 46 & 1.1 & 1.0 \\
\hline 7 & 42 & 1.0 & 1.1 \\
\hline 9 & 36 & 1.0 & 1.1 \\
\hline 10 & 60 & 0.2 & 1.0 \\
\hline 11 & 50 & 1.0 & 1.5 \\
\hline 12 & 30 & 0.5 & 0.1 \\
\hline 13 & 32 & 0.2 & 2.0 \\
\hline 14 & 24 & 1.0 & 0.5 \\
\hline 15 & 49 & 1.2 & 1.0 \\
\hline 16 & 27 & 0.5 & 0.5 \\
\hline
\end{tabular}


TABLE 4.5

Indicated Airspeed Tracking RMS Values as Candidate Performance Indicators

\begin{tabular}{|c|c|c|c|c|c|}
\hline \multirow{2}{*}{$\begin{array}{l}\text { CONFIGUR- } \\
\text { ATION }\end{array}$} & \multirow{2}{*}{$\begin{array}{l}\text { CONSTANT } \\
\text { AIRSPEED } \\
\text { SEGMENTS } \\
\text { RMS }_{\mathrm{V}}[\mathrm{FPS}]\end{array}$} & \multirow{2}{*}{$\begin{array}{l}\text { CLIMB } \\
\text { RMS }_{v}[\text { FPS }]\end{array}$} & \multicolumn{3}{|c|}{ DESCENT $\mathrm{RMS}_{\mathrm{V}}[\mathrm{FPS}]$} \\
\hline & & & $\begin{array}{l}\text { ON GLIDE } \\
\text { SLOPE }\end{array}$ & $\begin{array}{l}\text { EXCEPT FOR } \\
\text { GLIDE SLOPE }\end{array}$ & OVERALL \\
\hline 1 & 2.5 & 2.5 & 3.0 & 7.2 & 5.1 \\
\hline 2 & 2.5 & 1.5 & 4.0 & 8.0 & 6.3 \\
\hline 3 & 3.0 & 3.5 & 3.1 & 3.0 & 3.0 \\
\hline 4 & 3.0 & 4.5 & 4.0 & 3.0 & 3.5 \\
\hline 5 & 2.5 & 3.0 & 3.1 & 3.2 & 3.1 \\
\hline 6 & 2.0 & 2.5 & 4.0 & 4.5 & 4.3 \\
\hline 7 & 3.1 & 3.3 & 3.0 & 4.5 & 4.0 \\
\hline 9 & 4.0 & 3.5 & 3.5 & 3.5 & 3.5 \\
\hline 10 & 3.0 & 3.0 & 3.0 & 6.0 & 4.5 \\
\hline 11 & 3.0 & 3.5 & 4.0 & 8.1 & 6.3 \\
\hline 12 & 2.0 & 2.5 & 3.0 & 2.0 & 2.5 \\
\hline 13 & 3.5 & 4.0 & 3.0 & 4.5 & 4.0 \\
\hline 14 & 3.0 & 3.1 & 3.0 & 2.5 & 2.7 \\
\hline 15 & 3.5 & 5.0 & 6.0 & 3.0 & 4.2 \\
\hline 16 & 3.0 & 2.5 & 3.2 & 4.5 & 4.0 \\
\hline
\end{tabular}




\subsection{DERIVATION OF SPIFR FLYING QUALITIES CRITERIA}

This section reports on the application of multiple regression to the candidate theoretical criteria (vectors in Table 2.2) against each of the experimental performance indicators (section 4.2). First, as pointed out in section 4.1, a decision had to be made in each case whether a transformation of variables is required for any of the candidate criteria. Scatter plotting of the performance indicators versus each of the candidate criteria proved to be an efficient means for making such decisions. Examples of this approach are given in Fig. 4.6 and 4.7 . Figure 4.6 shows the distinct trend in the localizer tracking RMS error,

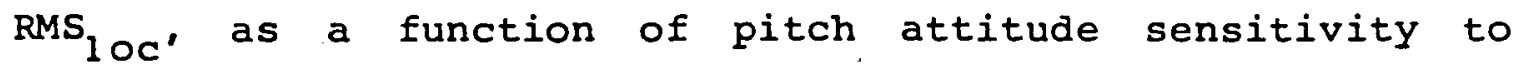
changes in airspeed, $\Delta \theta^{*} / \Delta v_{\text {comm }}$. Other candidate criteria did not exhibit any decisive effect on this performance indicator. Similarly, Fig. 4.7 suggests a relationship between the rate-of-descent holding RMS error, RMS ${ }_{h}$, and the phugoid total damping, $\left(\zeta \omega_{n}\right)_{p}$. It was observed from all scatter plots of this kind that there was no need for transformation of variables for the application of the regression algorithm.

The result of the multiple regression with regard to the RMS $_{10 c}$ performance ináicator is, 


$$
\mathrm{RMS}_{\text {lOC }}=-0.19-1.0\left(\Delta \theta^{*}[\mathrm{deg}] / \Delta \mathrm{V}_{\mathrm{comm}}[\mathrm{kt}]\right)
$$

with an $\alpha=0.05$ statistical significance level and zero values for all other regression coefficients. The correlation coefficient $R^{2}$ equals 0.74 , i.e. 74 , percent of the experimental results are explained by the relationship in eq. (4-31). With regard to hypothesis testing, $F_{\text {observed }}=36.8$ and as the statistical degrees of freedom are $n=15$ and

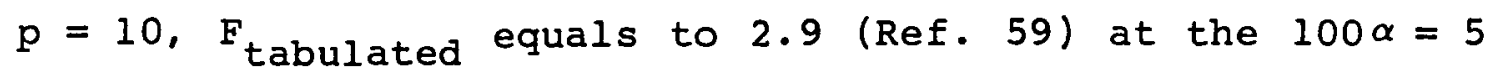
percent level. Fobserved $\gg F_{\text {tabulated, i.e., the result is }}$ significant at the level $\alpha=0.05$, and the null hypothesis is, indeed, rejected. The same criterion is also singled out by several other independent performance indicators, such as $\mathrm{T}_{10 \mathrm{c}}$ and $\mathrm{RMS}_{\mathrm{Vgs}}$.

To derive the numerical value for the $\Delta \theta^{*} / \Delta \mathrm{V}_{\text {comm }}$ flying qualities criterion, recall from the previous section that the pilot tries to keep the localizer needle within $0.5 \mathrm{deg}$ of the center. Substitution of this value into eq. (4-31) yields,

$$
\Delta \theta^{*} / \Delta \mathrm{v}_{\mathrm{comm}} \geqslant-0.7 \mathrm{deg} / \mathrm{kt}
$$

Similar considerations applied to the regressed relationships, $\mathrm{T}_{10 \mathrm{C}}\left(\Delta \theta^{\star} / \Delta \mathrm{V}_{\text {comm }}\right)$ and $\mathrm{RMS}_{\mathrm{Vgs}}\left(\Delta \theta^{*} / \Delta \mathrm{V}_{\text {comm }}\right)$, crosscheck this result. A design which features an $\mathrm{L}_{a}$ aerodynamic derivative that is too low, may be problematic in complying with this criterion. 
Regression for the $\mathrm{CHR}_{\text {whole' }} \mathrm{SSR}_{\text {whole' }} \mathrm{CHR}_{\mathrm{gS}}$, and $\mathrm{SSR}_{\mathrm{gS}}$ performance indicators identified another SPIFR flying qualities criterion, which also may be related to variations in $L_{a}$. This criterion is the stick force sensitivity to changes in airspeed, $\Delta \mathrm{f}_{\mathrm{s}}^{*} / \Delta \mathrm{v}_{\mathrm{comm}}$. For example, for $\mathrm{CHR}_{\text {whole' }}$ the result is,

$$
\mathrm{CHR}_{\text {whole }}=2.5+1.4\left(\Delta \mathrm{f}_{\mathrm{s}}^{*}[\mathrm{lb}] / \Delta \mathrm{V}_{\mathrm{comm}}[\mathrm{kt}]\right)
$$

Although CHR $=6.5$ is still considered flyable, note that the philosophy of this research was to isolate the airframe dynamic response from other factors, such as turbulence. Thus the criterion ought to be computed for a lower bound of this performance indicator (this reasoning also was applied in Ref. 10). Substitution of $\mathrm{CHR}=4.5$ into eq. $(4-33)$ yields,

$$
\Delta \mathrm{f}_{\mathrm{s}}^{*} / \Delta \mathrm{v}_{\mathrm{comm}} \mathrm{l}_{\max }=1.4 \mathrm{lb} / \mathrm{kt}
$$

As mentioned at the beginning of this section (Fig. 4.7), the phugoid total damping criterion, $\left(\zeta \omega_{n}\right)_{p}$ was deduced from the RMS error in rate-of descent holding, RMS ${ }_{h}$ '

$$
\mathrm{RMS}_{\dot{\mathrm{h}}}=1.96-17.81\left(\zeta \omega_{n}\right)_{\mathrm{p}}
$$

The permissible minimum value of $\left(\zeta \omega_{n}\right)_{p}$ ' allowing $\mathrm{RMS}_{h}$ up to $100 \mathrm{fpm}$, should be,

$$
\left(\zeta \omega_{n}\right)_{\min } \cong 0.02
$$


Typically for light aircraft, $\omega_{\mathrm{np}}$ is about $0.34 \mathrm{rad} / \mathrm{sec}$ (Table B.1). Thus, the phugoid damping ratio itself may be required to stay in the range,

$$
\zeta_{p} \geqslant 0.06
$$

A configuration with $\mathrm{TD}_{\mathrm{V}}$ aerodynamic coefficient, that is too low, may be problematic in this respect. Note that the actual experimental result is reflected in eq. (4-36), and $\zeta_{p}$ was computed for comparison with Ref. 9. The latter requires,

$\zeta_{p}>0.04$ for normal operations (Level 1)

$\zeta_{p}>0$ for Level 2 (definition of Level 3

$$
\text { excludes SPIFR operations) }
$$

Comparing eq. (4-38) and (4-37), it is suggested that at least the numerical value for Level 1 in eq. $(4-38)$ be adopted for the FAR 23 (Ref. 5).

Relating SPIFR experimentally-derived flying qualities criteria to existing military or civil specifications, as exercised above for $\zeta p^{\prime}$ may provide an important perspective. For example, variation in $\dot{h}$, which brought out the phugoid damping ratio as a SPIFR criterion, may also affect the pilot's performance in an additional way. Flying the 


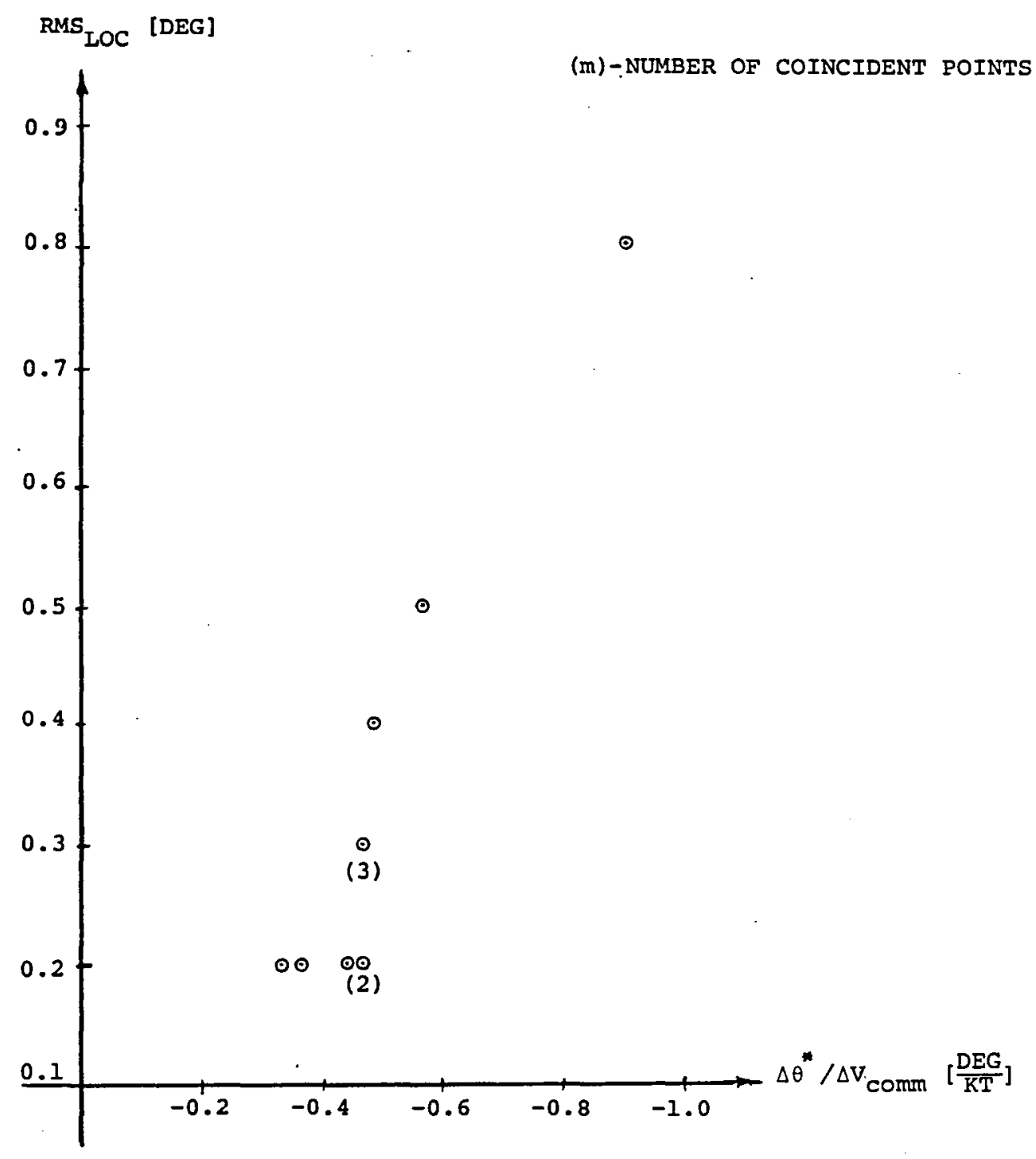

Figure 4.6: Localizer Tracking RMS Error as a Function of Pitch Attitude Sensitivity to Changes in Airspeed.

$\stackrel{m}{\sim}$ 


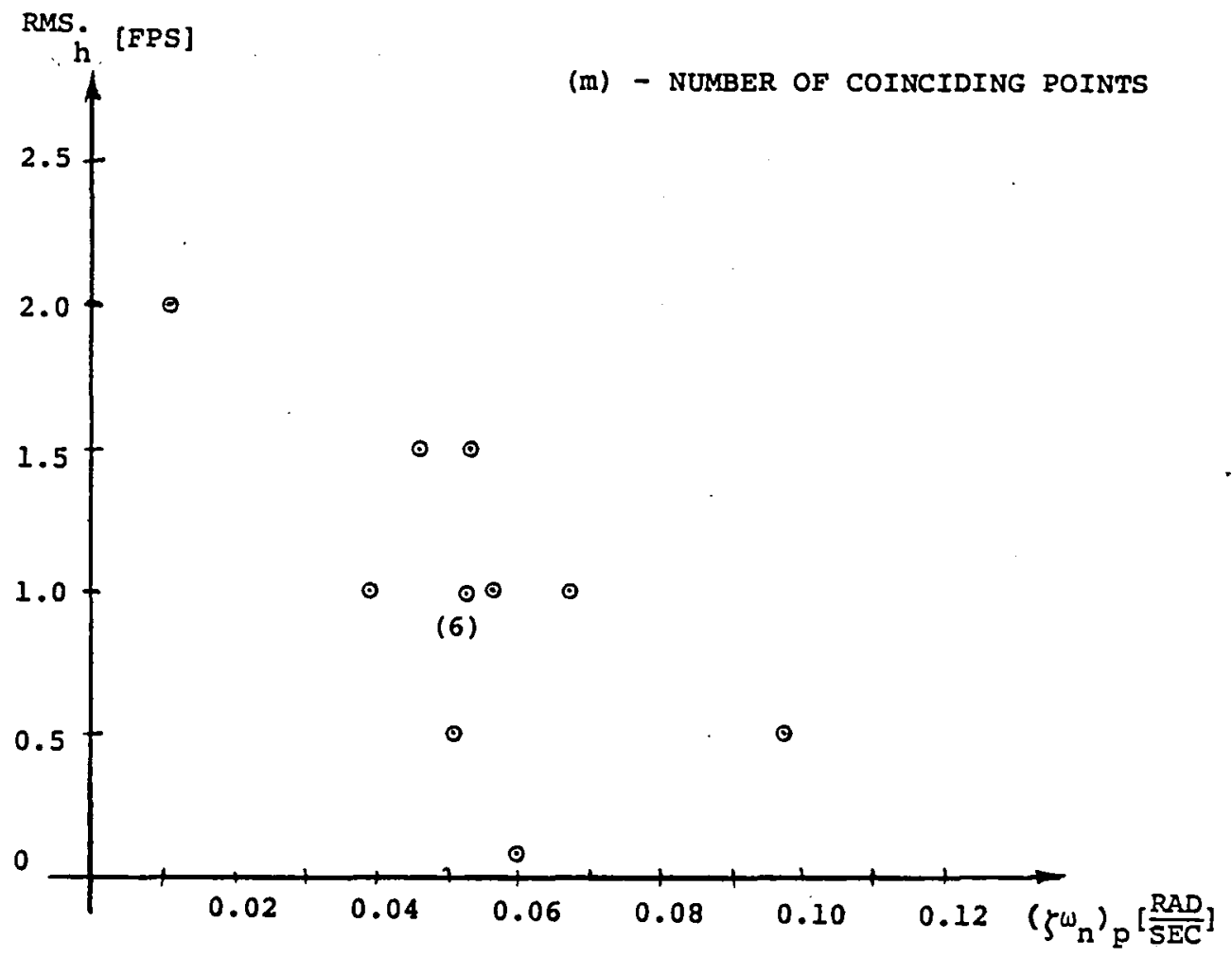

Figure 4.7: Rate-of-Descent Holding RMS Error Versus the Phugoid Total damping. 
landing approach, the pilot should be able to maintain airspeed using only the elevator; throttle adjustments in this critical flight phase may increase his workload to an unsafe level. To prevent this, Ref. 9 sets detailed requirements for flight path stability, which "is defined in terms of flight path angle change where the airspeed is changed by the use of pitch control only". As the flight path angle sensitivity to change in airspeed, $\Delta r / \Delta v$ is,

$$
\Delta \gamma / \Delta \mathrm{v}=(1 / \mathrm{v}) \Delta \dot{\mathrm{h}} / \Delta \mathrm{v}
$$

it is obvious that $\dot{h}$ may affect the pilot's performance also through its airspeed change gradient.

As shown in Ref. 70, flight path stability variations correspond to the power required curve for a given configuration. In terms of aerodynamic coefficients,

$$
\begin{aligned}
\Delta \gamma / \Delta V \cong & -(1 / g)\left[D_{v}-D_{a}\left(L_{v} / V\right) /\left(L_{a} / V\right)\right] \cong \\
& (1 / g)\left[x_{u}+\left(g-x_{w} V\right)\left(-z_{u} / V\right)\left(-z_{w}\right)\right]
\end{aligned}
$$

Substitution of the numerical values from Chapter 2 yields $\Delta \gamma / \Delta \mathrm{V}$ for all SPIFR configurations. The results of this computation are summarized in Table $4.6 .^{3}$

3 Configurations 15 and 16 did not affect flight path stability, because the ratio $D_{a} / L_{a}$ was constant for their in-flight simulation. 
TABLE 4.6

Computation of Flight Path Stability Parameter for SPIFR Configurations

\begin{tabular}{|c|c|}
\hline CONFIGURATION & $\begin{array}{c}\Delta \gamma / \Delta V \\
{[\mathrm{DEG} / \mathrm{KT}]}\end{array}$ \\
\hline to $10,15,16$ & -0.045 \\
11 & -0.105 \\
12 & +0.015 \\
13 & +0.204 \\
14 & -0.294 \\
\hline
\end{tabular}

The flight path stability requirement in Ref. 9 is: "the curve of flight path angle versus true airspeed (Fig. 4.8) shall have a local slope which is negative or less positive than:
a. Level 1 ---- $0.06 \mathrm{deg} / \mathrm{kt}$
b. Level 2 - - $0.15 \mathrm{deg} / \mathrm{kt}$
c. Level 3 - $0.24 \mathrm{deg} / \mathrm{kt}$ 
Comparing the values in Table 4.6 to these requirements, it may be anticipated that only configuration No. $13\left(\mathrm{x}_{\mathrm{u}}=0\right)$ would be of serious concern. Although this is insufficient for statistical analysis, it should be kept in mind that the pilot may be sensitive to $\Delta y / \Delta V$ as well as to $\zeta_{\mathrm{p}}$.

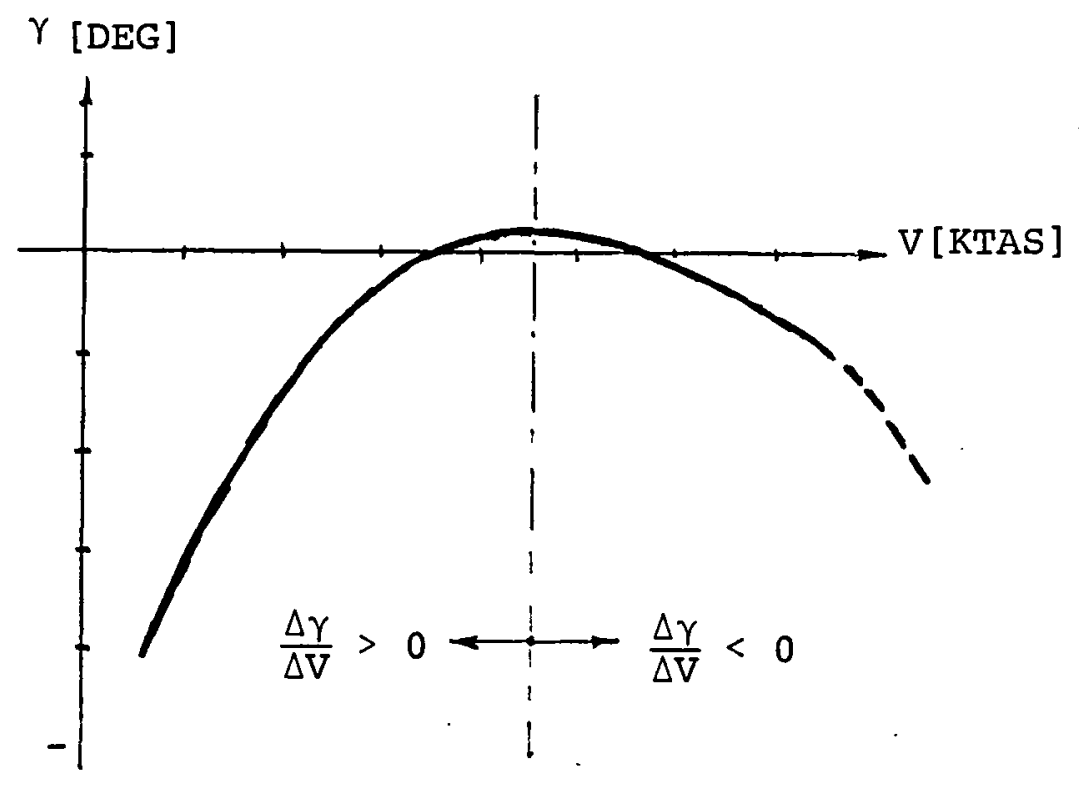

Figure 4.8: Flight Path Angle Versus Airspeed on Descent

Additional insight may be gained by evaluating the pitch attitude sensitivity criterion (eq. (4-32)) in view of Fig. 4.8. As obvious from Table B.2, the throttle adjustments, 
which are required for steady-state airspeed retrimming, are relatively small. Thus, although the two problems are not identical, the $\Delta \theta^{*} / \Delta \mathrm{v}_{\text {comm }}$ criterion suggests that a too strong negative slope in Fig. 4.8 (the right side of the curve) may also be disturbing to the pilot of a fast-descending airplane.

With regard to the stick force sensitivity, Ref. 5 addresses this issue qualitatively: "The stick force must vary with speed so that any substantial speed change results in a stick force clearly perceptible to the pilot". Any possible quantification of this stick force sensitivity criterion, $\Delta \mathrm{f}_{\mathrm{s}}^{*} / \Delta \mathrm{V}_{\text {comm' }}$ is important because it is equivalent to a perception by the pilot of having direct control of velocity. This perception simplifies the piloting task by reducing the number of integrations required for proper control. Such a quantitative upper bound is indicated by the results of this research (eq.(4-34)). This correlation between the derived SPIFR criteria and the requirements in the civil and military specifications enhance the findings of this research. Thus, the flying qualities criteria in eq. (4-32), (4-34) and (4-38) constitute guidelines, which the GA aircraft designer should consider.

Referring back to the fifteen aerodynamic configurations which were examined in this research, only $L_{\alpha}$ and $x_{u}$ were 
found to feature ranges of values, which may be very significant under SPIFR conditions (their physical significance was discussed in Section 2.2). All other derivatives did not stand out above the high background navigation/communication workload. Also, note that each of the performance indicators, which did confirm candidate criteria as actual SPIFR flying qualities criteria, rendered a single dominant criterion (with an $\alpha=0.05$ statistical significance level). All other performance indicators failed to exhibit trends as a function of the aerodynamic configurations. This includes both the physically obvious metrics, such as RMS values of airspeed and altitude holding errors, and the artificiallydeviced indicators, such as the normalized frequency of reaction to workload lights.

An important by-product of the above analysis is the comparative evaluation of the CHR and the SSR pilot opinion rating scales. In representations of the type of Fig. 4.6 the SSR workload ratings are practically the same as the CHR, and provide the same trends of variation. In few cases they differ by up to 0.5 rating. As this is less than the generally accepted $\sigma_{\mathrm{CHR}^{\prime}}$ which is 1.0 rating, the use of SSR along with CHR in SPIFR experiments appears to be redundant. Although this observation relies on ratings by a single pilot, this author believes that it holds in the range 2.0 to 5.0, which was obtained in the SPIFR flight tests. Also, as 
shown in the above analysis, objective performance metrics are important complements to subjective PORs.

The FORTRAN and JCL codes for computation of the performance indicators, the scatter print plotting and the statistical analysis are given in Ref. 17. 


\section{Chapter 5}

\section{SUMMARY, CONCLUSIONS AND RECOMMENDATIONS}

\subsection{SUMMARY OF THE RESEARCH METHODOLOGY}

This research addressed the issue of degradation in GA safety which occurs under SPIFR conditions. The "man-machine" performance in this flight regime is a function of several factors including aircraft dynamic response and increased navigation/communication workload. As a first step toward alleviating the SPIFR safety problem, the relative importance of these two major factors was determined. To achieve this objective a self-contained methodology that involves theoretical and experimental aspects was developed. The airframe response in the low-frequency range was varied in flight testing, and pilot's performance and workload metrics were obtained as a function of these variations.

The experiment-based performance indicators included both pilot judgment and objective ability to carry out typical SPIFR tasks such as MLS and VOR radial tracking and altitude and airspeed holding. The range of variation for each of the aerodynamic coefficients was chosen to cover both the 
existing light aircraft fleet and recent trends in GA design. For each of the chosen configurations, frequency- and time-domain-related candidate flying qualities criteria were obtained from theoretical considerations. Finally, the multivariate regression algorithm was applied to this data base, which consisted of a set of vectors of the candidate flying qualities criteria (horizontal axes for the regression) and of the performance indicators' vectors (vertical axis for the regression analysis).

For accurate computation of the performance indicators, an efficient optimal flight path reconstruction algorithm was developed. Its mathematical modeling accounted for Earth rotation and geometry. Using as inputs aerodynamic and inertial measurements and outputs of a microprocessorbased multiple DME scanner, it provided results in the True North-East navigational coordinates. As the model was nonlinear, the extended Kalman filter algorithm was used. To achieve better filter performance in terms of accuracy and robustness, the mathematical model was partitioned into lower-order submodels, which were processed sequentially. Accurate tuning was obtained by maintaining a proper balance between state covariances and the covariances of the measurement residuals. The Rauch-Tung-Striebel optimal smoother algorithm and Fraser's smoothability condition were employed to enhance accuracy and efficiency in trajectory estimation. 
The accuracy rendered by this optimal flight path reconstruction scheme was demonstrated on actual flight test data. It was shown to provide an assessment of the flight technical error of the VOR/VOR navigational mode, as well as the SPIFR performance indicators.

\subsection{CONCLUSIONS AND RECOMMENDATIONS}

The following important results were derived:

* A phugoid mode that is too lightly damped, produces unsatisfactory flying qualities, and it may have an adverse effect on safety in the SPIFR flight regime. It is suggested that the phugoid damping ratio should not be lower than 0.04, which is consistent with the criterion of the military flying qualities specifications.

* The current FAA reference to stick force sensitivity, $\Delta \mathrm{f}_{\mathrm{s}}^{*} / \Delta \mathrm{v}_{\mathrm{comm}}$ ' is qualitative. Any quantification of this criterion is important, because it is equivalent to a perception by the pilot of having direct control of velocity, which simplifies the piloting task. Such a quantitative upper bound is indicated by the results of this research: $\Delta f_{s}^{*} / \Delta v_{\text {comm }} \leqslant 1.41 \mathrm{~b} / \mathrm{kt}$.

* The pitch attitude sensitivity to airspeed change from one trimmed flight condition to another, $\Delta \theta^{*} / \Delta \mathrm{V}_{\mathrm{comm}}$ is suggested as a new criterion, for the SPIFR flying 
qualities. Its value should not be lower than $-0.7 \mathrm{deg} / \mathrm{kt}$.

Experiment-based objective performance metrics, such as tracking error RMS values, were found to agree with a single subject's opinion in SPIFR task evaluation. Also, the current research suggests that the Cooper-Harper pilot opinion scale is a good indicator of perceived pilot workload. Its results indicate that the SPIFR navigation/communication workload is important, compared to workload due to low-frequency aircraft dynamics.

\section{Recommendations:}

* To improve the safety of General Aviation single-pilot IFR operations, it is suggested that flying qualities criteria for the SPIFR flight regime, which were derived in the course of this research, be considered for incorporation into the Federal Aviation Regulations (FAR) 23 .

* The flight path reconstruction methodology, developed here, can provide flight technical error evaluation of other navigational systems, such as LORAN-C. It may be used in such projects as the NASA Langley $\mathrm{TCV}^{1}$ and to investigate, statistically, flight path deviations by

1 Terminal Configured Vehicle project, which uses the Boeing 737 as a flight-test bed. 
recording DME ranges along with inertial data, altitude, and airspeed.

* This systematic approach, which combines theory and flight testing, should be employed to identify lateraldirectional flying qualities criteria for SPIFR operations. 


\section{Appendix A \\ AIRCRAFT AND DATA ACQUISITION SYSTEM PREPARATION}

This Appendix describes the preparation of the in-flight simulator and of the onboard digital data acquisition system for SPIFR flight testing. Extensive engineering and technical effort was required for aircraft modifications and rewiring, for new avionics system installation, and for onboard experimental setup integration. The results of this effort are summarized in the following sections.

\section{A.1 AIRCRAFT SYSTEM MODIFICATIONS}

The Avionics Research Aircraft (ARA) is a Ryan Navion (N5113K) that has been modified into a fly-by-wire (FBW), variable-stability aircraft (Fig. A.l). It is capable of simulating a variety of other aircraft using feedback control and command augmentation. The ARA is equipped to measure attitude, angular rates, and linear accelerations in three axes, aerodynamic angles $(\alpha, \beta)$, airspeed, altitude, and a number of other flight variables. Details of the ARA FBW system can be found in Ref. 71 . 
The evaluation pilot was to fly a SPIFR mission with the ARA responding as a desired configuration. In an emergency, the safety pilot could override the FBW system and take direct control of the aircraft (Fig. A.2).

To be used with the SPIFR program, the ARA had to undergo extensive modifications. These included:

* Design and installation of a modular instrument panel.

* Acquisition and installation of a modern navigation/ communication instrument package.

* Addition of secondary workload devices in the cockpit.

Figure A.3 illustrates the ARA's modular display panel configuration, with the evaluation pilot's station on the left, the safety pilot's station on the right, and the Bendix BX-2000 navigation/communication stack separating the two. The Distance Measuring Equipment (DME) readout is mounted on a switching panel at the top of the radio stack. The Very-high-frequency-Omni-Range (VOR) navigation/communication unit is located under the switching panel. The blank space below this unit is reserved for an Automatic Direction Finder (ADF) and a transponder.

The DME unit has been integrated into the experimental electronics, maintaining the capability to sequence the available navigational stations automatically (through microprocessor control). The importance of this option is 


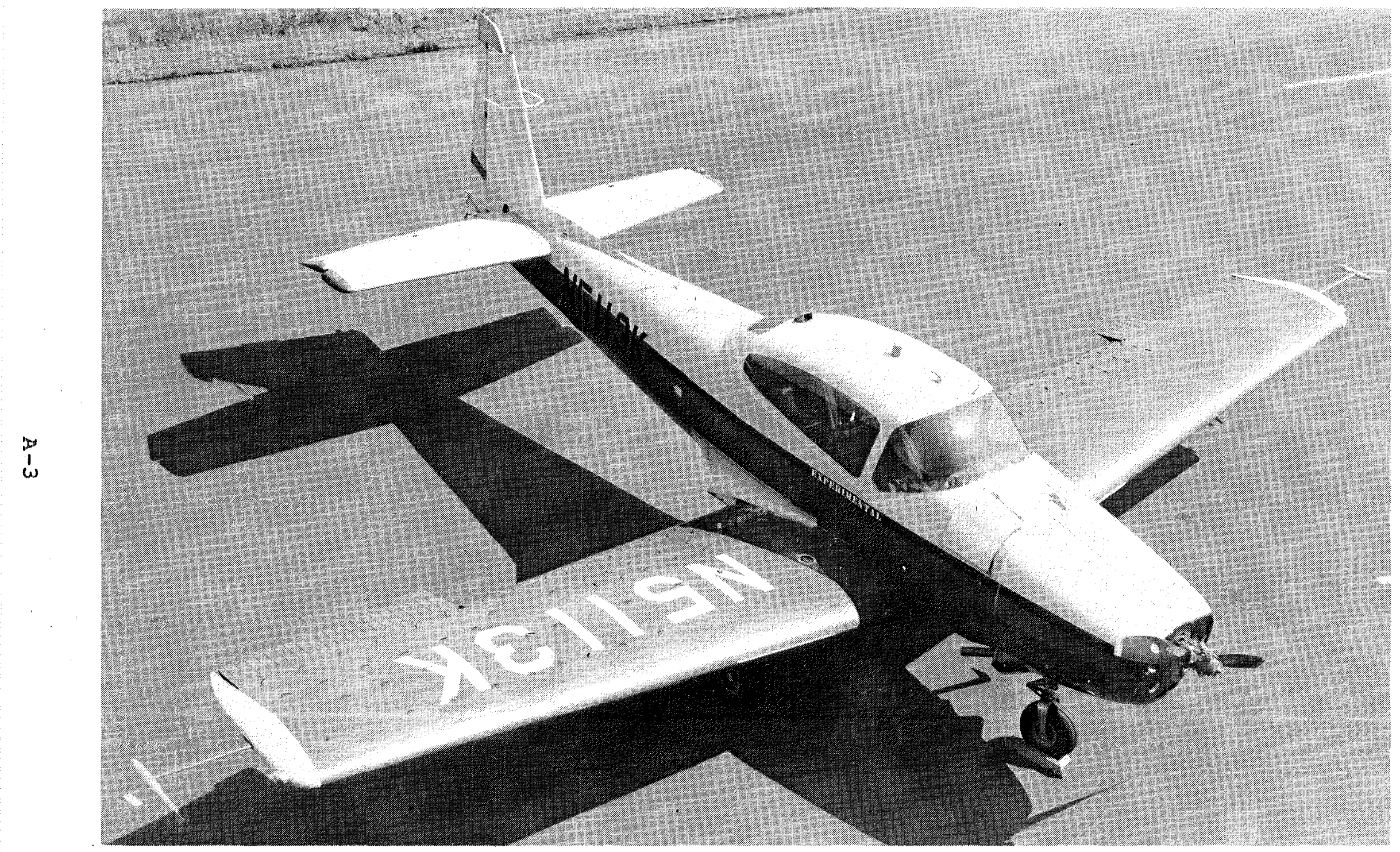

Figure A.1: Avionics Research Aircraft, Navion N5113K 


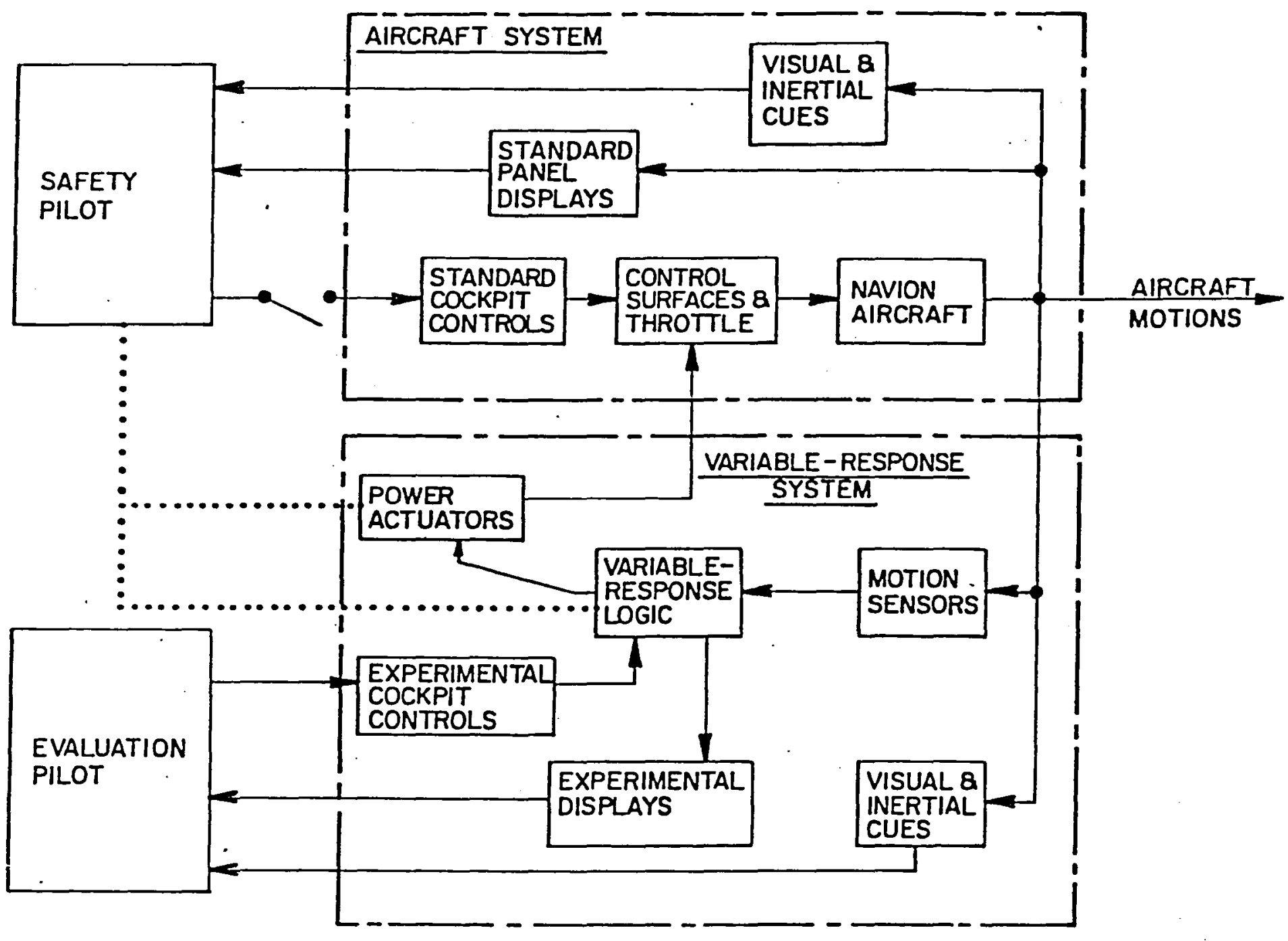

Figure A.2: Overview of the ARA In-Flight Simulator system 
discussed in Section 2.5. The technical implementation details may be found in Ref. 72 .

The safety pilot's panel is a permanent fixture, with conventional instruments and elements for control of the variable-stability system. The latter occupy the right side of the panel and the lower and middle consoles. The evaluation pilot's panel can be removed as a unit to facilitate installation of alternate panels for other investigations. Secondary workload meters, lights, and switches also have been added to the panel.

The secondary workload meters are additional instruments slaved to the onboard microprocessor, which occasionally forces the needles into their "red zones". Alternately, the pilot can be asked to extinguish lights turned on (pseudorandomly) by the microprocessor program. It is also possible to simulate typical communication workload by blending audio inputs from a pre-recorded tape with various instructions radioed from the ground on the flight test frequency. Simulated air traffic control vectoring is provided on the same channel, with instructions given by the safety pilot. 


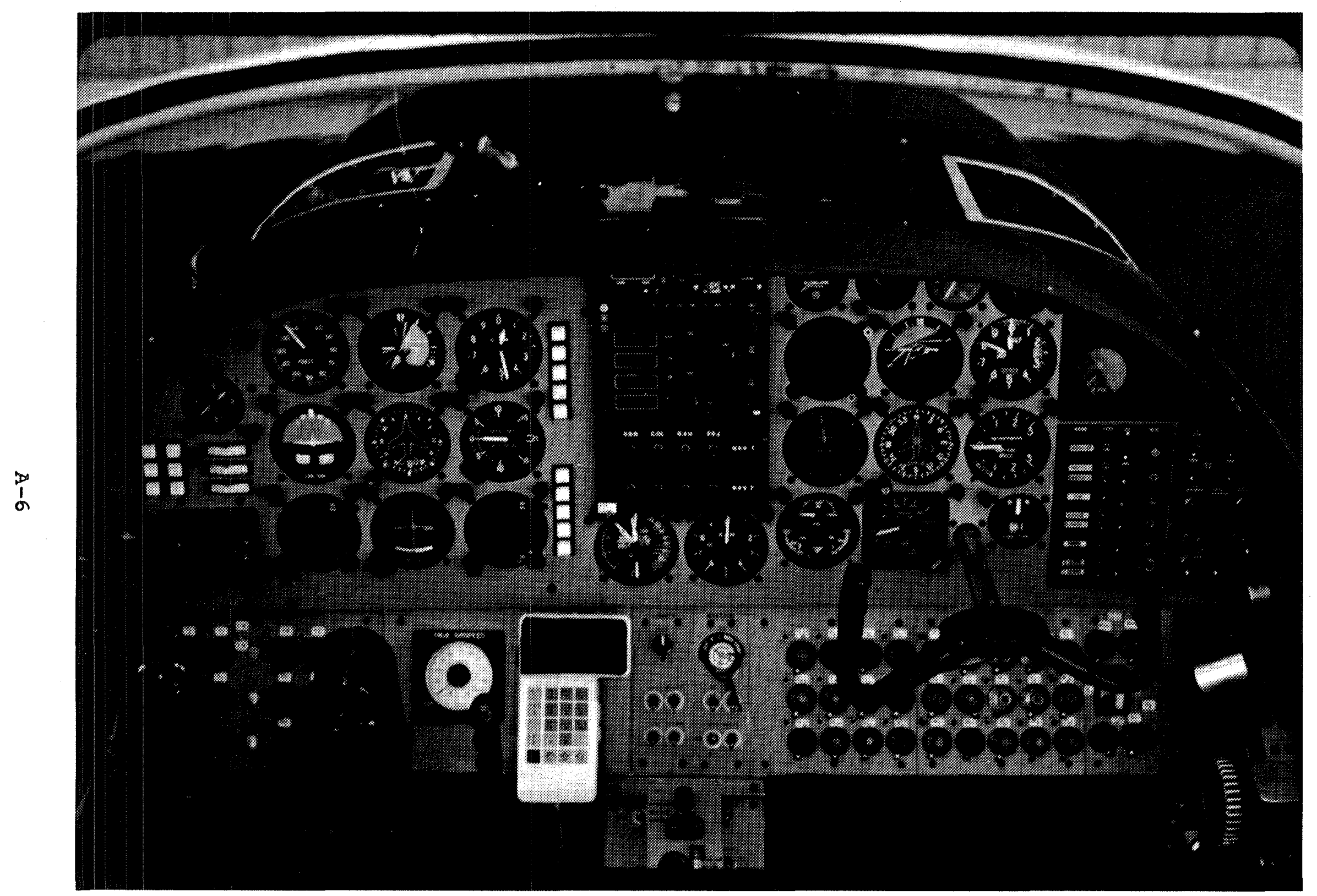

Figure A.3: Cockpit Displays of the Avionics Research Aircraft. Modular SPIFR Evaluation Pilot Panel at Left. 


\section{A.2 INSTRUMENTATION AND DATA RECORDING SYSTEM}

The SPIFR digital data acquisition system is illustrated in Fig. A.4. It is built around the SPIFR microcomputer, which uses the z-80A central processing unit and the Am9511 mathematics processor in a Multibus ${ }^{T M}$ architecture. As currently configured, the SPIFR microcomputer contains $48 \mathrm{~K}$ bytes of RAM (Random Access Memory) and 16K bytes of PROM (Programmable Read-Only Memory). It accepts 32 analog inputs and produces 6 analog outputs.

The ARA's safety pilot communicates with the SPIFR microcomputer through a hand-held Control/Display Unit (CDU), the Termiflex HT/4. The pilot is able to start and stop processing or recording through the CDU, change stored numerical values, and so on. Conversly, the CDU can display internally triggered error messages to the safety pilot. The evaluation pilot normally is unaware of the SPIFR Microcomputer's operation, other than through secondary workload stimuli and responses.

Analog and digital inputs and outputs shown in Fig. A.4 are, for the most part, self-explanatory. Tables A.l and A. 2 contain lists of inputs and outputs. The SPIFR Microcomputer obtains its analog inputs from the Digital Avionics Research System (DARE) Junction Box (J-Box) previously in- 


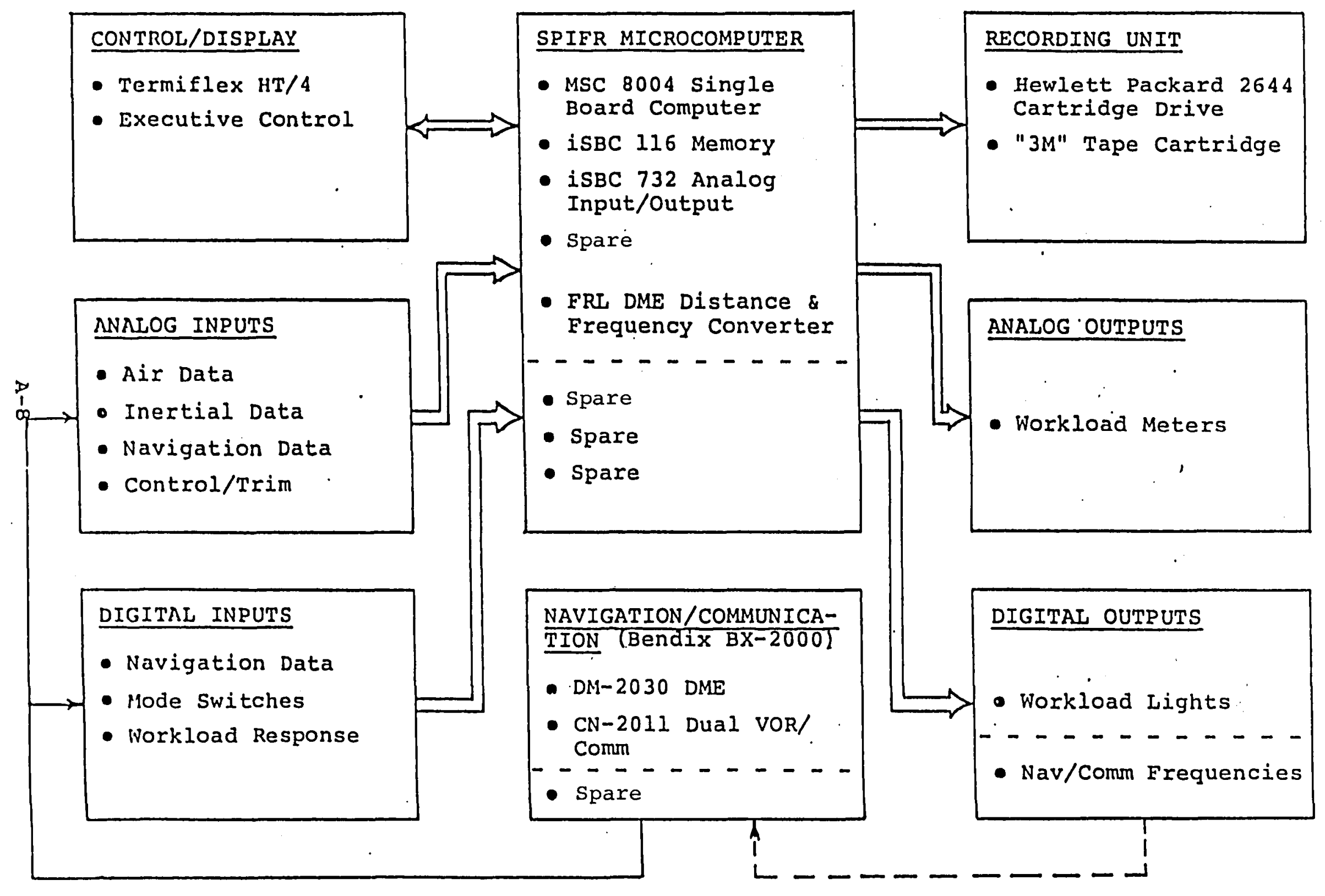

Figure A.4: SPIFR Digital Data Recording System. 
TABLE A. 1

Input Assignments for SPIFR Digital Data Recording System

Analog Inputs

1. Control Column Angle

- 2. Throttle Command

3. Flap Command

4. Pitch Trim

5. Pitch Angle

6. Pitch Rate

7. Airspeed

8. Normal Acceleration

9. Control Wheel Angle

10. Foot Pedals

11. Yaw Angle

12. Sideslip Angle

13. Roll Angle

14. Roll Rate

15. Yaw Rate

16. Lateral Acceleration
17. Yaw Trim

18. Roll Trim

19. Elevator Deflection

20. Throttle setting

21. Flap Deflection

22. Axial Acceleration

23. Aileron Deflection

24. Rudder Deflection

25. VOR\#1 Azimuth

26. Angle of Attack

27. Barometric Altitude

28. Spare

29. MLS Azimuth

30. MLS Elevation

31. VOR\#2 Azimuth

32. Spare

\section{Digital Inputs}

1. DME Distance

2. VOR Frequency code

3. DME Frequency

4. Time

5. Response to Workload Lights

6. Spare

7. Spare

8. Spare

stalled in the ARA for another NASA program. Thus, there is a high degree of "plug compatibility" between the SPIFR and the DARE programs. A presampling filter (16 cps break-point 
Output Assignments for SPIFR Digital Data Recording system

\section{Analog Outputs}

1. Secondary workload meter \#1 4. Spare

2. Secondary workload meter \#2 5. Spare

3. Secondary workload meter \#3 6. Spare

\section{Digital Outputs}

1. DME tuning

2. DME station indicator.

3. Pilot workload lights
4. Avionics system status lights

5. Tape recorder

frequency) has been introduced for each analog channel to filter out the engine-vibration-induced noise.

Figure A.4 also illustrates the digital radio tuning feature that will be put to use during the next phase of the research. Error budget analyses, presented in Chapter 2, confirmed the superiority of DME over VOR for position fixing, even at the relatively-short ranges used in our flight 
tests. Consequently, it is advantageous to substitute multiple DME measurements for VOR measurements in flight data reduction. The BX-2000 DME unit can acquire and lock on a new station in less than one second; this feature has been used in DME-only "round-robin" position fixing for flight path determination.

The digital tape recording unit was the Hewlett Packard (HP) 2644 terminal, which housed two DCIOOA magnetic tape cartridge drive units. Its built-in memory enables transition from one cartridge to the other without losing any information. Such a pair of cartridges had a storage capability of about $220 \mathrm{~K}$ bytes, which was more than enough for a complete SPIFR mission run.

To accomodate the flight electronics, a pallet to fit into the ARA-aircraft behind the pilots' seats has been designed and built by the FRL technical staff. It weighs 215 $1 \mathrm{~b}$ and uses the same mounting brackets as the DARE pallet.

\section{A. 3 SOFTWARE DEVELOPMENT}

The SPIFR program focused on the low-frequency dynamic response of the airframe and on navigation-related information, whose rate of change is low as well. As discussed in 
Section 2.4, simulated SPIFR flight duration had to be about $30 \mathrm{~min}$, during which all the data channels had to be recorded at least once every second. Thus, the main objectives of the onboard software design were to:

* Sample the analog data at a high enough rate to avoid aliasing.

* Compress the high-frequency data so that the most significant flight test information could be recorded efficiently with minimal error.

* Trigger preprogrammed sequences of the secondary workload devices (lights, dummy meters).

* Enable the safety pilot to operate the data acquisition system via the hand-held CDU.

The information recorded in flight can be separated into "slow" and "fast" variables. The "slow" variables are principally the positional measurements, which can be sampled once per second with minimal aliasing effect. The "fast" variables, for example, angular rates and linear accelerations, are sampled ten times per second. For the sake of data compaction, they were averaged and recorded once each second. The simple averaging scheme is analogous to "lowpass" filtering. Thus, low-frequency information was passed with little modification, while high-frequency signals were attenuated. 
The HP 2644's recording format uses 16-bit binary words. The SBC $732 \mathrm{~A} / \mathrm{D}$ board is designed to fill in the 12 leftmost bit positions of a 16-bit field, and an appropriate shift is performed to comply with the standard output format of the HP 2644. Reference 72 contains additional details with regard to the software of the SPIFR onboard data acquisition system, plus the complete listing of the microprocessor Assembly program. 


\section{Appendix B \\ DERIVATION OF SPIFR CANDIDATE CRITERIA}

Following the definition of the SPIFR configurations (eq. (2-81) to (2-87)), the analytical frequency- and timedomain methods have to be applied to derive the candidate flying qualities criteria. Starting with the frequency domain, the phugoid natural frequency $\omega_{n p^{\prime}}$ damping $\zeta_{\mathrm{p}}$ and total damping $\left(\zeta \omega_{n}\right)_{p}$ parameters were computed for each configuration (Table B.1; the configuration codes are defined in Table 2.1). The values for configurations 2 to 7 are identical to the nominal values as they represent $G$ matrix variations, which do not affect the system's eigenvalues.

As may be observed from Table B.I, the total damping parameter provides the best differentiation between the configurations. Figure $B .1$ shows the results for $\left(\zeta \omega_{n}\right)_{p}$ in a histogram format.

Even for total damping the numerical values for thirteen out of fifteen configurations are in the narrow range of 0.04 to 0.07. Such clustering constitutes a disadvantage when applying the multiple regression algorithm. 
TABLE B.1

Natural Response Characteristics of the SPIFR Configurations

\begin{tabular}{|c|c|c|c|c|c|c|c|c|c|}
\hline $\begin{array}{c}\text { CONFIG- } \\
\text { URATION }\end{array}$ & 1 to 7 & 9 & 10 & 11 & 12 & 13 & 14 & 15 & 16 \\
\hline$\omega_{\mathrm{NP}} \frac{\mathrm{RAD}}{\mathrm{SEC}}$ & 0.34 & 0.38 & 0.28 & 0.29 & 0.38 & 0.34 & 0.33 & 0.35 & 0.33 \\
$\zeta_{\mathrm{P}}-$ & 0.16 & 0.18 & 0.14 & 0.16 & 0.16 & 0.034 & 0.29 & 0.16 & 0.16 \\
$\left(\zeta \omega_{\mathrm{N}}\right)_{\mathrm{P}} \frac{\mathrm{RAD}}{\mathrm{SEC}}$ & 0.054 & 0.067 & 0.040 & 0.047 & 0.060 & 0.011 & 0.096 & 0.056 & 0.052 \\
\hline
\end{tabular}

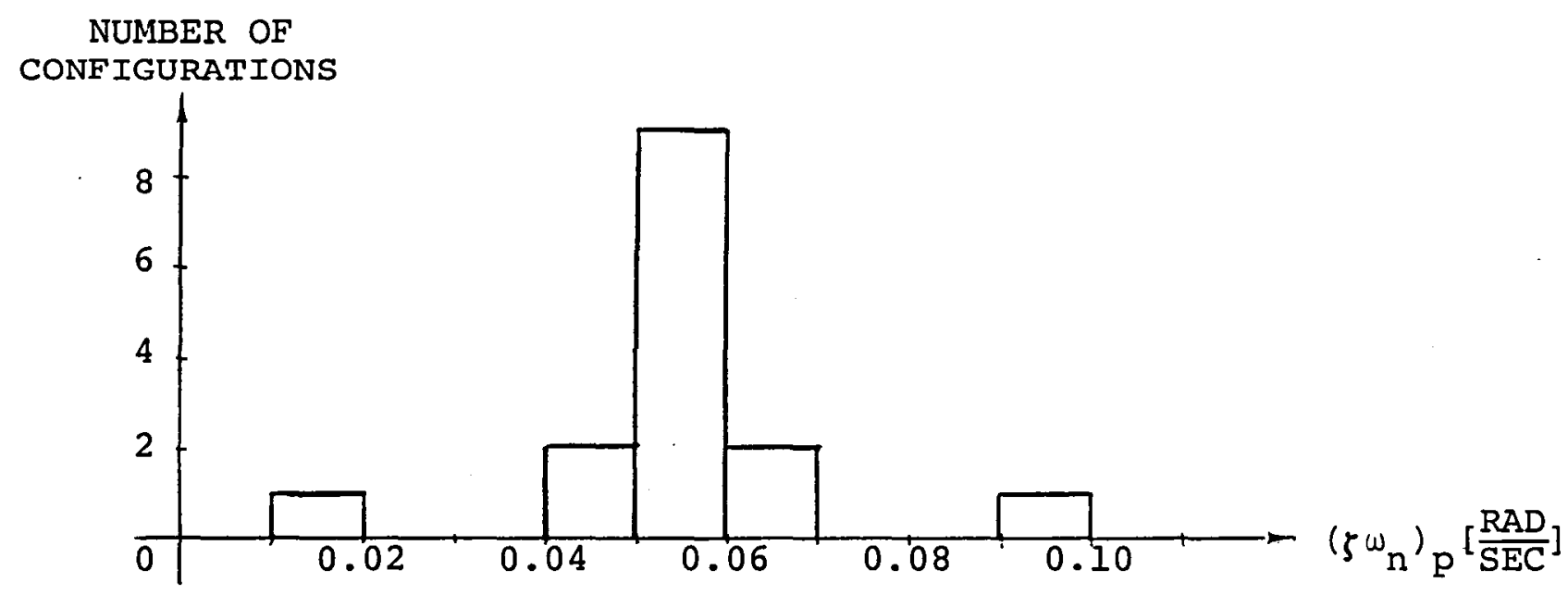

Figure B.1: Total Damping Histogram B-2 
Next, results of the output command algorithm application to the SPIFR configurations are summarized in Table B.2. They are given in terms of pitch attitude and control sensitivities to flight path angle and airspeed retrimming changes. The numerical values correspond to 4 -deg flight path angle retrimming (occurs, e.g., while leveling off from a 500-fpm rate-of-climb), and to a 10-kt airspeed retrimming, respectively. The pitch attitude sensitivity to airspeed retrimming was put in a histogram format in Fig. B.2. With regard to pitch attitude sensitivity to flight path angle changes, it is obvious directly from Table B.2 that it may not be a satsfactory configuration differentiator. One may observe from this table that the elevator sensitivities to airspeed and flight path angle changes have quite spread numerical values, as obvious from Fig. B.3 and B.4. To be used as general candidate criteria, they were multiplied by the

$$
\text { gearing }
$$

ratio,

$1.38 \mathrm{lb}$ stick force/deg of elevator deflection, to become $\Delta f_{s}^{*} / \Delta v_{\text {comm }}$ and $\Delta f_{s}^{*} / \Delta \gamma_{c o m m}$, respectively.

Finally, the transient component of the time-domain response was employed for the identification of the candidate criteria. First, the system was excited by steps of 1-deg elevator deflection or 108 throttle travel, and the consequent steady-state variations in the state variables were computed, using eq. $(2-74)$. Then, running the simulation 
TABLE B. 2

Output Command Algorithm Application to SPIFR Configurations

\begin{tabular}{|c|c|c|c|c|c|c|c|c|c|c|c|}
\hline \multicolumn{6}{|c|}{$\Delta \mathrm{V}_{\text {comm }}=10 \mathrm{KT}$} & \multicolumn{6}{|c|}{$\Delta \gamma_{\mathrm{comm}}=4 \mathrm{DEG}$} \\
\hline $\begin{array}{l}\text { CONFIG- } \\
\text { URATION }\end{array}$ & $\begin{array}{l}\Delta \theta^{\star} \\
D E G\end{array}$ & $\begin{array}{l}\text { CONFIG- } \\
\text { URATION }\end{array}$ & $\begin{array}{l}\Delta \delta E^{*} \\
D E G\end{array}$ & $\begin{array}{l}\text { CONFIG- } \\
\text { URATION }\end{array}$ & $\begin{array}{l}\Delta \delta T^{*} \\
z\end{array}$ & $\begin{array}{l}\text { CONFIG- } \\
\text { URATION }\end{array}$ & $\begin{array}{l}\Delta \theta^{\prime \prime} \\
D E G\end{array}$ & $\begin{array}{l}\text { CONFIG- } \\
\text { URATION }\end{array}$ & $\begin{array}{l}\Delta \delta E^{*} \\
D E G\end{array}$ & $\begin{array}{l}\text { CONFIG- } \\
\text { URATION }\end{array}$ & ${ }_{8}^{\Delta \delta T^{*}}$ \\
\hline $\begin{array}{c}1,2,3,9 \\
13,14\end{array}$ & -4.7 & 10,16 & 2.3 & 13 & -11.0 & $\mid \begin{array}{l}1,2,6,9 \\
12,13,14\end{array}$ & 3.8 & 3 & -1.0 & 5 & 11.7 \\
\hline 4 & -4.9 & 11 & 2.5 & 15 & -9.9 & $7,10,11$ & 3.9 & 4 & -0.5 & 15 & 12.5 \\
\hline 5,10 & -4.6 & 7 & 2.8 & 12 & -4.1 & 15 & 3.7 & 16 & 0.08 & 2 & 12.6 \\
\hline 6 & -5.1 & 2 & 3.2 & 6 & -2.5 & 16 & 3.9 & 7 & 0.10 & $\begin{array}{l}1,6,7 \\
9 \text { to } 14\end{array}$ & 12.8 \\
\hline 7 & -4.1 & 5 & 3.3 & 4,9 & -2.2 & 4 & 4.6 & 1,9 to 14 & 0.12 & 3,16 & 12.9 \\
\hline 11 & -3.7 & $1,13,14$ & 3.4 & $1,2,3$. & -2.0 & 5 & 3.3 & 6 & 0.13 & 4 & 14.1 \\
\hline 12 & -5.7 & 4 & 3.5 & 5,10 & -1.8 & 3 & 4.0 & 15 & 0.26 & & \\
\hline 15 & -9.1 & 3,6 & 3.6 & 7 & -0.8 & & & 5 & 0.6 & & \\
\hline 16 & -3.4 & 12 & 4.3 & 11 & -0.05 & & & 2 & 1.2 & & \\
\hline & & 9 & 4.5 & 16. & 0.03 & & & & & & \\
\hline & & 15 & 7.1 & 14 & 6.9 & & & & & & \\
\hline
\end{tabular}

from $t=0$ to $t=20 \mathrm{sec}$, for each case the rise time $\tau$ and absolute overshoot values $A$ for the $V$ and $\gamma$ output variables were computed. The convention here uses the flight path 
NUMBER OF

CONFIGURATIONS

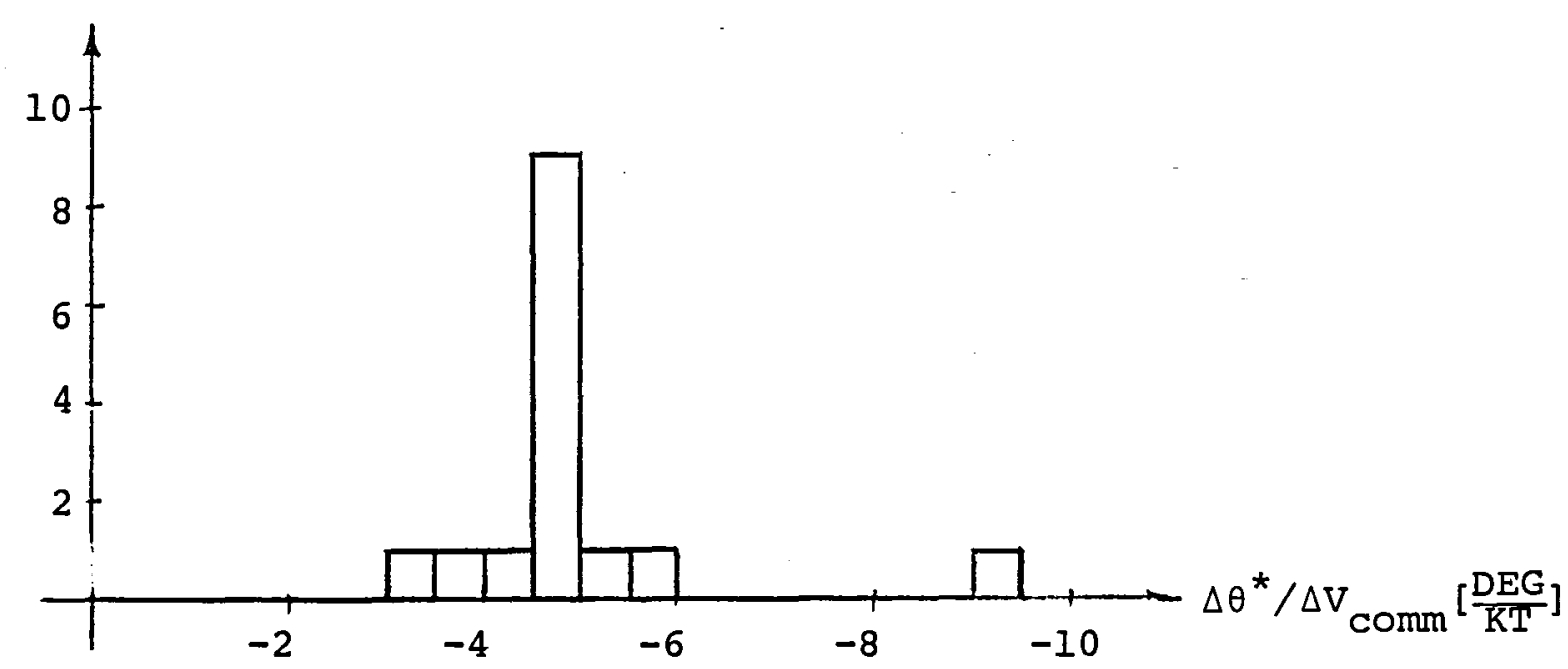

Figure B.2: Change in Pitch attitude for 10-kt Airspeed Retrimming

variable, for which $\tau$ or $A$ are specified; as subscript; the control variable that initiated the transient is given in parentheses. The results are summarized in Tables B.3 and B.4. Pitch rate overshoots also were computed but, as may have been expected, they may not provide distinct differentiation for phugoid-response-oriented configuration changes. The data of Tables B.3 and B.4 are given in a histogram format in Fig. B.5 to B.12. 
NUMBER OF

CONFIGURATIONS

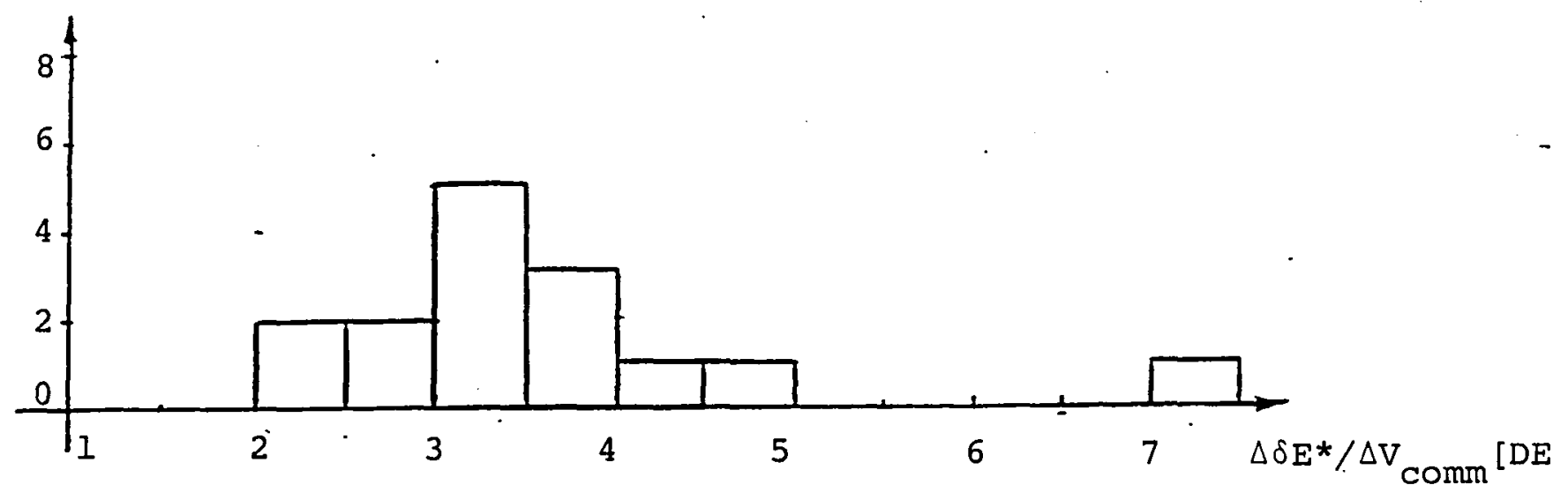

Figure B.3: Elevator Adjustment for 10-kt Airspeed Retrimming

The histograms in Figures B.l to B.12 provide a convenient indication of which of the candidate criteria are more likely to materialize as actual SPIFR flying qualities criteria, following the statistical analysis. For example, the values of the flight path angle overshoot for throttle input in Fig. B.12 are clustered for most of the configurations about the nominal and their overall variation range is only $1.5 \mathrm{deg}$. On the other hand, the values of the flight path angle rise time for throttle input in Fig. B.8, are more evenly spread over a significantly wide range of varia- 
NUMBER OF

CONF IGURATIONS

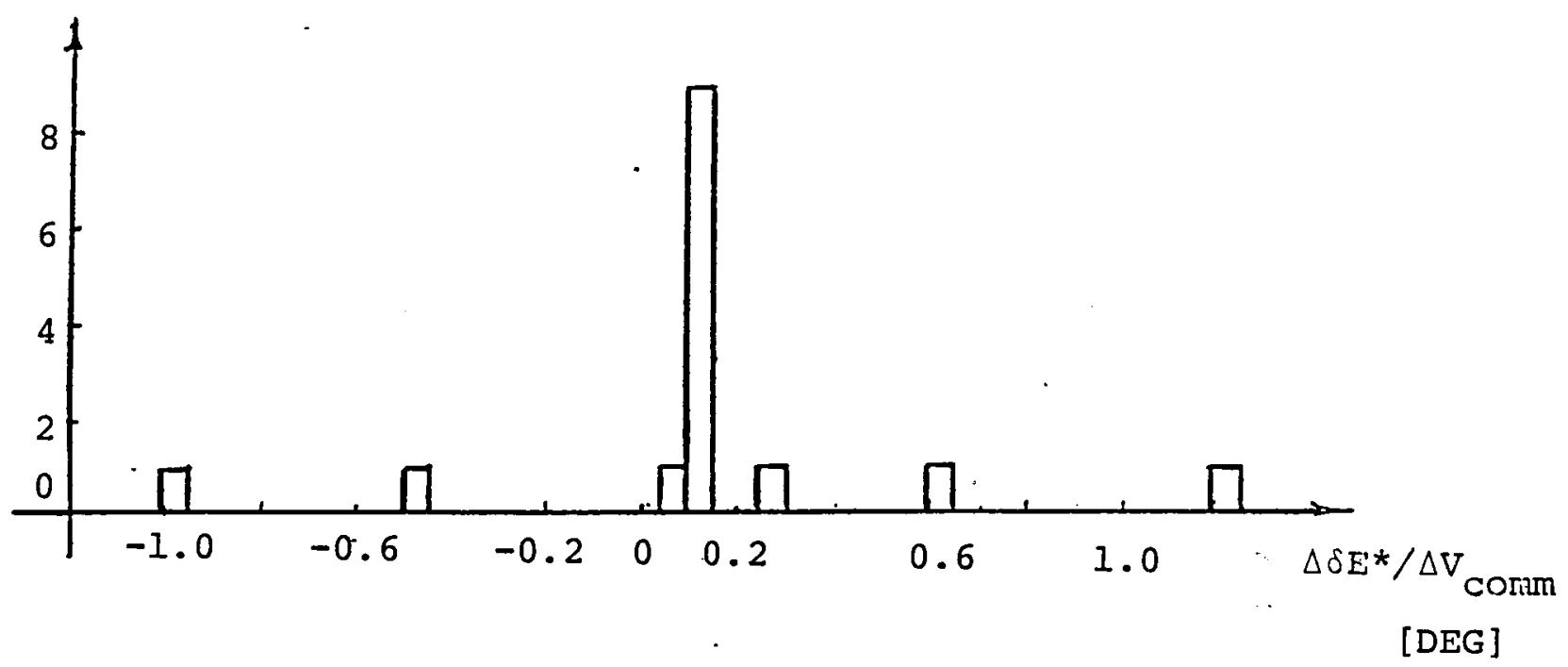

Figure B.4: Elevator Adjustment for 4-deg Flight Path Angle Retrimming

tion. As a result of these observations, the following dynamic response characteristics were used in the SPIFR statisical analysis: $\quad\left(\xi \omega_{n}\right)_{p^{\prime}} \quad \Delta \theta^{*} / \Delta v_{c o m m^{\prime}} \Delta f_{s}^{*} / \Delta v_{c o m m}{ }_{c o}$ $\Delta E_{S}^{*} / \Delta \gamma_{c o m m},{ }^{\tau}{ }_{V}(\delta E),{ }^{\top}{ }_{V}(\delta T),{ }^{\tau} \gamma(\delta T), A_{V}(\delta E), A_{\gamma}(\delta E), \quad$ and $A_{V}(\delta T)$. 
TABLE B. 3

Airspeed and Flight Path Angle Rise Time Values

\begin{tabular}{|c|c|c|c|c|c|c|c|}
\hline \multicolumn{4}{|c|}{$\Delta \delta \mathrm{E}_{\mathrm{comm}}=1 \quad[\mathrm{DEG}]$} & \multicolumn{4}{|c|}{$\Delta \delta T_{\text {comm }}=10 \%$} \\
\hline $\begin{array}{c}\text { CONFIGUR- } \\
\text { ATION }\end{array}$ & {$\left[\begin{array}{c}\tau_{\mathrm{V}} \\
{[\mathrm{SEC}]}\end{array}\right.$} & $\begin{array}{c}\text { CONF IGUR- } \\
\text { ATION }\end{array}$ & $\begin{array}{l}\tau_{\gamma}^{\gamma} \\
{[\text { SEC] }}\end{array}$ & $\begin{array}{c}\text { CONFIGUR- } \\
\text { ATION }\end{array}$ & $\stackrel{\tau}{\mathrm{V}} \mathrm{V}^{\mathrm{S}}$ & $\begin{array}{c}\text { CONFIGURA- } \\
\text { TION }\end{array}$ & $\left.\begin{array}{c}\tau \\
\gamma \\
S E C\end{array}\right]$ \\
\hline 15 & 3.75 & 13 & 0.38 & 9,12 & 0.25 & 2 & 3.5 \\
\hline $9,12,13$ & 4.0 & $\begin{array}{ll}1 \text { to } 7,9 \\
12,15\end{array}$ & 0.75 & $\begin{array}{c}1,6,7,10, \\
11,13 \text { to } \\
16\end{array}$ & 0.5 & 5 & 4.5 \\
\hline 1 to 7 & 4.5 & 11,16 & 1.0 & 4 & 1.0 & $9,12,13$ & 5.0 \\
\hline 14,16 & 5.0 & 10 & 1.25 & 3 & 2.0 & $1,6,7,15$, & 6.0 \\
\hline 10,11 & 5.5 & 14 & 1.5 & 2 & 8.0 & $4,10,11,14$ & 7.0 \\
\hline & & & & 5 & 9.0 & 3 & 8.0 \\
\hline
\end{tabular}


TABLE B. 4

Airspeed and Flight Path Angle Overshoot Values

\begin{tabular}{|c|c|c|c|c|c|c|c|}
\hline \multicolumn{4}{|c|}{$\Delta \delta E_{\text {comm }}=1$ [DEG] } & \multicolumn{4}{|c|}{$\Delta \delta \mathbf{T}_{\mathrm{comm}}=10 \%$} \\
\hline $\begin{array}{l}\text { CONEIGUR- } \\
\text { ATION \# }\end{array}$ & $\begin{array}{l}\mathrm{A}_{\mathrm{V}} \\
\text { [FPS }\end{array}$ & $\begin{array}{l}\text { CONFIGUR- } \\
\text { ATION \# }\end{array}$ & $\begin{array}{l}\mathrm{A}_{\mathrm{Y}} \\
{[\mathrm{DEG}]}\end{array}$ & $\begin{array}{l}\text { CONFIGUR- } \\
\text { ATION \# }\end{array}$ & $\begin{array}{l}\mathrm{A}_{\mathrm{V}} \\
\text { [FPS] }\end{array}$ & $\begin{array}{l}\text { CONFIGUR- } \\
\text { ATION \# }\end{array}$ & $\begin{array}{c}\mathrm{A} Y \\
\text { [DEG] }\end{array}$ \\
\hline 15 & 1.7 & 15 & 1.3 & 14 & 2.8 & 14 & 1.3 \\
\hline 14 & 2.2 & 14 & 1.9 & 9 & 2.9 & 9 & 1.8 \\
\hline 9 & 2.4 & 9 & 2.1 & 5,12 & 3.0 & $\begin{array}{l}1,4,6,7,11 \\
12,15,16\end{array}$ & 1.9 \\
\hline 12 & 2.6 & 12 & 2.2 & 15 & 3.1 & 10 & 2.0 \\
\hline 6 & 3.1 & 6 & 2.3 & $1,6,7$ & 3.3 & 5 & 2.2 \\
\hline 1 to 5 & 3.3 & 1 to 5 & 2.5 & 16 & 3.45 & 3 & 2.3 \\
\hline 7 & 3.9 & 11 & 2.8 & 11 & 3.6 & 2,13 & 2.8 \\
\hline 11 & 4.3 & 7 & 2.9 & 2 & 3.74 & & \\
\hline 16 & 4.7 & 10,13 & 3.1 & 10 & 3.9 & & \\
\hline 13 & 4.8 & 16 & 3.4 & 4 & 4.1 & & \\
\hline 10 & 5.0 & & & 13 & 4.3 & & \\
\hline & & & & 3 & 5.0 & & \\
\hline
\end{tabular}




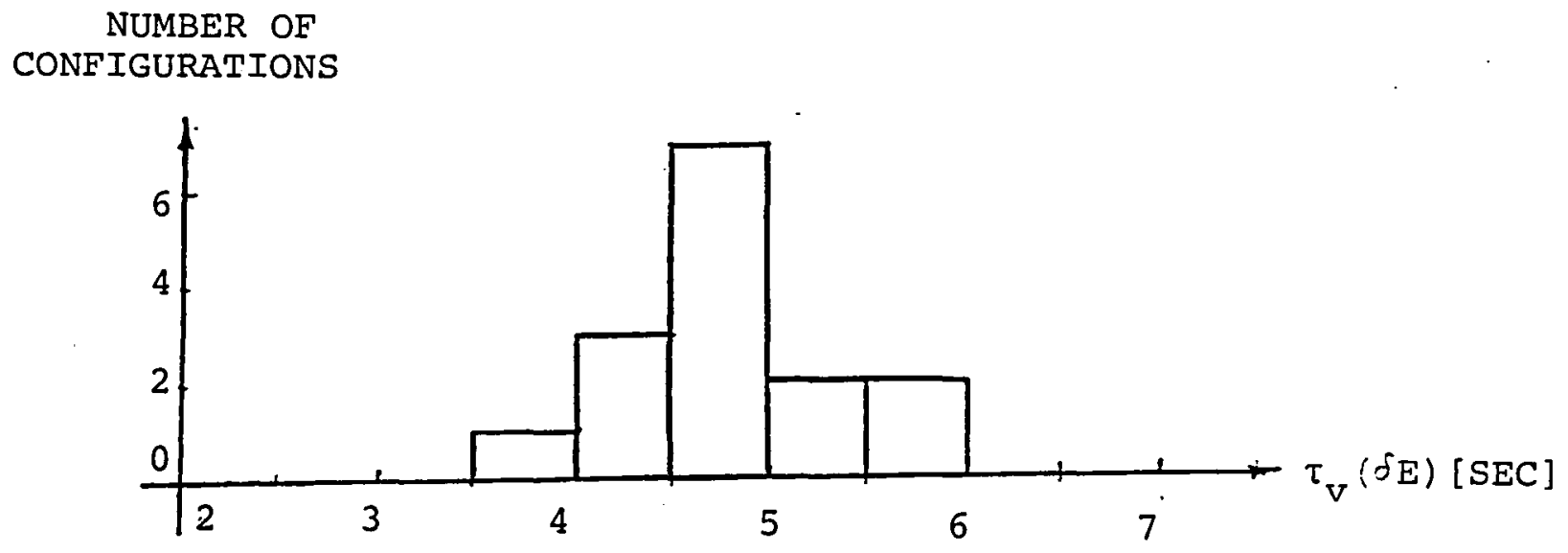

Figure B.5: Airspeed Rise Time for 1-deg Elevator step Input

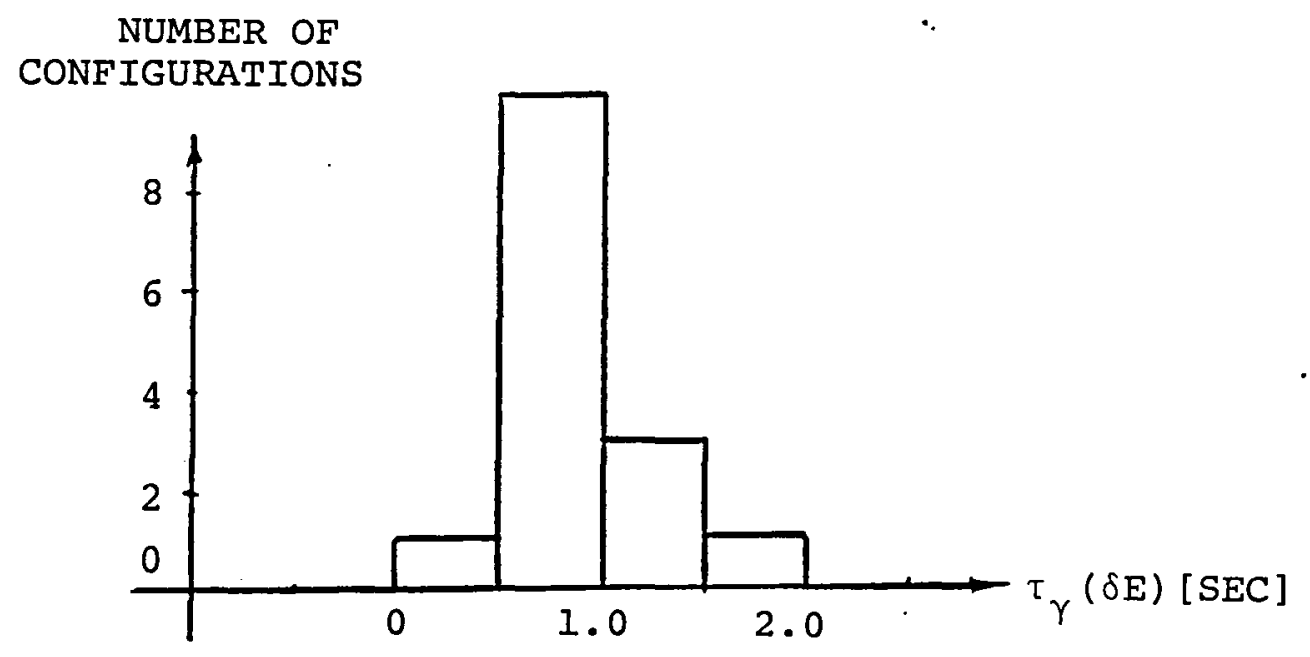

Figure B.6: Flight Path Angle Rise Time for 1-deg Elevator Step Input 


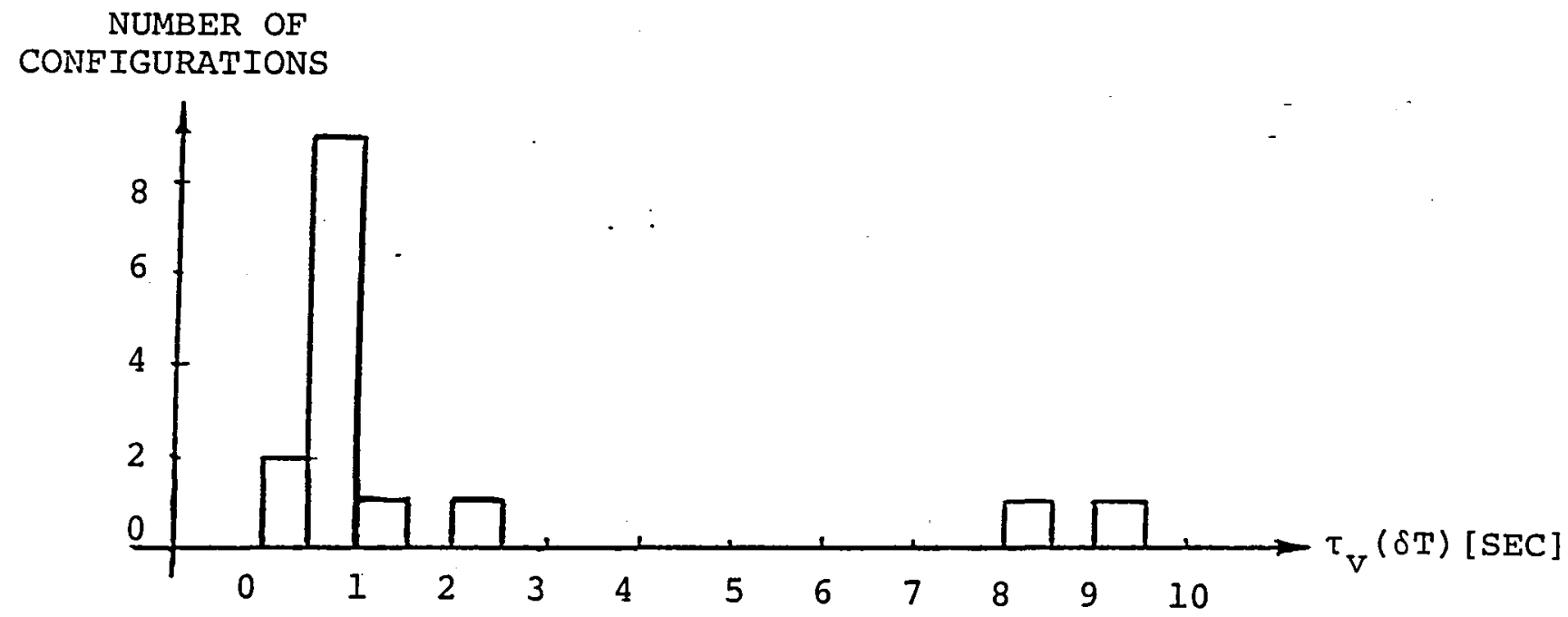

Figure B.7: Airspeed Rise Time for 108 Throttle Step Input

NUMBER OF

CONF IGURATIONS

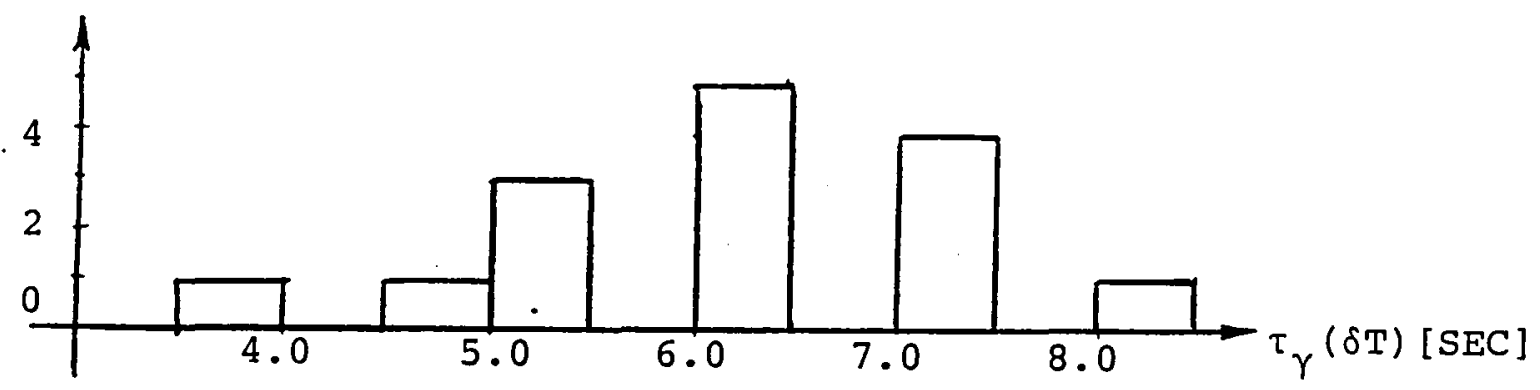

Figure B.8: Flight Path Angle Rise Time for 108 Throttle Step Input 


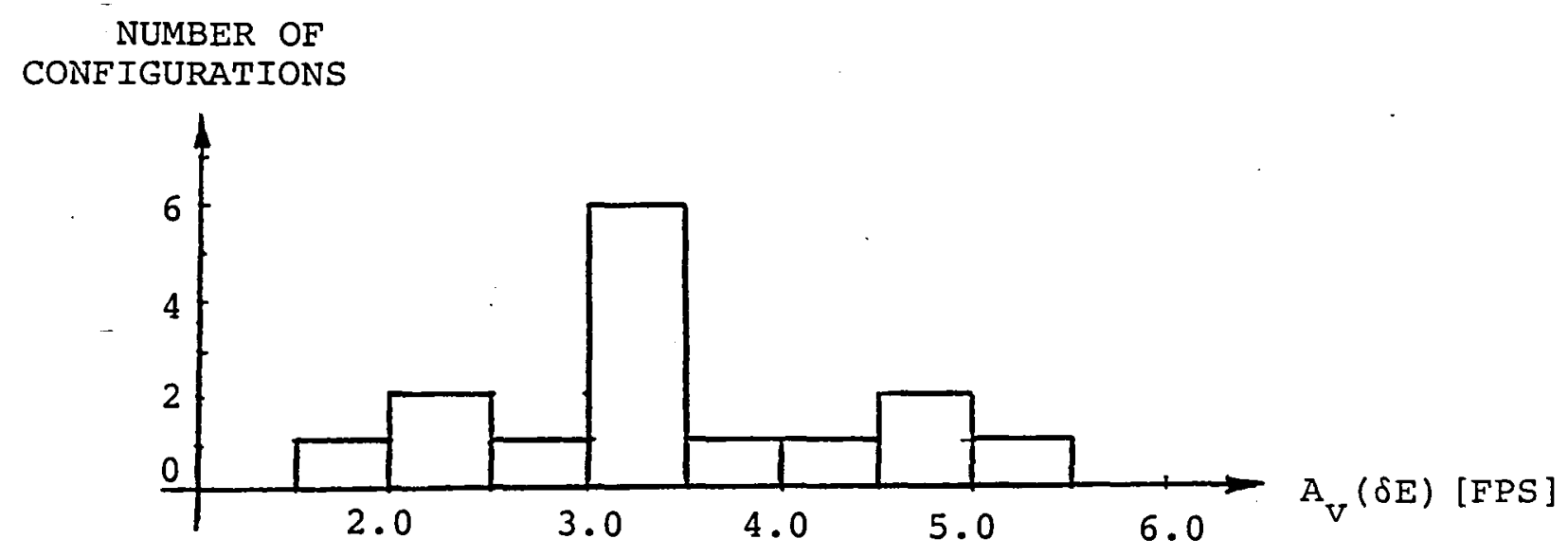

Figure B.9: Airspeed Overshoot for 1-deg Elevator Step Input

NUMBER OF

CONFIGURATIONS

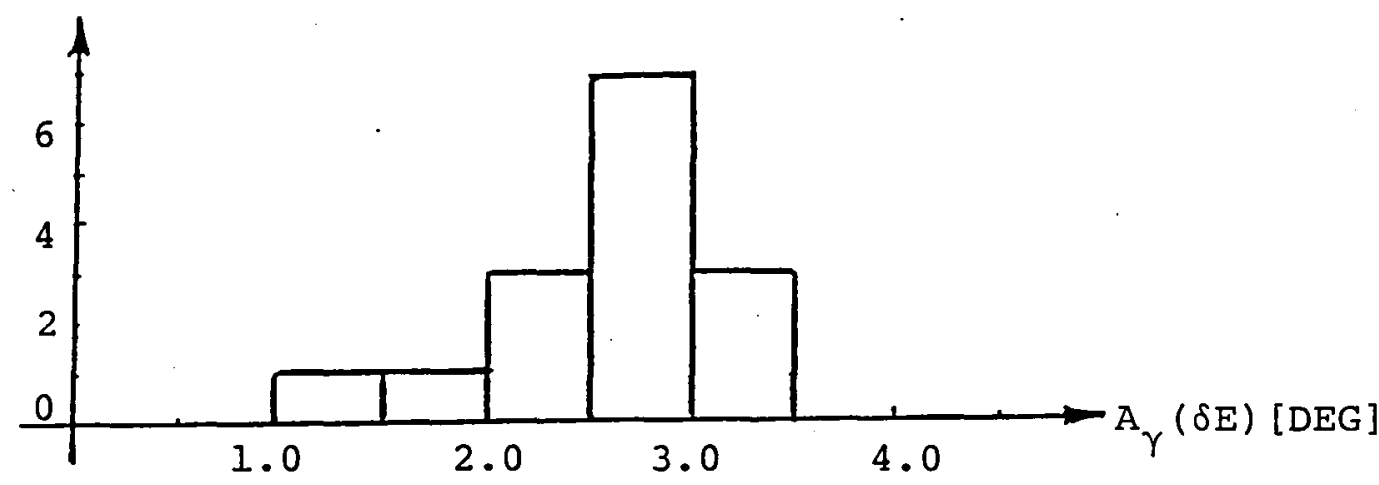

Figure B.10: Flight Path Angle Overshoot for 1-deg Elevator Step Input 
NUMBER OF

CONFIGURATIONS

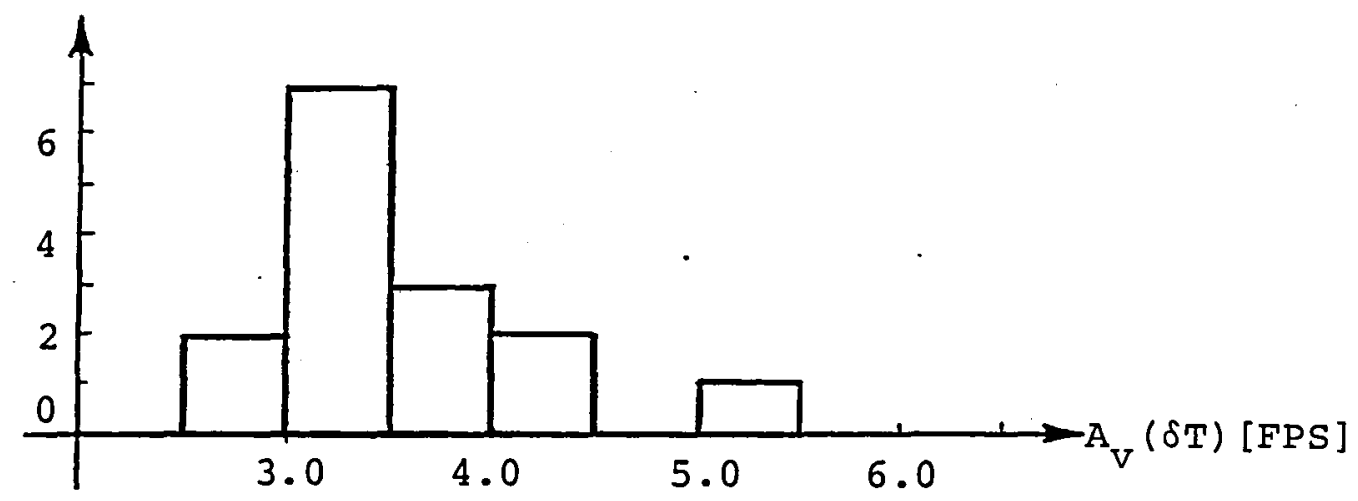

Figure B.Il: Airspeed Overshoot for $10 \%$ Throttle Step Input

NUMBER OF

CONFIGURATIONS

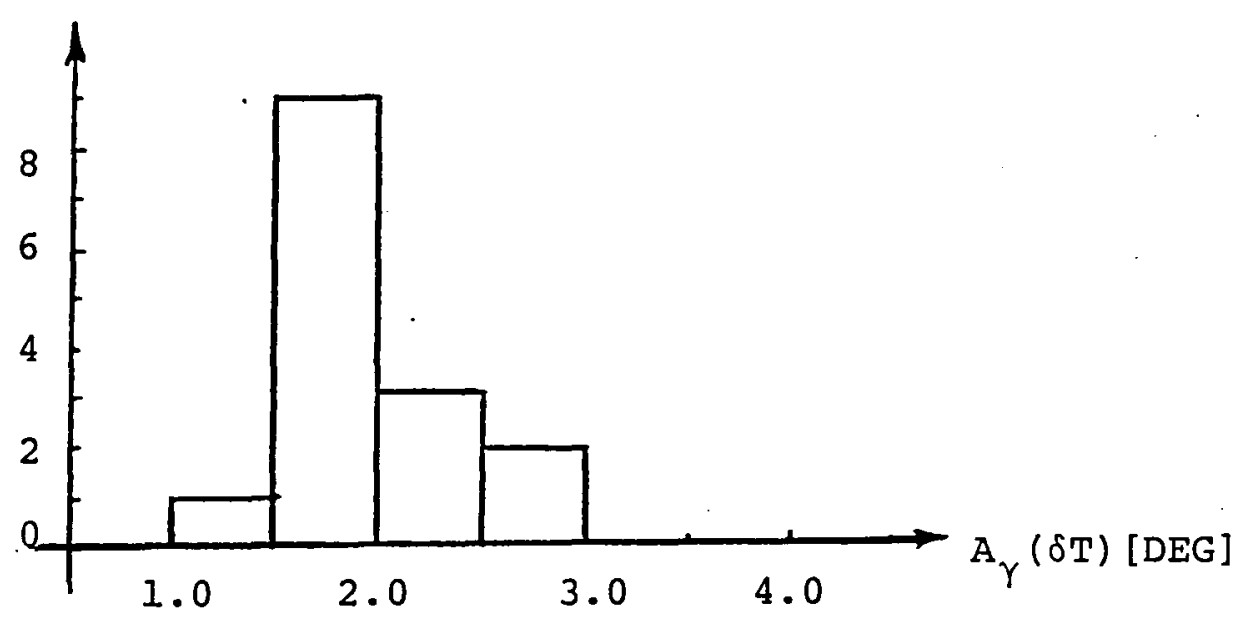

Figure B.12: Flight Path Angle Overshoot for 108 Throttle Step Input 


\section{Appendix C \\ LINEARIZATION OF THE SPIFR MATHEMATICAI MODEL}

\section{1 LINEARIZATION OF MODEL $\underline{A}$}

Model A is given in equations (3-22) to (3-27). Analytical derivation of state matrix $\mathrm{F}_{\mathrm{A}}$ renders,

$$
\dot{x}_{A}=\left[\begin{array}{c}
\dot{p} \\
\dot{q} \\
\dot{r} \\
\dot{\phi} \\
\dot{\theta} \\
\dot{\psi} \\
\dot{\mathrm{b}}_{\psi}
\end{array}\right]=\mathrm{F}_{\mathrm{A}}\left[\begin{array}{c}
\mathrm{p} \\
\mathrm{q} \\
\mathrm{r} \\
\phi \\
\theta \\
\psi \\
\mathrm{b}_{\psi}
\end{array}\right]+\underline{w}_{\mathrm{A}}
$$

where
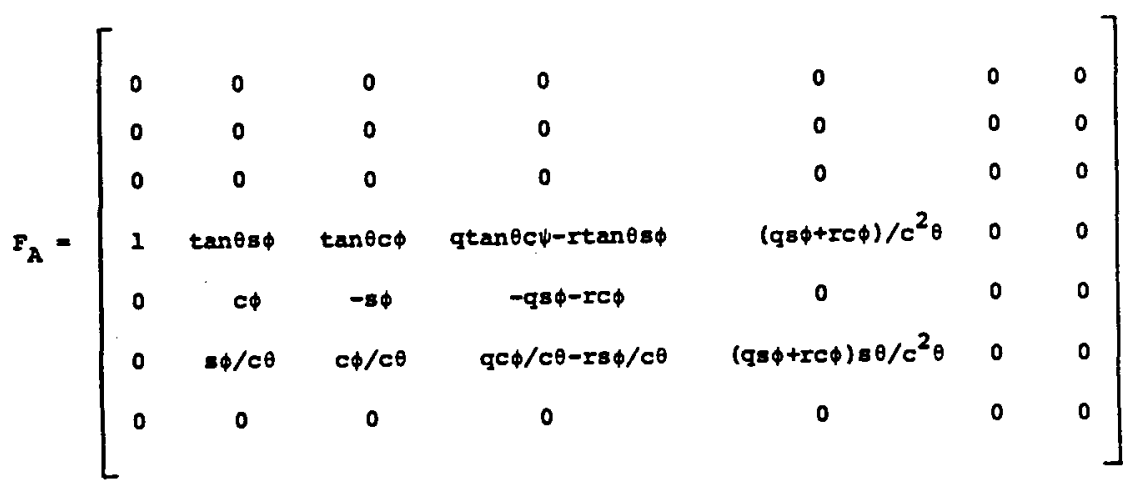
The measurement equation $(3-27)$ is linear.

\section{C.2 LINEARIZATION OF MODEL B}

Model $B$ is given by equations $(3-28)$ and $(3-29)$ plus the associated definitions. The analytical derivation of state matrix $F_{B}$ preserves the structure of "flat Earth" approximation plus "correction" terms $c_{i j}$ '

$$
\dot{\underline{x}}_{B}=\left[\begin{array}{c}
\dot{x}_{c} \\
\dot{y}_{c} \\
\dot{z}_{c} \\
\dot{u} \\
\dot{v} \\
\dot{w} \\
\dot{w}_{x} \\
\dot{w}_{y} \\
\dot{w}_{z} \\
\dot{b}_{s 1} \\
\dot{b}_{s 2}
\end{array}\right]=F_{B}\left[\begin{array}{c}
x_{c} \\
y_{c} \\
z_{c} \\
u \\
v \\
w \\
w_{x} \\
w_{y} \\
w_{z} \\
b_{s 1} \\
b_{s 2}
\end{array}\right]+\left[\begin{array}{c}
0 \\
0 \\
0 \\
a_{x}-g s \theta \\
a_{y}+g c \theta s \phi \\
a_{z}+g c \theta c \phi \\
0 \\
0 \\
0 \\
0 \\
0
\end{array}\right]+w_{B}
$$

where 


$$
F_{B}=\left[\begin{array}{ccccccccccc}
c_{11} & 0 & c_{13} & H_{B 11}^{I}+c_{14} & H_{B 12}^{I}+c_{15} & H_{B 13}^{I}+c_{16} & 1+c_{17} & 0 & 0 & 0 & 0 \\
c_{21} & c_{22} & c_{23} & H_{B 21}^{I}+c_{24} & H_{B 22}^{I}+c_{25} & H_{B 23}^{I}+c_{26} & c_{27} & 1+c_{28} & 0 & 0 & 0 \\
c_{31} & 0 & c_{33} & H_{B 31}^{I}+c_{34} & H_{B 32}^{I}+c_{35} & H_{B 33}^{I}+c_{36} & c_{37} & 0 & 1 & 0 & 0 \\
c_{41} & 0 & c_{43} & 0 & +r+c_{54} & -q+c_{46} & c_{47} & c_{48} & c_{49} & 0 & 0 \\
c_{51} & 0 & c_{53} & -r+c_{54} & 0 & +p+c_{56} & c_{57} & c_{58} & c_{59} & 0 & 0 \\
c_{61} & 0 & c_{63} & +q+c_{64} & -p+c_{65} & 0 & c_{67} & c_{68} & c_{69} & 0 & 0 \\
0 & 0 & 0 & 0 & 0 & 0 & 0 & 0 & 0 & 0 & 0 \\
0 & 0 & 0 & 0 & 0 & 0 & 0 & 0 & 0 & 0 & 0 \\
0 & 0 & 0 & 0 & 0 & 0 & 0 & 0 & 0 & 0 & 0 \\
0 & 0 & 0 & 0 & 0 & 0 & 0 & 0 & 0 & 0 & 0 \\
0 & 0 & 0 & 0 & 0 & 0 & 0 & 0 & 0 & 0 & 0
\end{array}\right] \text { (C-4) }
$$

$$
\begin{aligned}
& c_{11}=-\varepsilon^{2} \sin 2\left(\lambda_{0}+\lambda\right) c_{111} /(1-\gamma)^{2} \\
& c_{21}=-\varepsilon^{2} \sin 2\left(\lambda_{0}+\lambda\right) c_{211} /(1-\gamma)^{2}+c_{111}[-(1-\gamma) \\
& \left.+0.5 \varepsilon^{2} \sin ^{2} 2\left(\lambda_{0}+\lambda\right)\right] y_{c} / a /\left[(1-\gamma) \cos \left(\lambda_{\circ}+\lambda\right)\right]^{2} \\
& c_{31}=\varepsilon^{2} c_{111}\left[2 \cos 2\left(\lambda_{0}+\lambda\right)-\varepsilon^{2} \sin ^{2} 2\left(\lambda_{0}+\lambda\right) /(1-\gamma)\right] /(1-\gamma)(c-7) \\
& c_{22}=-c_{111} \tan \left(\lambda_{0}+\lambda\right) /(1-\gamma) \\
& c_{13}=+c_{111} /(1-\gamma)^{2} \\
& c_{23}=+c_{211} /(1-\gamma)^{2}-y_{c} c_{13} \tan \left(\lambda_{0}+\lambda\right) / a \\
& c_{33}=+\varepsilon^{2} c_{13} \sin 2\left(\lambda_{0}+\lambda\right)
\end{aligned}
$$


where, referring to Chapter 3 and defining intermediate variables,

$$
\begin{aligned}
& \gamma \triangleq 1-\left(-z_{C}+\rho_{G}\right) / a \\
& \left.\begin{array}{l}
\rho_{G}=\left[1-0.5 \varepsilon^{2} \cos 2\left(\lambda_{0}+\lambda\right)\right] \\
\varepsilon^{2}=0.0067
\end{array}\right\} \\
& (3-13) \\
& a=2.094 \times 10^{7} \mathrm{ft} \\
& (3-11) \\
& c_{111}=\left(\mathrm{H}_{\mathrm{B} 11}^{\mathrm{I}} \mathrm{u}+\mathrm{H}_{\mathrm{B} 12}^{\mathrm{I}} \mathrm{v}+\mathrm{H}_{\mathrm{B} 13}^{\mathrm{I}} \mathrm{w}+\mathrm{w}_{\mathrm{x}}\right) / \mathrm{a} \\
& (c-12) \\
& c_{211}=\left(\mathrm{H}_{\mathrm{B} 21}^{\mathrm{I}} \mathrm{u}+\mathrm{H}_{\mathrm{B} 22}^{\mathrm{I}} \mathrm{v}+\mathrm{H}_{\mathrm{B} 23^{\mathrm{w}}}^{\mathrm{I}}+\mathrm{w}_{\mathrm{y}}\right) / \mathrm{a} \\
& (C-13)
\end{aligned}
$$

Continuing,

$$
\begin{aligned}
& c_{14}=c_{141} \mathrm{H}_{\mathrm{B} 11}^{\mathrm{I}} \\
& c_{15}=c_{141} \mathrm{H}_{\mathrm{B} 12}^{\mathrm{I}} \\
& c_{16}=c_{141} \mathrm{H}_{\mathrm{B} 13}^{\mathrm{I}} \\
& c_{24}=c_{241} \mathrm{H}_{\mathrm{B} 11}^{\mathrm{I}}+c_{141} \mathrm{H}_{\mathrm{B} 21}^{\mathrm{I}} \\
& c_{25}=c_{241} \mathrm{H}_{\mathrm{B} 12}^{\mathrm{I}}+c_{141} \mathrm{H}_{\mathrm{B} 22}^{\mathrm{I}} \\
& c_{26}=c_{241} \mathrm{H}_{\mathrm{B} 13}^{\mathrm{I}}+c_{141} \mathrm{H}_{\mathrm{B} 23}^{\mathrm{I}} \\
& c_{34}=c_{341} \mathrm{H}_{\mathrm{B} 11}^{\mathrm{I}} \\
& c_{35}=c_{341} \mathrm{H}_{\mathrm{B} 12}^{\mathrm{I}} \\
& c_{36}=c_{341} \mathrm{H}_{\mathrm{B} 13}^{\mathrm{I}}
\end{aligned}
$$

$(\mathrm{C}-14)$

$(\mathrm{C}-15)$

$(\mathrm{C}-16)$

$(\mathrm{c}-17)$

(c-18)

(c-19)

(c-20)

$(C-21)$

$(c-22)$

C-4 


$$
\begin{aligned}
& c_{17}=c_{141} \\
& c_{27}=c_{241} \\
& c_{28}=c_{141} \\
& c_{37}=c_{341}
\end{aligned}
$$

where

$$
\begin{aligned}
& c_{141}=\left[z_{c} / a+0.5 \varepsilon^{2} \cos 2\left(\lambda_{o}+\lambda\right)\right] /(1-\gamma) \\
& c_{241}=-y_{c} \tan \left(\lambda_{o}+\lambda\right) / a /(1-\gamma) \\
& c_{341}=\varepsilon^{2} \sin 2\left(\lambda_{o}+\lambda\right) /(1-\gamma)
\end{aligned}
$$

Continuing,

$$
\begin{aligned}
& c_{41}=\left(c_{412} \mathrm{w}-c_{411} v\right) / a-c_{4111} \mathrm{H}_{\mathrm{I} 11}^{\mathrm{B}}+c_{4112} \mathrm{H}_{\mathrm{I} 13}^{\mathrm{B}}+\left[\cos \left(\lambda_{\circ}+\lambda\right)\right. \\
& \left.\left(w_{x} H_{I 12}^{B}-w_{y} H_{I 11}^{B}\right)+\sin \left(\lambda_{0}+\lambda\right)\left(w_{y} H_{I 13}^{B}+w_{z} H_{I 12}^{B}\right)\right] \Omega / a \\
& c_{51}=\left(c_{411} \mathrm{u}-c_{413} w\right) / a-c_{4111} \mathrm{H}_{I 21}^{\mathrm{B}}+c_{4112^{\mathrm{H}}}^{\mathrm{B}}{ }_{I 23}+\left[\cos \left(\lambda_{0}+\lambda\right)\right. \\
& \left.\left(w_{x} H_{I 22}^{B}-W_{y} H_{I 21}^{B}\right)+\sin \left(\lambda_{0}+\lambda\right)\left(w_{y} H_{I 23}^{B}+w_{z} H_{I 22}^{B}\right)\right] \Omega / a \\
& c_{61}=\left(c_{413^{v}} v-c_{412} u\right) / a-c_{4111} H_{I 31}^{B}+c_{4112^{2}} H_{I 33}^{B}+\left[\cos \left(\lambda_{\circ}+\lambda\right)\right. \\
& \left.\left(W_{x} H_{I 32}^{B}-W_{y} H_{I 31}^{B}\right)+\sin \left(\lambda_{0}+\lambda\right)\left(w_{y} H_{I 33}^{B}+W_{z} H_{I 32}^{B}\right)\right] \Omega / a
\end{aligned}
$$

where

$$
\begin{gathered}
c_{411}=\Omega\left[\mathrm{H}_{I 31}^{\mathrm{B}} \sin \left(\lambda_{0}+\lambda\right)+\mathrm{H}_{I 33}^{\mathrm{B}} \cos \left(\lambda_{0}+\lambda\right)\right] \\
\mathrm{c}_{412}=\Omega\left[\mathrm{H}_{\mathrm{I} 21}^{\mathrm{B}} \sin \left(\lambda_{0}+\lambda\right)+\mathrm{H}_{\mathrm{I} 23}^{\mathrm{B}} \cos \left(\lambda_{0}+\lambda\right)\right] \\
\mathrm{c}-5
\end{gathered}
$$




$$
\begin{aligned}
& \mathrm{c}_{413}=\Omega\left[\mathrm{H}_{\mathrm{I} 11}^{\mathrm{B}} \sin \left(\lambda_{\mathrm{O}}+\lambda\right)+\mathrm{H}_{\mathrm{I} 13}^{\mathrm{B}} \cos \left(\lambda_{\mathrm{o}}+\lambda\right)\right] \quad(\mathrm{C}-35) \\
& \mathrm{c}_{4111}=\Omega^{2}\left[(1-\gamma) \cos 2\left(\lambda_{\mathrm{o}}+\lambda\right)+0.5 \varepsilon^{2} \sin ^{2} 2\left(\lambda_{\circ}+\lambda\right)\right] \quad(\mathrm{C}-36) \\
& \mathrm{c}_{4112}=\Omega^{2}\left[(1-\gamma) \sin 2\left(\lambda_{\mathrm{o}}+\lambda\right)-\varepsilon^{2} \sin 2\left(\lambda_{\mathrm{o}}+\lambda\right) \cos ^{2}\left(\lambda_{\mathrm{o}}+\lambda\right)\right](\mathrm{C}-37) \\
& \left.\Omega=0.728 \times 10^{-4} \mathrm{rad} / \mathrm{sec} \quad \text { (Section } 3.1\right)
\end{aligned}
$$

Continuing,

$$
\begin{aligned}
& c_{43}=\Omega c_{413} \cos \left(\lambda_{0}+\lambda\right) \\
& c_{53}=\Omega c_{412} \cos \left(\lambda_{0}+\lambda\right) \\
& c_{63}=\Omega c_{411} \cos \left(\lambda_{0}+\lambda\right) \\
& c_{56}=\Omega\left[\mathrm{H}_{I 11}^{\mathrm{B}} \cos \left(\lambda_{0}+\lambda\right)-\mathrm{H}_{\mathrm{I} 13}^{\mathrm{B}} \sin \left(\lambda_{0}+\lambda\right)\right] \\
& c_{65}=-c_{56} \\
& \mathrm{c}_{64}=\Omega\left[\mathrm{H}_{\mathrm{I} 21}^{\mathrm{B}} \cos \left(\lambda_{0}+\lambda\right)-\mathrm{H}_{\mathrm{I} 23}^{\mathrm{B}} \sin \left(\lambda_{0}+\lambda\right)\right] \\
& c_{46}=-c_{64} \\
& c_{45}=\Omega\left[H_{I 31}^{B} \cos \left(\lambda_{0}+\lambda\right)-H_{I 33}^{B} \sin \left(\lambda_{0}+\lambda\right)\right] \\
& c_{54}=-c_{45} \\
& c_{47}=2\left(\mathrm{rH}_{\mathrm{I} 21}^{\mathrm{B}}-\mathrm{qH}_{\mathrm{I} 31}^{\mathrm{B}}\right)+\Omega \mathrm{H}_{\mathrm{I} 12}^{\mathrm{B}} \sin \left(\lambda_{0}+\lambda\right)^{-} \\
& \mathrm{c}_{48}=2\left(\mathrm{rH}_{\mathrm{I} 22}^{\mathrm{B}}-\mathrm{qH}_{\mathrm{I} 32}^{\mathrm{B}}\right)-\mathrm{c}_{413} \\
& c_{49}=2\left(\mathrm{rH}_{\mathrm{I} 23}^{\mathrm{B}}-\mathrm{qH}_{\mathrm{I} 33}^{\mathrm{B}}\right)-\Omega \mathrm{H}_{\mathrm{I} 12}^{\mathrm{B}} \cos \left(\lambda_{0}+\lambda\right) \\
& c_{57}=2\left(-\mathrm{rH}_{\mathrm{I} 11}^{\mathrm{B}}+\mathrm{qH}_{\mathrm{I} 31}^{\mathrm{B}}\right)+\Omega \mathrm{H}_{\mathrm{I} 22}^{\mathrm{B}} \sin \left(\lambda_{0}+\lambda\right) \\
& \text { C-6 }
\end{aligned}
$$




$$
\begin{aligned}
& \mathrm{c}_{58}=2\left(-\mathrm{rH}_{\mathrm{I} 12}^{\mathrm{B}}+\mathrm{pH}_{\mathrm{I} 32}^{\mathrm{B}}\right)-\mathrm{c}_{412} \\
& \mathrm{c}_{59}=2\left(-\mathrm{rH}_{\mathrm{I} 13}^{\mathrm{B}}+\mathrm{pH}_{\mathrm{I} 33}^{\mathrm{B}}\right)+\Omega \mathrm{H}_{\mathrm{I} 22}^{\mathrm{B}} \cos \left(\lambda_{\circ}+\lambda\right) \\
& \mathrm{c}_{67}=2\left(+\mathrm{qH}_{\mathrm{I} 11}^{\mathrm{B}}-\mathrm{pH}_{\mathrm{I} 21}^{\mathrm{B}}\right)+\Omega \mathrm{H}_{\mathrm{I} 32}^{\mathrm{B}} \sin \left(\lambda_{\circ}+\lambda\right) \\
& \mathrm{c}_{68}=2\left(+\mathrm{qH}_{\mathrm{I} 12}^{\mathrm{B}}-\mathrm{pH}_{\mathrm{I} 22}^{\mathrm{B}}\right)-\mathrm{c}_{411} \\
& \mathrm{c}_{69}=2\left(+\mathrm{qH}_{\mathrm{I} 13}^{\mathrm{B}}-\mathrm{pH}_{\mathrm{I} 23}^{\mathrm{B}}\right)+\Omega \mathrm{H}_{\mathrm{I} 32}^{\mathrm{B}} \cos \left(\lambda_{\circ}+\lambda\right)
\end{aligned}
$$

The linearized measurement equation is,

$$
\underline{z}_{B}=\left[\begin{array}{c}
v \\
\alpha \\
h \\
r_{s 1} \\
r_{s 2}
\end{array}\right]=H_{B}\left[\begin{array}{l}
x_{C} \\
y_{c} \\
z_{c} \\
v \\
w \\
w_{x} \\
w_{y} \\
w_{z} \\
b_{s 1} \\
b_{s 2}
\end{array}\right]+\underline{v}_{B}
$$

where 


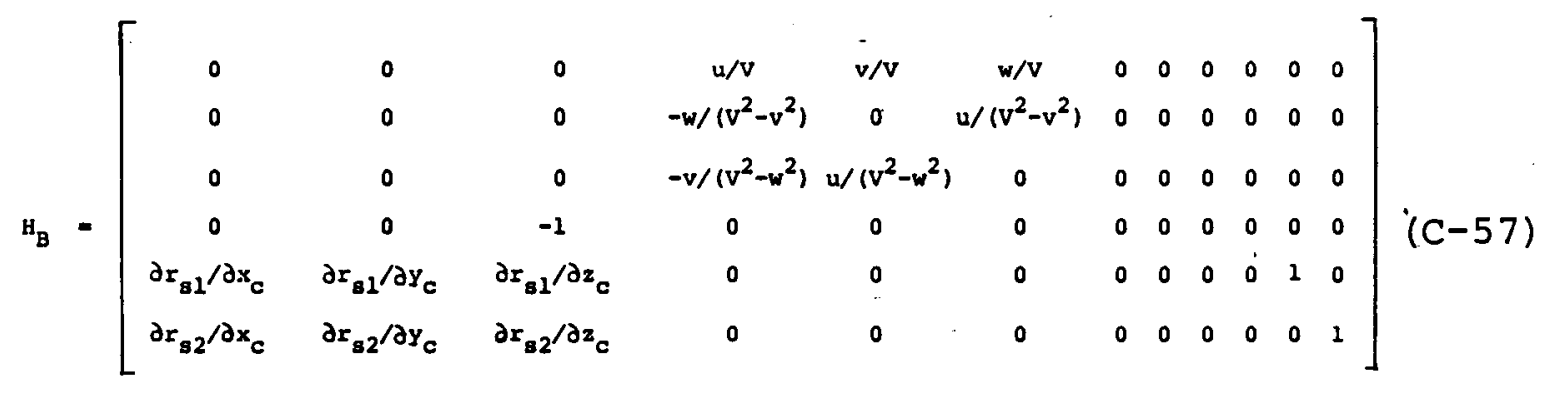

with

$$
\begin{aligned}
\partial r_{s i} / \partial x_{c}= & \left(r x l x_{s i}+r \times 2 y_{s i}+r x 3 z_{s i}\right) / r_{s i}(c-58) \\
\partial r_{s i} / \partial y_{c}= & (1-\gamma)\left[-\sin \left(\mu_{0}+\mu\right) x_{s i}+\cos \left(\mu_{o}+\mu\right) y_{s i}\right] / r_{s i}(c-59) \\
\partial r_{s i} / \partial z_{c}= & {\left[-\cos \left(\lambda_{0}+\lambda\right) \cos \left(\mu_{0}+\mu\right) x_{s i}-\cos \left(\lambda_{o}+\lambda\right)\right.} \\
& \left.\sin \left(\mu_{0}+\mu\right) y_{s i}-\sin \left(\lambda_{0}+\lambda\right) z_{s i}\right] / r_{s i} \quad(c-60)
\end{aligned}
$$

$\mathrm{x}_{\mathrm{si}}, \mathrm{y}_{\mathrm{si}}$ and $\mathrm{z}_{\mathrm{si}}$ are given in eq. (3-18). $\mathrm{rxl}, \mathrm{rx} 2$ and $\mathrm{rx3}$ are defined as follows,

$$
\begin{aligned}
& r \times 1=\varepsilon^{2} \sin 2\left(\lambda_{0}+\lambda\right) \cos \left(\lambda_{0}+\lambda\right) \cos \left(\mu_{0}+\mu\right)-(1-\gamma) \\
& {\left[\sin \left(\lambda_{0}+\lambda\right) \cos \left(\mu_{0}+\mu\right)+\tan \left(\lambda_{0}+\lambda\right) \sin \left(\mu_{0}+\mu\right) y_{c} / a\right](c-61)} \\
& r \times 2=\varepsilon^{2} \sin 2\left(\lambda_{0}+\lambda\right) \cos \left(\lambda_{0}+\lambda\right) \sin \left(\mu_{0}+\mu\right)-(1-\gamma) \\
& {\left[\sin \left(\lambda_{0}+\lambda\right) \sin \left(\mu_{0}+\mu\right)-\tan \left(\lambda_{0}+\lambda\right) \cos \left(\mu_{0}+\mu\right) y_{c} / a\right](c-62)} \\
& r \times 3=\varepsilon^{2} \sin 2\left(\lambda_{0}+\lambda\right) \sin \left(\lambda_{0}+\lambda\right) \quad+(1-\gamma) \\
& \cos \left(\lambda_{0}+\lambda\right)
\end{aligned}
$$




\section{3 IMPROVEMENT OF THE ACCURACY OF DME/DME=BASED POSITION} ESTIMATION VIA A DME/DME/DME SCHEME

The accuracy of the DME/DME navigational mode was discussed in Chapter 2. From equations (2-95) and (2-97),

$$
\sigma^{2}=\left(\sigma_{1}^{2}+\sigma_{2}^{2}\right) / s^{2} \theta=2 \sigma_{R}^{2} / \sigma^{2} \theta
$$

As shown in Chapter 3, assuming simultaneous engagement of three DME ground stations and zero biases and wind conditions, the position determination becomes a static estimation process. Under these circumstances the covariance formula, for comparison with eq. (c-64), is derived for the geometrical situation of Fig. C.l.

The aircraft is assumed to be at the coordinate origin in Fig. C.l ( $\left.x_{0}=0, y_{0}=0\right)$, navigational stations $A$ and $B$ will be used for reference (nonredundant) position estimation, and ground station c - for creation of the DME/DME/DME capability. Distance measurements to stations A and B are,

$$
\begin{aligned}
& r_{10}^{2}=\left(\hat{x}_{0}-x_{10}\right)^{2}+\left(\hat{y}_{0}-y_{10}\right)^{2} \\
& r_{20}^{2}=\left(\hat{x}_{0}-x_{20}\right)^{2}+\left(\hat{y}_{0}-y_{20}\right)^{2}
\end{aligned}
$$

Linearizing equations (C.-65) and (c-66) about the nominal trajectory and expressing the state estimation error 


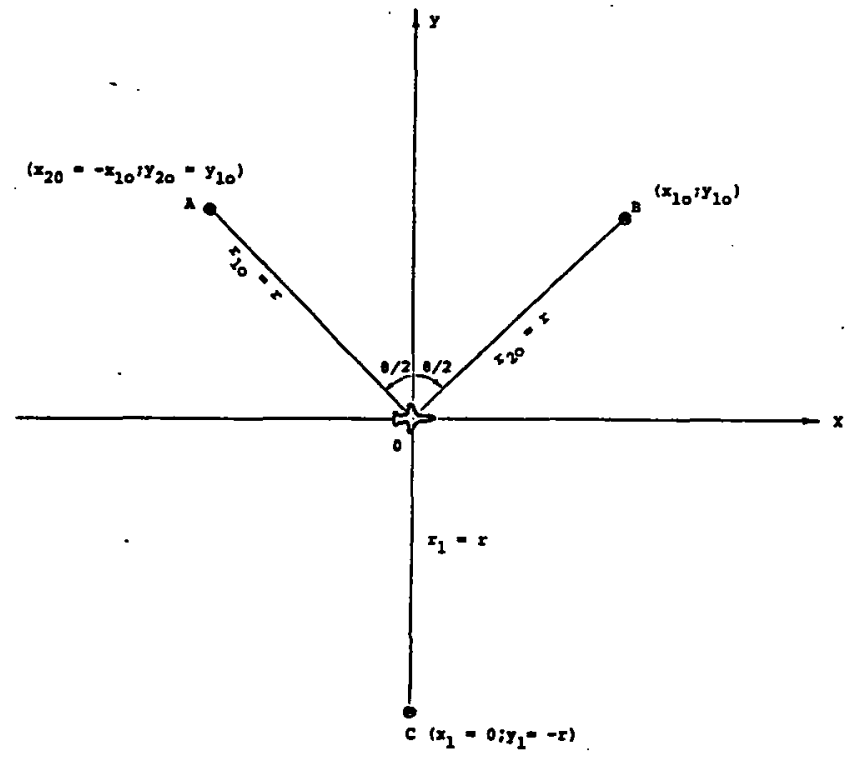

Figure C.1: Case Study of DME/DME/DME Position Estimation Accuracy

$\underline{x}_{0}=\left[\begin{array}{cc}\hat{x}_{0} & \hat{y}_{0}\end{array}\right]^{T}$ as a function of the measurement errors $\Delta r_{10}$ and $\Delta r_{20}$ '

$$
\left[\begin{array}{c}
\Delta \hat{x}_{0} \\
\Delta \hat{y}_{0}
\end{array}\right]=(r / 2 x y) \quad\left[\begin{array}{cc}
-y & y \\
-x & -x
\end{array}\right]\left[\begin{array}{c}
\Delta r_{10} \\
\Delta r_{20}
\end{array}\right]
$$

The covariance matrix for the nonredundant position estimation error vector $\Delta x_{0}$ is,

$$
P_{0}=E\left[{\underline{\Delta \hat{x}_{0}}}_{\underline{\Delta x}_{0}}\right]=\left(.25 x^{2} / x^{2} / y^{2}\right)\left[\begin{array}{cc}
-y & y \\
-x & -x
\end{array}\right]\left[\begin{array}{ll}
\sigma_{R 10}^{2} & \sigma_{R_{10}} \sigma_{R_{20}} \\
\sigma_{R 10} \sigma_{R 20} & \sigma_{R 20}^{2}
\end{array}\right]\left[\begin{array}{ll}
-y & y \\
& \\
-x & -x
\end{array}\right](C-68)
$$

$$
\text { c-10 }
$$


and assuming that the measurement errors to stations $A$ and $B$ are uncorrelated and possess equal statistics,

$$
\mathrm{P}_{\mathrm{O}}=\left(0.5 \mathrm{r}^{2} \sigma_{\mathrm{R}}^{2} / \mathrm{Y}^{2}\right)\left[\begin{array}{cc}
\mathrm{y}^{2} / \mathrm{x}^{2} & 0 \\
0 & 1
\end{array}\right]
$$

With some trigonometry based on Fig. C.I,

$$
\sigma_{\mathrm{O}}^{2}=\mathrm{P}_{\mathrm{Ol1}}+\mathrm{P}_{\mathrm{O} 22}=2 \sigma_{\mathrm{R}}^{2} / \mathrm{s}^{2} \theta
$$

So far this is another way of deriving the result of eq. (C-64). The advantage of this approach is that interpreting the nonredundant measurement case as the "system" (with no dynamics, i.e. $F=0$ and $\Phi=I$ ), the additional measurement may be accounted for in an optimal way. The distance measurement to station $C$ provides the measurement equation,

$$
\Delta z=\Delta r_{1}=r^{-1}\left[-x_{1}-y_{1}\right] \underline{\Delta x_{0}}+v_{1}=H_{1} \underline{\Delta x}_{0}+v_{1}
$$

The Kalman filter algorithm (discussed in Chapter 3) renders the state covariance matrix for this case,

$$
\mathrm{P}_{1}=\left[\mathrm{I}-\mathrm{K}_{1} \mathrm{H}_{1}\right] \mathrm{P}_{\mathrm{O}}
$$

where 


$$
K_{1}=P_{O} H_{1}^{T}\left[H_{1} P_{O} H_{1}^{T}+R_{1}\right]^{-1}
$$

with $\mathrm{R}_{1}=\sigma_{\mathrm{R}}^{2}$ of the third DME measurement. After completion of the algebraic substitutions,

$$
P_{1}=\left(0.5 r^{2} \sigma_{R}^{2} / y^{2}\right)\left[\begin{array}{cc}
y^{2} / x^{2} & 0 \\
0 & 1-(2+c \theta)^{-1}
\end{array}\right]
$$

and

$$
\sigma_{1}^{2}=\sigma_{0}^{2}-0.5{ }_{R}^{2} \cos ^{-2}(0.5 \theta) /\left[1+\cos ^{2}(0.5 \theta)\right]
$$

From equations $(c-70)$ and $(c-75)$,

$$
\left(\sigma_{1} / \sigma_{0}\right)^{2}=1-\sin ^{2}(0.5 \theta) /\left[1+2 \cos ^{2}(0.5 \theta)\right]
$$

For $\theta=90 \mathrm{deg}$, the improvement in position accuracy is 13.58, and for the ideal-GDOP-angle $(\theta=120 \mathrm{deg}$ for this case) it is 29.38 , with respect to the nonredundant case. 
Appendix D

POST-FLIGHT PROCESSING - DETAILS

\section{1 PREPROCESSING}

Post-flight data handling began using the HP-l000 digital computer located at Princeton University's Gas Dynamics Laboratory. The raw data was transferred to a 9-track, 1600 BPI magnetic tape that can be processed on either the IBM 4341 or the IBM 3081 computer. The block-diagram in Fig. D. 1 summarizes the described procedure.

The FORTRAN program CAT9 controled the transfer from the DCIOOA cartridges to the 9-track magnetic tape. The FORTRAN program RAWYl converted 16-bit binary-formatted data into IBM-compatible decimal integer format and arranged the data in physical time vectors. The FORTRAN program SPIFYl completed the preprocessing by converting the decimal integer time vectors into voltage and then - into engineering units, also converting Indicated Airspeed (IAS) to True AirSpeed (TAS). The listings of these software programs may be found in Ref. 17. 


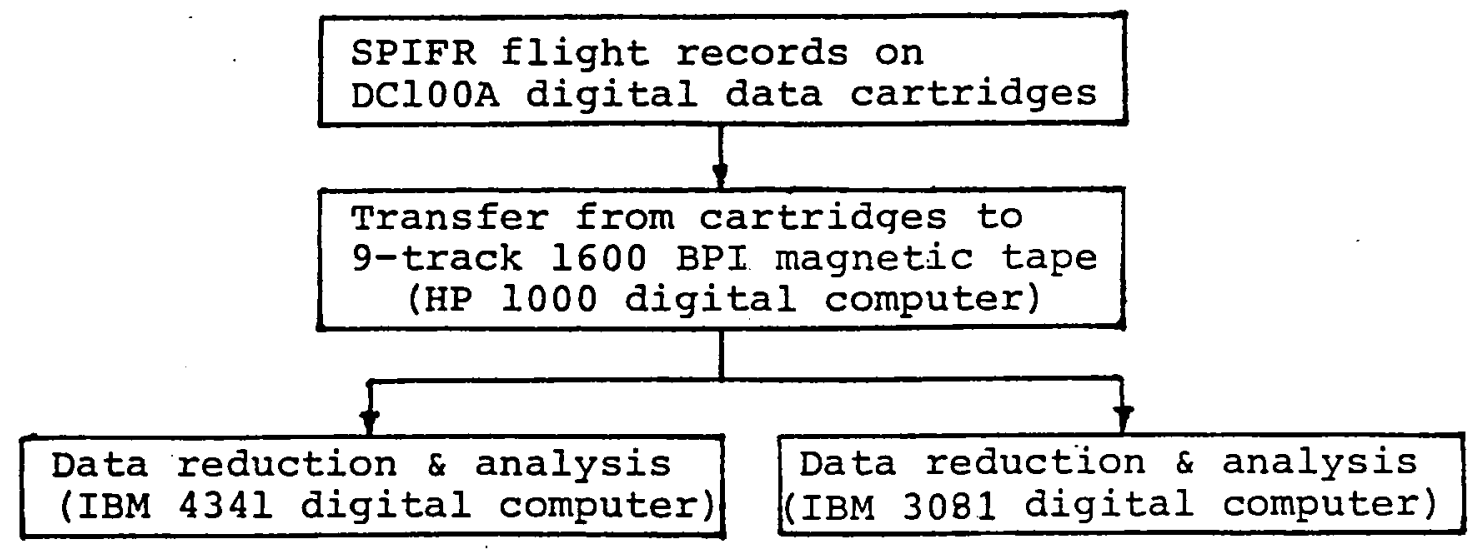

Figure D.1: Data Reduction Procedure

The SPIFR data storage policy was to preserve both the raw flight-test data and the preprocessed data on magnetic tapes (9-track, 1600 BPI), which made it compatible for further analysis on both the IBM 4341 and the IBM 3081 machines. Thus, two copies of the raw integer data (RAWYl output file) and one copy with engineering-unit-time-vectors (SPIFYl output) - for further processing (analysis, tabular printouts or plotting) were preserved. 


\section{2 OPTIMAL FLIGHT PATH RECONSTRUCTION $=$ INPUTS}

The optimal flight path reconstruction program package required several input parameters, which are specific to a given SPIFR test-flight:

* Record length of the data file to be processed.

* Time vectors of codes identifying the ground stations, the distances to which were recorded on the two DME channels. This information was extracted from printouts of the preprocessed data and in particular, was based on a channel dedicated to station switching timings. The code convention is given in Table D. 1 .

TABLE D. 1

Hexidecimal Code Recorded on the Channel that Provides Stations Switching Timings

\begin{tabular}{|c|c|c|c|c|}
\hline NAV2 NAVI & RBV & COL & GXU & SBJ \\
\hline RBV & EE & ED & EB & E7 \\
COL & DE & DD & DB & D7 \\
GXU & BE & BD & BB \\
SBJ & 7E & 7D & 78 \\
\hline
\end{tabular}


The stations are: RBV - Robbinsville, COL - Colts Neck, GXU - MCGuire and SBJ - Solberg. The stations' coordinates $\left(\lambda_{s i}, \mu_{s i}, z_{c s i}\right)$ were obtained from Ref. 73 (Table D.2). The information of Table D.2 is part of the flight path reconstruction program package, and for each test-flight two timing vectors were supplied as input, following the code convention: 1 - RBV, 2 - COL, 3 - GXU and 4 - SBJ.

* Approximate values for initial conditions of the state variables $\underline{x}_{O A}$ and $\underline{x}_{o B}$, based on printouts of preprocessed data.

* $Q_{W x}$ and $Q_{W Y}$, based on averaged before- and after-flight meteorological wind data and on Fig. 3.4.

The program package used the JCL EXEC Language to construct its input data files from the preprocessed data tape library. 
TABLE D. 2

Navigational Stations and Forrestal Airport Reference Point Coordinates

\begin{tabular}{|c|c|c|c|c|}
\hline $\begin{array}{l}\text { STATION } \\
\text { CODE }\end{array}$ & STATION & $N^{\lambda} 40^{\circ} x^{\prime}$ & $\|^{\mu}{ }^{\mu} 74^{\circ} Y^{\prime}$ & $\begin{array}{c}\mathrm{Z}_{\mathrm{I}} \\
(\mathrm{FT})\end{array}$ \\
\hline 1 & RBV & $12: 1$ & 29.7 & -250 \\
\hline 2 & $\mathrm{COL}$ & 18.7 & 09.6 & -120 \\
\hline 3 & GXU: & 00.6 & 35.8 & -120 \\
\hline 4 & SḄJ & $35.0^{\circ}$ & 44.5 & -190 \\
\hline $\begin{array}{c}--\cdots \\
0\end{array}$ & $\begin{array}{l}\text { FORRI: } \\
\text { Fons }\end{array}$ & 20.8 & $\begin{array}{l}\overline{-}-\overline{-} \\
36.6 .\end{array}$ & $\begin{array}{l}---\cdots \\
-109\end{array}$ \\
\hline
\end{tabular}

\section{3 OPTIMAL FLIGHT PATH RECONSTRUCTION $=$ PROGRAM FLOW CHARTS}

The program package flow charts are given in Figures D.2 and D.3. To avoid burdening this report, all computer codes, which were developed in the course of this research, were collected in a separate supplement, Ref. 17. This document is available upon request. 


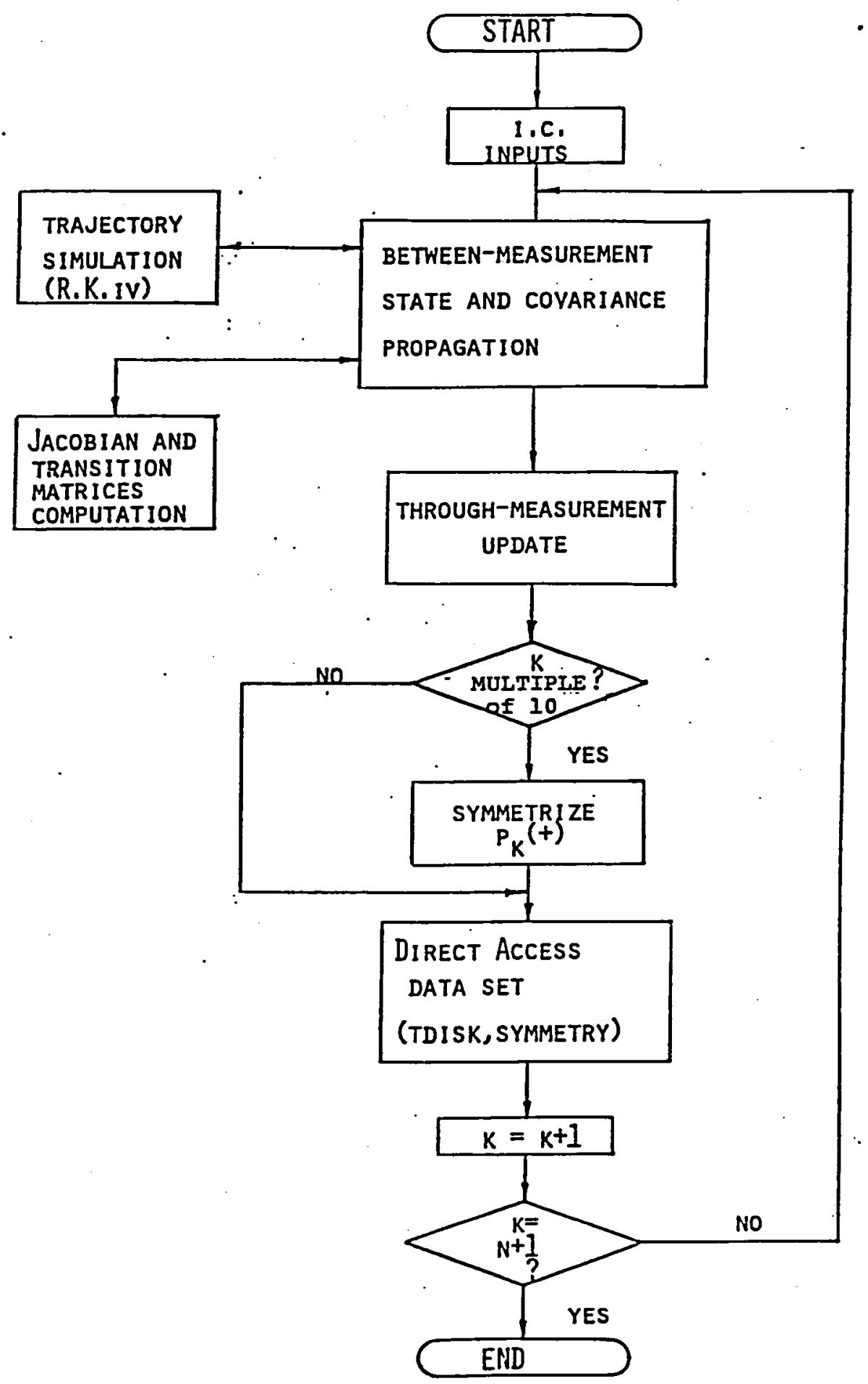

Figure D.2: Forward Filtering Program

D-6 


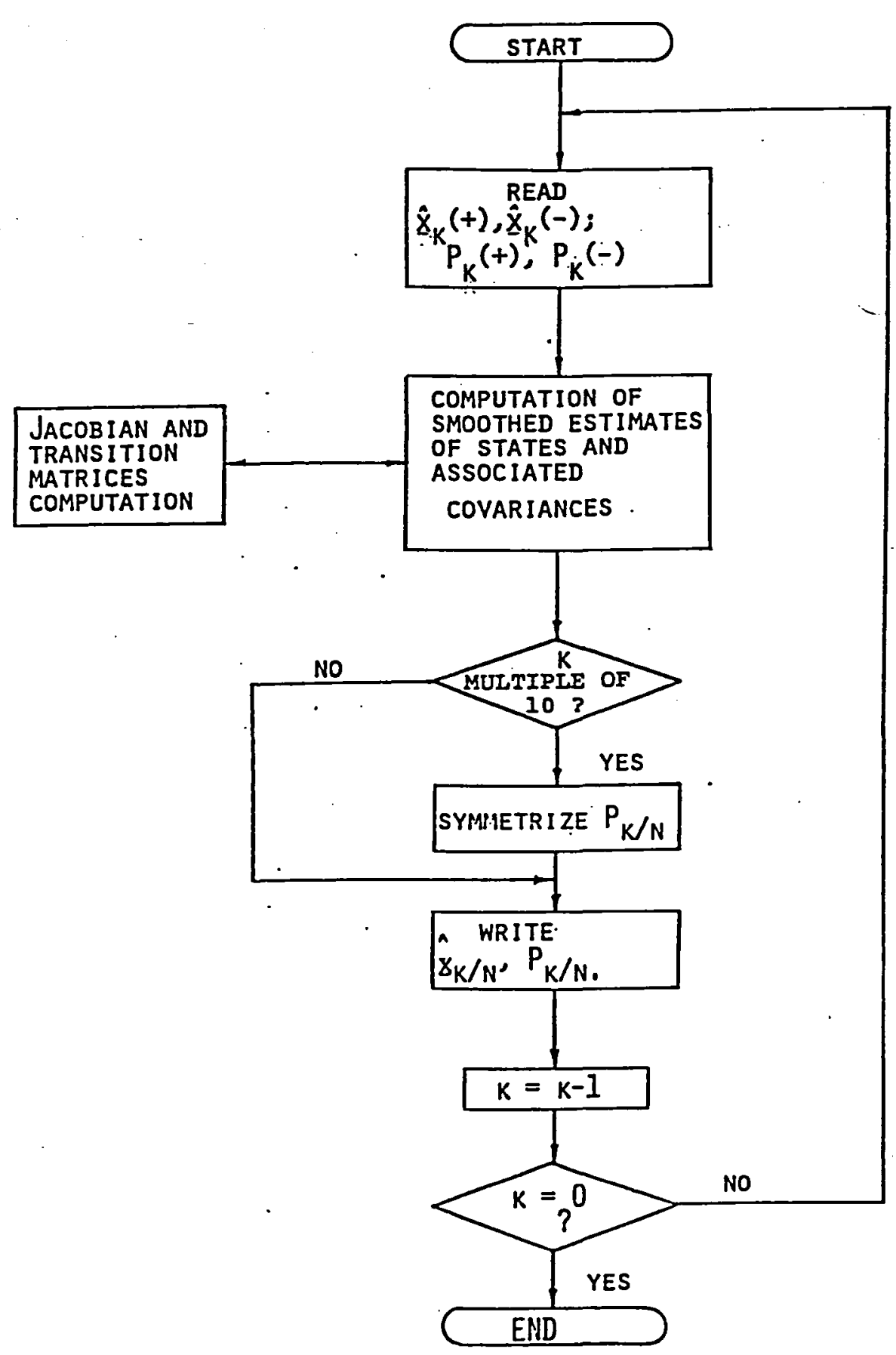

Figure D.3: Backward Smoothing Program 


\section{Appendix E \\ PILOT COMMENTS AND DATA SUMMARY}

Summaries of all the pilot comments and ratings are presented in this appendix for each configuration. All experimental results that are reported were obtained under zero-to-light turbulence conditions. As pointed out in Section 2.5, higher turbulence could have obscured the longperiod configuration trends. The wind data at 3000 ft (deg of azimuth/kt) was given by the regional flight weather service center. The numerical values are results of averaging before- and after-flight weather sequence data. For a better estimate of surface wind, information from Forrestal, Newark, and Trenton airfields, and from the McGuire Air Force Base was averaged for mid-takeoff missions (normally there were 3 missions per takeoff). The altimeter setting data corresponds to Forrestal airfield. As explained in Section 2.5, the navigational tracks, which were actually flown in the flight-test series, were No. 2, 3, and 4 (Fig. 2.5 to 2.7 ). Following the randomization principle (Chapter $2)$, the tracks were alternated, and were not repeated successively, unless flown on a different takeoff. The same principle was applied to the in-flight simulated aerodynamic configurations. Most of the configurations were flown 
twice. In some cases, when objective data recording problems occured due to checklist errors, a configuration was flown a third time. As detailed in Chapter 4, CHR is the Cooper-Harper Rating scale, and SSR is the Simpson-Sheridan workload scale. The experimental results reported here, were produced by the Princeton University Chief Test Pilot, whose flight experience amounts to over 600 logged IFR hours out of about 5000 total hours). His reference point, when referring to a configuration response as "Nominal", is the basic Navion configuration, which is characterized by eq. $(2-50)$ to $(2-67) \cdot{ }^{1}$

1 This basic configuration requires substantial (but essentially normal) power readjustment for airspeed retrimming to $105 \mathrm{kt}$. This biased upwards some speed retrimming PORS, and shows in the pilot's comments. 


\begin{tabular}{|c|c|c|c|}
\hline \multicolumn{4}{|c|}{ CONFIGURATION NO. $1 ;$ NOMINAL } \\
\hline \multicolumn{2}{|c|}{$\begin{array}{c}\text { Flight Test } \\
\text { No. }\end{array}$} & 1 & - \\
\hline \multicolumn{2}{|c|}{$\begin{array}{l}\text { Navigational } \\
\text { Track }\end{array}$} & 3 & 2 \\
\hline \multicolumn{2}{|c|}{$\begin{array}{l}\text { Wind at } 3000^{\prime} \\
{[\mathrm{deg} / \mathrm{kt}]}\end{array}$} & $340 / 14$ & $250 / 13$ \\
\hline \multicolumn{2}{|c|}{$\begin{array}{l}\text { Surface Wind } \\
{[\mathrm{deg} / \mathrm{kt}]}\end{array}$} & $330 / 5$ & $260 / 5$ \\
\hline \multicolumn{2}{|c|}{$\begin{array}{l}\text { Altimeter } \\
\text { Setting [in } \mathrm{Hg} \text { ] }\end{array}$} &.$\quad 30.16$ & 30.00 \\
\hline \multirow{3}{*}{ 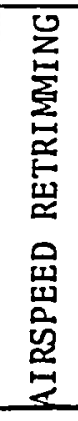 } & CHR & $3 \frac{1}{2}$ & 3 \\
\hline & SSR & $3 \frac{1}{2}$ & 3 \\
\hline & Comments & $\begin{array}{l}\text { A bit "sloppy" in getting } \\
\text { trimmed on new airspeed. }\end{array}$ & $\begin{array}{l}\text { Some pitch trim change } \\
\text { with speed change, but in } \\
\text { usual direction, ok. }\end{array}$ \\
\hline \multirow{3}{*}{ 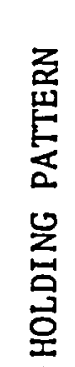 } & CHR & 3 & 3. \\
\hline & SSR & 3 & 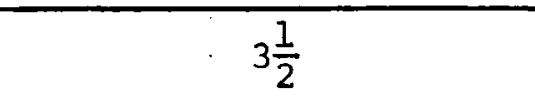 \\
\hline & Comments & Nominal. & Some adjustment for wind. \\
\hline \multirow{3}{*}{ 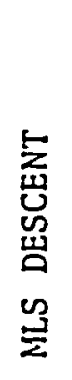 } & CHR & 3 & . \\
\hline & SSR & $3 \frac{1}{2}$ & 3 \\
\hline & Comments & $\begin{array}{l}\text { Some trouble with holding } \\
\text { glide slope needle at } \\
\text { zero. }\end{array}$ & No problem. \\
\hline \multirow[b]{3}{*}{ 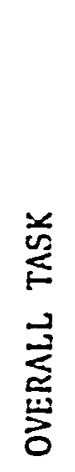 } & CHR & 3 & 3 \\
\hline & SSR & $3 \frac{1}{2}$ & 3 \\
\hline & Comments & Nominal task. & $\begin{array}{l}\text { Nominal with slight trim } \\
\text { workload. }\end{array}$ \\
\hline
\end{tabular}




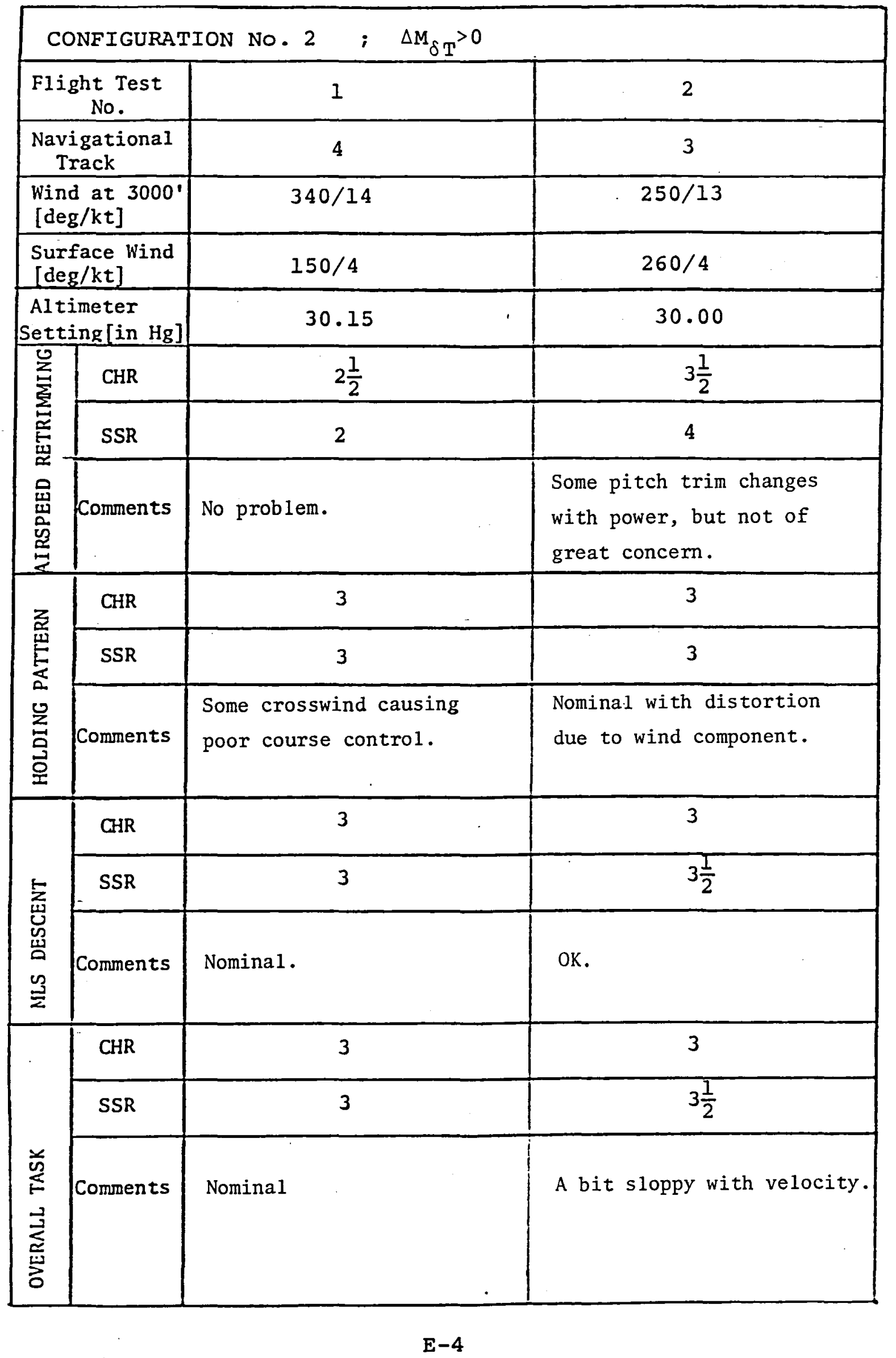




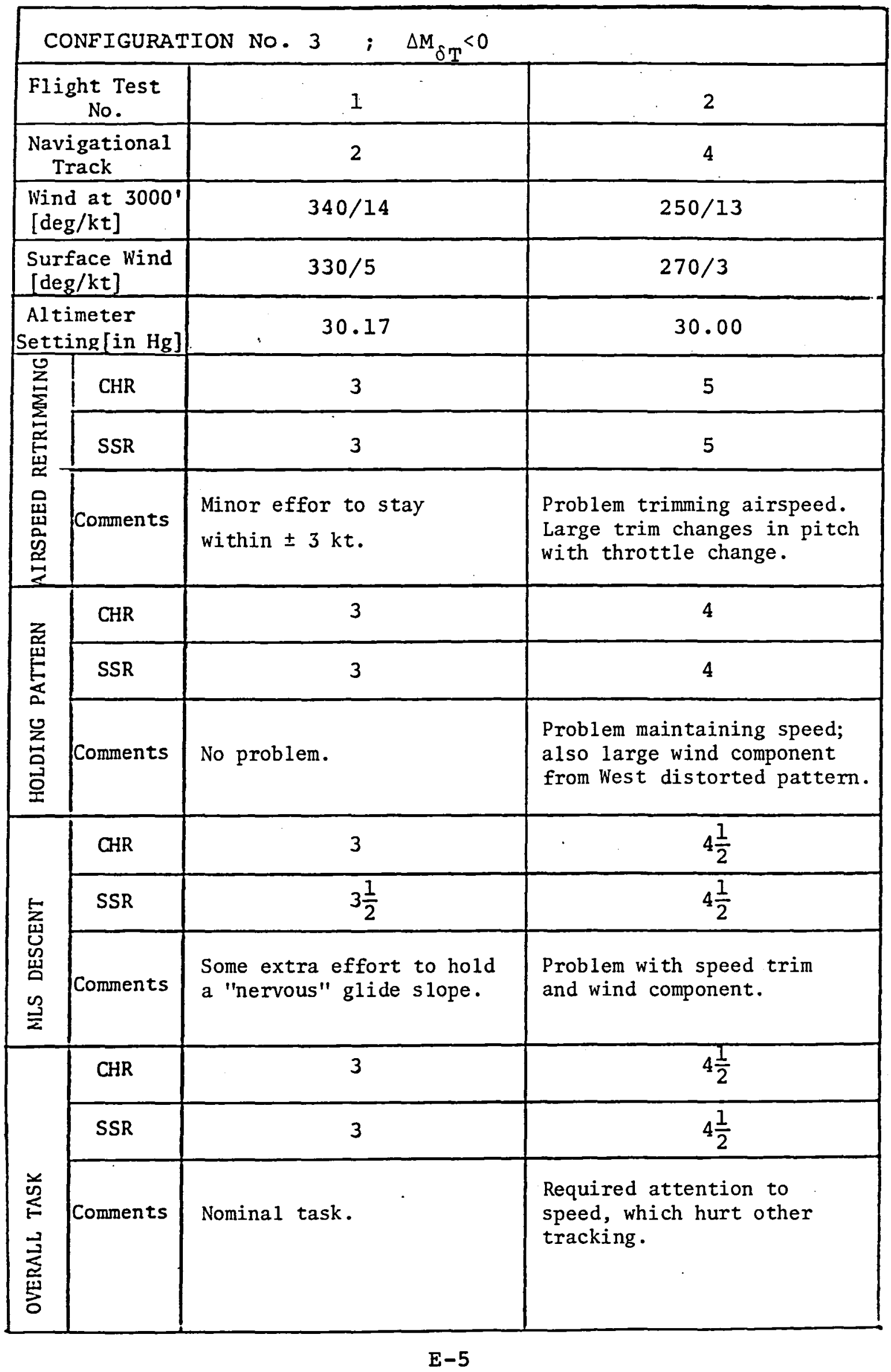




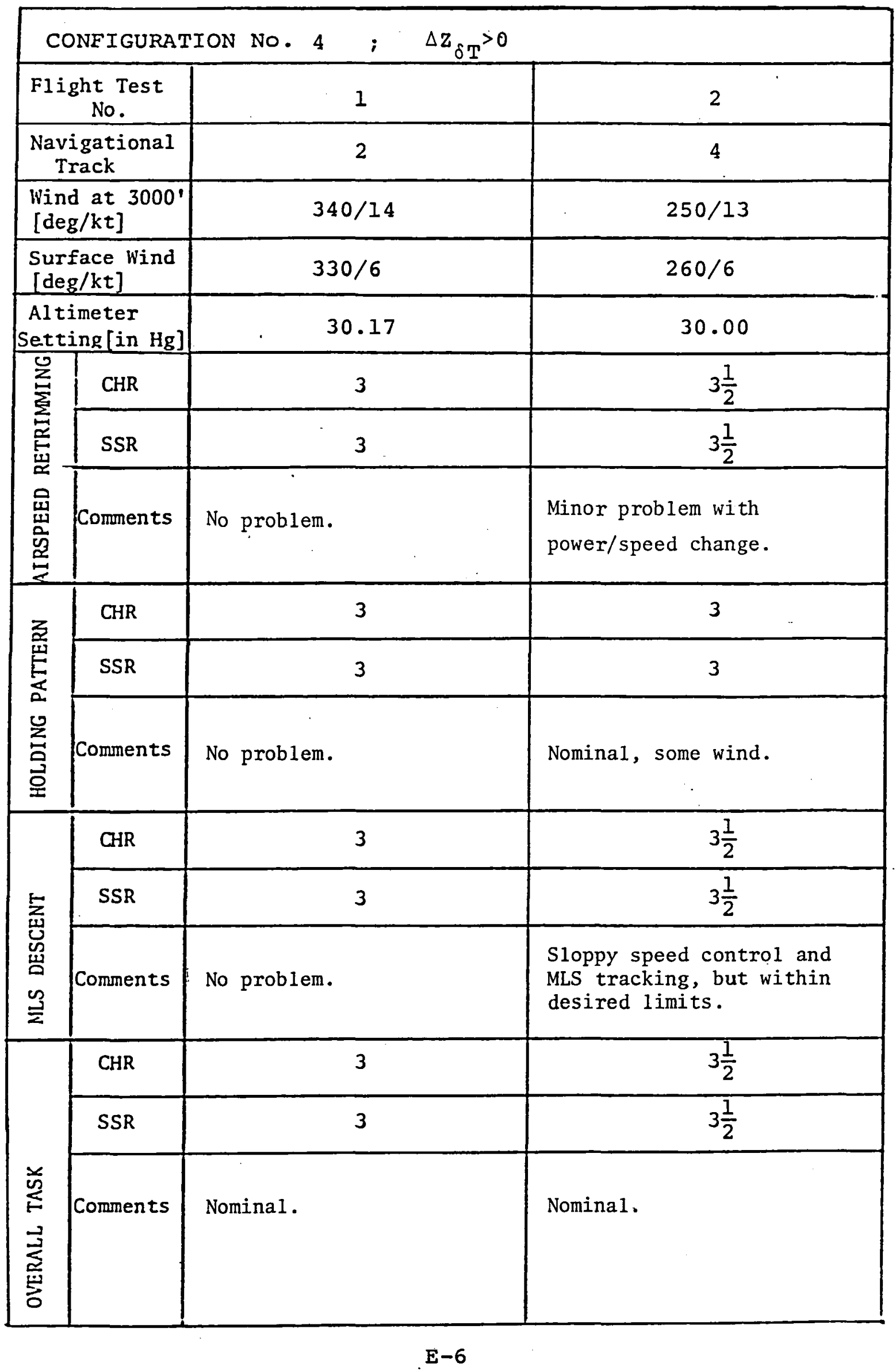




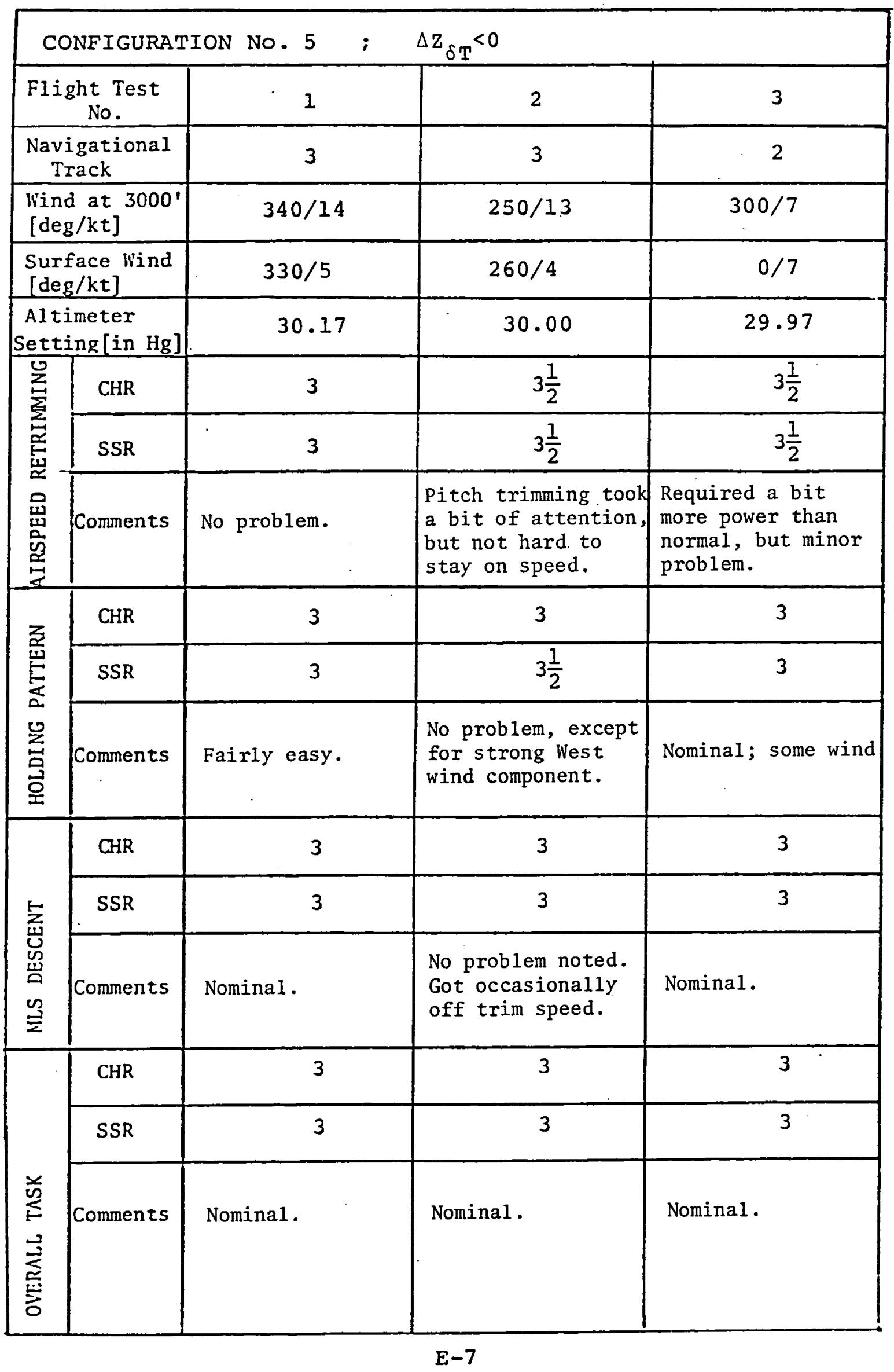




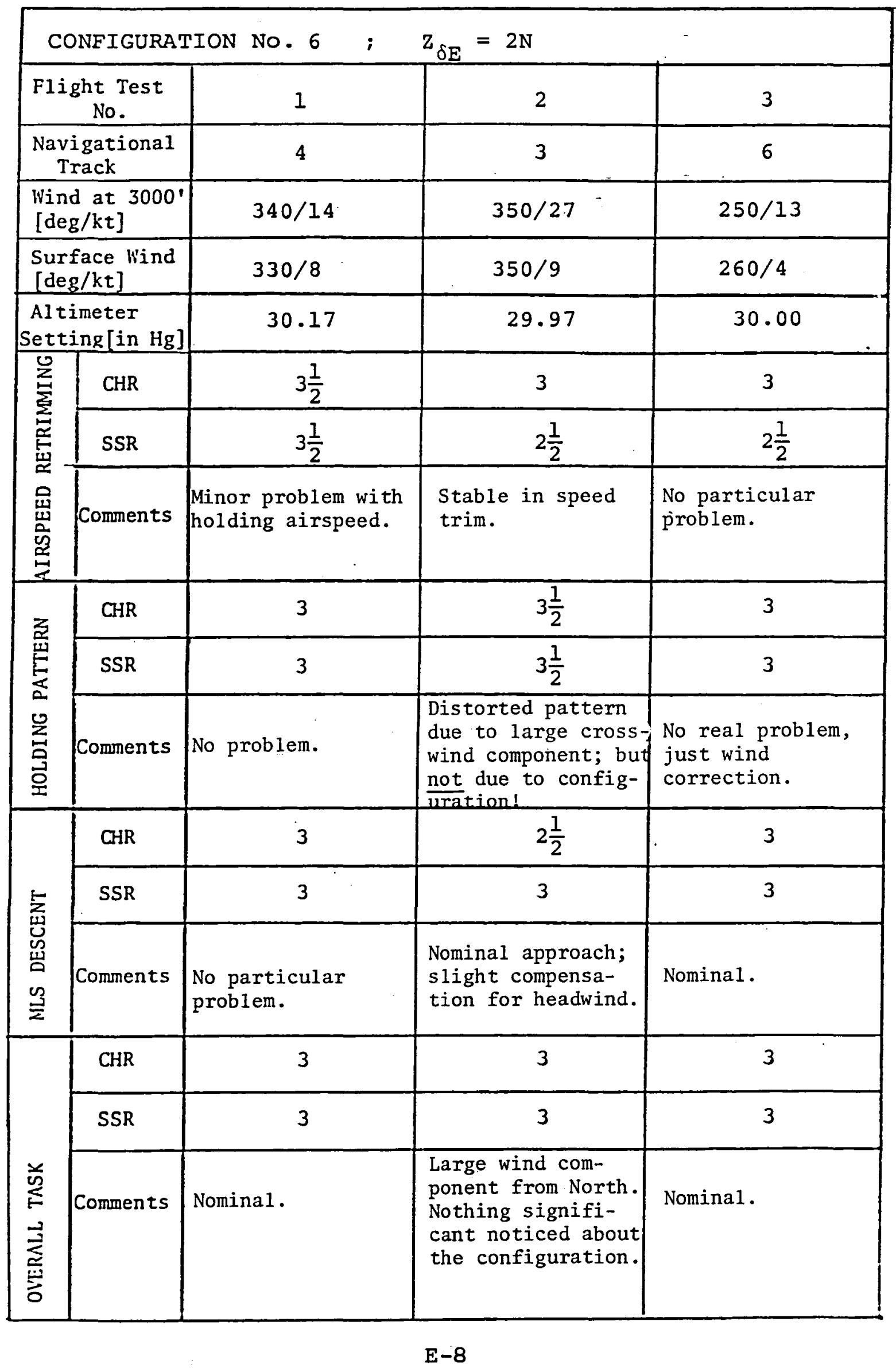




\begin{tabular}{|c|c|c|c|}
\hline \multicolumn{4}{|c|}{ CONEIGURATION NO. 7} \\
\hline \multicolumn{2}{|c|}{$\begin{array}{c}\text { Flight Test } \\
\text { No. }\end{array}$} & 1 & 2 \\
\hline \multicolumn{2}{|c|}{$\begin{array}{l}\text { Navigational } \\
\text { Track }\end{array}$} & 2 & 4 \\
\hline \multicolumn{2}{|c|}{$\begin{array}{l}\text { Wind at } 3000^{\prime} \\
{[\mathrm{deg} / \mathrm{kt}]}\end{array}$} & $350 / 11$ & $\mathrm{~L} / \mathrm{V}$ (light and variable) \\
\hline \multicolumn{2}{|c|}{$\begin{array}{l}\text { Surface Wind } \\
\text { [deg/kt] }\end{array}$} & $020 / 4$ & $90 / 9$ \\
\hline \multicolumn{2}{|c|}{$\begin{array}{l}\text { Altimeter } \\
\text { Setting [in } \mathrm{Hg}]\end{array}$} & 30.23 & 30.01 \\
\hline \multirow{2}{*}{ 寧 } & $\mathrm{CHR}$ & $2 \frac{1}{2}$ & 4 \\
\hline & SSR & $2 \frac{1}{2}$ & 4 \\
\hline 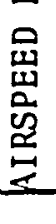 & Comments & No problem. & $\begin{array}{l}\text { A little "loose" in air- } \\
\text { speed holding. Requires } \\
\text { small corrections in } \\
\text { attitude. }\end{array}$ \\
\hline \multirow{3}{*}{ 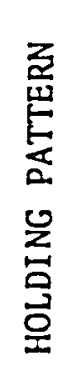 } & CHR & 3 & 3 \\
\hline & SSR & 3 & 3 \\
\hline & Comments & Fairly easy. & No particular problem. \\
\hline \multirow{3}{*}{ 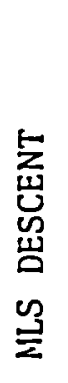 } & CHR & 3 & 3 \\
\hline & SSR & 3 & $3 \frac{1}{2}$ \\
\hline & Comments & Nominal & $\begin{array}{l}\text { Tracking OK; just required } \\
\text { power adjustments for } \\
\text { airspeed retrimming. }\end{array}$ \\
\hline \multirow[b]{3}{*}{ 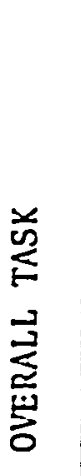 } & CHR & 3 & 3 \\
\hline & SSR & 3 & 3 \\
\hline & Comments & Nominal task. & $\begin{array}{l}\text { Nominal; just a little } \\
\text { airspeed concern. }\end{array}$ \\
\hline
\end{tabular}




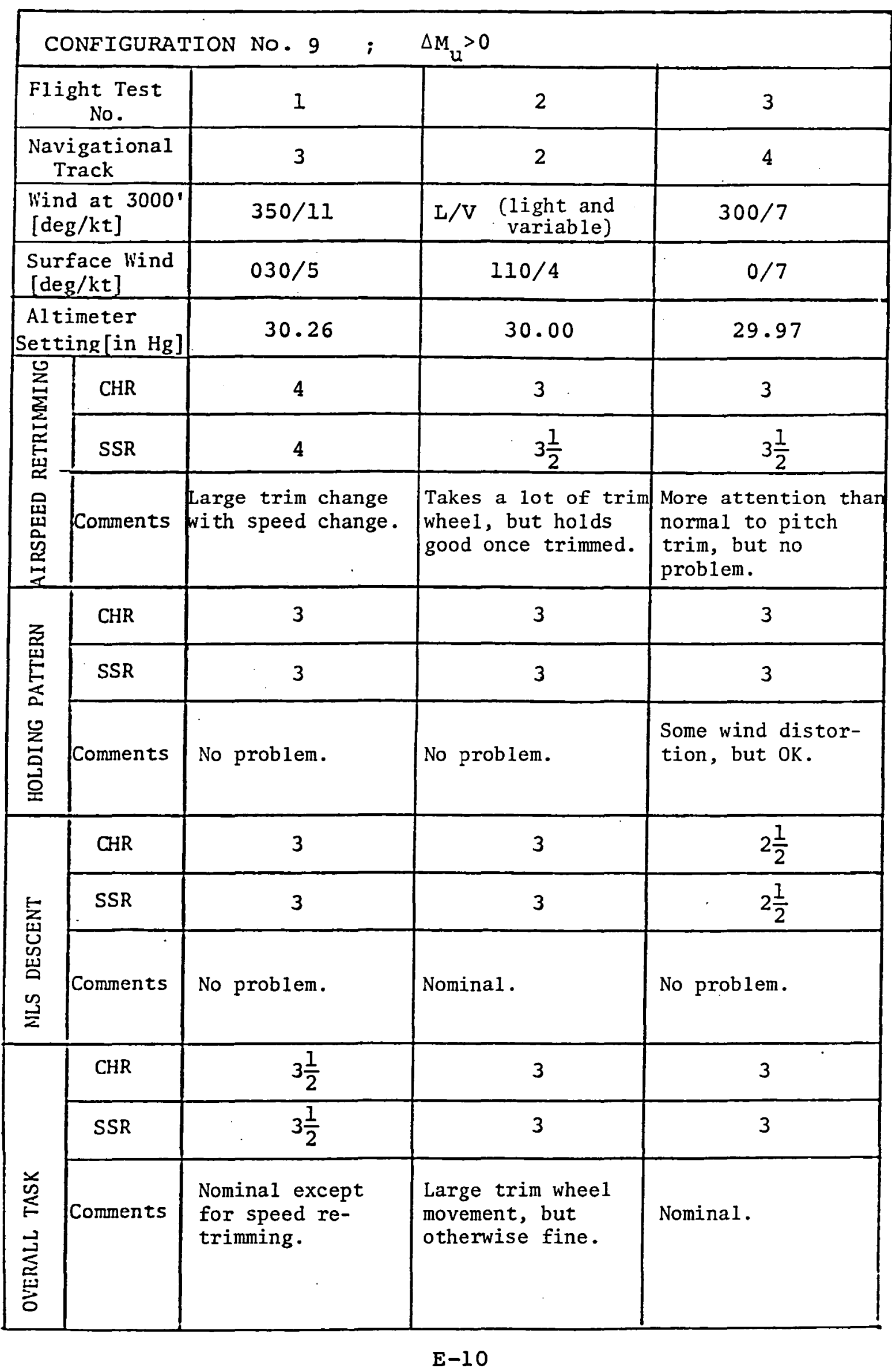




\begin{tabular}{|c|c|c|c|}
\hline \multicolumn{4}{|c|}{ CONFIGURATION NO. 10} \\
\hline \multicolumn{2}{|c|}{$\begin{array}{c}\text { Flight Test } \\
\text { No. }\end{array}$} & 1 & 2 \\
\hline \multicolumn{2}{|c|}{$\begin{array}{l}\text { Navigational } \\
\text { Track }\end{array}$} & 4 & 3 \\
\hline \multicolumn{2}{|c|}{$\begin{array}{l}\text { Wind at } 3000^{\prime} \\
{[\mathrm{deg} / \mathrm{kt}]}\end{array}$} & $350 / 11$ & $\mathrm{~L} / \mathrm{V}$ (light and variable) \\
\hline \multicolumn{2}{|c|}{$\begin{array}{l}\text { Surface Wind } \\
\text { [deg/kt] }\end{array}$} & $45 / 6$ & $100 / 7$ \\
\hline \multicolumn{2}{|c|}{$\begin{array}{l}\text { Altimeter } \\
\text { Setting [in } \mathrm{Hg}]\end{array}$} & 30.29 & 30.01 \\
\hline \multirow{3}{*}{ 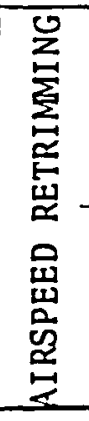 } & CHR & 4 & 5 \\
\hline & SSR & 4 & 5 \\
\hline & Comments & $\begin{array}{l}\text { Very "loose" in speed } \\
\text { control. No large forces, } \\
\text { actually low trim amounts } \\
\text { but constantly retrimming } \\
\text { to desired speed. }\end{array}$ & $\begin{array}{l}\text { Very unstable in speed trim; } \\
\text { constant attention and power } \\
\text { adjustments for attitude } \\
\text { trim. }\end{array}$ \\
\hline \multirow{3}{*}{ 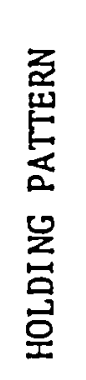 } & $\mathrm{CHR}$ & 3 & 3 \\
\hline & SSR & 3 & 3 \\
\hline & Comments & No particular problem. & $\begin{array}{l}\text { Nominal. Some loss of } \\
\text { tracking accuracy, while } \\
\text { copying clearances. }\end{array}$ \\
\hline \multirow{3}{*}{ 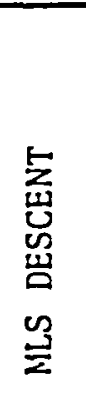 } & CHR & $3 \frac{1}{2}$ & 3 \\
\hline & SSR & 4 & 5 \\
\hline & Comments & $\begin{array}{l}\text { Additional effort of air- } \\
\text { speed holding while MLS } \\
\text { tracking. }\end{array}$ & $\begin{array}{l}\text { Able to track MLS quite } \\
\text { well, but constantly } \\
\text { chasing airspeed. }\end{array}$ \\
\hline \multirow[b]{3}{*}{ 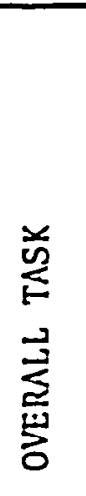 } & CHR & $3 \frac{1}{2}$ & $3 \frac{1}{2}$ \\
\hline & SSR & 4 & $4 \frac{1}{2}$ \\
\hline & Comments & $\begin{array}{l}\text { Moderate effort in speed } \\
\text { holding. }\end{array}$ & $\begin{array}{l}\text { Airspeed variations up to } \\
\pm 8 \mathrm{kt} \text { are annoying, but do } \\
\text { not degrade the task } \\
\text { appreciably. }\end{array}$ \\
\hline
\end{tabular}




\begin{tabular}{|c|c|c|c|}
\hline \multicolumn{4}{|c|}{ CONFIGURATION No. 11} \\
\hline \multicolumn{2}{|c|}{$\begin{array}{c}\text { Flight Test } \\
\text { No. } \\
\end{array}$} & 1 & 2 \\
\hline \multicolumn{2}{|c|}{$\begin{array}{l}\text { Navigational } \\
\text { Track }\end{array}$} & 3 & 2 \\
\hline \multicolumn{2}{|c|}{$\begin{array}{l}\text { Wind at } 3000^{\prime} \\
{[\mathrm{deg} / \mathrm{kt}]}\end{array}$} & L/V (light and variable) & $300 / 7$ \\
\hline \multicolumn{2}{|c|}{$\begin{array}{l}\text { Surface Wind } \\
\text { [deg/kt] }\end{array}$} & $110 / 4$ & $290 / 5$ \\
\hline \multicolumn{2}{|c|}{$\begin{array}{l}\text { Altimeter } \\
\text { Setting [in } \mathrm{Hg}] \\
\end{array}$} & 30.00 & 29.95 \\
\hline \multirow{3}{*}{ 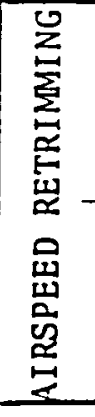 } & $\mathrm{CHR}$ & 4 & $2 \frac{1}{2}$ \\
\hline & SSR & 4 & 3 \\
\hline & Comments & $\begin{array}{l}\text { Can change airspeed fairly } \\
\text { rapidly, but seems a bit } \\
\text { hard to hold; takes } \\
\text { constant attention. }\end{array}$ & No particular problem. \\
\hline \multirow{3}{*}{ 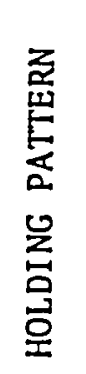 } & CHR & 3 & 3 \\
\hline & SSR & 3 & 3 \\
\hline & Comments & $\begin{array}{l}\text { No particular problem, } \\
\text { just monitoring speed and } \\
\text { taking clearances. }\end{array}$ & $\begin{array}{l}\text { Some North wind component } \\
\text { detected. }\end{array}$ \\
\hline \multirow{3}{*}{ 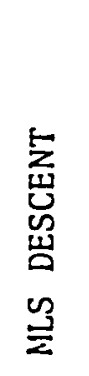 } & CHR & 3 & 3 \\
\hline & SSR & 4 & 3 \\
\hline & Comments & $\begin{array}{l}\text { Tracking } O K, \text { just busy } \\
\text { with speed trimming } \\
\text { with power. }\end{array}$ & Nominal. \\
\hline \multirow[b]{3}{*}{$\begin{array}{l}\frac{u}{5} \\
\vdots \\
\vdots \\
\vdots \\
\vdots \\
\vdots\end{array}$} & CHR & 3 & 3 \\
\hline & SSR & 4 & 3 \\
\hline & Comments & $\begin{array}{l}\text { Speed holding a little } \\
\text { sloppy. }\end{array}$ & No problem. \\
\hline
\end{tabular}




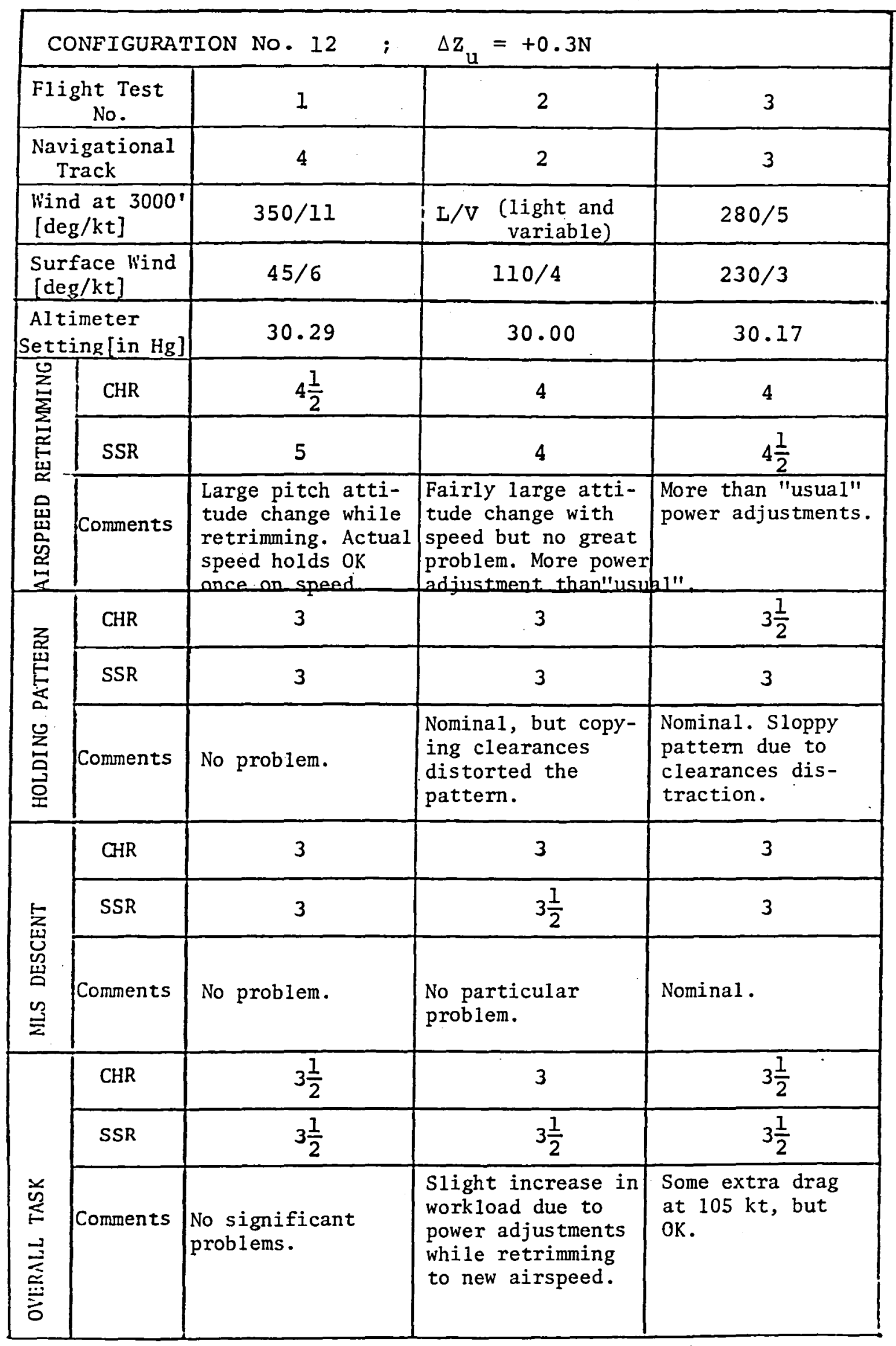




\begin{tabular}{|c|c|c|c|}
\hline \multicolumn{4}{|c|}{ CONFIGURATION No. 13} \\
\hline \multicolumn{2}{|c|}{$\begin{array}{c}\text { Flight Test } \\
\text { No. }\end{array}$} & 1 & 2 \\
\hline \multicolumn{2}{|c|}{$\begin{array}{l}\text { Navigational } \\
\text { Track }\end{array}$} & 4 & 3 \\
\hline \multicolumn{2}{|c|}{$\begin{array}{l}\text { Wind at } 3000^{\circ} \\
{[\mathrm{deg} / \mathrm{kt}]}\end{array}$} & $\mathrm{L} / \mathrm{V}($ light and variable) & $300 / 7$ \\
\hline \multicolumn{2}{|c|}{$\begin{array}{l}\text { Surface Wind } \\
{[\mathrm{deg} / \mathrm{kt}]}\end{array}$} & $110 / 4$ & $010 / 6$ \\
\hline \multicolumn{2}{|c|}{$\begin{array}{l}\text { Altimeter } \\
\text { Setting [in } \mathrm{Hg}]\end{array}$} & 30.00 & 29.97 \\
\hline \multirow{3}{*}{ 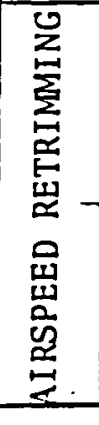 } & CHR & 5 & 3 \\
\hline & SSR & 5 & 4 \\
\hline & Comments & $\begin{array}{l}\text { Airspeed wanders and } \\
\text { requires constant attention } \\
\text { to power setting. }\end{array}$ & $\begin{array}{l}\text { Noted rapid deceleration } \\
\text { in speed reduction. }\end{array}$ \\
\hline \multirow{3}{*}{ 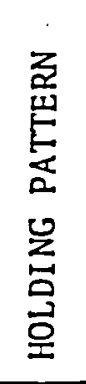 } & CHR & 3 & 3 \\
\hline & SSR & 3 & 3 \\
\hline & Comments & $\begin{array}{l}\text { Slight concern with small } \\
\text { wind component. }\end{array}$ & Adjustments for wind. \\
\hline \multirow{3}{*}{ 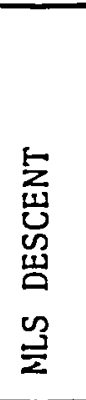 } & CHR & 3 & $2 \frac{1}{2}$ \\
\hline & SSR & 4 & 3 \\
\hline & Comments & $\begin{array}{l}\text { A bit of a bother } \\
\text { holding airspeed. }\end{array}$ & OK. \\
\hline \multirow[b]{3}{*}{ 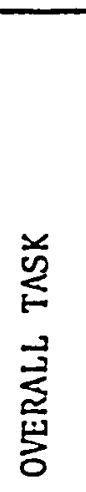 } & CHR & $3 \frac{1}{3}$ & 3 \\
\hline & SSR & 4 & 3 \\
\hline & Comments & "Sloppy" airspeed control. & $\begin{array}{l}\text { Some monitoring of power } \\
\text { required. }\end{array}$ \\
\hline
\end{tabular}




\begin{tabular}{|c|c|c|c|}
\hline \multicolumn{3}{|c|}{ CONFIGURATION NO. 14} & \\
\hline \multicolumn{2}{|c|}{$\begin{array}{c}\text { Flight Test } \\
\text { No. }\end{array}$} & 1 & 2 \\
\hline \multicolumn{2}{|c|}{$\begin{array}{l}\text { Navigational } \\
\text { Track }\end{array}$} & 3 & 3 \\
\hline \multicolumn{2}{|c|}{$\begin{array}{l}\text { Wind at } 3000^{\prime} \\
\text { [deg/kt] }\end{array}$} & $300 / 7$ & $300 / 7$ \\
\hline \multicolumn{2}{|c|}{$\begin{array}{l}\text { Surface Wind } \\
\text { [deg/kt] }\end{array}$} & $230 / 5$ & $010 / 6$ \\
\hline \multicolumn{2}{|c|}{$\begin{array}{l}\text { Altimeter } \\
\text { Setting [in } \mathrm{Hg}]\end{array}$} & 30.22 & 29.97 \\
\hline \multirow{2}{*}{ 突 } & CHR & 4 & 3 \\
\hline & SSR & $4 \frac{1}{2}$ & $3 \frac{1}{2}$ \\
\hline 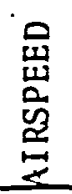 & Comments & $\begin{array}{l}\text { Needed several readjust- } \\
\text { ments of power to trim } \\
\text { to new airspeed. }\end{array}$ & $\begin{array}{l}\text { Noted slow airspeed change, } \\
\text { took longer to trim and } \\
\text { adjust throttle but minor } \\
\text { problem. }\end{array}$ \\
\hline \multirow{3}{*}{ 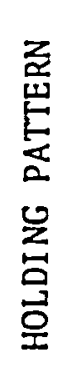 } & CHR & 3 & 3 \\
\hline & SSR & $3 \frac{1}{2}$ & 3 \\
\hline & Comments & $\begin{array}{l}\text { Strong wind component re- } \\
\text { quires some planning to } \\
\text { keep pattern at proper size }\end{array}$ & Some distortion due to wind. \\
\hline \multirow{3}{*}{ 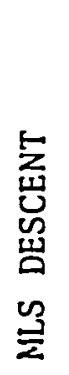 } & CHR & 3 & 3 \\
\hline & SSR & 3 & 3 \\
\hline & Comments & Nominal. & OK. \\
\hline \multirow[b]{3}{*}{ 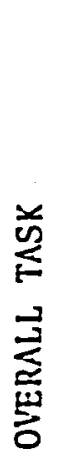 } & CHR & 3 & 3 \\
\hline & SSR & $3 \frac{1}{2}$ & 3 \\
\hline & Comments & Nominal. & $\begin{array}{l}\text { Nominal, with some work } \\
\text { with throttle adjustments. }\end{array}$ \\
\hline
\end{tabular}




\begin{tabular}{|c|c|c|c|}
\hline \multicolumn{2}{|c|}{ CONFIGURATION NO. 15} & \multicolumn{2}{|c|}{$\Delta \mathrm{Z}_{\mathrm{W}}=-0.5 \mathrm{~N}$} \\
\hline \multicolumn{2}{|c|}{$\begin{array}{c}\text { Flight Test } \\
\text { No. } \\
\end{array}$} & 1 & 2 \\
\hline \multicolumn{2}{|c|}{$\begin{array}{l}\text { Navigational } \\
\text { Track }\end{array}$} & 2 & 3 \\
\hline \multicolumn{2}{|c|}{$\begin{array}{l}\text { Wind at } 3000^{\prime} \\
{[\mathrm{deg} / \mathrm{kt}]}\end{array}$} & $300 / 7$ & $300 / 7$ \\
\hline \multicolumn{2}{|c|}{$\begin{array}{l}\text { Surface Wind } \\
{[\mathrm{deg} / \mathrm{kt}]}\end{array}$} & $250 / 6$ & $250 / 6$ \\
\hline \multicolumn{2}{|c|}{$\begin{array}{l}\text { Altimeter } \\
\text { Setting [in } \mathrm{Hg}]\end{array}$} & 30.20 & 30.20 \\
\hline \multirow{3}{*}{ 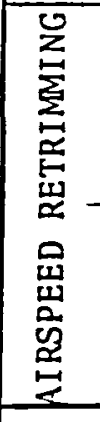 } & CHR & 4 & 5 \\
\hline & SSR & 4 & 5 \\
\hline & Comments & $\begin{array}{l}\text { Large pitch attitude } \\
\text { change with speed change. }\end{array}$ & $\begin{array}{l}\text { Large attitude excursions } \\
\text { and trim wheel motions. }\end{array}$ \\
\hline \multirow{3}{*}{ 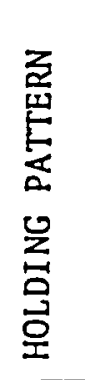 } & $\mathrm{CHR}$ & 3 & 4 \\
\hline & SSR & $3 \frac{1}{2}$ & 4 \\
\hline & Comments & $\begin{array}{l}\text { Mild compensation for } \\
\text { strong wind components. }\end{array}$ & $\begin{array}{l}\text { Distracting problems with } \\
\text { attitude and speed re- } \\
\text { trimming. }\end{array}$ \\
\hline \multirow{3}{*}{ 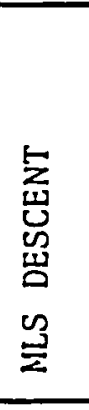 } & CHR & $3 \frac{1}{2}$ & 4 \\
\hline & SSR & 4 & 5 \\
\hline & Comments & $\begin{array}{l}\text { Requires a little extra } \\
\text { effort to get trimmed on } \\
\text { airspeed and at correspond- } \\
\text { ing attitude. }\end{array}$ & $\begin{array}{l}\text { Distracting problem with } \\
\text { attitude retrimming. }\end{array}$ \\
\hline \multirow[b]{3}{*}{ 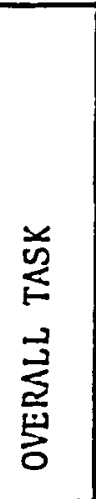 } & $\mathrm{CHR}$ & $3 \frac{1}{2}$ & $4 \frac{1}{2}$ \\
\hline & SSR & 4 & 5 \\
\hline & Comments & $\begin{array}{l}\text { A little extra concern due } \\
\text { to "different" relation- } \\
\text { ships between parameters. }\end{array}$ & $\begin{array}{l}\text { Large attitude and trim } \\
\text { changes. }\end{array}$ \\
\hline
\end{tabular}

$$
\text { E-16 }
$$




\begin{tabular}{|c|c|c|c|}
\hline \multicolumn{4}{|c|}{ CONEIGURATION NO. 16} \\
\hline \multicolumn{2}{|c|}{$\begin{array}{c}\text { Flight Test } \\
\text { No. }\end{array}$} & 1 & 2 \\
\hline \multicolumn{2}{|c|}{$\begin{array}{l}\text { Navigational } \\
\text { Track }\end{array}$} & 4 & 4 \\
\hline \multicolumn{2}{|c|}{$\begin{array}{l}\text { Wind at } 3000^{\prime} \\
{[\mathrm{deg} / \mathrm{kt}]}\end{array}$} & $300 / 7$ & $300 / 7$ \\
\hline \multicolumn{2}{|c|}{$\begin{array}{l}\text { Surface Wind } \\
{[\mathrm{deg} / \mathrm{kt}]}\end{array}$} & $240 / 5$ & $330 / 5$ \\
\hline \multicolumn{2}{|c|}{\begin{tabular}{|l|} 
Altimeter \\
Setting [in $\mathrm{Hg}]$
\end{tabular}} & 30.21 & 29.97 \\
\hline \multirow{3}{*}{ 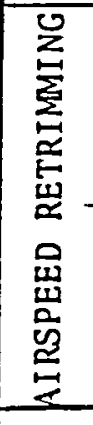 } & CHR & $2 \frac{1}{2}$ & 3 \\
\hline & SSR & 2 & 3 \\
\hline & Comments & No problem. & No significant problem. \\
\hline \multirow{3}{*}{ 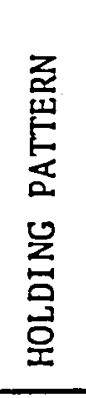 } & $\mathrm{CHR}$ & 3 & 3 \\
\hline & SSR & $3 \frac{1}{2}$ & 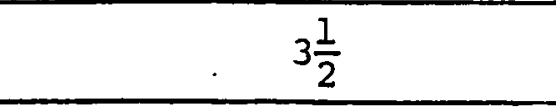 \\
\hline & Comments & $\begin{array}{l}\text { Strong wind required a } \\
\text { little compensation to } \\
\text { minimize pattern distortion. }\end{array}$ & $\begin{array}{l}\text { Somewhat distorted pattern } \\
\text { due to NE wind of } \\
\text { significance. }\end{array}$ \\
\hline \multirow{3}{*}{ 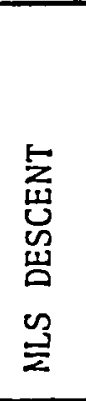 } & CHR & 3 & $2 \frac{1}{2}$ \\
\hline & SSR & 3 & 3 \\
\hline & Comments & No problem. & OK. \\
\hline \multirow[b]{3}{*}{ 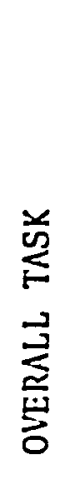 } & CHR & 3 & 3 \\
\hline & SSR & 3 & 3 \\
\hline & Comments & Nominal. & Nomina1. \\
\hline
\end{tabular}




\section{REFERENCES}

1. Anon.,"1977 Statistical Data", General Aviation Manufacturers Association, Washington, 1977.

2. Parkinson, R.C., "A Fuel-Efficient Cruise Performance Model for General Aviation Piston Engine Airplanes", $\mathrm{Ph} . \mathrm{D}$. Thesis, Department of Mechanical and Aerospace Engineering, Princeton University, June 1982.

3. Forsyth, D.L.; and Shaughnessy, J.D., "Single Pilot IFR Operating Problems Determined from Accident Data Analysis", NASA TM 78773, September 1979.

4. Loschke, P.C., et al., "Handling Qualities of Light Aircraft with Advanced Control Systems and Displays", in NASA Aircraft Safety and Operating Probiems, Vol. 1, NASA SP-270, MaY 1971.

5. Federal Aviation Regulations, Part 23, "Airworthiness Standards: Normal, Utility and Aerobatic Category Airplanes", Department of Transportation, Federal Aviation Administration, December 1969.

6. Breuhaus,W.O., "Resume of Flight Experience with the Phugoid Characteristics of Aircraft and Possible Methods for Improving These Characteristics on Future Aircraft", Cornell Aeronautical Laboratory FRM No. 117, May 1951.

7. Heilenday, F.W., "Artificial Stability and Control of Longitudinal Motion of the B-26 Aircraft", Cornell Aeronautical Laboratory, TB-757-F-2, November 1951 .

8. Newell, F., and Campbell, G., "Flight Evaluation of Variable ShortPeriod and Phugoid Characteristics in a B-26", WADC TR 54-594, 1954.

9. Anon., "Flying Qualities of Piloted Airplanes", Military Specification MIL-F-8785C, November 1980.

10. Ellis, D.R., "Flying Qualities of Small General Aviation Airplanes. Part 4. Review of Recent In-Flight Simulation Experiments and Some Suggested Criteria", FAA-RD-71-118, December 1971 . 
11. Ellis, D.R., "Flying Qualities of Small General Aviation Airplanes. Part 3. The Influence of Short Period Frequency and Damping, Pitch Control

Sensitivity, and Lift Curve Slope", FAA-RD-71-4, December 1971.

12. Ellis, D.R., and Griffith, C.L., "A Study of Longitudinal Controllability and Stability Requirements for Small General Aviation Airplanes", FAA-RD-78-113, August 1978.

13. Hinton, D.A., and Shaughnessy, J.D., "Adaptation of Time Line Analysis Program to single Pilot Instrument Flight Research", NASA TM 78748, August 1978.

14. Bergeron, H.P., "General Aviation Single Pilot IFR Autopilot Study", in NASA CP2170, November 1980.

15. Pennington, J.E., "Single Pilot Scanning Behaviour in Simulated Instrument Flight", NASA TM 80178, October 1979.

16. Adams, J.J., "Simulator Study of Conventional General Aviation Instrument Displays in Path Following Tasks", NASA TP 1776, December 1980.

17. Bar-Gill, A., "Supplement to 'Longitudinal Flying Qualities Criteria for Single Pilot Instrument Flight Operations', MAE-1576-T", MAE-1577, Princeton

University, October 1982.

18. Stengel, R.F., and Nixon, W.B., "Investigation of the Stalling Characteristics of a General Aviation Aircraft", Journal of Aircraft, Vol. 19, No. 6, June 1982 .

19. Bryson, A.E., and Denham, W.T., "A Steepest-Acsent Method for Solving Optimum Programming Problems", Journal of Applied Mechanics, Transactions of the ASME, Vol. 29, Series E No. 2, June 1962, pp. 247-257.

20. Shivers, J.P., "Full-Scale Wind-Tunnel Investigation of the Static Longitudinal and Iateral Characteristics of a Light Single-Engine Low-Wing Airplane", NASA TN D-5857, June 1970 .

21. Etkin, B., Dynamics of Atmospheric Flight, J. Wiley and Sons, New York, 1972.

22. Brogan, W.I., Modern Control Theory, Quantum Publishers Inc., New York, 1974.

23. Hildebrand, F.B., Introduction to Numerical Analysis, McGraw-Hill, New York, 1974. 
24. Stengel, R.F., "Equilibrium Response of Flight Control Systems", Automatica, Vol. 18, No. 3, 1982 .

25. Seckel, E., Stability and Control of Airplanes and Helicopters; Academic Press, New York, 1964.

26. Sherman, W.I., "A Theoretical Analysis of Airplane Longitudinal Stability and Control as Affected by Wind Shear", NASA TN-D-8496, July 1979.

27. Erzberger; H., "Analysis and Design of Model Following Control systems by state Space Techniques", Proceedings of the 1968 Joint Automatic Control Conference, June 1968 .

28. Nichols, R., and Malagon, M., "VRA and ARA Calibration Data Manual", MAE-1521, Princeton University, July 1981 .

29. Anon.; "Approval of Area Navigation Systems for Use in the U.S. National Airspace System", FAA Advisory Circular AC-90-45A, February 1975.

30. Wolowicz, C.H., "Considerations in the Determination of Stability and Control Derivatives and Dynamic Characteristics from Flight Data", AGARD Report 549, Part 1, 1966.

31. Brokof, U., and Hurrass, K., "Integrated Navigation Systems Based on Multiple DME", in AGARD-CP-298, October 1980.

32. Kayton, M., and Fried, W.R., Avionics Navigation Systems, J. Wiley and Sons, New York, 1969.

33. Newton, G.C., Gould, L.A., and Kaiser, J.F., Analytical Design of Linear Feedback Controls, J. Wiley and Sons, New York, 1957.

34. Wiener, N., The Extrapolation, Interpolation and Smoothing of Stationary Time Series, M.I.T. Press, Cambridge, 1949.

35. Kalman, R.E., and Bucy, R., "New Results in Linear Filtering and Prediction", Journal of Basic Engineering (ASME); Vol. 83D; June 1961.

36. Anderson, B.D., and Moore, J.B., Optimal Filtering, Prentice-Hall, EnglewooD Cliffs, 1979.

37. Gelb, A.; et al., Applied Optimal Estimation; M.I.T. Press, Cambridge, 1974. 
38. Wauer, J.C., "Practical Considerations in Implementing Kalman Filters", in AGARD-LS-82, May 1976.

39. Miller, G.E., "Correlation Between the Angle-of-Attack Vane Position and the Aerodynamic Angle-of-Attack of the Princeton Avionics Research Aircraft", Internal Report, Flight research Laboratory, Princeton University, July 1973.

40. Latham, R.W., and Townes, R.S., "DME Errors", Navigation: Journal of the Institute of Navigation, Vol. 22, No. 1, 1976.

41. Bryson, A.E., "Kalman Filter Divergence and Aircraft Motion estimators", AIAA Journal of Guidance and Control, Vol. 1, No. 1, January-February 1978.

42. Schmidt, S.F., "Computational Techniques in Kalman Filtering", in AGARD-ograph 139, 1970.

43. White, J.S., and Lee, H.Q., "The Variable Dimension Automatic Synthesis Program", NASA TM X-2417, October 1971.

44. Gentleman, W.M., "Least Square Computations by Givens Transformations Without Square Roots", Journal of the Institute of Mathematics Applications, Vol. 12, 1973.

45. Thornton, C.L., and Bierman, G.J., "A Numerical Comparison of Discrete Kalman Filtering Algorithm: An Orbit Determination Case Study", Automatica, January 1977.

46. Rauch, H.E., Tung, F., and Striebel, C.T., "Maximum Likelihood Estimates of Linear Dynamic Systems", AIAA Journal; Vol. 3, No. 8, August 1965.

47. Fraser, D.C., "A New Technique for the Optimal Smoothing of Data", Ph.D. Thesis, Massachusetts Institute of Technology, January 1967.

48. Fraser, D.C., and Potter, J.E., "The Optimum Linear Smoother as a Combination of Two Optimum Linear Filters", IEEE Trans. on Automatic Control, Vol.7, No. 8, August 1969.

49. Bach, R.E., "A Variational Technique for Smoothing Flight-Test and Accident Data", AIAA 80-1601, August 1980 .

50. Kwakernaak, H., and Sivan, R., Linear Optimal Control Systems, Wiley-Interscience, New York, 1972. 
51. Widnall, W.S., and Sinha, P.K., "Optimizing the Gains of the Baro-Inertial Vertical Channel", Journal of Guidance and Control, Vol. 3, No. 2, March-April 1980.

52. Widnall, W.S., and Bryson, A.E., "Comment on 'Optimizing the Gains of the Baro-Inertial Vertical Channel' ", Journal of Guidance and Control, Vol. 3, No. 3, May-June 1980.

53. Anon., "IMSL Library-Mathematical and statistical FORTRAN Subroutines", Reference Manual, IMSI LIB-0007, Houston, January 1979.

54. Papoulis, A., Probability, Random Variables and Stochastic Processes, McGraw-Hill, New York, 1965.

55. Schmidt, S.F., et al., "Application of Kalman Filtering to the C-5 Guidance and Control System", in AGARDogragh 139, 1970.

56. Bekey; G.A., and Karplus, W.J., Hybrid Computation, J. Wiley and Sons, New York, 1968.

57. Bryson, A.E., and Bobick, J.C., "Improved Navigation by Combining VOR/DME Information and Air Data", AIAA Paper 71-928, August 1971.

58. Otnes, R.K., and Enochson, L., Applied Time Series Analysis; J. Wiley and Sons, New York, 1972.

59. Draper, N.R., and Smith, H., Applied Regression Analysis, J. Wiley and Sons, New York, 1981 .

60. Chatterjee, S., and Price, B., Regression Analysis by Example, J. Wiley and Sons, New York, 1977.

61. Cooper, G.E., and Harper, R.P., "The Use of Pilot Rating in the Evaluation of Aircraft Handling Qualities", NASA TN D-5153, April 1969.

62. Sheridan, T.B.; "Mental Workload in Decision and Control", IEEE Conference on Decision and Control, 1979 .

63. Roscoe, A.H., ed., "Assessing Pilot Workload", AGARDAG-233, February 1978 .

64. Dick, A.O.: "Instrument Scanning and Controlling: Using Eye Movement Data to Understand Pilot Behavior and Strategies", NASA CR 3306, September 1980.

65. Hartman, B.0., ed., "Higher Mental Functioning in Operational Environment", AGARD-CP-181, Apri1 1976. 
66. Auffret, R., ed., "Studies on Pilot Workload", AGARDCP-217, November 1977.

67. Barber, M.R.; "An Evaluation of the Handling Qualities of Seven General Aviation Aircraft", NASA TN D-3726, November 1966 .

68. Rosenberg, B., et al., "Critical Tracking Task Workload Rating Study", FAA-EM-81-13, September 1981 .

69. Adamson, J.C., et al., "A Simulator Study of Control and Display Augmentations for Helicopters", MAE-1428, Flight Research Laboratory, Princeton University, January 1980 .

70. Miller, G.E., and Trascos, R.L., "Flight Evaluation of Engine Response, Flight Path Stability, and Direct Lift Control", Princeton University Report No. 888, August 1971 .

71. Steinberger,J.M.; "In-Flight Simulation of the General Aviation Aircraft Stall", Princeton University, MAE-1451T, August 1979.

72. Bar-Gill, A., et al., "Development of Flying Qualities Criteria for Single Pilot Instrument Flight operations: Interim Report", NASA CR 165932, June 1982.

73. Anon., Jeppesen Airway Manual; Jeppesen and Co., Denver, 1981. 


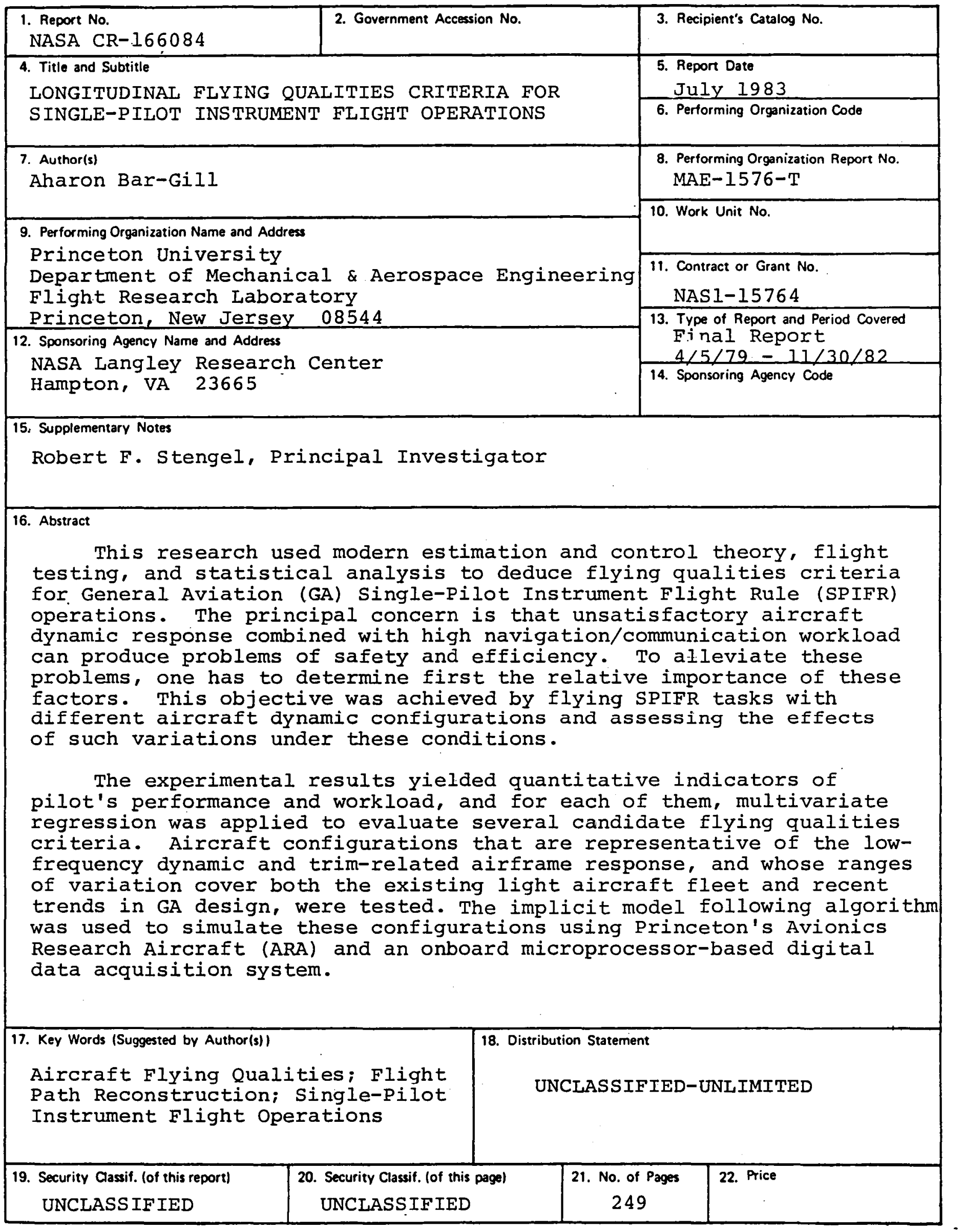




\section{End of Document}

